FLÁVIO DE OLIVEIRA SILVA

\title{
ENDEREÇAMENTO POR TÍTULO: UMA FORMA DE ENCAMINHAMENTO MULTICAST PARA A PRÓXIMA GERAÇÃO DE REDES DE COMPUTADORES
}


FLÁVIO DE OLIVEIRA SILVA

\section{ENDEREÇAMENTO POR TÍTULO: UMA FORMA DE ENCAMINHAMENTO MULTICAST PARA A PRÓXIMA GERAÇÃO DE REDES DE COMPUTADORES}

Tese apresentada à Escola Politécnica da Universidade de São Paulo para obtenção do Título de Doutor em Ciências. 


\section{ENDEREÇAMENTO POR TÍTULO: UMA FORMA DE ENCAMINHAMENTO MULTICAST PARA A PRÓXIMA GERAÇÃO DE REDES DE COMPUTADORES}

Tese apresentada à Escola Politécnica da Universidade de São Paulo para obtenção do Título de Doutor em Ciências.

Área de Concentração:

Sistemas Eletrônicos

Orientador:

Prof. Dr. Sergio Takeo Kofuji

Co-orientador:

Prof. Dr. Pedro Frosi Rosa 
Este exemplar foi revisado e alterado em relação à versão original, sob responsabilidade única do autor e com a anuência de seu orientador.

São Paulo, 03 de dezembro de 2013.

Assinatura do autor

Assinatura do orientador

Silva, Flávio de Oliveira

Endereçamento por título: uma forma de encaminhamento multicast para a próxima geração de redes de computadores I F.O. Silva. -- versão corr. -- São Paulo, 2013. $184 \mathrm{p}$.

Tese (Doutorado) - Escola Politécnica da Universidade de São Paulo. Departamento de Engenharia de Sistemas Eletrônicos.

1.Redes e comunicação de dados 2.Protocolos de comunicação 3.Internet 4.Engenharia de sistemas de computação 5.Redes de computadores I.Universidade de São Paulo. Escola Politécnica. Departamento de Engenharia de Sistemas Eletrônicos II.t. 
Dedico este trabalho à memória daqueles que estão vivos em nossos corações. Meu Pai, Geraldo Antônio da Silva, que viveu sua vida pela minha, e a João Roberto Horta, um grande incentivador. 


\section{AGRADECIMENTOS}

Agradeço a Deus pelo dom da vida, de graça recebido. A Ele também agradeço, pela família que me concedeu e por colocar em minha vida, pessoas especiais.

Agradeço à minha família: à Andréa, pelo amor, suporte e dedicação. Agradeço pela Elyse e pelo Elyas, presentes de Deus, frutos do nosso amor, que representam o nosso futuro e que nos revigoram com a sua alegria, alimentando a criança que existe em nós. À minha mãe, que sempre, silenciosamente, velou por mim. À Mariana, uma antítese do estigma da sogra.

Mais importante que resultados científicos, são os novos amigos construídos ao longo deste trabalho. Por eles também é preciso agradecer.

Agradeço ao Sérgio Takeo, pela contribuição na minha formação. Ao final deste trabalho perco um orientador, mas ganho um amigo sincero e com grande coração, que comigo continuará nesta aventura científica.

Agradeço aos colegas do PAD, representados pelo Elvis, Kenji e Stelvio, pelo apoio, pela solicitude sempre dispensada e pelas novas parcerias.

Aos colegas do programa MEHAR (Mondial Entities Horizontally Addressed by Requirements): Alex Dias, Caio Ferreira, Edmo Lopes, João Eurípedes, João Henrique, Luiz Cláudio, Maurício Gonçalves e Natal Neto. Apoiadores de primeira hora, companheiros na jornada. Que possamos continuar juntos em novos projetos e desafios.

Agradeço ao Augusto Neto, mais que um apoiador deste trabalho e parceiro na pesquisa, um amigo. Agradeço aos amigos construídos além-mar: Carlos Guimarães, Daniel Corujo e Rui Aguiar.

Agradeço ao Lásaro e ao Pasquini, colegas da FACOM, pela colaboração e compartilhamento de ideias durante o desenvolvimento deste trabalho.

Agradeço ao Pedro Frosi, amigo íntegro e valoroso, parceiro de trabalho, que não busca seus próprios interesses e com quem tenho a graça conviver.

Agradeço aos docentes da Faculdade de Computação, colegas de trabaIho que apoiaram das mais diversas formas: Anilton, Daniel, Denise, Elaine, Fabiano, Faina, Ilmério, Jamil, João Nunes e Mauricio.

Agradeço a outros que acreditaram e apoiaram este trabalho: O Programa Santander de Bolsas de Mobilidade Internacional, à Comissão Europeia pelo financiamento através do projeto OFELIA, à Escola Politécnica da USP e à Universidade Federal de Uberlândia.

Somos parte de um todo e sozinhos nada realizamos, portanto, agradeço a todos sem distinção. 
Recomenda a Yahweh tuas obras, e teus projetos se realizarão. $\operatorname{Pr} 16,3$ 


\section{RESUMO}

A Internet foi projetada em contexto distante da realidade atual. Novas aplicações, novas tecnologias e dispositivos definem um novo conjunto de requisitos, que, entre outros, consiste de suporte a mobilidade, segurança, QoS e multicast. Pesquisadores de todo o mundo estão engajados no projeto da Internet do Futuro. Enquanto muitos procuram evoluir a Internet atual, outros partem do princípio de que arquitetura atual deve ser ignorada a fim de se conceber uma nova arquitetura. Partindo de uma visão disruptiva, este trabalho tem por objetivo definir, projetar e avaliar experimentalmente uma forma de encaminhamento onde o multicast seja um requisito central para as aplicações. Essa forma de encaminhamento é baseada em novas relações entre entidades que se comunicam e na utilização de uma identificação/endereçamento única, não ambígua e independente da topologia: seu Título. A arquitetura Entity Title Architecture (ETArch), construída sobre uma infraestrutura baseada em redes definidas por software, estabelece novas relações entre as entidades de sistemas distribuídos. A definição do Workspace, implementado neste trabalho, permite estabelecer um canal por onde múltiplas entidades se comunicam naturalmente de uma forma multicast, permitindo ainda a mobilidade dessas entidades ao longo da rede. Identificado por seu Título, o Workspace é o destino das primitivas durante a comunicação. À medida que novas entidades se ligam ou desligam, há reconfiguração do Workspace. A abordagem definida, implementada e verificada neste trabalho representa uma forma mais eficiente de comunicação se comparada aos endereços no âmbito da camada de rede (IP Multicast) ou de aplicação (Application Layer Multicast). A arquitetura ETArch leva em conta não somente os aspectos de multicast e mobilidade das entidades, como também foi construída com a visão de incorporar em um momento futuro requisitos de QoS e segurança, sendo, portanto, um ponto de convergência para o diálogo com outros grupos de pesquisa, a fim de colaborar na construção da próxima geração das redes de computadores.

Palavras Chave: Redes de Computadores. Arquitetura de Rede. Título de Entidade. Multicast. Mobilidade. Redes Definidas por Software. 


\section{ABSTRACT}

Internet was designed in a totally different context, far from what we have today. New applications, new technologies and devices define a new set of requirements, which among others, is to support mobility, security, QoS and multicast. Researchers around the world are engaged in the design of the Future Internet. While many seek to evolve the current Internet, others assume that current architecture should be ignored in order to design a new architecture. From a disruptive vision, this work aims to define, design and experimentally validate a form of referral where multicast be a central requirement for applications. This form of routing is based on new relationships between entities that communicate and use of an identification/addressing based on a topology independent name that unambiguously identifies an entity: its Title. The Entity Title Architecture (ETArch), built on an infrastructure based on software defined networking, establishes new relationships between entities in distributed systems. The definition of the Workspace, implemented in this work, allows establishing a channel through which multiple entities communicate naturally by using a multicast approach, while still allowing mobility of these entities over network. Identified by its title, the Workspace is the destination of the primitives during communication. As new entities attach or detach the Workspace is reconfigured. The approach defined, implemented and verified in this work is a more efficient way of communication compared to the addressing within the network layer (IP Multicast) or application (Application Layer Multicast). ETArch takes into account not only the aspects of multicast and mobility of the entities, but also was built with the vision to incorporate into a future time QoS and security, so it is a focal point for dialogue with other research groups to collaborate in building the next generation of computer networks.

Keywords: Computer Networks. Network Architecture. Entity Title. Multicast. Mobility. Software Defined Networking. 


\section{LISTA DE ILUSTRAÇÕES}

1 Crescimento Tráfego Originado por Dispositivos Móveis $(\mathrm{CISCO}, 2013) \ldots \ldots \ldots \ldots \ldots \ldots \ldots$

2 Arquitetura dos Primeiros Computadores. . . . . . . . . . . . . . 40

3 Endereçamentos: (a) Unicast (b) Multicast (c) Broadcast. . . . . 41

4 Endereçamento MAC IEEE 802.3. . . . . . . . . . . . . . . . . . 42

5 Membros do Grupo Multicast e Árvore de Cobertura com Raiz na Origem. . . . . . . . . . . . . . . . . . 46

6 Visão Geral de Intelligent Network. . . . . . . . . . . . . . . . . . 52

$7 \quad$ Elementos Físicos de Intelligent Network . . . . . . . . . . . 55

8 Diagrama da Infraestrutura FORCES (YANG et al., 2004). . . . . 56

$9 \quad$ Interface Forces: funções CE e FE. . . . . . . . . . . . . . . 57

10 Componentes de um OpenFlow Switch (PFAFF et al., 2011). . . 59

11 Componentes do Modelo MIH (IEEE, 2009). . . . . . . . . . . . 63

12 Processo para definição da ETArch (Entity Title Archicture) (BASS; CLEMENTS; KAZMAN, 2003). . . . . . . . . . . . . . 76

13 Principais Componentes da Arquitetura - DTS, DTSA, Entity, Title e o Workspace. . . . . . . . . . . . . . . . . . 83

14 Workspaces Públicos e Privados no DTS. . . . . . . . . . . . . . 84

15 Workspace de Dados. . . . . . . . . . . . . . . . . 86

16 Visão Geral do DTS . . . . . . . . . . . . . . . . . . . . . 87 
17 DTS como uma Rede Mundial em Três Níveis. . . . . . . . . . . 90

18 Componentes de um Nível Genérico do DTS. . . . . . . . . . . . 91

19 MDTSA - Exemplos de valores no Workspace Database e DTS Resolver Database. . . . . . . . . . . . . . . . . . . . . . . . . . 92

20 Camadas definidas pela ETArch. . . . . . . . . . . . . . . . . . . 94

21 Identificação e Endereçamento. . . . . . . . . . . . . . . . . . . 99

22 ETArch Protocols. . . . . . . . . . . . . . . . . . 101

23 Primitivas ECTP e OpenFlow Trocadas a Fim de Criar e Adaptar o Workspace.

24 Componentes da Arquitetura do DTSA Implantados no JAIN

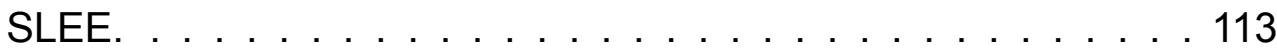

25 Cenário Utilizado para Experimentação. . . . . . . . . . . . . . . 115

26 Comparação no uso da Banda de Dados entre Arquiteturas TCP/IP e ETArch.

27 Inicialização do Cenário utilizando protocolo ETCP . . . . . . . . 119

28 Handover Assistido pelo Mobile Node e Controlado pela Rede, entre Dois Diferentes DTSAs . . . . . . . . . . . . . . . . . . 120

29 Cenário de Avaliação do Handover de uma entidade (MN). . . . 122

30 Cena do Vídeo durante Handover de Entidade (MN2) anexada ao Workspace.

31 Tela com Tráfego das Interfaces de Rede do MN2 durante Handover. . . . . . . . . . . . . . . . . . . 124 
32 Recepção de Conteúdo no MN2 ao Longo do Tempo de Experimento. . . . . . . . . . . . . . . . . . . . . . 125

33 Formato Geral da Primitiva dos Protocolos ETCP e DTSCP. . . . 158

34 Diagrama de Sequência do Serviço ENTITY-REGISTER . . . . 159

35 Diagrama de Sequência do Serviço WORKSPACE-CREATE. . . 161

36 Diagrama de Sequência do Serviço WORKSPACE-ATTACH. . . 164

37 Diagrama de Sequência do Serviço ENTITY-UNREGISTER. . . 167

38 Diagrama de Sequência do Serviço WORKSPACE-DETACH. . . 169

39 Diagrama de Sequência do Serviço WORKSPACE-DELETE. . . 171

40 Diagrama de Sequência do Serviço DTSA-REGISTER. . . . . . 174

41 Diagrama de Sequência do Serviço DTS-MESSAGE. . . . . . . 176

42 Diagrama de Sequência do Serviço WORKSPACE-ADVERTISE. 179

43 Diagrama de Sequência do Serviço WORKSPACE-LOOKUP. . . 182 


\section{LISTA DE TABELAS}

1 Comparação entre as Versões do OpenFlow. . . . . . . . . . . . 61

2 Quadro Comparativo Entre as Tecnologias de Enlaces . . . . . . 65

3 Comparação entre Arquiteturas de Internet do Futuro XIA e MobilityFirst. . . . . . . . . . . . . . . . . 74

4 Primitivas do Entity Title Control Protocol(ETCP). . . . . . . . . . 102

$5 \quad$ ETCP Application Programming Interface. . . . . . . . . . . . . . 102

6 Primitivas do DTS Control Protocol(DTSCP). . . . . . . . . . . 103

7 Primitivas do ETCP e sua Relação com o OpenFlow. . . . . . . . . 111

8 Impacto do Handover sobre a Transmissão do Conteúdo Através do Workspace. . . . . . . . . . . . . . . . . . . . . . . . 126

9 Sinalização de Controle de Cada Protocolo Envolvido. . . . . . . . 127

10 Comparação entre Arquiteturas de Internet do Futuro . . . . . 129

11 Tipos de Dados Utilizados nos Protocolos ETCP e DTSCP. . . . 152

12 Produção Bibliográfica Resultante Deste Trabalho. . . . . . . . . 154

13 Semântica dos Campos das Primitivas de Controle . . . . . . . . 158

14 Semântica da Primitiva de Serviço ENTITY-REGISTER.req. . . . 160

15 Semântica da Primitiva de Serviço WORKSPACE-CREATE.req. 163

16 Semântica da Primitiva de Serviço WORKSPACE-ATTACH.req. 166

17 Semântica da Primitiva de Serviço ENTITY-UNREGISTER.req. . 168 
18 Semântica da Primitiva de Serviço WORKSPACE-DETACH.req. 170

19 Semântica da Primitiva de Serviço WORKSPACE-DELETE.req. 173

20 Semântica da Primitiva de Serviço DTSA-REGISTER.req. . . . . 175

21 Semântica da Primitiva de Serviço DTS-MESSAGE.req. . . . . . 178

22 Semântica da Primitiva de Serviço WORKSPACE-ADVERTISE.req. . . . . . . . . . . . . . . . . 181

23 Semântica da Primitiva de Serviço WORKSPACE-LOOKUP.req. 183

24 Semântica da Primitiva de Serviço WORKSPACE-LOOKUP.resp. 184 


\section{LISTA DE ABREVIATURAS E SIGLAS}

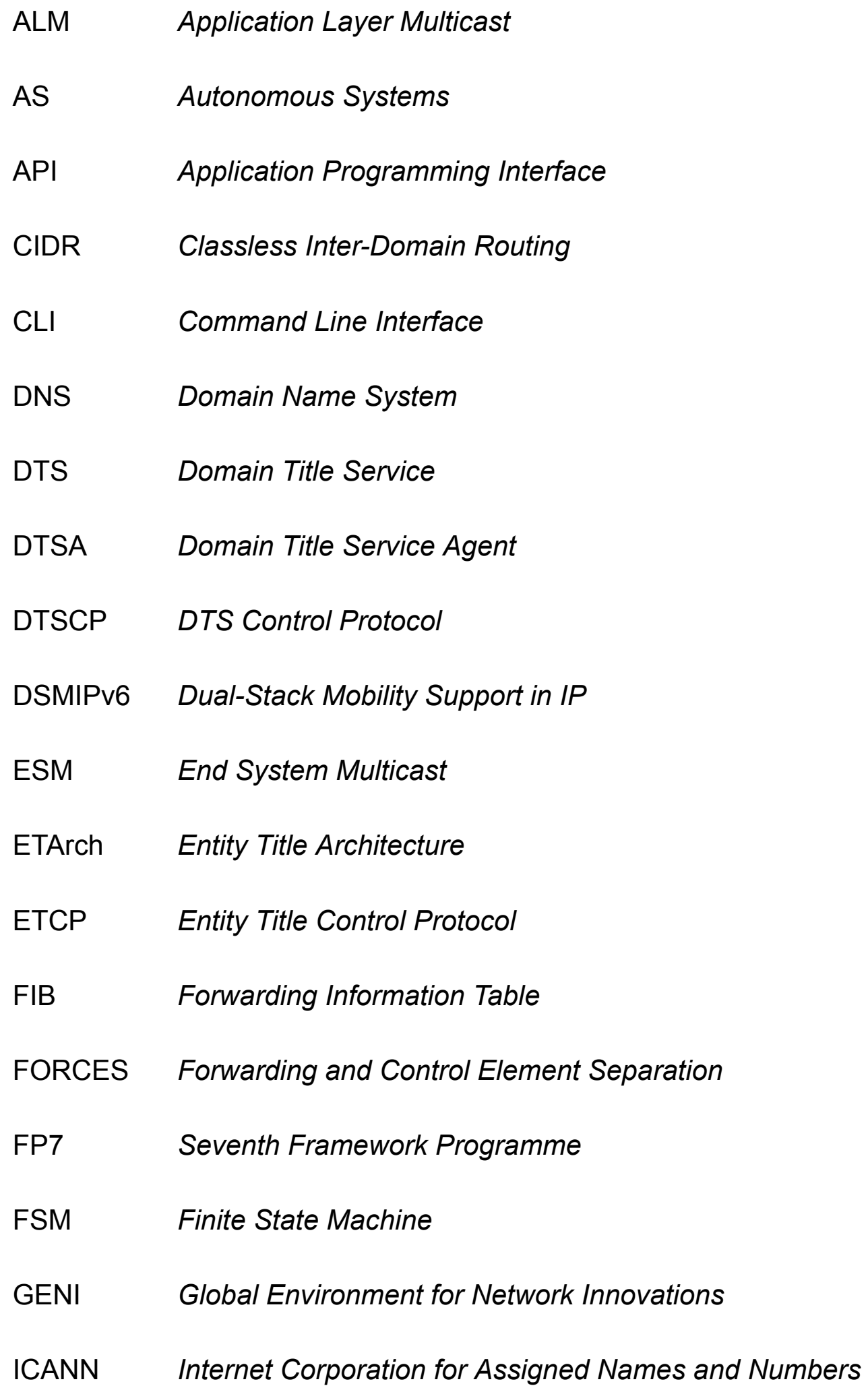




$\begin{array}{ll}\text { ICMPv6 } & \text { Internet Control Message Protocol version } 6 \\ \text { IETF } & \text { Internet Engineering Task Force } \\ \text { IP } & \text { Internet Protocol } \\ \text { IPv4 } & \text { Internet Protocol version } 4 \\ \text { IPv6 } & \text { Internet Protocol version } 6 \\ \text { ISP } & \text { Internet Service Provider } \\ \text { MDTSA } & \text { Master DTSA } \\ \text { MIP } & \text { Mobile IP } \\ \text { MIPv6 } & \text { Mobile IPv6 } \\ \text { MIHF } & \text { Multimedia Independent Handover Function } \\ \text { NAT } & \text { Network Address Translation } \\ \text { NE } & \text { Network Element } \\ \text { NLR } & \text { National Lambda Rail } \\ \text { NSF } & \text { National Science Foundation } \\ \text { OSI } & \text { Open Systems Interconnection } \\ \text { PDU } & \text { Protocol Data Unit } \\ \text { PoA } & \text { Point of Attachment } \\ \text { PoS } & \text { Point of Service } \\ \text { QoE } & \text { Quality of Experience } \\ \text { SDN } & \text { Quality of Service } \\ \text { Service Building Block } \\ \text { Software Defined Networking }\end{array}$




$\begin{array}{ll}\text { RA } & \text { Resource Adaptor } \\ \text { RIB } & \text { Routing Information Base } \\ \text { RTP } & \text { Real-time Transport Protocol } \\ \text { UDP } & \text { User Datagram Protocol }\end{array}$




\section{SUMÁRIO}

1 Introdução 22

1.1 Motivação . . . . . . . . . . . . . . . . . . . . . . . 28

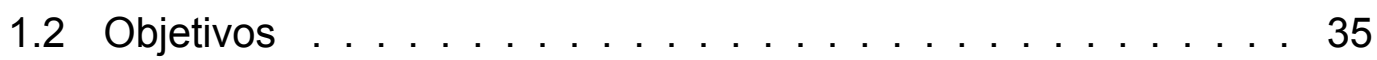

1.3 Organização . . . . . . . . . . . . . . . . 35

2 Revisão Bibliográfica $\quad 38$

2.1 Aspectos de Endereçamento em Redes de Computadores . . . 39

2.1.1 Aspectos Conceituais e Filosóficos de Endereçamento . 39

2.1.2 Endereçamento Multicast na Camada de Rede . . . . . . 43

2.1.3 Endereçamento Multicast na Camada de Aplicação . . . 46

2.2 Ortogonalidade entre Planos de Controle e de Dados . . . . . . 49

2.2.1 Redes Inteligentes em Telecomunicações . . . . . . . . . 51

2.2.2 Visão Geral da Infraestrutura FORCES . . . . . . . . . . 55

2.2.3 Redes Definidas por Software . . . . . . . . . . . . 57

2.2.3.1 Especificações do OpenFlow . . . . . . . . . . . 60

2.2.3.2 Media Independent Handover Services . . . . . 62

2.2.3.3 Comparação entre Tecnologias de Enlaces . . . 64

2.3 Pesquisa Experimental em Arquiteturas para a Internet do Futuro 66

2.3.1 Instalações Experimentais . . . . . . . . . . . 66 
2.3.2 Novas Arquiteturas para a Internet do Futuro . . . . . . . 68

2.3.2.1 MobilityFirst . . . . . . . . . . . . 69

2.3.2.2 eXpressive Internet Architecture . . . . . . . 71

2.3.3 Análise Comparativa . . . . . . . . . . . . . . . . . . . 72

3 Especificação da Arquitetura ETArch $\quad 75$

3.1 Aspectos Conceituais da ETArch . . . . . . . . . . . . . . . 77

3.1 .1 Entidade . . . . . . . . . . . . . . . 77

3.1 .2 Título . . . . . . . . . . . . . . . 78

3.1 .3 Workspace . . . . . . . . . . . . . . . 80

3.1.3.1 Workspace de Controle . . . . . . . . . . . 83

3.1.3.2 Workspaces de Dados . . . . . . . . . . . 85

3.1.4 Serviço de Domínio de Títulos . . . . . . . . . . . . . 86

3.2 Camadas ETArch . . . . . . . . . . . . . . . . . 93

3.3 Concepção de Serviços na ETArch . . . . . . . . . . . . . . . . 96

3.4 Identificação e Endereçamento . . . . . . . . . . . . . . . . . 97

3.5 Protocolos ETArch . . . . . . . . . . . . . . . . 100

3.5.1 Entity Title Control Protocol . . . . . . . . . . . . . . . . . 101

3.5 .2 DTS Control Protocol . . . . . . . . . . . . . 102

3.6 Aspectos da Rede . . . . . . . . . . . . . . . . . . . 103

3.6.1 Roteamento . . . . . . . . . . . . . . . . 104

3.6.2 Mobilidade . . . . . . . . . . . . . 105 
4 Arquitetura ETArch: Implementação e Análise

4.1 Aspectos de Implementação . . . . . . . . . . . . . . . . 109

4.2 Avaliação Experimental . . . . . . . . . . . . . . . . . 114

4.2.1 Comunicação Baseada no Workspace . . . . . . . . . . 114

4.2.2 Mobilidade Através do Workspace . . . . . . . . . . . 118

4.2.3 Handover de Entidades . . . . . . . . . . . . . . . . . 121

4.3 Análise de Resultados . . . . . . . . . . . . . . . . . . . 128

5 Conclusão e Trabalhos Futuros 133

$\begin{array}{ll}\text { Referências } & 138\end{array}$

Apêndice A - Definição de Tipos de Dados 152

Apêndice B - Resultados $\quad 154$

Apêndice C - Protocolos da Arquitetura ETArch 157

C.1 Formato das Primitivas . . . . . . . . . . . . . . . 157

C.2 Entity Title Control Protocol . . . . . . . . . . . . . . . 159

C.2.1 ENTITY-REGISTER . . . . . . . . . . . . . . . . . . 159

C.2.1.1 ENTITY-REGISTER.req . . . . . . . . . . . . 160

C.2.1.2 ENTITY-REGISTER.resp . . . . . . . . . . . 161

C.2.2 WORKSPACE-CREATE . . . . . . . . . . . . . . 161

C.2.2.1 WORKSPACE-CREATE.req . . . . . . . . 162

C.2.2.2 WORKSPACE-CREATE.resp . . . . . . . . . 162 
C.2.3 WORKSPACE-ATTACH . . . . . . . . . . . . . 164

C.2.3.1 WORKSPACE-ATTACH.req . . . . . . 165

C.2.3.2 WORKSPACE-ATTACH.resp . . . . . . . . 166

C.2.4 ENTITY-UNREGISTER . . . . . . . . . . . . . 166

C.2.4.1 ENTITY-UNREGISTER.req . . . . . . . . . . 167

C.2.4.2 ENTITY-UNREGISTER.resp . . . . . . . . . 168

C.2.5 WORKSPACE-DETACH . . . . . . . . . . . 168

C.2.5.1 WORKSPACE-DETACH.req . . . . . . . 170

C.2.5.2 WORKSPACE-DETACH.resp . . . . . . 170

C.2.6 WORKSPACE-DELETE . . . . . . . . . . . . . 171

C.2.6.1 WORKSPACE-DELETE.req . . . . . . . . 172

C.2.6.2 WORKSPACE-DELETE.resp . . . . . . . . . 173

C.3 DTS Control Protocol . . . . . . . . . . . . . . . . . 173

C.3.1 DTSA-REGISTER . . . . . . . . . . . . . . . 173

C.3.1.1 DTSA-REGISTER.req . . . . . . . . . . . . . 175

C.3.1.2 DTSA-REGISTER.resp . . . . . . . . . . . . 175

C.3.2 DTS-MESSAGE . . . . . . . . . . . . . . 176

C.3.2.1 DTS-MESSAGE.req . . . . . . . . . . . . 177

C.3.2.2 DTS-MESSAGE.resp . . . . . . . . . . . 177

C.3.3 WORKSPACE-ADVERTISE . . . . . . . . . . . 178

C.3.3.1 WORKSPACE-ADVERTISE.req . . . . . . 180

C.3.3.2 WORKSPACE-ADVERTISE.resp . . . . . 180 
C.3.4 WORKSPACE-LOOKUP . . . . . . . . . . . . . . 181

C.3.4.1 WORKSPACE-LOOKUP.req . . . . . . . . 183

C.3.4.2 WORKSPACE-LOOKUP.resp . . . . . . . . 184 


\section{INTRODUÇÃO}

A concepção da Internet remonta à década de sessenta (BARAN, 1964) e os seus principais protocolos foram propostos no início dos anos setenta (CERF; KAHN, 1974). Após quatro décadas, a Internet provocou um extraordinário impacto na sociedade.

Esse impacto foi potencializado pela evolução tecnológica nesse período, que foi decisiva para o contexto atual. O desenvolvimento do hardware, conforme preconizado pela Lei de Moore, permitiu a criação de dispositivos com uma enorme capacidade de processamento quando comparado com os recursos dos equipamentos que inicialmente utilizaram a rede. Além disso, ocorreu a ampliação da capacidade de vazão de enlaces e diminuição de preços que permitem enorme capilaridade.

Esse conjunto de tecnologias e evoluções permitiram a criação de aplicações e serviços que atualmente fazem parte do cotidiano de pessoas nos campos profissional e pessoal, alterando suas vidas em diferentes aspectos.

Neste contexto, as novas aplicações alteram completamente o padrão de tráfego das redes de computadores e, portanto, da Internet. A popularização de aplicações multimídia (ensino à distância, vídeo conferência, internet TV, jogos on line, voz sobre IP, etc.), por exemplo, é notória. Previsões feitas pela Cisco (CISCO, 2013) destacam que até 2017, esse tipo de tráfego ocupará $73 \%$ do volume total da Internet, com crescimento anual de $27 \%$ a partir de 
2012. As mesmas previsões indicam que o tráfego na Internet, oriundo de dispositivos móveis (colunas Mobile Data e Fixed/Wi-Fi representadas na Figura 1), corresponderá a $68.3 \%$ do tráfego IP global, mostrando clara tendência de aplicações multimídia em cenários onde os consumidores, e também produtores, residem em dispositivos móveis.

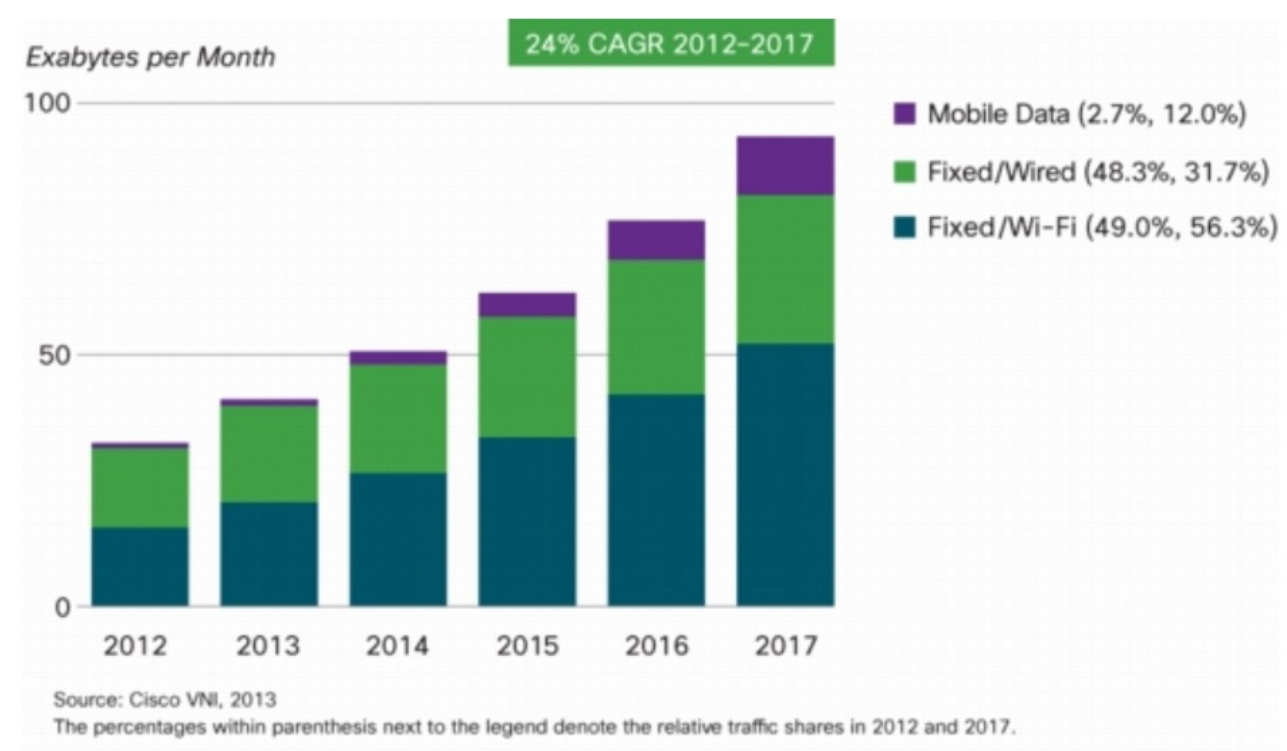

Figura 1: Crescimento Tráfego Originado por Dispositivos Móveis (CISCO, 2013).

De acordo com o IDC (IDC, 2013), em 2012, mais de um bilhão de dispositivos classificados como smart connected devices (classificação que engloba computadores pessoais portáteis e não portáteis, smartphones e tablets) foram comercializados pelos fabricantes. Segundo o mesmo estudo, em 2017 os smartphones corresponderão a $67 \%$ desse mercado, enquanto computadores pessoais não portáteis corresponderão a $6 \%$. Além de possuir um hardware poderoso, normalmente com mais de um câmera de vídeo e diversos sensores, os smartphones possuem interfaces para diferentes tecnologias de redes sem fio, levando a um cenário que engloba não apenas a mobilidade de localização, mas também a mobilidade entre tecnologias de redes de acesso.

Todas essas tendências de dispositivos e novas aplicações impõem sobre 
as redes, incluindo a Internet, um conjunto de requisitos que sequer podiam ser imaginados durante sua criação há quatro décadas. Em suma, os requisitos atuais compreendem aspectos como: mobilidade; segurança; qualidade de serviço (QoS); suporte mais eficiente a aplicações peer to peer; sensores; dispositivos móveis; conteúdos produzidos em tempo real como voz e vídeo de alta qualidade com fontes móveis, etc. Outra necessidade muito importante está relacionada ao gerenciamento de rede, cuja complexidade aumenta a cada dia, à medida que novos protocolos de controle (EDER; NAG, 2001) e appliances são implantados na rede. Além de aspectos técnicos, os requisitos desses novos cenários compreendem questões tão distintas como eficiência energética, segurança e necessidades sociais e econômicas (TSELENTIS et al., 2009).

Entretanto, após extraordinário impacto na sociedade, a Internet enfrenta grandes desafios para sua evolução (ZAHARIADIS et al., 2011). A necessidade de mudança na arquitetura é algo que a comunidade científica vislumbra desde o início da década de noventa (CLARK et al., 1991). Desde sua proposição inicial, a arquitetura passou por várias evoluções em aspectos como, por exemplo, endereçamento (EGEVANG; FRANCIS, 1994) (SRISURESH; EGEVANG, 2001) (GROUP; HINDEN, 1993a) e roteamento (REKHTER; LI, 1995). Um fator que normalmente motivou e impulsionou essas evoluções foi a necessidade de adaptar-se às novas escalas da Internet à medida que seu crescimento exponencial acontecia, ampliando consideravelmente a complexidade da arquitetura, especialmente no plano de controle (AGUIAR, 2008). Certas mudanças, porém, decorrentes de novos requisitos na atual arquitetura, são de difícil implantação (GHODSI et al., 2011).

Com essa perspectiva, pesquisadores de todo o mundo estão engajados no projeto da arquitetura da Internet do Futuro. Duas abordagens (REXFORD; 
DOVROLIS, 2010) são possíveis: evolucionária, onde a nova arquitetura deve evoluir a partir da atual; e clean slate na qual, em princípio, os pesquisadores ignoram a arquitetura legada, ficando livres para inovar e propor novas bases para uma nova arquitetura (FELDMANN, 2007)(ROBERTS, 2009).

A abordagem evolucionária sofre com a necessidade de manter compatibilidade com o legado. Um exemplo claro é o protocolo IP cuja versão IPv6 (DEERING; HINDEN, 1995), proposta inicialmente em 1995, i.e., no milênio passado, ainda não foi completamente implantada até os dias atuais (DHAMDHERE et al., 2012) (WOOD, 2011). Dados do final de julho de 2013 (NCC, 2013) do RIPE NCC indicam que no nível de AS (Autonomous Systems), $16.59 \%$ deles anunciam prefixos IPv6. Por outro lado, na abordagem clean slate, em um momento futuro, quando os resultados indicarem que devam ser implantados, a pesquisa será então reorientada para a transição entre a Internet atual e a Internet do Futuro.

Nos Estados Unidos, várias iniciativas focam em uma nova arquitetura para Internet. Uma das primeiras ações neste sentido foi o projeto NewArch (2000 a 2003) (DARPA, 2003), que vislumbrava discutir os objetivos e a direção de uma pesquisa relacionada com a próxima geração da arquitetura da Internet. Iniciado em 2005, o programa FIND (Future Internet Design) (NSF, 2009) que objetivava projetar a internet com uma visão clean slate, apoiou mais de cinquenta projetos durante cinco anos. Na sequência o programa NSF FIA (Future Internet Architecture) (FOUNDATION, 2010) contemplou cinco projetos que visam projetar e avaliar novas arquiteturas utilizando uma abordagem inovadora. Essas iniciativas são alguns exemplos de esforços de pesquisa com foco em uma nova arquitetura para a Internet. Outro exemplo é o projeto Clean Slate (STANFORD, 2006), da Universidade de Stanford, inciado em 2006 e concluído em 2012 com a missão de reinventar a 
Internet.

Patrocinado pela National Science Foundation (NSF), a iniciativa Global Environment for Network Innovations (GENI) (GENI, 2013), cuja concepção iniciou em 2005, é um laboratório virtual que permite a experimentação em escala e visa criar novas possibilidades que resultem em redes futuras como a Internet. A infraestrutura da rede GENI está distribuída pelos Estados Unidos e cada ponto de presença possui recursos (rede, computação e/ou armazenamento) que são compartilhados por pesquisadores que a utilizam. O programa GENI abriga diferentes projetos e seu desenvolvimento é baseado em um modelo espiral, onde cada ciclo dura doze meses. Atualmente o programa encontra-se na espiral cinco, iniciada em outubro de 2012.

Na Europa, o FP7 (7th Framework Programme) é o principal instrumento de financiamento utilizado pela União Europeia para apoiar atividades de pesquisa nessa área. O programa foi iniciado em 2007 e possui duração de sete anos (2007 a 2013). Nesse programa, o tema Information and Communication Technologies (ICT) possui oito Desafios (Challenges) sendo que um deles (COMMISSION, 2012b) é chamado de Pervasive and Trusted Network and Service Infrastructures.

Esse Desafio é por sua vez dividido em Objetivos. Dos seis Objetivos, dois estão ligados à pesquisa científica que engloba as novas arquiteturas de rede: 1.1 - Future Networks (COMMISSION, 2013) e 1.6 - Future Internet Research and Experimentation (FIRE) (COMMISSION, 2012a). As Chamadas (Calls) no Challenge 1 envolveram financiamento de 1,7 bilhão de euros, sendo que desses, 550 milhões de euros foram para projetos ligados ao Objetivo 1.1 e 135 milhões para projetos ligados ao Objetivo 1.6. A Comissão Europeia entende que o investimento nessa área é prioritário, a fim de sustentar a competitividade da Europa no mercado global, buscando atuar em papel de liderança 
nesse tema.

Ainda na Europa, cerca de 150 projetos ligados ao Challenge 1, criaram uma colaboração chamada Future Internet Assembly (FIA) (EURESCOM, 2012) cujo objetivo é fomentar a interação entre os diversos projetos e grupos de pesquisa a fim de criar um conceito comum ligado à Internet do Futuro. Para isto o FIA é constituído de grupos de trabalho com focos específicos como, por exemplo, Arquitetura. Além disso essa comunidade organiza eventos e publicações ligadas ao tema.

No Brasil, o projeto Future Internet Testbeds Experimentation Between Brazil and Europe (FIBRE) (FIBRE, 2013a) congrega diferentes instituições de ensino, a Rede Nacional de Pequisa (RNP) (RNP, 2011) e o Centro de Pequisa e Desenvolvimento em Telecomunicações (CPqD) (CPQD, 2013). O projeto é fruto de uma chamada conjunta entre Brasil e União Europeia e além dos participantes do Brasil existe um grupo instituições ligadas à comunidade europeia que, nesse caso, são financiados pelo FP7. O principal objetivo do projeto é criar uma infraestrutura no Brasil que visa pequisa e experimentação para a Internet do Futuro, ou seja, está alinhado com o objetivo FIRE do FP7. Essa infraestrutura interligará as instituições brasileiras e europeias, criando uma infraestrutura transcontinental. O projeto também prevê a realização de alguns casos de uso para demonstrar as possibilidades da infraestrutura construída.

O esforço da comunidade cientifica internacional, com forte apoio de governos, indica a relevância do tema, cuja busca é pela arquitetura ou por arquiteturas de redes futuras. 


\subsection{Motivação}

Considerando os novos requisitos, pode-se afirmar que a arquitetura, não apenas da Internet, mas das redes de computadores como um todo, precisa ser revista. Por outro lado, levando-se em consideração o esforço necessário para evoluir a arquitetura atual, pode-se afirmar que a abordagem capaz de florescer a Internet do Futuro é a Clean Slate.

Partindo deste princípio, a questão a ser colocada é a seguinte: por onde começa o trabalho de revisão?

Argumenta-se que a resposta para esta questão passa necessariamente pela revisão do conceito de endereçamento. Para ser mais preciso, a identificação e o endereçamento.

Identificação e endereçamento são aspectos centrais em uma rede de computadores. A comunicação envolve o envio e recebimento de dados por partes que se comunicam. A identificação indica quem é essa parte, em outras palavras, a identificação designa a existência de algo e permite a sua identificação precisa. Por outro lado o endereço indica onde essa parte está localizada e através dessa localização (SALTZER, 1993) é possível construir um rota, que indica uma maneira de chegar até essa localização, sendo então a partir desse ponto possível a comunicação entre as partes.

A Internet começou como uma rede experimental, a rede DARPA (Defense Advanced Research Program Agency), depois simplesmente ARPANET, sendo que quando o IP foi proposto, o único aspecto considerado foi o endereçamento. O mapeamento entre um nome e um endereço ficou a cargo dos protocolos das camadas superiores (POSTEL, 1980b).

Na RFC 760 (POSTEL, 1980b), que inicialmente propôs o endereçamento 
IP, o endereço foi definido como quatro octetos, sendo que os primeiros oito bits representavam o número da rede e os seguintes o endereço local do host. Se isto fosse mantido a internet teria no máximo 255 diferentes redes. Pela RFC 762 (POSTEL, 1980a) é possível ter uma idéia da escala dessa rede experimental, observando os endereços que foram atribuídos às primeiras redes que compunham a Internet. O conceito de classes de endereços somente surgiu na RFC 791 (POSTEL, 1981a).

Devido ao crescimento da Internet, com o advento do esgotamento de endereços IP e a necessidade de escalabilidade no roteamento, foram implantadas soluções de "curto prazo" (EGEVANG; FRANCIS, 1994) como o Network Address Translation (NAT) (EGEVANG; FRANCIS, 1994) (SRISURESH; EGEVANG, 2001) e Classless Inter-Domain Routing (CIDR) (GROUP; HINDEN, 1993a), enquanto se aguardava por solução de "longo prazo" (EGEVANG; FRANCIS, 1994), que viria a ser o IPv6 (DEERING; HINDEN, 1995), especificado em 1995.

Como já notado anteriormente, muito pouco da solução de "longo prazo" foi implantada até o momento, enquanto as soluções de curto prazo continuam vigentes e em expansão após todo esse tempo. Essas soluções também indicam que as questões da identificação e do endereçamento não foram abordadas da maneira mais adequada, sendo necessários remendos que por sua vez provocaram efeitos colaterais como, por exemplo, do NAT em relação a alguns protocolos da camada de aplicação (HOLDREGE; SRISURESH, 2001), necessitando novos remendos e soluções específicas para esses efeitos colaterais.

A evolução da arquitetura atual da Internet parte do princípio do uso de um novo endereçamento IP, o chamado IPv6 (DEERING; HINDEN, 1998), o que corrobora a afirmação feita anteriormente, de que a revisão da arquitetura da 
Internet parte da questão relacionada ao endereçamento.

O IPv6 foi proposto inicialmente pela RFC 1883 (DEERING; HINDEN, 1995) que posteriormente foi substituída pela RFC 2460 (DEERING; HINDEN, 1998), que descreve a estrutura do protocolo. Por outro lado, a RFC 4291 (HINDEN; DEERING, 2006) descreve como é o endereçamento utilizado pelo IPv6.

Considerando desde sua proposição, em 1995, até maio de 2013, existem 381 diferentes RFCs relacionadas ao IPv6 (IPv6Now, 2013) tratando dos diversos aspectos relacionados a sua implantação. A RFC 2460, por exemplo, sofreu atualizações que estão descritas nas seguintes RFCs: 5095 (ABLEY; SAVOLA; NEVILLE-NEIL, 2007), 5722 (KRISHNAN, 2009), 5871 (ARKKO; BRADNER, 2010), 6437 (AMANTE et al., 2011), 6564 (KRISHNAN et al., 2012), 6935 (EUBANKS; CHIMENTO; WESTERLUND, 2013) e 6946 (GONT, 2013) . Por outro lado, a RFC 4291 é a mais atual de uma série composta por: RFC 1884 (HINDEN; DEERING, 1995), RFC 2373 (HINDEN; DEERING, 1998a) e RFC 3513 (HINDEN; DEERING, 2003). Além disso, até o momento, a RFC 4291, sofreu atualizações realizadas pelas RFC 5952 (KAWAMURA; KAWASHIMA, 2010) e RFC 6052 (BAO et al., 2010).

Todavia, todo o esforço para evolução da Internet através do IPv6 vem acompanhado de uma série de desafios. Trabalhos recentes indicam que uma rede puramente baseada em IPv6 ainda está sujeita a uma série de questões ligadas, por exemplo, à falta de suporte de diferentes equipamentos incluindo smartphones, sistemas operacionais e aplicações como jogos e VoIP (ARKKO; KERANEN, 2012), além de problemas com a resolução de nomes (HAZEYAMA et al., 2011). Isso indica que o problema ultrapassa a infraestrutura de rede, passa pelo sistema operacional e chega até às aplicações. 
No que diz respeito à segurança, que é um requisito central para a Internet do Futuro, a simples adoção do IPv6 não é garantia de maior segurança e menor vulnerabilidade. Resta a necessidade de compreensão de questões relacionadas à segurança no ambiente IPv6 (CAICEDO; JOSHI; TULADHAR, 2009). Por exemplo, o maior espaço de endereçamento pode ser uma poderosa arma para spammers (RAFIEE; LOWIS; MEINEL, 2012).

O IPv6 não é nem mais nem menos seguro que o IPv4 (CHOUDHARY, 2009) e tanto o IPv6 quanto protocolos associados como o Internet Control Message Protocol version 6 (ICMPv6) apresentam novos riscos que podem ser explorados (TAIB; ALI; SHAARI, 2013) necessitando, desta forma, de contramedidas adequadas (CHOUDHARY, 2009).

Mobilidade é um outro aspecto fundamental para a Internet do Futuro. Entretanto a arquitetura utilizada na ARPANET sequer previa tal necessidade (SNOEREN; BALAKRISHNAN; KAASHOEK, 2001), já que o objetivo era o acesso, através de um terminal fixo a recursos disponíveis em um outro terminal, também estacionário. Assim, a arquitetura apresenta de forma intrínseca uma dificuldade para a manutenção da conexão de um dispositivo que se movimenta entre redes distintas (CERF; CAIN, 1983).

Diante da necessidade de evolução da Internet, o Mobile IP (MIP) (PERKINS, 1996) (PERKINS, 2002), proposto inicialmente em 1996, foi a abordagem do Internet Engineering Task Force (IETF) para este requisito. Esta solução porém apresentava uma série de limitações relacionadas, por exemplo, com a carga e o atraso devidos à sinalização entre os vários elementos da rede envolvidos (SAHA et al., 2004) que impediam uma mobilidade transparente e escalável (RAHMAN et al., 2010).

O Mobile IP sofreu varias atualizações (SOLIMAN et al., 2008) (KOODLI, 2009), que por sua vez introduziam novos efeitos colaterais, por exemplo, em 
relação ao desempenho (HUNG; DUONG, 2011) e à segurança (CAICEDO; JOSHI; TULADHAR, 2009) (AURA, 2004).

Mesmo considerando as facilidades oferecidas pelo IPv6 em relação à mobilidade (PERKINS; JOHNSON, 1996), o novo endereçamento suscita novas atualizações como o Mobile IPv6 (MIPv6) (PERKINS; JOHNSON; ARKKO, 2011) e o Dual-Stack MIPv6 (DSMIPv6) (SOLIMAN, 2009) a fim de suportar a convivência entre o IPv4 e o IPv6. Ainda assim, mesmo com todo esforço para sua evolução, a mobilidade na Internet baseada no IP continua apresentando desafios (AL-SURMI; OTHMAN; ALI, 2012).

Outro requisito, igualmente importante para Internet do Futuro, é a capacidade Multicast. O conceito de Multicast envolve o envio de informação para diferentes destinos por meio de uma única transmissão, diferentemente do modo de transmissão Unicast, onde uma informação é enviada exclusivamente para um destino.

Diversos tipos de aplicações, para as quais uma mesma informação deva chegar em diferentes destinos, podem beneficiar-se desse modo de transmissão como, por exemplo: educação à distância; serviços de informações de cotações do mercado financeiro; videoconferência; entretenimento; telemedicina; entre outros.

Em um cenário onde os produtores e consumidores, que simultaneamente acessam diferentes aplicações, estão distribuídos e em movimento ao longo da rede, esse requisito da arquitetura é igualmente importante. Seu uso pode representar economia da banda de comunicação e de recursos em um cenário onde cada vez mais os usuários da rede demandam capacidade de vazão. Além disso, em um cenário onde a distribuição de conteúdo, tanto de voz quanto de vídeo de alta qualidade, caminha na direção do uso de redes puramente baseadas em pacotes, utilização eficiente da banda de comunicação é 
um aspecto não apenas desejável mas mandatório.

Inicialmente proposto por (DEERING, 1989), o IP Multicast apresenta limitações (CHU et al., 2002) tanto do ponto de vista técnico como de negócios (DIOT et al., 2000), como, por exemplo: número limitado de endereços multicast; incapacidade do gerenciamento de grupos de forma dinâmica; limitações de segurança, visto que qualquer membro do grupo multicast pode receber as informações; arquitetura complexa para implantação na Internet; e dificuldades para gerenciamento.

O IPv6 propõe uma nova abordagem para o endereçamento multicast (HINDEN; DEERING, 1998b). Na arquitetura de endereçamento definida pelo IPv6 (HINDEN; DEERING, 2006), a função dos endereços broadcast foi substituída por endereços multicast. Além disso, no IPv6 uma interface de rede pode pertencer a diferentes grupos multicast. Os endereços multicast são definidos em diferentes escopos (HINDEN; DEERING, 2006) e a arquitetura de endereçamento prevê a alocação dinâmica de endereços IP (THALER; HANDLEY; ESTRIN, 2000) (SAVOLA, 2011).

O multicast baseado no IPv6 apresenta desafios em relação à segurança (DAVIES; KRISHNAN; SAVOLA, 2007), podendo potencializar ataques. Além disso, o multicast em cenários de mobilidade, onde usuários compartilham frequências com largura de banda limitada, apresentam uma série de desafios (ROMDHANI et al., 2004), potencializados pela junção desses dois requisitos. Este é ainda um problema de pesquisa em aberto (SCHMIDT; WAEHLISCH; FAIRHURST, 2010).

Devido a essas limitações, a implantação do IP Multicast ocorre de forma lenta na rede (YIU; CHAN, 2008) e, portanto, o que proliferou de fato na Internet foi a adoção do Application Layer Multicast (ALM) (HOSSEINI et al., 2007), também conhecido como End System Multicast (ESM), onde grande 
parte das questões citadas anteriormente são tratadas no nível da camada de aplicação, facilitando sua adoção já que não implica em mudanças na arquitetura da rede.

Se por um lado ALM possui facilidades de implantação, por outro lado, uma vantagem do IP Multicast, que é a otimização do uso da banda de comunicação (LAO et al., 2005), é parcialmente perdida (no ALM), pois as árvores de distribuição construídas envolvem o envio de múltiplas cópias de pacotes (HOSSEINI et al., 2007).

Por outro lado, mesmo no contexto de ALM, questões como mobilidade apresentam vários desafios, visto que as técnicas envolvem o multicast baseado em hosts que sofrem das limitações de mobilidade impostas pelo protocolo IP.

A arquitetura da Internet não foi concebida para suportar o conceito de Multicast em uma visão fim-a-fim (HANDLEY, 2006), dificultando seu uso por aplicações, apesar de ser considerado ótimo no nível de rede.

Partindo da visão clean slate, questões como essas devem ser abordadas com uma alteração da forma de encaminhamento de pacotes utilizadas na Internet. Contudo, a alteração da forma de encaminhamento envolve aspectos e componentes centrais da arquitetura da Internet, como o endereçamento, baseado no protocolo IP.

Dessa forma, este trabalho também parte do princípio que a raiz do problema está na arquitetura da rede, onde então devem se concentrar os esforços para sua revisão à luz de novos requisitos, novas tecnologias e capacidades atualmente disponíveis. É mister notar que outras questões como Mobilidade, QoS e Segurança também estão diretamente ligadas com a revisão dessa arquitetura. 


\subsection{Objetivos}

O objetivo geral deste trabalho é definir, projetar e avaliar experimentalmente uma forma de encaminhamento de primitivas que possa ser utilizada em cenários onde o multicast seja um requisito central para as aplicações, propiciando uma utilização eficiente de recursos. Esta forma de encaminhamento será baseada em novas relações entre entidades que se comunicam e na utilização de uma identificação/endereçamento única, não ambígua e independente da topologia: seu Título.

A fim de alcançar este objetivo, este trabalho também visa:

1. Definir e projetar um novo esquema de endereçamento para as redes de computadores, que também seja capaz de identificar de forma precisa as entidades envolvidas na comunicação, com vistas a atender requisitos de multicast e mobilidade;

2. Definir os componentes e protocolos de uma arquitetura de rede que seja capaz de acomodar não apenas esta forma de encaminhamento multicast, como também outros requisitos da próxima geração das redes de computadores, como mobilidade, segurança e QoS;

3. Descrever cenários de utilização desta forma de encaminhamento, correlacionando com requisitos de mobilidade; e

4. Implementar os componentes e experimentar aspectos destes protocolos utilizando uma infraestrutura de rede definida por software.

\subsection{Organização}

O restante deste trabalho segue organizado da seguinte forma: 
- O capítulo 2 apresenta uma revisão de aspectos de endereçamento nas redes de computadores. Além disso, apresenta tecnologias utilizadas neste trabalho como o protocolo OpenFlow e o protocolo $\mathrm{MIH}$ e finalmente apresenta propostas correlatas que visam uma nova arquitetura para a Internet.

- O capítulo 3 apresenta a especificação da arquitetura Entity Title Architecture (ETArch), apresentando os conceitos, as camadas, os componentes e sua inter-relação, sua abordagem de identificação e endereçamento, descreve de forma genérica os protocolos utilizados, bem como aspectos da rede.

- O capítulo 4 descreve uma implementação para a arquitetura ETArch, detalhando os componentes de software construídos e cujo comportamento serão detalhados no Apêndice C. Além disso, apresenta uma avaliação comparativa e uma análise de resultados experimentais obtidos no decorrer deste trabalho.

- O capítulo 5 conclui este trabalho, apresentando as considerações e contribuições. O trabalho aqui apresentado não é um ponto final, mas um ponto de partida e, portanto, são indicadas algumas sugestões de trabalhos futuros, desdobramentos desta tese, a partir de exploração de lacunas em diferentes aspectos das redes de computadores.

- O apêndice $A$ descreve tipos de dados utilizados, mapeando em tipos de dados primitivos.

- O apêndice B apresenta as contribuições originais desta tese e apresenta resultados diretos e indiretos deste trabalho de pesquisa.

- O apêndice $C$ descreve e detalha as primitivas dos protocolos utilizados na arquitetura ETArch, utilizados na comunicação entre as entidades que 
constituem a rede, bem como aquelas que a utilizam. À medida que descreve as primitivas, cita os componentes necessários para o funcionamento da arquitetura. 


\section{REVISÃO BIBLIOGRÁFICA}

Apesar de todos os estudos e esforços para a evolução da Arquitetura Internet e também dos avanços nas tecnologias de comunicação desde os anos 70, não há ainda uma perspectiva clara sobre a direção a ser seguida para uma rede futura que permita atender aos requisitos das aplicações futuras em termos das qualidade de serviço (QoS - Quality of Service), mobilidade, segurança, experiência do usuário (QoE - Quality of Experience), entre outras características.

Alguns fatores podem ser enumerados para justificar mudanças nos padrões de comunicação atuais, em particular para a Arquitetura Internet, sendo que a possibilidade eminente de esgotamento das faixas de endereçamento do protocolo IP versão 4 (IPv4) e as limitações para o atendimento de aspectos da comunicação como endereçamento Multicast podem ser citados entre os principais catalizadores.

O presente capítulo aborda trabalhos relacionados a demandas das novas aplicações, a evolução das redes, a aspectos ligados ao endereçamento Multicast e a pesquisas experimentais em internet do futuro. 


\subsection{Aspectos de Endereçamento em Redes de Computadores}

Considerando que as comunicações ocorrem através de canais, então todos os participantes do canal devem ser endereçáveis para que não haja dúvida sobre sua origem (remetente) ou sobre seu(s) destino(s). Nesta seção é apresentada uma análise de trabalhos correlatos sobre os aspectos filosóficos, conceituais e arquiteturais de endereçamento em redes de computadores.

\subsubsection{Aspectos Conceituais e Filosóficos de Endereça- mento}

Comunicação (do Latim Communicare) tem como raiz a palavra "comum"e significa compartilhar. As entidades (seres) comunicantes têm em comum um canal de comunicação que pode ser físico, lógico ou virtual. Neste trabalho, o termo Primitiva significa o objeto das comunicações entre as entidades comunicantes.

Esta seção tem por objetivo apresentar as questões pertinentes ao endereçamento e, portanto, não se alonga em todos os aspectos da comunicação que podem ser encontrados em (SHANNON; WEAVER, 1949).

Entidades não se comunicam diretamente umas com as outras, mas através do canal que as enlaça, ie, quando duas ou mais entidades querem se comunicar, então elas escolhem um canal que será utilizado como o meio para troca de primitivas.

Por exemplo, no mundo humano, a comunicação entre duas ou mais pessoas se dá através de um canal físico com largura de banda de $20 \mathrm{kHz}$. Quando uma pessoa quer comunicar algo, ela faz uso (fala) do canal que se 
incumbe de levar o sinal sonoro até o(s) destino(s) (audição).

Desde os primórdios das redes, considerando que todas as entidades se tornaram passíveis de receber todas as primitivas enviadas através do canal compartilhado assim, foi introduzido um elemento que distinguisse uma entidade das demais - seu endereço. Endereçamento se tornou, portanto, um aspecto fundamental para as comunicações em ambientes de redes.

O termo LAN (Local Area Network) foi definido para estabelecer o âmbito de canais físicos (e respectivas tecnologias) e para limitar o espaço de endereçamento de entidades denominadas simplesmente de nós (nodes) da rede local. Ou seja, no âmbito das LANs, o endereçamento se dá no nível de máquinas (nós da rede).

A rigor, essa foi uma solução de compromisso levando-se em consideração: (i) as tecnologias de LANs disponíveis à época; e (ii) a arquitetura das máquinas. Nesse momento da tecnologia era fundamental que houvesse na placa de rede, um filtro que limitasse a quantidade de primitivas que chegassem ao barramento de sistema. Esse é o principal motivo pelo qual a subcamada MAC (Medium Access Control) reside na placa de rede conforme mostra a Figura 2.

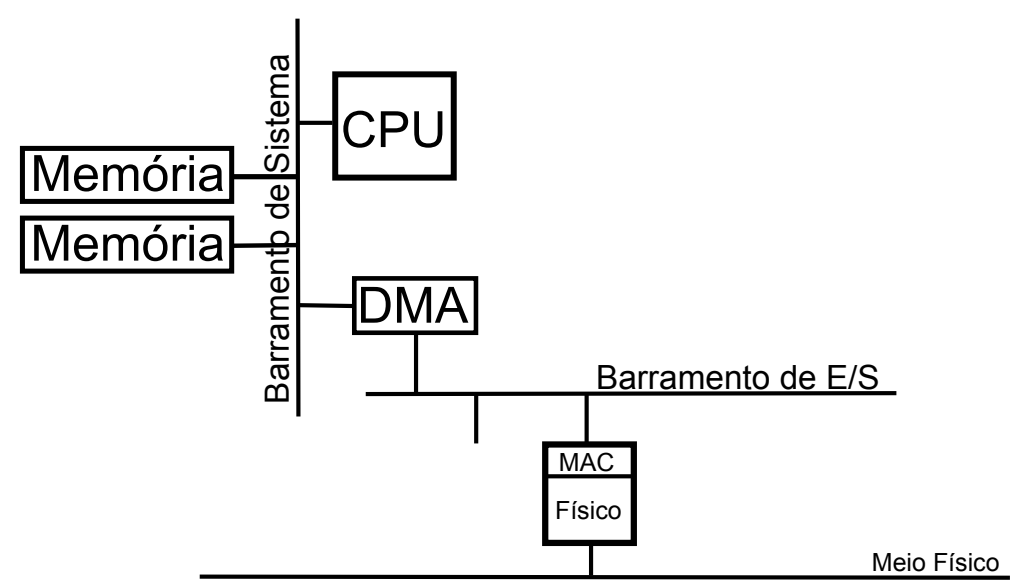

Figura 2: Arquitetura dos Primeiros Computadores. 
Observe-se na Figura 2 que se não houvesse um esquema de endereçamento na placa de rede, todos os sinais que transitassem pelo meio físico seriam passíveis de ocupar o barramento de E/S (inclusive as primitivas endereçadas a outras máquinas da rede) e atingir o barramento de sistema.

$\mathrm{Na}$ arquitetura Internet um nó da rede (host) é endereçado duas vezes, uma na camada de enlace (Endereço MAC) e outra na camada de rede (O endereço IP tem uma porção que endereça o host). Desse modo, um nó da rede deve manter sincronizados esses dois endereços, sob pena de não funcionar adequadamente. Note-se que ao se considerar as tecnologias atuais, os switches podem chavear um frame (primitiva) diretamente para a porta que liga o nó de destino e, portanto, o frame não é enviado a nós não endereçados. Desta forma um switch pode utilizar um endereço MAC, IP, ou outro critério (veja a Seção 2.2.3) para chavear os frame.

Considerando os requisitos de comunicação de um determinado conjunto de entidades (por exemplo, estações de trabalho em uma rede local de computadores), foram criados três tipos de endereçamentos: Unicast - que estabele uma relação biunívoca entre um endereço e uma entidade (Figura 3.a); Multicast - que estabelece uma relação unívoca entre um endereço e um subconjunto das entidades (Figura 3.b); e Broadcast - que estabelece uma relação unívoca entre um endereço e todas as entidades do conjunto (Figura 3.c).

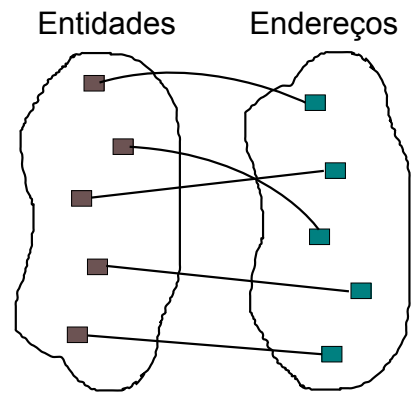

(a)

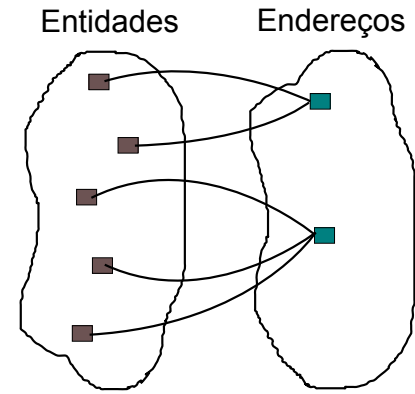

(b)

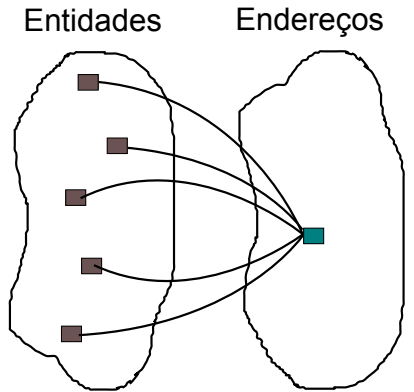

(c)

Figura 3: Endereçamentos: (a) Unicast (b) Multicast (c) Broadcast. 
Tomando-se por base as relações de conjuntos, é possível deduzir que endereços Unicast e Broadcast são casos particulares, sendo interessante observar que de fato Multicast é o tipo de endereçamento que é um super conjunto (ou generalização) dos tipos de endereçamentos (DAY, 2008). Por exemplo, observe-se o esquema de endereçamento MAC definido pelo comitê IEEE 802 para redes locais de computadores.

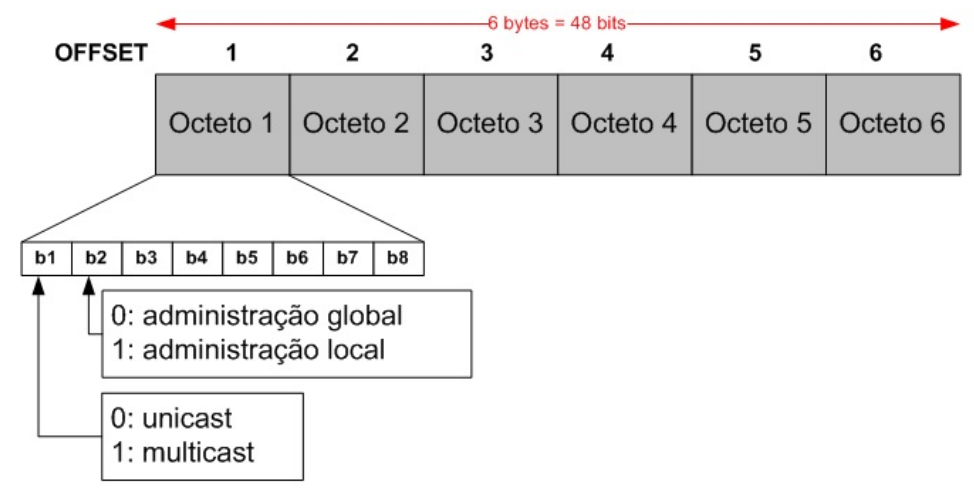

Figura 4: Endereçamento MAC IEEE 802.3.

Na Figura 4 é possível observar que o padrão de bits especificado pelo padrão IEEE 802.3 permite expressar todos os tipos de endereçamentos através de um endereço Multicast. O esquema de endereçamento IP reserva uma classe especialmente para endereços Multicast, sendo que abordagens similares podem ser feitas para os esquemas de endereçamento IP. Endereçamento na camada de Rede é abordado na seção 2.1.2.

Essa observação é fundamental para os objetivos deste trabalho, pois o esquema de endereçamento Multicast oferece as características necessárias para os requisitos de acessibilidade a conteúdos baseados em Video Conferência, Video sob Demanda, Televisão Digital, Dispositivos Móveis, Alive Streams, sem se esquecer das aplicações Web, entre outras. Endereçamento na camada de Aplicação será abordado na seção 2.1.3.

É interessante ressaltar que quando um usuário acessa a rede, ele o faz pensando em serviços, aplicações, conteúdos, entre outros, ou seja, na 
grande maioria das vezes não se faz acessos a rede pensando-se em máquinas, servidores, dispositivos, etc.

Contudo, os esquemas de endereçamentos, adotados para o momento da tecnologia que se inicou na década de 70 , são muito limitados para as necessidades atuais e futuras, pois, ao atrelar o endereçamento Multicast a partir da placa de rede, introduziu limitações para que um determinado nó (por exemplo uma estação de trabalho) hospede aplicações que pertençam a diferentes grupos (diferentes endereços Multicast). Oportunamente esse aspecto é discutido em maior profundidade.

\subsubsection{Endereçamento Multicast na Camada de Rede}

Do ponto de vista conceitual, o endereçamento na Camada de Rede deveria ser limitado a endereços de rede e, portanto, não deveria se preocupar com o endereçamento do nó (cite Modelo OSI - X.200). Contudo, por limitações técnicas, arquiteturas tais como X.25, BitNet e atualmente a arquitetura Internet projetaram endereços de rede que endereçam redes (Netld) e nós (Hostld).

Essa decisão de projeto atende à funcionalidade de mapeamento do endereço de Rede no endereço de Enlace (de facto endereço MAC), que é um requisito do Modelo de Referência OSI. Observe-se que essa decisão de projeto traz para a camada de rede parte das limitações apresentadas na seção 2.1.1. Para o escopo deste capítulo, essa análise é restrita ao esquema de endereçamento definido pelo Protocolo IP.

O Protocolo IP especifica três classes de endereçamento Unicast (Classes de endereços A, B e C) e uma classe de endereçamento Multicast (Classe D). Maiores detalhes sobre o esquema de endereçamento definido pelo Protocolo 
IP pode ser encontrado em (POSTEL, 1981b). O advento do CIDR (Classless Inter-Domain Routing), no início dos anos 90, permitiu que as classes de endereços Unicast fossem manipuladas mais facilmente, mas não atingiu a classe de endereços Multicast (GROUP; HINDEN, 1993b)(FULLER; LI, 2006).

Ao se assumir que um nó da rede pertencerá a um grupo Multicast, então serão necessários dois mapeamentos: a) um mapeamento entre os endereços Unicast MAC e IP; e b) um mapeamento entre os endereços Multicast MAC e IP.

Considerando que as placas de rede suportam apenas um endereço MAC de cada tipo (Unicast, Multicast e Broadcast), então isto limita a que um nó hospede aplicações que pertençam a grupos Multicast diferentes conforme discutido na seção 2.1.1.

No início dos anos 90, foi desenvolvido, por Van Jacobson, Steve Deering e Stephen Casner, um backbone experimental denominado de MBone (Multicast Backbone), para transmissão Multicast através da Internet (HANNA; PATEL; SHAH, 1999). O objetivo do MBone é minimizar a quantidade de dados requeridos para uma transmissão multiponto, por exemplo, de Audio/Video Conferência.

MBone se utiliza de mrouters que podem suportar Multicast IP e viabilizam o acesso a aplicações multimídia interativas via Internet. Todavia, como muitos roteadores da rede Internet desabilitam a transmissão de Multicast IP, devido à preocupação com o uso da largura de banda (Bandwidth) e tarifação, o MBone se limitou a conectar redes com capacidade de endereçamento Multicast usando a infraestrutura existente da Internet.

Outro fator limitante para o MBone é que a comercialização de roteadores com capacidade Multicast é limitada pelo fato de não haver um mecanismo 
eficiente de controle de acesso às árvores Multicast (Multicast trees), que incluem roteadores e os respectivos protocolos, e também porque os provedores de acesso à Internet têm dificuldade em controlar e tarifar este tipo de tráfego.

Apesar do Protocolo IP contar com uma classe para endereçamento Multicast, é notório o desuso dessa classe de endereços na camada de rede da Arquitetura Internet basicamente por três principais razões:

- limitação de um endereço por placa de rede - que proíbe a existência de duas ou mais aplicações pertencentes a mais de um grupo Multicast;

- dificuldade de alteração de endereço em regime permanente - a reconfiguração de grupos Multicast é restrita a intervalos de manutenção do equipamento;

- provedores de internet desabilitam o encaminhamento de tráfego Multicast - por motivos ligados a segurança, controle do uso da banda de comunicação, tarifação, etc.

Desse modo, a granularidade mínima de endereçamento oferecida pela Camada de Rede (nó de uma rede), independentemente do protocolo, não permite que se especifique o endereço de conteúdo, serviço ou aplicação. Este fato é determinante para que o mecanismo de endereçamento Multicast oferecido pelo Protocolo IP não seja praticamente utilizado.

Apesar dos motivos para introdução do IPv6, as razões listadas anteriormente se aplicam a essa versão do Protocolo IP no que tange a esses quesitos. De um modo geral, tráfego Multicast passou a ser tratado na Camada de Aplicação (ALM - Application Layer Multicast) (ver seção 2.1.3). 


\subsubsection{Endereçamento Multicast na Camada de Aplicação}

Claramente, em meios físicos inerentemente por difusão (Broadcast), é fácil se obter o endereçamento Multicast. Entretanto, Multicast se torna um problema complexo à medida que se distancia do meio físico. Em particular, quando a granularidade do endereçamento tende a seu mínimo, como é o caso de endereçamento de conteúdo, por exemplo, em geral calcula-se uma árvore de cobertura (spanning tree) mínima a partir do grafo da rede. O roteamento entre a origem e o(s) destino(s) é feito, na maioria dos casos, a partir da origem.

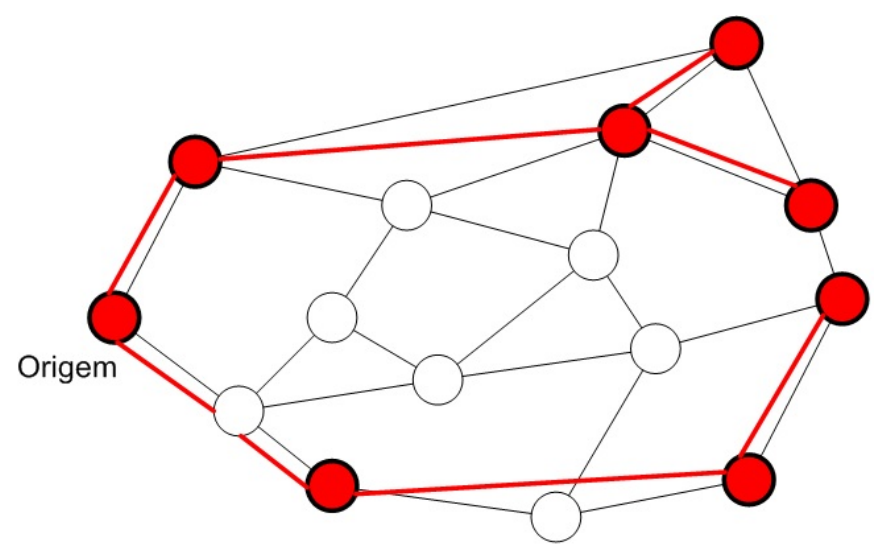

Figura 5: Membros do Grupo Multicast e Árvore de Cobertura com Raiz na Origem.

Uma primitiva enviada atravessa a árvore de cobertura, sendo replicada somente nos nós ramos (branching nodes) da árvore. Isso diminui consideravelmente o uso da banda de comunicação. Utilizando-se endereçamento Unicast, para se enviar uma determinada primitiva para $\mathrm{K}$ destinos, normalmente seriam requeridas $\mathrm{K}$ transmissões. Com endereçamento Multicast, $\mathrm{K}$ primitivas são entregues em $\mathrm{K}$ destinos, mas a rede como um todo não tem de ser sobrecarregada com $\mathrm{K}$ transmissões.

Algoritmos para árvore de cobertura distribuídos foram desenvolvidos há 
várias décadas, mas o aspecto de multipeer ${ }^{1}$ muda o problema. Considerando que a primitiva é reenviada a cada nó, diferentemente, um algoritmo distribuído deve rotear através da árvore em cada nó (ie, cada nó da árvore passa a ser um enviador) e deve ser encontrada uma árvore de cobertura mínima que seja ótima para um grupo de enviadores. Na camada de aplicação, essa capacidade recebe o nome de Application Layer Multicast (ALM).

Embora muitos encarem multicast como um outro modo de computação distribuída, em última análise, é um mecanismo para economizar o uso da banda de comunicação. É particularmente interessante para provedores de rede, pois Ihes permite 'desempenhar' mais tráfego (e geri-lo melhor) usando menos recursos. Termina aqui a simplicidade do problema de endereçamento Multicast na camada de aplicação.

Existe uma miríade de complicações, cujo desafio intelectual tem gerado extensa literatura sobre o assunto. Entretanto, multicast não encontrou muita adoção fora da comunidade de pesquisa (DAY, 2008). Como abordado em 2.1.2, a complexidade (e pouca flexibilidade) em configurar grupos Multicast, combinado com o aumento de velocidade das redes, fez com que a 'abordagem da força-bruta' (de enviar K cópias para K destinos) seja aceitável.

Como aplicações que utilizam Multicast continuam a se disseminar, por exemplo, em áreas como multimídia ou peer-to-peer, é de se esperar que, mesmo com os avanços em fibras ópticas, a necessidade de se utilizar a banda de comunicação com mais eficácia se imponha.

Muitas das pesquisas têm se concentrado em aplicações específicas e oferecido soluções para cada tipo de problema, com pouca ou nenhuma consideração sobre como essas soluções (particulares) funcionariam para outras

\footnotetext{
${ }^{1}$ Multipeer representa a forma mais geral de comunicação muitos-para-muitos que pode ser requerida para vários tipos de sistemas colaborativos(DAY, 2008)
} 
aplicações multicast com diferentes requisitos. Contudo, como multicast é um benefício antes de mais nada para os provedores de rede, é natural que eles (os provedores) queiram uma solução que atenda muitas aplicações multicast.

Busca-se o uso das experiências em redes tradicionais (tais como redes de dados) para trazê-las à arquitetura proposta neste trabalho com a finalidade de melhorar ou simplificar o problema de endereçamento das aplicações atuais e futuras.

O primeiro problema com Multicast (ignorado por muitos pesquisadores) (DAY, 2008) é que há muitas formas de multicast. É bastante fácil construir uma lista de características como as que seguem:

- Centralizado versus decentralizado (Multicast ou multipeer?);

- Entidades (população) estáticas versus dinâmicas (um membro pode mudar com o tempo?);

- Entidades conhecidas versus desconhecidas (membros podem ser desconhecidos com wireless?);

- Isotrópico versus anisotrópico (todos os membros se comportam do mesmo jeito?);

- Quorum (há um mínimo requerido de membros?);

- Confiável versus não confiável (serviços confirmados?).

Considerando essas características, há diversas políticas que poderiam ser usadas, fazendo com que a gama de potenciais protocolos seja suficientemente grande. Uma possibilidade é não esperar que uma única solução efetivamente acomode toda a gama. Contudo, criar soluções específicas para 
aplicações particulares pode levar à proliferação e complexidade na configuração da rede.

Tratar o endereçamento Multicast na camada de Aplicação (ALM) resolve diversas restrições introduzidas por esse tipo de endereçamento, originalmente nas camadas de Enlace e de Rede. Além disso, ALM atende ao requisito de diminuir o número de retransmissões de primitivas das aplicações e, por meio disso, otimizar o uso dos meios de transmissão (economia da banda de comunicação).

Contudo, um aspecto da manutenibilidade dificulta a adoção de ALM que é a dificuldade de reconfiguração da árvore de cobertura e a complexidade de mantê-la (senão intratável) para aplicações móveis.

\subsection{Ortogonalidade entre Planos de Controle e de Dados}

Em meados dos anos 80 , devido às potencialidades advindas da digitalização dos serviços de telefonia, a indústria de telecomunicações investiu pesadamente em um novo paradigma de serviços que se denominou Intelligent Network (IN) (AMBROSCH; MAHER; SASSCER, 1989).

Até esse momento, um nó da rede de telecomunicações (uma Central Telefônica, por exemplo) exercia dois papéis: (i) papel de controle da rede de telecomunicação - responsável por estabelecer/manter/encerrar conexões telefônicas; e, (ii) papel de comunicação - responsável por enviar/receber pacotes (primitivas) de voz ou dados.

Como esses papéis eram executados nos nós da rede, então cada fornecedor desenvolvia o seu sistema operacional proprietário e o embarcava em um equipamento cuja interface era proprietária (acesso ao terminal, lingua- 
gem, sistema operacional e arquitetura). O fato do papel de controle estar presente em todos os nós da rede dificultava imensamente a manutenção.

Mesmo se todos os equipamentos fossem de um único fabricante, ainda assim era necessário fazer uma atualização de configuração em todos os nós. A fim de não depender de um único fornecedor, as operadoras de telefonia contavam (e contam) com diversos equipamentos de diversos fornecedores, então o tempo de configuração ( Time to Market) e os custos (TCO - Total Cost of Ownership) eventualmente restringiam, sendo que muitas vezes tornavam proibitivo, o lançamento de um produto.

Por serem papéis ortogonais, IN (UNION, 1997) introduziu uma quebra de paradigma no qual esses papéis foram separados em dois planos, sendo os planos de Serviço (controle) e de Comunicação (dados). Essa separação somente foi possível com a padronização das interfaces dos planos e isto permitiu às operadoras se libertarem das particularidades dos fornecedores.

Do ponto de vista das redes de dados, no início dos anos 2000, percebeu-se que essas redes apresentavam características semelhantes àquelas de telecomunicações digitais dos anos 80 e que os equipamentos apresentavam as mesmas dificuldades de manutenção (ie, integração dos planos de controle e de dados em um único equipamento), momento em que a Intel publicou um trabalho que apresentava um novo paradigma para a separação dos elementos de controle e de envio de dados e que desde então denominou-se FORCES (Forwarding and Control Element Separation) (KHOSRAVl; ANDERSON, 2003)(DORIA et al., 2010)(HALPERN; SALIM, 2010).

FORCES define uma infra-estrutura arquitetural (e os respectivos protocolos) visando a padronização na troca de informações entre o Plano de Controle (Control Plane) e o Plano de Encaminhamento (Forwarding Plane) para os elementos de rede FORCES (FORCES NE). A comunicação entre os pla- 
nos da arquitetura FORCES foi padronizada através de um protocolo que permitiu ligar os elementos de rede a um controlador. Uma configuração feita no controlador era comunicada aos elementos de rede através do protocolo FORCES.

Subsequentemente, Ethane introduziu uma nova arquitetura de rede que permitia aos administradores definir uma política única, de granularidade fina, para toda a rede corporativa. Ethane conjuga switches Ethernet simples baseados em fluxos com um controlador centralizado, que gerencia a admissão e o chaveamento de fluxos. Apesar da nova abordagem, este projeto é compatível com as máquinas (hosts) e switches existentes (Backward Compatibility). Essa abordagem se popularizou através de SDN (Software Defined Network).

Esta seção reserva um espaço para aprofundamento na análise evolutiva da separação dos planos de controle e de dados que se iniciou nas Redes Inteligentes de Telecomunicações (seção 2.2.1), chegou às redes de dados através do FORCES (seção 2.2.2) e ganhou market place através das Redes Definidas por Software (seção 2.2.3).

\subsubsection{Redes Inteligentes em Telecomunicações}

Em meados dos anos 80, RBOCs (Reginal Bell Operating Companies) fizeram uma chamada para a caracterização de requisitos de uma nova rede de telecomunicações com os seguintes objetivos:

- disponibilização rápida de novos serviços na rede;

- independência de fornecedores e interfaces padronizadas; e,

- oportunidade para non-RBOCs oferecerem serviços para incrementar a utilização das redes. 
A Telcordia Technologies respondeu a essa chamada e desenvolveu o conceito denominado de Intelligent Network (IN) mostrado na Figura 6.

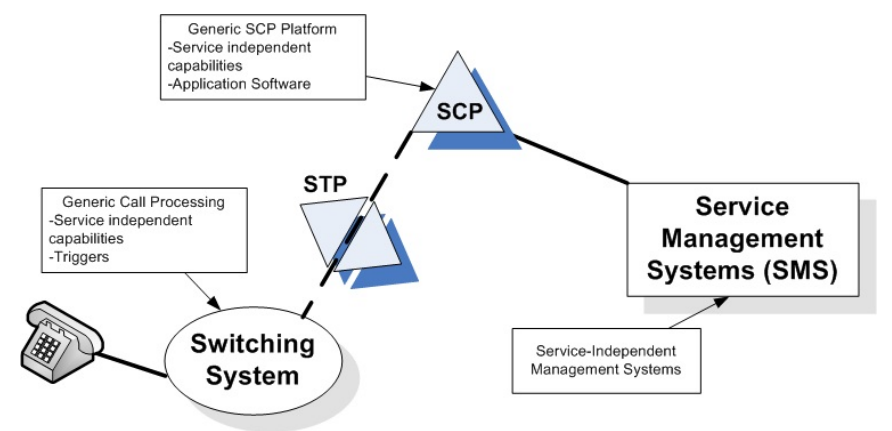

Figura 6: Visão Geral de Intelligent Network.

Até então, todas as funcionalidades de telefonia/telecomunicações residiam nos SSP (Service Switching Point) - na Figura 6 simplesmente denominado de Switching Systems - e nos STP (Signaling Tranfer Point). As regras para o encaminhamento de conexões (chamadas telefônicas, por exemplo) residiam em todos os nós da rede.

A introdução do conceito de IN marcou a primeira vez em que a lógica do serviço residia fora das centrais telefônicas (Switching Systems) e era armazenada (a lógica) em Entidades Físicas (PE - Physical Entity) denominadas de SCP (Service Control Point). Dois serviços evoluíram e fizeram uso da lógica de serviço IN - Serviço 0800 (FreePhone) e verificação de Cartões Telefônicos (Calling Card ou ABS - Alternate Billing Service).

Ressalte-se que até esse momento, as decisões para o estabelecimento de uma conexão telefônica (chaveamentos e encaminhamentos) eram tomadas em cada PE (nó da rede) e, a partir de IN, essas decisões passaram a ser tomadas no SCP e não mais nos SSP ou STP.

As regras de chaveamento/encaminhamento são mantidas pelo módulo de Gerenciamento de Serviços denominado de SMS (Service Management System). SMS tem uma visão da carga e da árvore de cobertura da rede de 
telecomunicações e, portanto, oferece ao SCP informações e regras a serem aplicadas nas conexões telefônicas. Além disso, SMS disponibiliza uma interface de operação que permite manutenção em regime permanente, em um único ponto, de toda a rede de telecomunicações sob sua égide.

A separação dos planos facilita enormemente a manutenção da rede e diminui drasticamente a complexidade dos nós da rede de telecomunicações. A simplificação, em função da retirada do Plano de Controle, e padronização (UNION, 1997) desses nós (SSP e STP) criou um segmento de mercado para hardware mais comuns e um espaço para que novas empresas ascedessem ao mercado, pois o hardware deixou de ser um produto específico de um fabricante e passou a ser uma commodity. A seguir são descritas as principais PEs de uma rede inteligente:

\section{a) SSP (Service Switching Point)}

Além de prover acesso a rede (uma central local) e desempenhar funcionalidades necessárias ao chaveamento (chamada), o SSP permite acesso a um conjunto de capacidades de IN. SSP é capaz de detectar requisições para serviços IN e contém capacidade de se comunicar com outras PEs, por exemplo um SCP (Service Control Point).

b) SCP (Service Control Point)

SCP contém programas com a lógica do serviço (SLP - Service Logic Program), que são usados para oferecer serviços IN, e podem, eventualmente, conter dados de clientes. Diversos SCPs podem hospedar os mesmos SLPs e dados para melhorar a confiabilidade dos serviços e, também, facilitam o balanceamento de carga (entre SCPs). Funcionalmente, um SCP contém uma função de controle do serviço (SCF Service Control Function) e pode, opcionalmente, conter uma função de 
serviço de dados (SDF - Service Data Function).

c) SDP (Service Data Point)

SDP contém dados usados pelos SLPs para oferecer serviços personalizados. Funcionalmente, um SDP contém uma função de dados de serviço (SDF - Service Data Function que pode ser acessada diretamente por um SCP ou um SMP, ou através da rede de sinalização. Pode, ainda, acessar outros SDPs na própria ou em outras redes.

d) SMP (Service Management Point)

SMP desempenha os controles para o gerenciamento, provisionamento e implantação dos serviços. Por exemplo, SMP pode administrar uma base de dados, fazer testes e supervisão da rede, gerenciar o tráfego da rede, e coletar dados da rede. Funcionalmente, SMP contém uma função de gerenciamento de serviço (SMF - Service Management Function) e pode acessar todos os outros tipos de PEs.

e) STP (Signaling Transfer Point)

STP oferece funcionalidades de roteamento, ie, ele roteia pacotes através da rede até determinado destino. Além de rotear, STPs coletam medidas da rede que podem ser utilizadas para monitorar e controlar o tráfego e uso da rede. STPs podem, ainda, fazer conversão de protocolos (desempenhar papel de gateway) como, por exemplo, para o protocolo de sinalização de VoIP (Voice over IP).

A Figura 7 apresenta a topologia e as relações dos principais elementos físicos (PE) de uma rede inteligente (IN) de telecomunicações.

O protocolo de comunicação entre o plano de controle e o plano de dados em uma Rede Inteligente de Telecomunicação é denominado INAP (Intelligent 


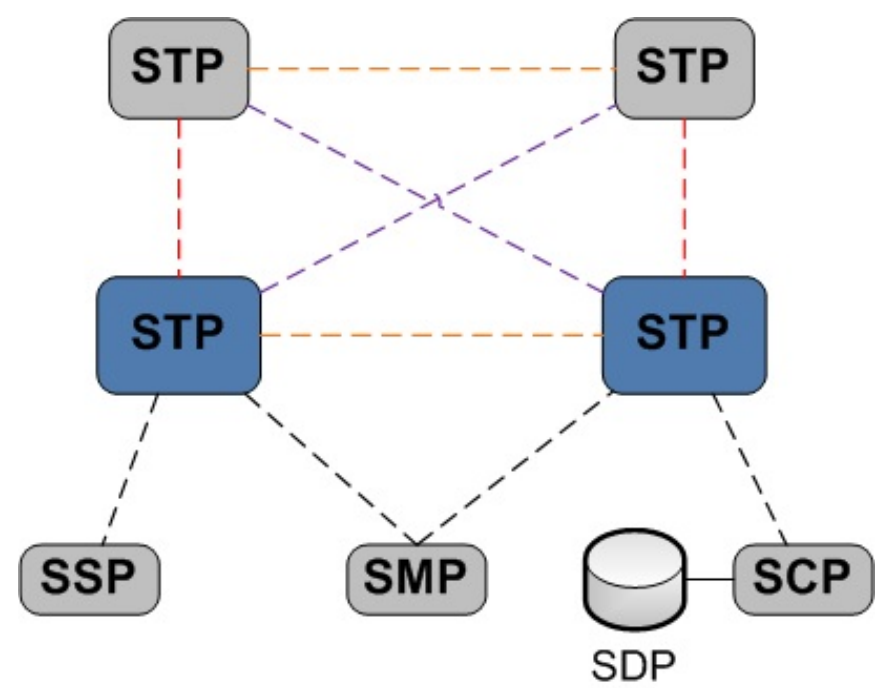

Figura 7: Elementos Físicos de Intelligent Network.

Network Application Protocol)(GURBANI; HAERENS; RASTOGI, 2005). Qualquer módulo que implemente os serviços INAP podem interagir com uma IN transparentemente.

\subsubsection{Visão Geral da Infraestrutura FORCES}

Esta seção objetiva introduzir os princípios da separação entre os elementos de controle (CE - Control Element) e os elementos de encaminhamento (FE - Forwarding Element), bem como oferecer uma visão resumida do protocolo FORCES (KHOSRAVI; ANDERSON, 2003)(DORIA et al., 2010)(HALPERN; SALIM, 2010) responsável pelas comunicações entre os elementos CEs e FEs.

Apesar de Redes Definidas por Software (SDN - Software Defined Network) ser o conceito do momento, FORCES pode ser considerada a precursora desse conceito. Em redes de dados, é a primeira vez que se menciona a separação entre os planos de dados e de controle.

FORCES define uma infraestrutura arquitetural e os respectivos protocolos com a finalidade de padronizar a troca de informações entre o plano de con- 
trole (composto de CEs) e o plano de encaminhamento (composto de FEs) em uma rede de elementos FORCES (FORCES NE). A infraestrutura FORCES é definida por (YANG et al., 2004), enquanto os requisitos são definidos por (KHOSRAVI; ANDERSON, 2003).

A especificação de FORCES FE (HALPERN; SALIM, 2010) apresenta os Blocos de Funções Lógicas (LFB - Logical Function Blocks) de um elemento FE utilizando XML. Os componentes de configuração, as capacidades e os eventos relacionados são definidos no momento em que um LFB é formalmente criado. LFBs existentes em um FE são controlados de forma padronizada através do protocolo FORCES.

A Figura 8 é reproduzida a partir do documento (YANG et al., 2004) para maior clareza. Ela mostra os elementos de rede FORCES com dois CEs e dois FEs, bem como as respectivas interfaces entre estes elementos.

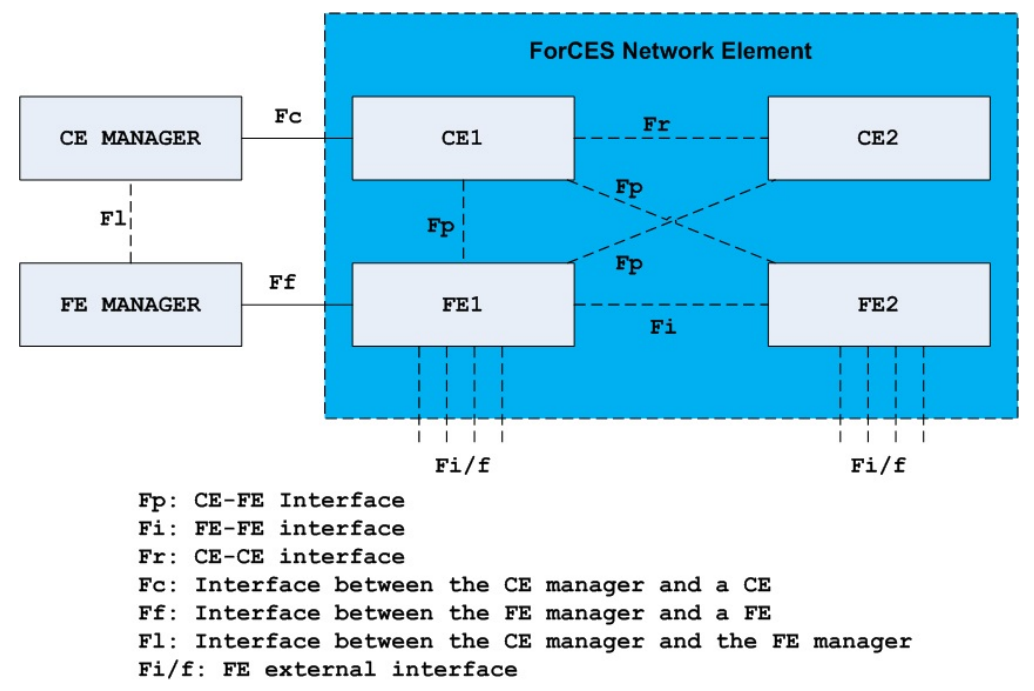

Figura 8: Diagrama da Infraestrutura FORCES (YANG et al., 2004).

É interessante observar que as interfaces previstas na Figura 8 são genéricas e permitem que quaisquer tecnologias e equipamentos de quaisquer fabricantes possam interagir através do protocolo FORCES.

O elemento de rede FORCES FE especifica a arquitetura de um disposi- 
tivo mínimo, genérico, que apenas executa as funções de encaminhamento, e cuja definição do comportamento de encaminhamento (rota ou caminho) é especificado pelos FORCES CE. FORCES CE é então o controlador do comportamento da rede e pode ser construído em diversas tecnologias de hardware, sistemas operacionais, liguagens, etc, sendo que o único requisito é que implemente a sua interface em acordo com o protocolo FORCES.

Por exemplo, a Figura 9 mostra uma interface do protocolo FORCES através de um elemento de rede que habilite QoS em MPLS (Multi Protocol Label Switching).

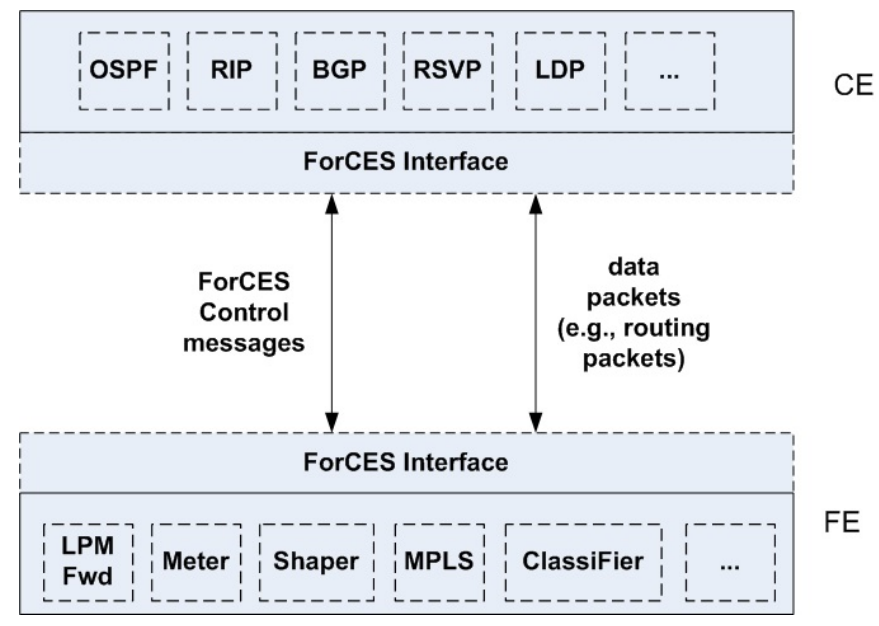

Figura 9: Interface Forces: funções CE e FE.

FORCES não se preocupou com aspectos de endereçamento e, portanto, quaisquer necessidades de endereços Unicast, Multicast ou Broadcast deveriam ser definidos por arquitetos e projetistas de uma solução baseada em FORCES. Outro aspecto que ficou fora do escopo do FORCES foi a comunicação sem fio.

\subsubsection{Redes Definidas por Software}

Software Define Networking (SDN) (GOTH, 2011) (GREENE, 2009) representa uma oportunidade extraordinária para repensar as redes de computado- 
res. Assim como IN e FORCES, SDN consiste de uma abstração que separa o software que controla os elementos da rede do hardware, oferecendo uma interface aberta e bem definida para controlar e modificar o comportamento desses elementos de rede em tempo de execução.

As bases conceituais de SDN foram apresentadas pela arquitetura 4D (GREENBERG et al., 2005) e refinadas pela arquitetura Ethane (CASADO et al., 2007). O conceito de SDN permite que novos protocolos sejam especificados e experimentados em condições reais, mesmo em regime permanente, ie, em redes de produção.

Atualmente o conceito de SDN é materializado através do OpenFlow (MCKEOWN et al., 2008). Essencialmente, o OpenFlow separa o plano de dados do plano de controle, definindo um OpenFlow Switch com uma arquitetura mínima, genérica, que apenas executa as funções de encaminhamento de dados. O OpenFlow está preparado para interagir com as redes atuais, porém ele pode ser utilizado para moldar e também implantar a arquitetura das redes do futuro. Neste contexto o SDN e o OpenFlow estão sendo utilizados por este trabalho, viabilizando a sua implantação e experimentação.

A implantação e a experimentação de novas arquiteturas de rede é algo de difícil execução, mesmo em um laboratório dentro de um campus. A experimentação de uma nova forma de endereçamento que não é baseada no protocolo IP, por exemplo, é uma tarefa complexa.

Outro aspecto importante em relação à área dessa pesquisa é que qualquer proposição, para que tenha aceitação na Internet, deve considerar as escalas apropriadas durante a experimentação, o que consiste em uma complexidade adicional para a implantação em um laboratório.

Neste cenário, no qual os pesquisadores estão presos em suas próprias 
redes, SDN fornece algumas abstrações que permitem separar o software, que controla os elementos de rede, do hardware que executa as funções de comunicação propriamente ditas, através de interface aberta e bem definida para controlar e modificar o comportamento do hardware, permitindo assim a implantação e experimentação de novas arquiteturas de rede.

Um switch OpenFlow, de acordo com o especificado a partir da versão 1.1 e conforme mostrado na Figura 10, consiste de: uma ou mais flow tables; uma group table; e um canal seguro para um controlador (Controller) externo. As tabelas são utilizadas na definição de qual ação um dado frame sofrerá. O controlador gerencia o switch utilizando o protocolo OpenFlow e controla seu comportamento modificando o conteúdo das flow tables bem como as entradas na group table.

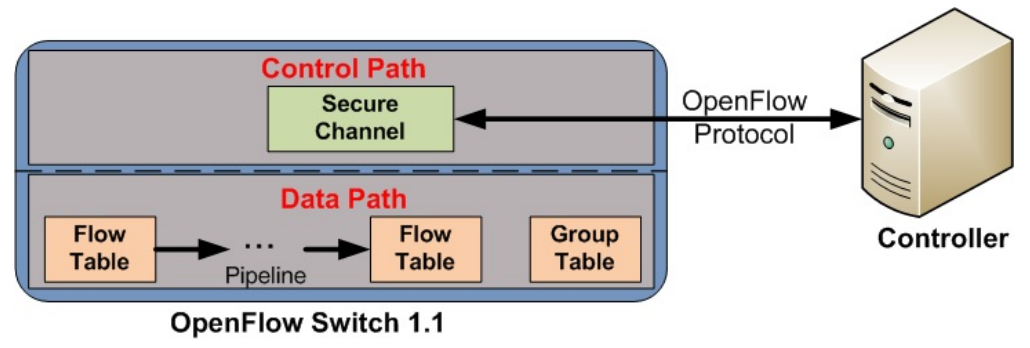

Figura 10: Componentes de um OpenFlow Switch (PFAFF et al., 2011).

A especificação 1.0 do OpenFlow considera uma única flow table, enquanto as versões $1.1,1.2$ e 1.3 consideram a existência de uma série de tabelas que são avaliadas em sequência. Cada entrada na flow table possui uma chave e um conjunto de ações associadas. A chave é composta de um conjunto de campos que basicamente correspondem aos cabeçalhos dos protocolos das camadas dois, três e quatro da arquitetura TCP/IP.

Ao receber um frame, os campos de seus cabeçalhos são extraídos e comparados com a chave e, caso combinem, a ação associada àquela chave será aplicada ao mesmo. 
Cada versão do OpenFlow possui uma lista de ações possíveis que podem ser aplicadas ao frame. Todas as versões suportam o seu descarte ou o seu encaminhamento para uma ou mais portas do switch. Quando não é encontrada uma correspondência entre um frame e uma entrada em alguma das flow tables, uma ação possível é encaminhá-lo para o Controller.

O comportamento do switch é governado pelo estado das flow tables e o Controller é o responsável pela manutenção desse estado em um ou mais switches.

\subsubsection{Especificações do OpenFlow}

A tabela 1 a seguir apresenta uma comparação entre as especificações do OpenFlow disponíveis até o momento. A versão 1.0 é amplamente suportada e vários switches baseados tanto em software quanto em hardware estão disponíveis. Para as versões 1.1 e 1.2 somente switches baseados em software estão disponíveis.

Os número de protocolos suportados em cada versão está diretamente ligado ao número de campos existentes na chave utilizada para identificar uma entrada na flow table.

A especificação 1.1 introduziu a noção do uso de novas estruturas, chamadas Flow Match Structures (conforme descrito na seção A.2.3 (PFAFF et al., 2011)), que permitem definir o número e o tamanho em bits de cada campo existente na chave da flow table. Esta noção, por sua vez, foi ampliada na especificação 1.2 através da chamada OpenFlow Extensible Match (OXM), conforme descrito na seção A.2.3 (FOUNDATION, 2011).

Através da OXM é possível definir novas classes para a chave utilizada na flow table. Em cada classe, utilizando um formato baseado em Type-Length- 
Tabela 1: Comparação entre as Versões do OpenFlow.

\begin{tabular}{|c|c|c|c|c|c|c|c|c|}
\hline Versão & $\begin{array}{c}\text { Data de } \\
\text { Lançamento }\end{array}$ & $\begin{array}{l}\text { Flow } \\
\text { Tables }\end{array}$ & $\begin{array}{l}\text { Número } \\
\text { de } \\
\text { Campos } \\
\text { na Chave }\end{array}$ & $\begin{array}{l}\text { Extensible } \\
\text { Match } \\
\text { Support }\end{array}$ & Disponibilidade & $\begin{array}{c}\text { Ações } \\
\text { Obrigatórias }\end{array}$ & Ações Opcionais & $\begin{array}{l}\text { Protocolos } \\
\text { Suportados }\end{array}$ \\
\hline 1.0 & $12 / 31 / 2009$ & 1 & 12 & No & $\begin{array}{c}\text { Software e } \\
\text { Hardware }\end{array}$ & $\begin{array}{c}\text { Forward, } \\
\text { Drop }\end{array}$ & $\begin{array}{c}\text { Forward }^{1}, \\
\text { Enqueue, } \\
\text { Modify-Field }\end{array}$ & Ethernet, IP, TCP \\
\hline 1.1 & $02 / 28 / 2011$ & Pipeline & 15 & No & Software & $\begin{array}{c}\text { Output, Drop, } \\
\text { Group }\end{array}$ & $\begin{array}{c}\text { Output }^{1} \text {, Set- } \\
\text { Queue, } \\
\text { Push-Tag/Pop-Tag, } \\
\text { Set-Field }\end{array}$ & $\begin{array}{c}\text { Protocolos da } \\
\text { Versão } 1.0+ \\
\text { MPLS }\end{array}$ \\
\hline 1.2 & $12 / 05 / 2011$ & Pipeline & 36 & Yes & Software ${ }^{2}$ & $\begin{array}{c}\text { Output, Drop, } \\
\text { Group }\end{array}$ & $\begin{array}{c}\text { Set-Queue, } \\
\text { Push-Tag/Pop-Tag, } \\
\text { Set-Field, } \\
\text { Change-TTL }\end{array}$ & $\begin{array}{l}\text { Protocolos da } \\
\text { Versão } 1.1+\text { IPv6 }\end{array}$ \\
\hline 1.3 & $04 / 16 / 2012$ & Pipeline & 40 & Yes & Não Disponível & $\begin{array}{c}\text { Output, Drop, } \\
\text { Group }\end{array}$ & $\begin{array}{c}\text { Set-Queue, } \\
\text { Push-Tag/Pop-Tag, } \\
\text { Set-Field, } \\
\text { Change-TTL }\end{array}$ & $\begin{array}{c}\text { Protocolos da } \\
\text { Versão } 1.2+ \\
\text { Extensões de } \\
\text { Cabeçalho IPv6 }\end{array}$ \\
\hline
\end{tabular}

-Value (TLV), é possível descrever campos da chave, dessa forma é possível tratar tanto os protocolos atuais como futuros o que interessa diretamente para a comunidade de pesquisa que atua com novas arquiteturas para Internet normalmente baseadas em pilhas de protocolos clean slate.

Um switch baseado em software de acordo com a especificação 1.2 foi disponibilizado recentemente (TELECOMUNICAçõES, 2012) e, além disso, um outro ainda em versão alfa (INFOBLOX, 2012) também foi disponibilizado.

A última especificação aprovada, a versão 1.3 (FOUNDATION, 2012), possui suporte completo ao protocolo IPv6 e está prevista para ser o foco da indústria para o próximos switches baseados em hardware (KERNER, 2012).

A seguir são apresentadas algumas primitivas definidas pelo protocolo OpenFlow. Pode-se afirmar que essas primitivas são as mais relevantes no modelo de comunicação entre o switch e o controller.

a) OFPT_PACKET_IN: Essa primitiva é utilizada pelo switch para enviar ao controller um pacote recebido. O envio do pacote ocorre quando não é possível encontrar na flow table uma entrada corresponde a ele. Esta 
mensagem é assíncrona e pode, ou não, estar habilitada no switch.

b) OFPT_PACKET_OUT: De maneira complementar, essa primitiva é utilizada para enviar um pacote do controller para o switch.

c) OFPT_FLOW_MOD: Utilizada pelo controller para modificar a flow table de um switch. Essa primitiva permite incluir, modificar e excluir uma entrada na tabela.

\subsubsection{Media Independent Handover Services}

O principal objetivo do padrão IEEE 802.21 (IEEE, 2009) é auxiliar no processo de handover entre diferentes tecnologias de acesso, permitindo assim que uma entidade conectada a uma rede de acesso, por exemplo, baseada na especificação IEEE 802.11, possa de maneira transparente conectar-se a outra rede, baseada por exemplo na tecnologia IEEE 802.16. Para isto o padrão IEEE 802.21 realiza uma abstração do controle dos enlaces de acesso, oferecendo às camadas superiores um controle agnóstico em relação à tecnologia. Essa abstração é realizada pela função chamada Multimedia Independent Handover Function (MIHF) que fornece os serviços definidos pelo protocolo (CORUJO et al., 2011).

A Figura 11 a seguir apresenta o modelo utilizado pelo IEEE 802.21. O LINK_SAP abstrai o controle do enlace de uma determinada tecnologia. A função MIHF recebe eventos específicos de um enlace (LINK Events) e atua sobre esse enlace utilizando a seu conjunto próprio de comandos (LINK Commands). Um usuário do modelo previsto na especificação IEEE 802.21 (MIH USER) recebe eventos genéricos (MIH Events) e atua sobre os links utilizando comandos independentes da tecnologia do link de acesso (MIH Commands).

A comunicação entre entidades MIH ocorre através do protocolo MIH uti- 


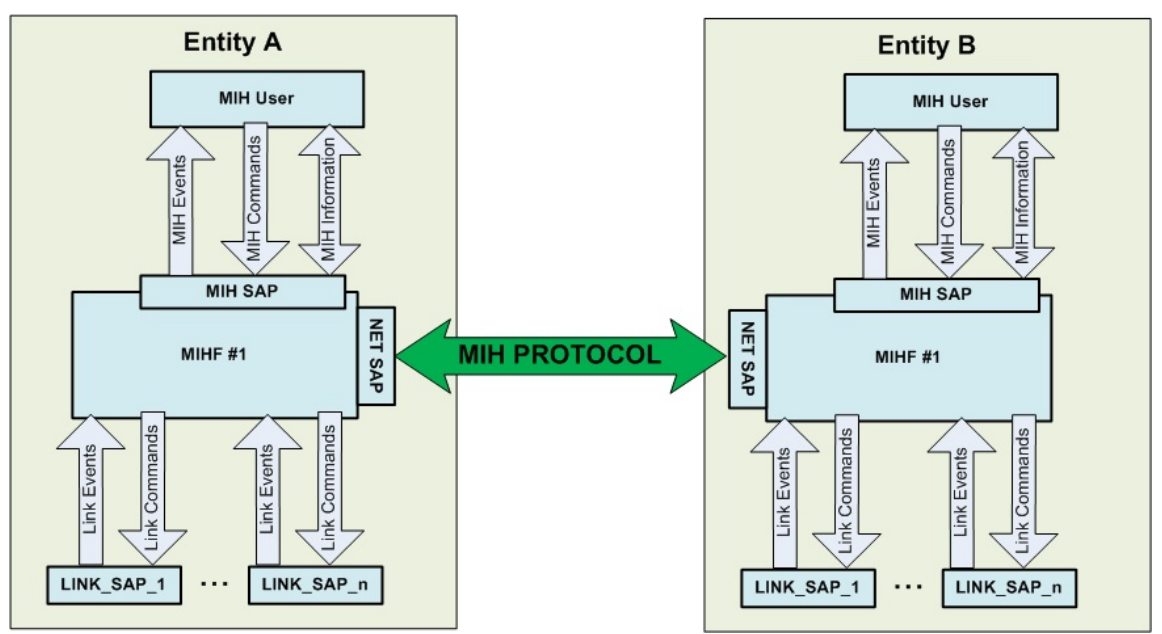

Figura 11: Componentes do Modelo MIH (IEEE, 2009).

lizando para isso um ponto de acesso ao serviço da rede (NET SAP). O protocolo MIH possui serviços para diferentes tipos de handover sendo que este processo pode ser iniciado por um nó móvel (Mobilie Node - MN) ou então pela própria rede. Por exemplo, um handover iniciado pelo MN utiliza as seguintes primitivas de serviço:

a) MIH_MN_HO_Candidate_Query: utilizado pelo MN para submeter à rede uma consulta por possíveis candidatos a fim de iniciar um handover. O MN pode sugerir à rede uma lista de possíveis candidatos para a rede. A resposta indica a lista de redes de acesso, em ordem de preferência, para onde o início do processo handover pode ser considerado pelo $\mathrm{MN}$.

b) MIH_MN_HO_Commit: Utilizado pelo $\mathrm{MN}$ a fim de notificar a rede de acesso de destino para onde efetivamente iniciará um processo de handover.

c) MIH_MN_HO_Complete: Utilizado pelo $\mathrm{MN}$ a fim de notificar à rede que concluiu o processo de handover. 


\subsubsection{Comparação entre Tecnologias de Enlaces}

A presente seção tem o objetivo de comparar as arquiteturas e conceitos de enlaces apresentadas nas seções anteriores considerando os seguintes critérios:

a) Plano de Controle

Apesar de todas as arquiteturas e conceitos mencionados nesta seção terem como motivação a separação entre os planos de controle e de dados, este critério é citado para fixação do conceito.

b) Enlace

Este critério aparece para explicitar que outras tecnologias de Enlace são passíveis de separação dos planos de controle e de dados, e não apenas a tecnologia Ethernet.

c) Cabeçalho (Header) Considerando o formato tradicional das primitivas, principalmente as de enlace, que contam com Header, Body (ou Payload) e Trailer (opcional), o cabeçalho conta com campos rigidamente definidos e, portanto, não passíveis de alteração. Contudo, OpenFlow 1.3 permite que os campos do cabeçalho sejam definidos e, então, novos protocolos de enlace poderão ser experimentados (ou criados).

d) Fluxos Switches recebem este nome principalmente por atuarem como uma chave ao analisarem os frames de suas filas de entrada. Contudo, diversas tecnologias tratam os frames independentemente uns dos outros - sem memória (Stateless), enquanto outras permitem uma configuração de sessão (Statefull). O termo Fluxo tem a semântica de tecnologias que tratam os frames de acordo com uma sessão que pode ser um circuito lógico (Logial Circuit) ou um fluxo (Flow) no conceito OpenFlow. 
e) QoS (Quality of Service)

Este critério é importante nas redes atuais, pois quesitos tais como vazão (troughput), largura de banda (bandwidth), tempo real (realtime), regularidade no atraso (release jitter), entre outros, são fundamentais nas aplicações multimídia. Nesta seção está-se interessado se a tecnologia suporta algum requisito de QoS ou simplesmente não suporta QoS.

f) QoE (Quality of Experience)

QoS é característica cada vez mais frequente nas aplicações, entretanto, a experiência do usuário (que pode incluir o tipo de dispositivo que o usuário manuseia no momento do acesso) também é um critério interessante pois pode ter como consequencia um melhor uso da infra-estrutura e pode até significar redução no consumo de energia. Assim como no caso de QoS, nesta seção está-se interessado se a tecnologia suporta ou não algum quesito de QoE.

Tabela 2: Quadro Comparativo Entre as Tecnologias de Enlaces

\begin{tabular}{|c|c|c|c|c|c|c|}
\hline Tecnologia & \multirow{2}{*}{ IN } & \multirow{2}{*}{ FORCES } & \multirow{2}{*}{ 4D } & \multirow{2}{*}{ SDN } & \multicolumn{2}{|c|}{ OpenFlow (OF) } \\
\hline Critério & & & & & OF 1.0 & OF 1.3 \\
\hline Plano Controle & $\operatorname{sim}$ & $\operatorname{sim}$ & $\operatorname{sim}$ & $\operatorname{sim}$ & $\operatorname{sim}$ & $\operatorname{sim}$ \\
\hline Enlace & ISUP & Genérico & Genérico & Genérico & Ethernet & Ethernet \\
\hline Cabeçalho & Fixo & Fixo & Fixo & Fixo & \begin{tabular}{|l|} 
Fixo \\
\end{tabular} & Configuráve \\
\hline Fluxos & Sim & Não & Não & Sim & Sim & Sim \\
\hline Qos & Sim & Não & Não & Não & Não & Não \\
\hline QoE & Não & Não & Não & Não & Não & Não \\
\hline
\end{tabular}

A Tabela 2 apresenta um quadro comparativo que permite visualizar o suporte que as tecnologias oferecem aos critérios expostos nesta seção.

É interessante observar que embora OpenFlow 1.3 se baseie na LAN Ethernet, o fato de permitir que se defina o formato do cabeçalho para fins de chaveamento do frame, então esta versão se veste de uma enorme promessa para a Internet do Futuro, restando abertos os aspectos de QoS e QoE. 


\subsection{Pesquisa Experimental em Arquiteturas para a Internet do Futuro}

A capacidade de alterar o comportamento do switch em tempo de execução, através da modificação do estado da flow table, fornece ao operador da rede uma gama de novos controles permitindo o surgimento de novos serviços sobre as redes atuais, especialmente aquelas localizadas em campi (NAOUS et al., 2008), empresas (KOPONEN et al., 2010) e data centers (Al-Fares et al., 2010).

Atualmente, na Internet, requisitos de comunicação como multihoming, segurança, mobilidade e multicast são questões abertas e vários grupos de pesquisa estão envolvidos no projeto da próxima geração da Internet que visa satisfazer esses requisitos das aplicações. Neste contexto, o OpenFlow é uma tecnologia que permite apoiar a pesquisa experimental com foco na Internet do futuro.

\subsubsection{Instalações Experimentais}

O Openflow está viabilizando a pesquisa experimental apoiada em grandes testbeds que propiciam aos pesquisadores a oportunidade de utilizar as escalas apropriadas em seus experimentos, conforme necessário para uma arquitetura que visa a Internet do futuro. Este processo é semelhante ao que ocorreu no surgimento da Internet atual em que o seu desenvolvimento foi baseado na utilização da ARPANET (CATLETT; TOOLE, 1998).

Patrocinada pela NSF, a GENI (ELLIOTT, 2010) é uma instalação experimental que conecta campi distribuídos pelos Estados Unidos permitindo que pesquisadores, tanto da academia quanto da indústria, possam executar em grande escala, experimentos cujo foco seja a inovação nas redes de compu- 
tadores. Atualmente em sua espiral cinco, o projeto GENI em conjunto com a Internet2 e o National Lambda Rail (NLR) estão implantando o OpenFlow em todos os seus backbones, disponibilizando uma rede onde o uso de SDN é nativo e, desta forma, está preparada para a implantação e experimentação de novos serviços, oferecendo aos pesquisadores recursos que permitem explorar novas arquiteturas para a Internet do Futuro.

Na Europa, o projeto OFELIA (OpenFlow in Europe Linking Infrastructure and Applications) (OFELIA, 2011), financiado pelo FP7 (Seventh Framework Programme), está construindo uma instalação experimental baseada em OpenFlow que interconecta oito ilhas em diferentes países. Essa instalação, que possui um substrato baseado em SDN, está disponível ao público. Com uma estratégia baseada em Open Calls, o projeto oferece recursos para que novos parceiros possam se juntar ao OFELIA trazendo novas ideias que visem a melhoria da infraestrutura bem como realizar novos tipos de experimentos.

No Brasil, o projeto FIBRE (Future Internet Experimentation Between Brazil and Europe) (ABELEM et al., 2011) tem por objetivo criar e implantar uma testbed baseada em OpenFlow que interconectará diferentes instituições de ensino e que será federada com o projeto OFELIA na Europa. Além disso, um dos objetivos da proposta EDOBRA (Extending and Deploying OFELIA in BRAzil) (ATNOG, 2012) é implantar no Brasil uma ilha do projeto OFELIA. Essas duas iniciativas criarão uma infraestrutura intercontinental baseada em OpenFlow que auxiliará a pesquisa experimental em novas arquiteturas de rede. 


\subsubsection{Novas Arquiteturas para a Internet do Futuro}

Diversos grupos de pesquisa estão envolvidos na pesquisa cujo foco são novas arquiteturas de rede que atendem aos requisitos da Internet do Futuro. Os esforços descritos neste trabalho possuem em comum a visão de uso de uma nova pilha de protocolos, bem como novas abordagens para identificação e endereçamento.

Nos Estados Unidos o projeto NSF FIA (Future Internet Architecture) (FOUNDATION, 2010) financia cinco diferentes iniciativas que visam projetar e avaliar novas arquiteturas para a Internet. Dessas, duas possuem visões que são correlatas com este trabalho de pesquisa: os projetos MobilityFirst (ver Subseção 2.3.2.1) e eXpressive Internet Architecture (ver Subseção 2.3.2.2)

$\mathrm{Na}$ Europa, quase uma centena de projetos, financiados pelo programa FP7, dentro de seu objetivo 1.1 (A Rede do Futuro) estão diretamente relacionados com a Internet do Futuro como por exemplo: 4WARD, CHANGE, MEDIEVAL, PURSUIT, SAIL, SENSEI, TRILOGY and UNIVERSELF (COMMISSION, 2012c). No geral esses projetos baseiam-se em uma abordagem clean slate e endereçam diferentes requisitos da Internet do Futuro.

No Brasil o grupo de pesquisa que suporta este trabalho está projetando uma nova arquitetura de rede com foco na Internet do futuro onde um controlador centralizado lida com aspectos da rede como identificação, endereçamento, mobilidade, controle de tráfego e de sessão. Como essa visão encaixa-se perfeitamente no conceito de SDN, o seu uso foi adotado e aspectos dessa arquitetura estão sendo experimentados com o OpenFlow. A pesquisa, descrita neste documento, contudo, possui como foco um protocolo que suporte um novo modo de encaminhamento de pacotes.

A seguir é apresentada uma descrição de dois desses projetos ressaltando 
alguns aspectos centrais das arquiteturas de rede propostas.

\subsubsection{MobilityFirst}

O projeto MobilityFirst (SESKAR et al., 2011) (NELSON; BHANAGE; RAYCHAUDHURI, 2011) argumenta que a mobilidade é o objetivo fundamental quando se trata da Internet do futuro. Para atingir este objetivo uma nova arquitetura de protocolos está sendo proposta e alguns de seus aspectos chave são: uma nova abordagem para identificação baseada em um GUID (Globally Unique Identifier) que é mapeado em um endereço de rede plano (NA); e, um roteamento com capacidade de armazenamento que pode suportar uma visão de Internet centrada em conteúdo (Content-Centric) e que auxilia durante os processos de mobilidade através do armazenamento dos dados pelo tempo necessário, para posterior entrega ao usuário.

Um componente da arquitetura, chamado de GSTAR (Generalized Storage-Aware Routing) consiste de um mecanismo que suporta a variação da qualidade da rede de acesso, bem como a sua interrupção garantindo a entrega dos dados nas mais variadas situações.

Por exemplo, caso em um dado momento, um usuário esteja utilizando um acesso de boa qualidade e possa ser encontrado na rede, os dados são entregues em tempo de switching através do GSTAR. Na presença de uma rede de acesso de baixa qualidade ou mesmo durante uma desconexão, em um processo de troca entre redes de acesso, por exemplo, o mecanismo do GSTAR poderia adaptar-se a essa situação e passar a atuar em um modo store-and-forward. Nesse caso os dados seriam armazenados para entrega em um momento posterior quando o usuário, ainda que esteja utilizando um novo endereço de rede (NA), é identificado pelo mesmo GUID. Finalmente o mecanismo do GSTAR poderia suportar um período mais longo de desconexão, 
armazenando os dados e entregando-os apenas em um momento posterior, neste caso a rede atuaria como uma DTN (Delay Tolerant Network).

A arquitetura também considera um novo serviço, chamado GRNS (Global Name Resolution Service), que é distribuído ao longo dos roteadores e cuja responsabilidade é mapear o GUID em um endereço de rede (NA). No caso de uma troca de rede, o NA é alterado, porém o GUID permanece o mesmo, permitindo que a nova rota seja calculada. As primitivas utilizadas na arquitetura contém em seu cabeçalho tanto do GUID, como o NA, que é utilizado em um primeiro momento para rotear os pacotes. Quando não é mais possível o roteamento pelo NA, o GNRS executa uma resolução a fim de calcular o novo NA associado ao mesmo GUID, possibilitando a entrega a um usuário móvel e resolvendo somente quando necessário. Um GUID pode ser associado a um usuário, dispositivo, conteúdo ou também a um contexto. Em oposição à visão da rede centrada em conteúdo, na arquitetura MobilityFirst, atributos do conteúdo não são transmitidos nos cabeçalhos dos pacotes.

O projeto possui três fases que são baseadas na prototipação. Na primeira fase o protótipo foi construído utilizando o Click (KOHLER et al., 2000), um roteador baseado em software. A segunda fase considera experimentos em pequenas escalas com a implantação de uma pilha que suporte os protocolos do MobilityFirst e os serviços associados. Na terceira fase, planejada para 2013, a implantação será realizada em larga escala utilizando a GENI.

Apesar do Click ter sido utilizado para o primeiro protótipo, a equipe do projeto está construindo outro baseado em NetFPGA (NAOUS et al., 2008) e dessa forma habilitado para uso com OpenFlow. Nesse novo protótipo, os componentes responsáveis pelo roteamento e gerenciamento da rede serão implantandos no ambito do controlador do OpenFlow. 


\subsubsection{2 eXpressive Internet Architecture}

A eXpressive Internet Architecture (XIA) (HAN et al., 2012) é uma arquitetura baseada na abordagem clean slate, em que a segurança e a capacidade de evolução são seus princípios básicos. De acordo com a visão dessa arquitetura, sua entidade é chamada Principal, que pode ser um conteúdo, um serviço, um host ou um usuário. Esta visão representa uma abordagem *-centric da rede. A arquitetura utiliza um novo esquema de identificação baseado um hashing de uma chave pública associada ao Principal, o que na visão do seus proponentes, agrega à rede capacidades intrínsecas relacionadas com integridade e não-repúdio.

A arquitetura define um novo esquema de endereçamento baseado em um DAG (Directed Acyclic Graph) que contém a identificação de um grupo de Principals em cada hop, por exemplo, o endereço de um conteúdo poderia ser expresso como um DAG que contém os Principals do domínio (rede de destino), do host, do serviço e finalmente do conteúdo. Os arcos desse grafo indicariam possíveis destinos alternativos.

O roteamento é resolvido utilizando o Principal mais distante encontrado nesse grafo. O grafo provê um mecanismo alternativo de entrega que é implementado pelo protocolo XIP (eXpressive Internet Protocol) (HAN et al., 2012) que opera em cada nó da rota de acordo com o tipo do Principal destinatário.

O primeiro protótipo da arquitetura XIA utiliza um roteador baseado no Click em plataforma Linux. O grupo responsável pela pesquisa fará a implementação do componente responsável pelo encaminhamento de pacotes. A expectativa é que a implementação baseada em OpenFlow seja mais rápida que a atual. Além disso, essa nova implementação permitirá a execução de experimentos em escalas mais amplas, realizando avaliações mais realistas 
utilizando a GENI, bem como permitirá a implantação da arquitetura utilizando switches comerciais (STEENKISTE, 2011).

\subsubsection{Análise Comparativa}

Há diversos aspectos que norteiam as pesquisas de arquiteturas clean slate para Internet do Futuro. Esta seção apresenta uma análise de trabalhos correlatos. Como referência foram utilizadas duas, entre as cinco iniciativas, que são apoiadas pelo programa NSF FIA (FOUNDATION, 2010). Essas iniciativas foram introduzidas nas seções 2.3.2.1 e 2.3.2.2. Na corrente seção estabelece-se um quadro comparativos das arquiteturas relativamente aos seguintes critérios:

a) Identificação

Identificação (muito frequentemente referenciada como Naming) é uma propriedade fundamental para uma arquitetura, pois permite identificar uma entidade de modo não ambíguo e independentemente da topologia. A separação entre Identificação e Endereço permite que uma entidade seja identificada de modo horizontal (independentemente da localização), facilitando a mobilidade da entidade.

b) Endereçamento

Endereço é um aspecto da localização da entidade que lhe permitirá ser o destinho das comunicações, bem como, permitirá que seja facilmente localizada num ambiente distribuído.

c) Mapeamento entre Identificação e Endereço

Esta capacidade permite também que uma Identificação (um nome) seja mapeado em um endereço correntemente associado a uma entidade. 
Esta capacidade permite que uma entidade possa se movimentar entre ambientes com diferentes tecnologias de acesso, sejam por meio guiados ou não guiados.

d) Nova Pilha de Protocolos

Embora o foco desta comparação sejam arquiteturas clean slate, este critério se faz necessário para atestar (ou não) a criação de uma nova arquitetura.

e) Entrega Multipath

Esta propriedade permite que uma primitiva seja entregue em diversos destinos, ao longo de diversos caminhos (paths), por meio de algoritmos semelhantes a ALM.

f) Endereçamento Multicast

Esta propriedade permite que um conjunto de entidades seja endereçado uma única vez (univocamente), permitindo que o envio de uma primitiva seja suficiente para atingir as entidades destinatárias.

g) Endereçamento Unicast

Esta propriedade permite que uma entidade seja endereçada explicitamente (biunivocamente), permitindo uma relação um-para-um na troca de primitivas entre entidades pares.

h) Roteamento Adaptativo

Esta capacidade permite que duas ou mais entidades se comuniquem por caminhos (rotas) que podem ser alterados durante uma sessão de comunicação. Além dos cenários óbvios, inclui-se aqui o cenário com mobilidade. 
i) Uso de Informação de Roteamento

Esta propriedade permite identificar de que forma a decisão sobre a rota a seguir será operacionalizada nas trocas de primitivas entre duas ou mais entidades pares.

j) Mobilidade

Esta propriedade serve para comparar se a arquitetura permite mobilidade durante uma sessão de comunicação entre duas ou mais entidades pares.

A Tabela 3 apresenta o quadro comparativo entre as arquiteturas XIA e MobilityFirst. Pela mesma é possível perceber que em ambas as arquiteturas existe como premissa uma nova pilha de protocolos e, além disso, uma nova abordagem para endereçamento, não baseada em IP. Outro aspecto que pode ser destacado é a separação clara entre identificação e endereço.Finalmente, pode-se perceber que em ambas propostas existe um suporte ao Multicast, corroborando a noção de sua importância.

Tabela 3: Comparação entre Arquiteturas de Internet do Futuro XIA e MobilityFirst.

\begin{tabular}{|c|c|c|}
\hline \multirow{2}{*}{ Critério } & \multicolumn{2}{|c|}{ Arquiteturas de Internet do Futuro } \\
\hline & XIA & Mobility First \\
\hline Identificação & Principal (XID) & GUID \\
\hline Endereço & DAG & Endereço Rede \\
\hline Mapeamento entre Identificação e Endereço & XARP & GNRS \\
\hline Nova Pilha de Protocols & Sim & Sim \\
\hline Entrega multipath & Sim & $\operatorname{Sim}$ \\
\hline Multicast & $\begin{array}{l}\text { Baseado em um } \\
\text { tipo espefício de } \\
\text { Principal }\end{array}$ & $\begin{array}{l}\text { Baseado em } \\
\text { Grupo Multicast }\end{array}$ \\
\hline Unicast & Baseado no XID & $\begin{array}{l}\text { Baseado em } \\
\text { GUID }\end{array}$ \\
\hline Roteamento Adaptativo & XIP & GSTAR \\
\hline Uso de Informação de Roteamento & Hop-a-Hop & Hop-a-Hop \\
\hline Mobilidade & Nativo & Nativo \\
\hline
\end{tabular}




\section{ESPECIFICAÇÃO DA ARQUITETURA ETARCH}

O conceito de endereçamento Multicast foi introduzido desde as primeiras arquiteturas de rede e, como mostrado no capítulo anterior, está presente tanto nas camadas inferiores (até a camada de rede do Modelo de Referência OSI), quanto nas camadas superiores, sendo particularmente explorado na camada de aplicação (ALM).

Todavia, devido a aspectos tais como granularidade e dinamismo, as capacidades de Multicast das camadas inferiores são praticamente não utilizadas. Nos dias atuais, ALM é a saída para diminuir a replicação de dados, sendo que é possível notar em 2.1.3 que restam ainda significativa retransmissão (store-and-forward) e replicação de primitivas.

Requisitos tais como mobilidade, QoS e QoE não são suportados pelas arquiteturas de redes atuais, notadamente a arquitetura Internet, mesmo se considerar o protocolo IPv6, motivo pelo qual aplicações como VoIP, Video Conferência e VoD não funcionam adequadamente.

Este capítulo apresenta a Arquitetura ETArch (Entity Title Architecture) cujo principal objetivo é aproximar semanticamente a camada de aplicação e as camadas inferiores. A aproximação semântica permite oferecer mecanismos para agregação de tráfego Multicast e facilita expressar os requisitos das aplicações de tal modo que eles permeiem a arquitetura em todas as suas 
camadas.

Além disso, é apresentada a abordagem utilizada na identificação e no endereçamento das entidades, bem como os protocolos envolvidos e os aspectos relacionados à arquitetura proposta tais como modos de transmissão, roteamento e mobilidade.

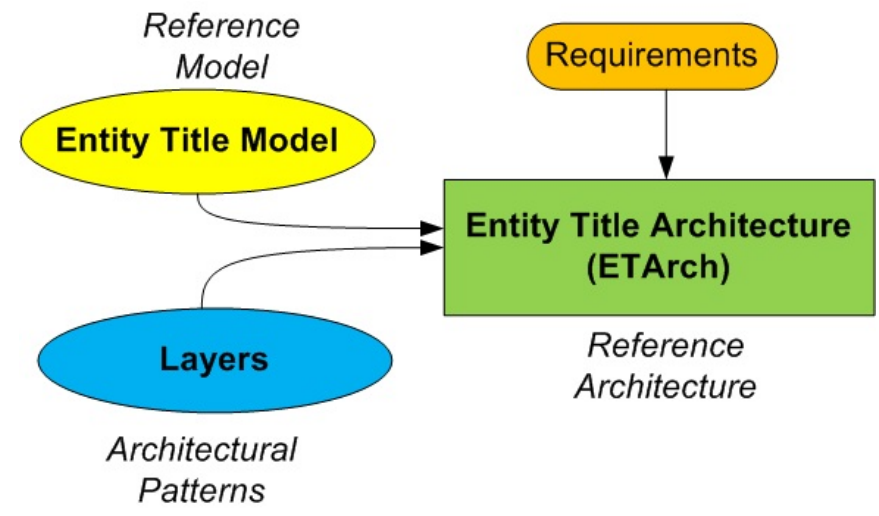

Figura 12: Processo para definição da ETArch (Entity Title Archicture) (BASS; CLEMENTS; KAZMAN, 2003).

O processo de definição da arquitetura parte de uma filosofia clean slate, cuja premissa é uma nova arquitetura. Este processo se inicia com um modelo de referência (BASS; CLEMENTS; KAZMAN, 2003), neste caso o Entity Title Model (PEREIRA et al., 2011), e padrões arquiteturais (BUSCHMANN et al., 1996) (SCHMIDT et al., 2000) adequados ao domínio em questão, e leva em conta os requisitos propostos para esta arquitetura, conforme mostrado na Figura 12. A contribuição deste trabalho é uma arquitetura de referência: a arquitetura ETArch.

A arquitetura ETArch pode ser considerada uma realização do Entity Title Model cujos conceitos são a base para este trabalho. O Entity Title Model é uma visão sobre como entidades podem descrever de forma semântica seus requisitos e capacidades necessárias para suas comunicações.

ETArch se diferencia das arquiteturas tradicionais, incluindo-se entre elas 
a arquitetura Internet, pois a distância entre as aplicações e as camadas inferiores (notadamente as camadas Física e de Enlace) não é fixa e, também, não apresenta um "gap" que dificulta a passagem dos requisitos de QoS/QoE das aplicações às camadas inferiores. Por exemplo, na arquitetura Internet, a interface Socket não permite a uma aplicação expressar requisitos de vazão ou de Jitter.

\subsection{Aspectos Conceituais da ETArch}

Esta seção descreve os principais conceitos propostos pelo Entity Title Model e seus usos pela ETArch. Além dos conceitos, são descritos os principais componentes e as respectivas relações com a arquitetura.

\subsubsection{Entidade}

Entidade (Entity) é o elemento fundamental da ETArch. Uma Entidade possui necessidades de comunicação e capacidades que podem ser entendidas semanticamente por todas as camadas existentes na arquitetura. Alguns exemplos de entidades podem ser: uma aplicação, um usuário, um sensor, um smartphone, um host, uma coisa (Thing), etc.

Uma Entidade especifica entes da arquitetura ETArch que possuem um conjunto de requisitos, uma lista de capacidades, comportamentos e interface para relacionamento.

Uma Entidade possui ao menos um Título (Title - ver seção 3.1.2), por meio do qual ela pode ser unicamente identificada, e uma localização, denominada Ponto de Conexão (PoA - Point of Attachment). Esta separação é importante, pois a localização de uma entidade pode variar ao longo do tempo (mobilidade). Um título pode ser visto como uma credencial que pode ser 
usada para relacionar aspectos de segurança (UNION, 1991). Exemplos de PoA podem ser switches, roteadores, Access Points, etc.

Entidades podem manter relações com outras entidades e, através dessas relações, podem herdar propriedades, exceto título. Por exemplo, uma aplicação pode estar ligada a um host e um sensor pode estar vinculado a um smartphone.

A definição de Entidade permite que a arquitetura ETArch possa satisfazer diferentes conjuntos de requisitos, propostos por diferentes visões da Internet do Futuro centradas por exemplo em Serviços (Service-Centric) (MARTINEZ et al., 2012), Usuários (User-Centric) (BALADRóN et al., 2009), Conteúdo (Content-Centric) (JACOBSON et al., 2012) e Dispositivos em geral (IoT - Internet of Things) (ATZORI; IERA; MORABITO, 2010), enquanto pode suportar a visão atual baseadas em servidores (host).

O princípio de projeto associado a esta abordagem é a flexilidade, que permite lidar com uma grande diversidade de requisitos (atualmente propostos para as redes de computadores) e, além disso, ser genérica o suficiente, com capacidade evolutiva, para abraçar futuros requisitos.

\subsubsection{Título}

O Título (Title) é uma identificação de entidade, única, não ambígua e independente da topologia. Uma entidade tem uma relação unívoca (um para muitos) com Títulos. O uso do termo Título tem origem na definição (simplificada) de (n)-Entity-Title apresentada em (UNION, 1996).

Um conjunto de Títulos são agrupados em um Namespace. Namespace é um espaço de identificação discreto, único, e é responsável por adicionar um significado inicial na relação entre Título e Entidade. Um Título é único 
e não ambíguo dentro de um Namespace, que também deve ser único. Alguns exemplos de entidades indentificáveis em um Namespace e, portanto, passíveis de serem tituladas são: host, sensor, phone, conteúdo, etc.

Um Título é definido por uma tupla, cuja especificação é Namespace::Identificacao-entidade. Por simplicidade, no decorrer deste texto Namespace será omitido.

Considerando que Namespaces devem ser únicos, então é necessária uma organização, por exemplo, como a ICANN (Internet Corporation for Assigned Names and Numbers), que seja responsável por garantir a unicidade dos 'Nomes'. Os Títulos, por sua vez, podem ser geridos por serviços de nomes que seriam providos pelo servidor de Namespace. O escopo desses serviços poderia ser local ou global.

Quando gerenciado localmente, o Título deve ser seguido de um nome de domínio, garantindo assim sua unicidade global. Como o Título é único, qualquer entidade pode ser corretamente identificada, independentemente do endereço do PoA. Este endereçamento é utilizado pelo modo de encaminhamento objeto deste trabalho. A unicidade e independência de localização favorece a mobilidade dessas entidades. O título tem um papel central a fim de proporcionar o endereçamento horizontal (PEREIRA; KOFUJI; ROSA, 2010) das entidades.

Um Título associado a entidades na camada de aplicação geralmente é expresso por meio de um formato legível, tais como caracteres Unicode. Por sua vez, o título associado a entidades na camada de rede pode, por exemplo, ser expresso por meio de uma cadeia de bits. 


\subsubsection{Workspace}

Para usuários em geral, os acessos à rede não são feitos em busca de uma máquina ou um servidor. Em geral, busca-se por informações, serviços, conteúdos, vídeos, etc. Observe-se que essas entidades (informação, serviço, conteúdo, vídeos, etc) carregam-se de semântica pertinente a usuários. O endereçamento de tais tipos de entidades se dá ao nível de Aplicação.

Do ponto de vista conceitual, as comunicações entre entidades parceiras (peer entities), independentemente das camadas às quais pertençam, se dão através de canais. Observe-se que entidades não se comunicam diretamente, mas se comunicam por meio do canal, isto é, enviam suas primitivas ao canal, que se incumbe de levá-las até seu(s) destino(s).

Os canais estabelecem um tipo de relacionamento um-para-um entre as entidades parceiras. Se mais de duas entidades desejam comungar suas comunicações, então há que se lançar mão da estratégia introduzida por ALM (ver 2.1.3). Contudo, a estratégia ALM de endereçamento é pouco flexível e não elimina a necessidade de retransmissão.

A arquitetura ETArch introduziu um conceito de comunicação multi-end que passou a ser denominado de Workspace. Conceitualmente, Workspace é um barramento lógico, independente da topologia, ao qual entidades podem se ligar (Attach) para participar de um domínio de comunicação. Um Workspace pode ser constituído por redes cabeadas (Wireline) e redes sem fio (Wireless), sendo que entidades conectadas a redes cabeadas podem interagir com entidades conectadas em redes sem fio transparentemente.

Workspace se diferencia de ALM, principalmente, nos seguintes aspectos: naturalmente multicast, não há replicação de primitivas; dinâmico, permite que entidades se [des]liguem facilmente do domínio de comunicação; 
transparente à mobilidade, mantêm transmissão de stream sem interrupção; e, transparente ao meio físico, permitem estabelecer barramento lógico envolvendo redes cabeadas e redes sem fio.

Um Workspace é identificado por seu título e pode ser distribuído através de um ou mais elementos de rede (NE), cabeados ou sem fio, e possuem as seguintes propriedades:

- Título: elemento chave, identifica univocamente um Workspace;

- Lista de NE: enumera Títulos de elementos de rede que fazem parte do Workspace;

- Lista de Capacidades: enumera as capacidades que po dem ser oferecidas pelo Workspace às entidades. Alguns exemplos: parâmetros de QoS associados à comunicação, confidencialidade de dados, entre outras;

- Lista de Requisitos: enumera os requisitos que as entidades que desejam anexar-se um determinado Workspace devem suportar. São semeIhantes às Capacidades, porém neste caso representam requisitos que devem ser satisfeitos por estas entidades;

- Visibilidade: define se um Workspace é Público ou Privado.

- Nível: número natural que define o âmbito do domínio do Workspace sendo que 'zero' permite visibilidade mundial.

Um workspace é criado quando uma entidade produz algo que pode ser consumido por outra(s) entidade(s), tal como vídeo-conferência, compartilhamento de arquivos, etc. A iniciação, manutenção e encerramento de um workspace é controlado por um sistema de títulos denominado de DTS (Domain Title 
Service). O DTS é constituído de agentes denominados DTSA (DTS Agent) que são introduzidos em 3.1.4.

A criação de workspaces é feita a partir de requisições que entidades enviam ao DTS. A requisição informa os requisitos que devem ser suportados pelo Workspace, bem como as capacidades oferecidas e demais propriedades relativas a essa instância de comunicação. As seções 3.5.1 e 3.5.2 apresentam os serviços dos protocolos da Arquitetura ETArch que são responsáveis pela iniciação, manutenção e encerramento de workspaces.

Uma vez criado, um workspace está apto a ser compartilhado por entidade(s) que queira $(m)$ fazer parte deste domínio de comunicação. Toda entidade, que atenda às propriedades do workspace, pode se ligar (attach) ao workspace e, neste caso, o DTS é responsável por reconfigurar os NEs a fim de incluir a entidade requisitante ao workspace.

Se se tratar de uma entidade móvel, quando ela se movimenta de um PoA para o outro, o DTS reconfigura o workspace para suportar a mobilidade das entidades pela rede.

O ponto central da ETArch é que o Título do Workspace é o endereço de destino das primitivas durante as comunicações, tanto de dados quanto de controle, e não os Títulos das Entidades parceiras, isto é, uma entidade envia primitivas para o Workspace e as entidades parceiras recebem-nas desse Workspace.

Portanto, o foco deste trabalho é a capacidade nativa do Workspace de suportar endereçamento multicast mais eficientemente do que as abordagens utilizadas no âmbito da camada de rede (IP Multicast) ou de aplicação (ALM).

A Figura 13 representa o Workspace e demais componentes da arquitetura que suportam seu funcionamento. 


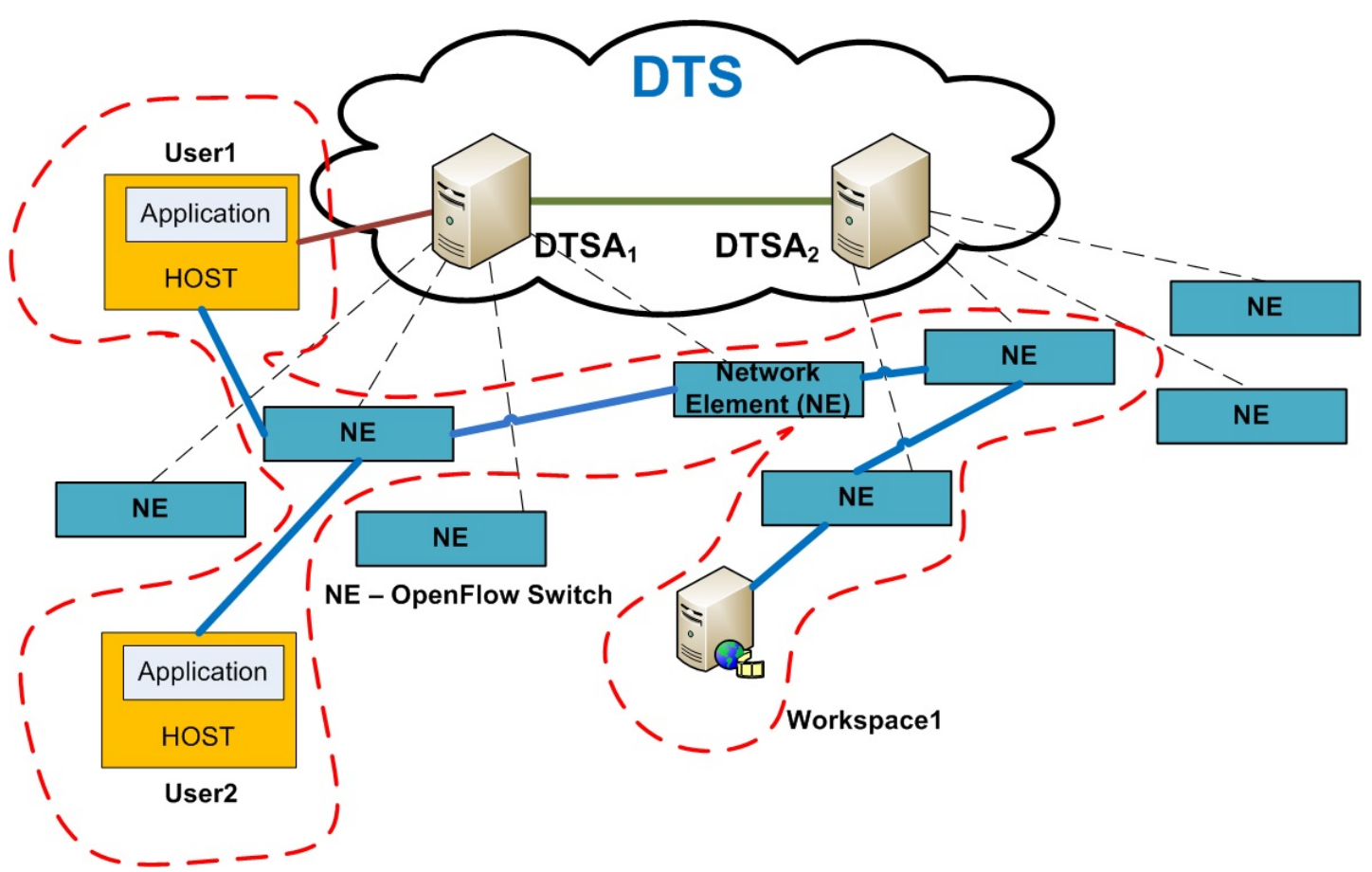

Figura 13: Principais Componentes da Arquitetura - DTS, DTSA, Entity, Title e o Workspace.

\subsubsection{Workspace de Controle}

Workspaces de Controle existem para gerenciar, manter e controlar o ambiente distribuído do DTS, bem como gerenciar o ciclo de vida de entidades (registro, controle de acesso e operação). Workspaces de Controle diferem de Workspaces de Dados em três aspectos principais:

- Natureza: transmitem apenas primitivas dos protocolos de controle, não servindo para a transferência de dados genéricos das aplicações;

- Criação: são criados a priori, durante a configuração do ambiente, e praticamente não mudam ao longo do tempo; e,

- Entidades: ligam-se a este tipo de workspace apenas entidades dos tipos DTSA, MDTSA e NE. 
Como agentes do DTS (DTSAs) também são entidades, então suas comunicações são feitas através de workspaces. Ressalte-se que as primitivas trocadas entre os DTSAs visam o controle do ambiente distribuído e não servem a transferências de dados de aplicações. Todo Workspace de Controle tem ao menos um DTSA responsável pelas resoluções de Títulos naquele espaço denominado MDTSA (Master DTSA).

Workspaces de Controle são criados durante a inicialização de cada DTSA. Para o momento deste trabalho, os Workspaces de Controle são criados de forma manual, porém futuramente um serviço poderá ser construído para oferecer as funcionalidades de controle do DTS.

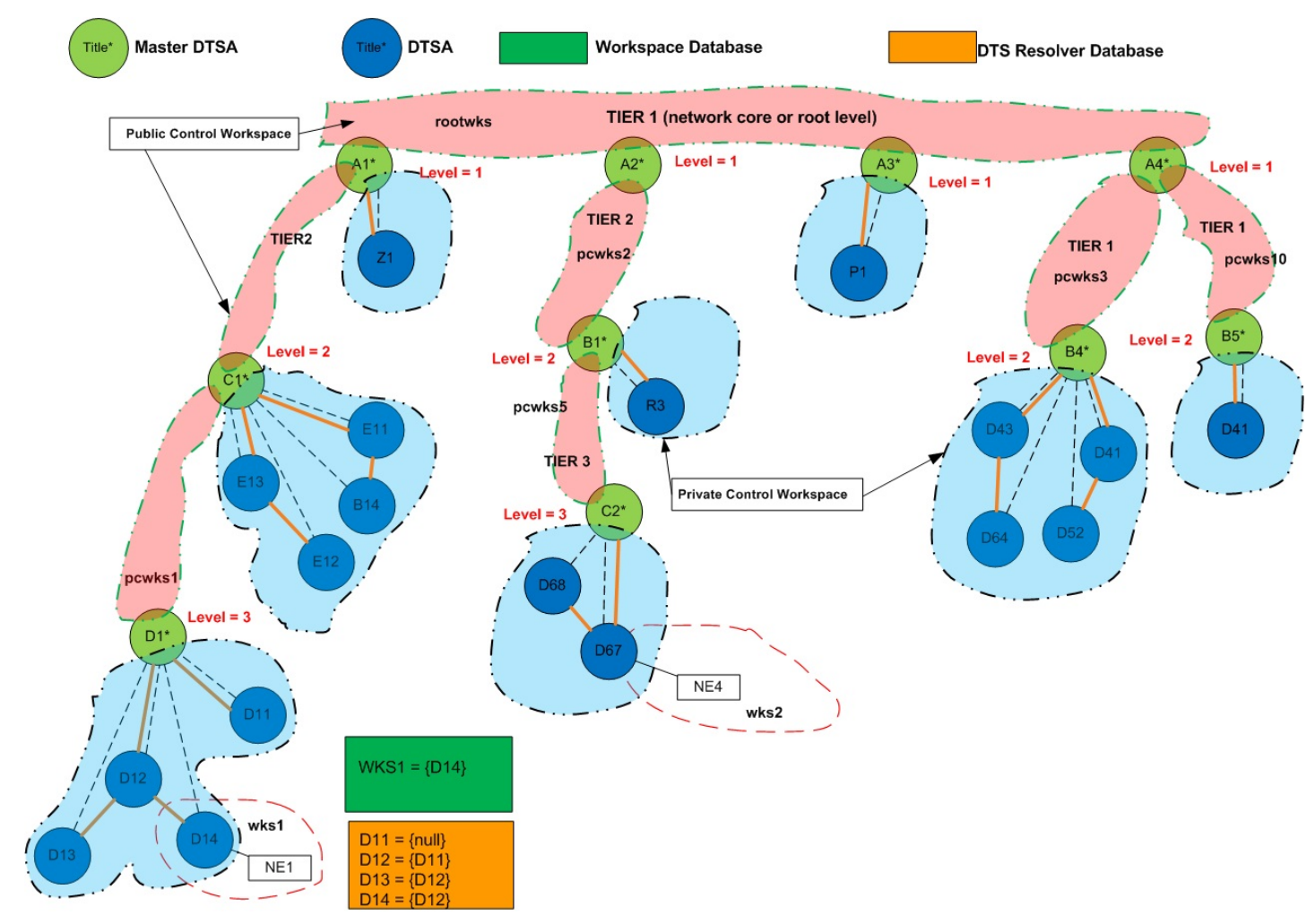

Figura 14: Workspaces Públicos e Privados no DTS.

Os Workspaces de Controle podem ser Públicos (Public Control Workspaces) ou Privados (Private Control Workspaces). Workspaces de Controle Privados interconectam um ou mais agentes do tipo DTSA e um agente do tipo MDTSA, que os controla (os DTSAs). Deste modo, as comunicações 
entre o(s) DTSA(s) e o MDTSA são suportadas por este tipo de workspace privado.

Workspaces de Controle Públicos interconectam (dois ou mais) agentes exclusivamente do tipo MDTSA. Neste contexto, a propriedade Level de um MDTSA e dos DTSAs a ele associados, por sua vez, também pode ser definida como o número de Workspaces de Controle Públicos entre o referido MDTSA e a raiz, isto é, o núcleo da rede. A Figura 14 representa Workspaces de Controle Públicos (rosa) e Privados (azul) que constituem o DTS.

\subsubsection{Workspaces de Dados}

Workspaces de Dados (Data Workspaces) existem para suportar a troca de primitivas de envio de dados entre Aplicações (Entidades) de propósito geral tais como Video Conference, HDTV, entre outras. Os Workspaces de Dados são criados por entidades que tenham propriedades para tal e que tenham algo a ser consumido em um ambiente distribuído, conforme exibido na Figura 15 a seguir.

Workspaces de Dados são criados, mantidos e encerrados dinamicamente através de serviços do Plano de Controle (composto por Workspaces de Controle), sendo que durante seus ciclos de vida estão disponíveis para receber a anexação de Entidades que queiram compartilhar os Workspaces de Dados.

Considerando que dispositivos móveis podem ser deslocar ao longo de Workspaces de Dados, então o Plano de Controle tem de manter as configurações dos NEs de tal modo que esses elementos reflitam as necessidades de conectividade desses tipos de entidades. 


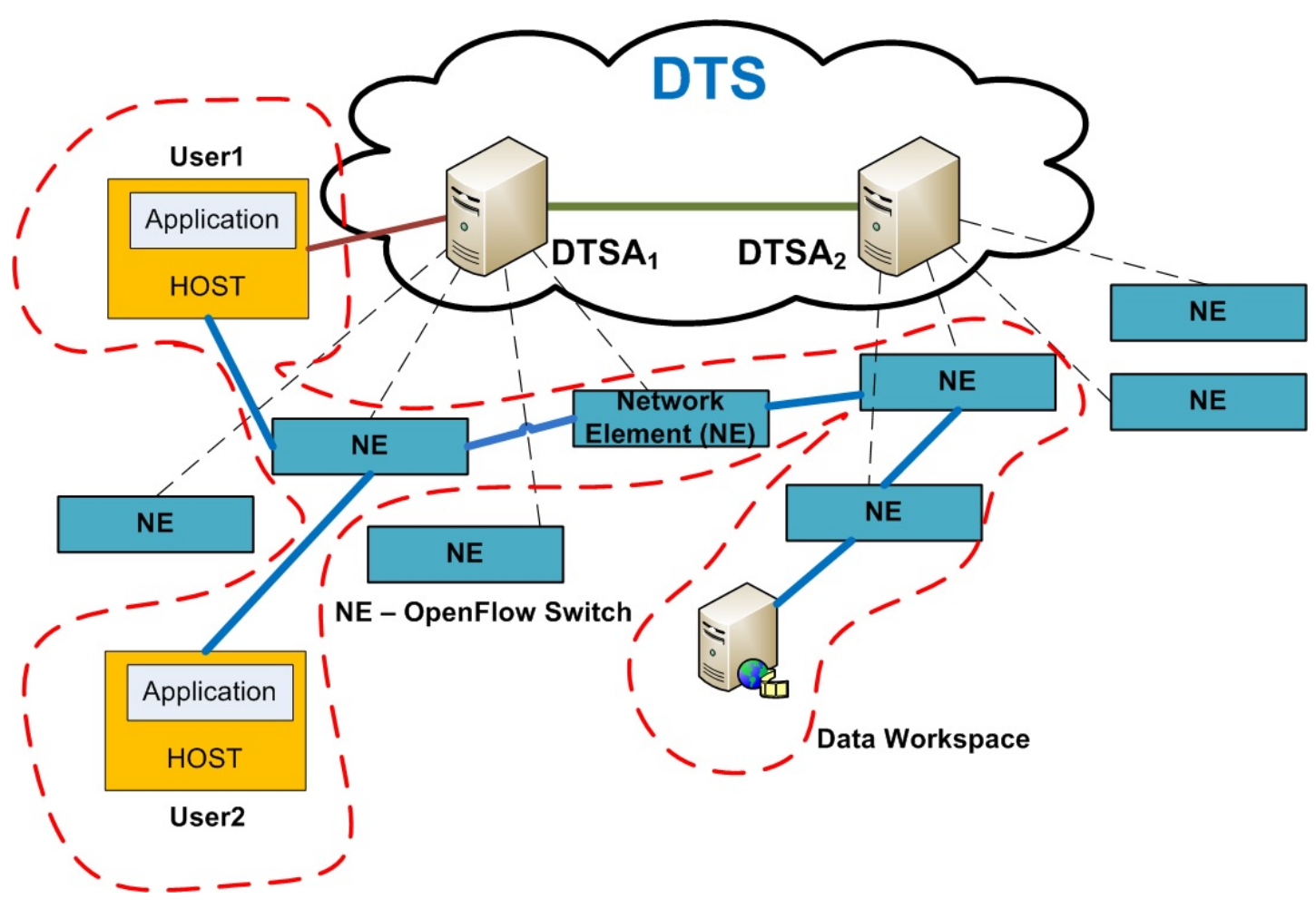

Figura 15: Workspace de Dados.

\subsubsection{Serviço de Domínio de Títulos}

O Serviço de Domínio de Títulos (DTS - Domain Title Service) trata todos os aspectos ligados à resolução de Títulos, ao gerenciamento do ciclo de vida das Entidades no ambiente distribuído e, particularmente, manutenção da relação entre Entidades (3.1.1) e Títulos (3.1.2).

O DTS mantém a base de conhecimentos sobre os detalhes do ambiente (Workspaces de Controle, Workspaces de Dados, Entidades, Títulos e Elementos de Rede) e, portanto, é capaz de monitorar e controlar os requisitos de comunicação de Entidades ao longo do tempo.

O DTS é composto por um conjunto de um ou mais agentes denominados DTSA (Domain Title Service Agent), como representado na Figura 16 a seguir, os quais são responsáveis por implementar aspectos da gestão de uma localidade (sub conjunto) do domínio. A interconexão dos DTSAs é feita 
por Elementos de Redes (NE - Network Element), por exemplo switches, que podem ser exclusivos para o Plano de Controle (Control Plane) ou compartiIhados com o Plano de Dados (Data Plane).

Da mesma forma, por exemplo, na presença de uma falha de um elemento de rede, o DTS é capaz de realizar a reconfiguração do workspace, a fim de manter a comunicação.

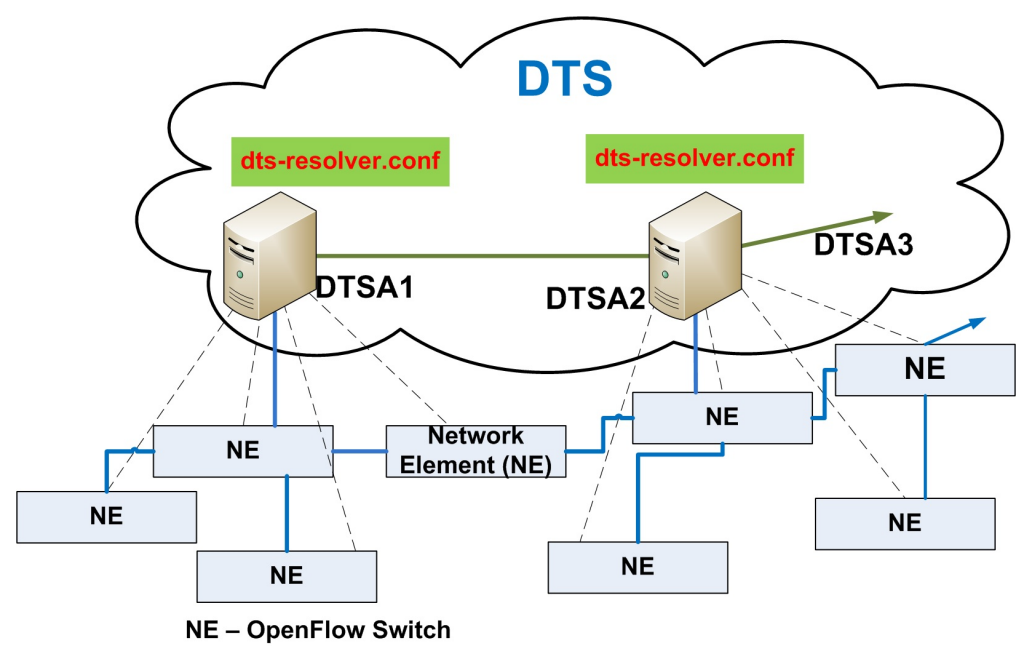

Figura 16: Visão Geral do DTS

Os NEs representados na Figura 16 constituem o plano de dados da arquitetura ETArch e são monitorados/controlados pelo DTS (de fato pelos DTSAs) para que atendam aos requisitos de comunicação das Entidades (Aplicações). O controle/monitoração dos NEs é feita em regime permanente e, portanto, podem modificar seus comportamentos em tempo de execução.

Essa abordagem, proposta inicialmente em (PEREIRA; KOFUJI; ROSA, 2009), encaixa-se naturalmente às abstrações de SDN, onde um controlador centralizado é capaz de alterar o comportamento do plano de dados. Deste modo, o conjunto de funcionalidades dos DTSAs é um super conjunto das funcionalidades disponíveis no controlador introduzido pelo OpenFlow (MCKEOWN et al., 2008) $)^{1}$.

\footnotetext{
${ }^{1}$ OpenFlow é uma materialização do SDN
} 
O DTSA gerencia o ciclo de vida de Entidades, desde seu registro, durante os contextos de comunicação, até a finalização de suas atividades. Além de informações sobre Entidades, um DTSA mantém informações sobre os NEs (requisitos, restrições, capacidades e grafo de interconexão).

Responsável por lidar com aspectos do sistema - tais como identificação, endereçamento, transferência de dados, roteamento, mobilidade, QoS, controle de tráfego e de sessão, entre outros - cada DTSA tem controle sobre uma parte da rede e para realizar suas funções e pode ter uma relação de confiança (federation) com outros DTSAs. Se um DTSA não possui informações sobre um Título, então ele pode consultar outros DTSAs, com os quais possua relação de confiança, que são denominados Resolvers (identificados por seus títulos).

Por exemplo, como mostrado na Figura 16, cada DTSA possui um arquivo de configuração, denominado dts-resolver.conf, que contém uma lista de um ou mais títulos, na qual cada Título identifica um Workspace de controle.

Seguindo a filosofia SDN, os comportamentos dos NEs são controlados externamente pelos agentes DTSA numa relação unívoca, sendo que um DTSA pode controlar uma lista de um ou mais NEs e um NE é controlado por apenas um DTSA. Para uma rede de pequeno porte, com um número pequeno de NEs, pode ser que um DTSA seja suficiente para controlar seus comportamentos. Uma rede de maior porte pode necessitar de mais de um DTSA.

Um conjunto de DTSAs pode ser especificado para representar um domínio, por exemplo um campus, uma região geográfica, etc, sendo que um DTSA, definido para representar este conjunto, com responsabilidades adicionais, denominado Master DTSA (MDTSA). O MDTSA é a interface de contato entre dois ou mais domínios cujos Workspaces de Controle podem ser Públicos ou Privados. Se um domínio é pequeno o suficiente para demandar 
apenas um DTSA, então este fará também o papel de MDTSA. O MDTSA possui informações sobre o grafo de interconexão de DTSAs do domínio.

Assim como no caso de arquiteturas tradicionais, haverá domínios especializados em oferecer interconexão (SP - Service Providers), como, por exemplo, é o caso dos atuais ISPs (Internet Service Provider). Um MDTSA pode utilizar diferentes SPs para interconexão com o mundo exterior, possibilitando uma relação Multihomming. Deste modo, um MDTSA de um domínio específico (por exemplo, uma empresa) interfaciará com o MDTSA de um SP através de um Workspace de Controle Público.

MDTSAs, e DTSAs, possuem a propriedade Level, que indica sua distância em relação à raiz da árvore de MDTSAs (Root Level), cujo Level é zero. A estrutura de árvore pode apresentar $n$ níveis, sendo que cada MDTSA implementa a interface entre dois diferentes níveis. DTSAs de um determinado nível possuem, ao menos, um MDTSA nesse nível. Exceção feita aos MDTSAs que estão na interface Root Level, que contam apenas com outros MDTSAs em seu conjunto de Resolvers. Na Figura 17 a seguir, a linha em vermelho entre dois MDTSAs é uma ligação lógica, sendo que as linhas em laranja entre DTSAs e MDTSAs são ligações físicas.

A Figura 17 apresenta uma árvore hierárquica, de três níveis do DTS, que pode suportar uma visão mundial da rede. O nível local representa domínios locais, sendo que cada um contém ao menos um MDTSA. O nível 2 representa SPs que fornecem acesso às redes de nível 1, mas não possuem uma cobertura mundial e necessitam contratar acesso de outros SPs para chegar a outras regiões de uma rede mundial.

O nível 1 é constituído por SPs que podem acessar todos os ramos da rede mundial. Estes SPs de nível 1, também denominados Tier 1, representam o Root Level, o núcleo (core) da rede mundial. Os MDTSAs que constituem o 


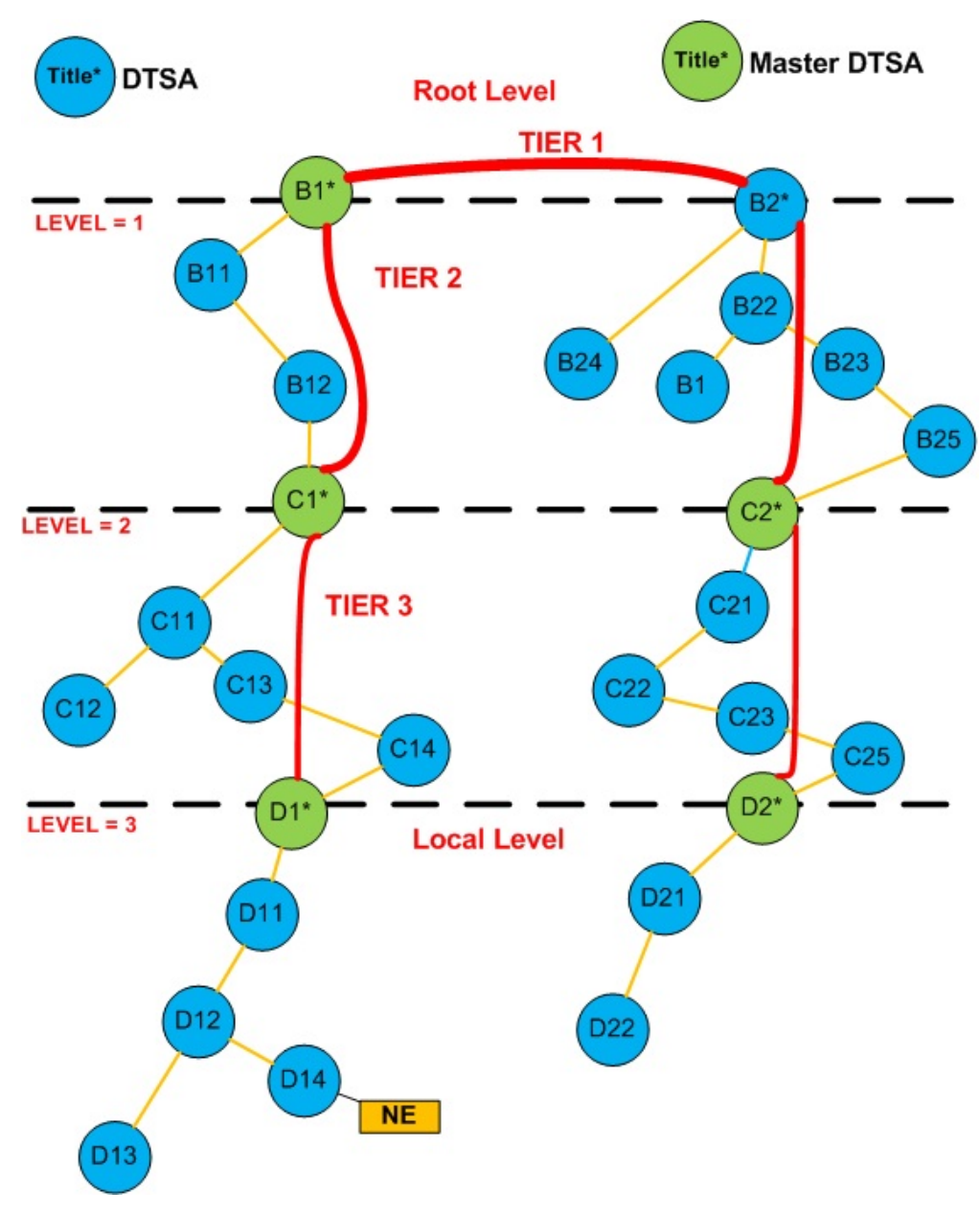

Figura 17: DTS como uma Rede Mundial em Três Níveis.

núcleo da rede, e não possuem Resolvers em um nível superior, compartilham um Workspace de Controle que Ihes permite trocar primitivas numa relação de Peering. Essa organização segue a abordagem de camadas (Tier) 1, 2 e 3 da Internet atual.

Para suportar as responsabilidades adicionais, o MDTSA possui dois componentes de resolução, sendo: 1) DTS Resolver Database; e, Workspace Database. O DTS Resolver Database de um MDTSA mantém informações sobre todos os Resolvers de DTSAs sob seu controle. O Workspace Database de um MDTSA possui informações sobre os Workspaces associados a cada DTSA sob seu controle. A Figura 18 a seguir ilustra os componentes de um nível $i$ genérico do DTS. 


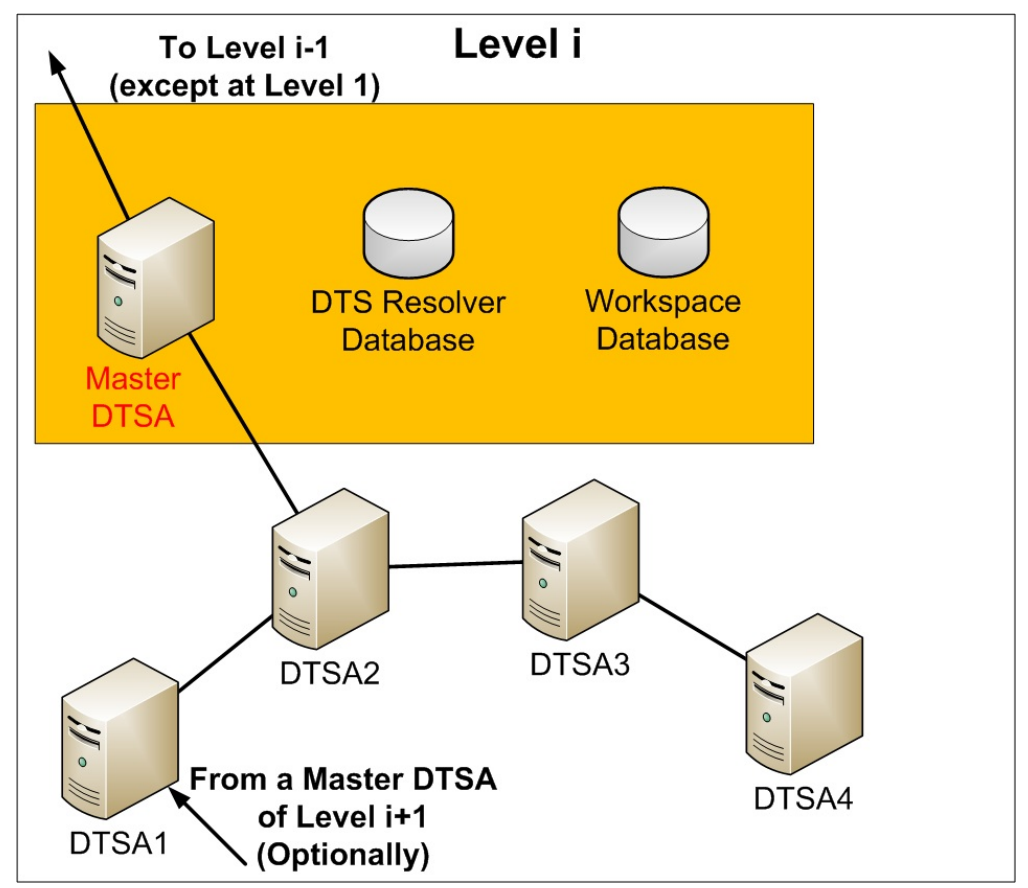

Figura 18: Componentes de um Nível Genérico do DTS.

Em determinado nível, o Workspace Database possui informações sobre os workspaces existentes nesse nível. Cada entrada nessa base de dados possui um ou mais DTSAs desse nível onde um determinado workspace pode ser alcançável naquele momento. Na figura 19 a seguir, os workspaces WKS1 e WKS2 são acessíveis por qualquer DTSA do nível 3. Se um dado workspace não é conhecido pelo MDTSA D1, por exemplo, a consulta será encaminhada para o MDTSA $C 1$.

Para a resolução de títulos, o DTS Resolver Database mantém informações sobre o grafo de interconexão de DTSAs sob controle do MDTSA. Toda vez que é inicializado, cada DTSA requisita um serviço para seu registro no MDTSA. Durante o registro, o MDTSA armazena no DTS Resolver Database informações relacionadas ao DTSA a fim de criar o grafo de DTSAs interconectados sob o controle de um específico MDTSA.

Por exemplo, uma representação possível desse grafo seria um dicionário, onde cada entrada conteria o Título de um DTSA e uma lista de Títulos 


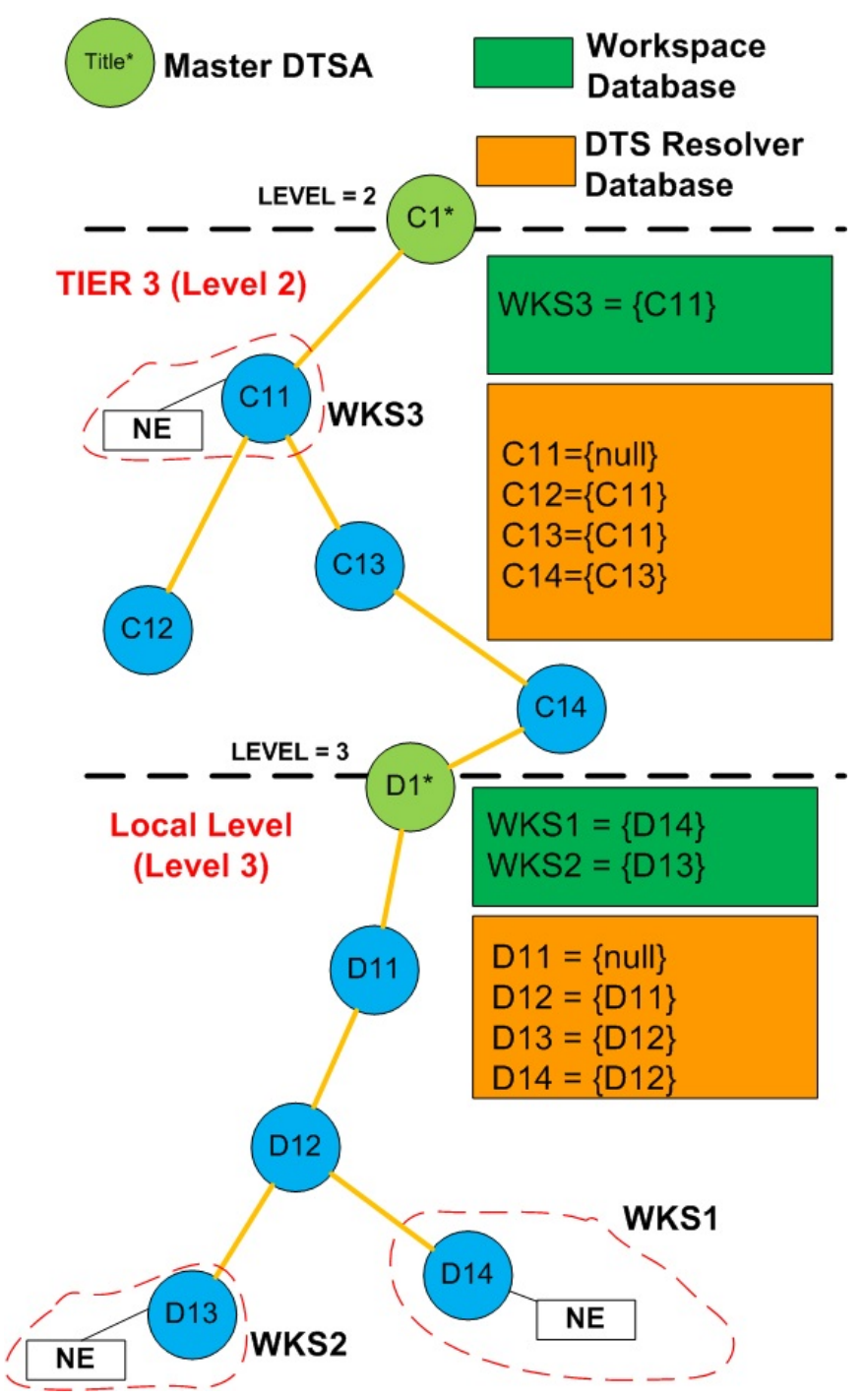

Figura 19: MDTSA - Exemplos de valores no Workspace Database e DTS Resolver Database.

de DTSAs diretamente conectados a ele. No caso de um DSTA estar diretamente conectado ao MDTSA, então essa lista não conteria elementos, apenas uma entrada indicando null, conforme ilustrado na Figura 19. O número de entradas no DTS Resolver Database de um MDTSA será igual ao número de DTSAs sob seu controle. Uma entrada no dicionário será criada durante o processo de registro de um DTSA, e se for alterado ao longo da operação, então o DTS Resolver Database deve ser atualizado. 


\subsection{Camadas ETArch}

Redes de computadores são essencialmente baseadas em protocolos que suportam a comunicação entre processos pares (DAY, 2008). Toda arquitetura de rede deve levar em conta uma miríade de protocolos utilizados pelas aplicações atuais. Uma nova arquitetura tem de considerá-los para ser passível de adoção. Da mesma forma, a camada física oferece diversos protocolos e tecnologias de meios físicos, que devem ser considerados uma vez que são largamente utilizados.

Neste cenário, o padrão arquitetural em camadas (Layer) é adotado como ponto de partida para a especificação da Arquitetura ETArch. Embora utilize uma abordagem clean slate, ETArch se propõe a suportar os protocolos da camada de Aplicação e a utilizar os protocolos da camada Físca existentes, levando-se em conta a nomenclatura utilizada pelo modelo de referência OSI (Open Systems Interconnection) (TANENBAUM, 2002).

A arquitetura ETArch define, de cima para baixo, três camadas denominadas: Application, Communication e Link, representadas na Figura 20 a seguir. No geral a arquitetura ETArch reexamina as camadas de Transporte e de Rede da arquitetura Internet e do modelo de referência OSI. Com esta premissa, o foco deste trabalho é basicamente a camada Communication.

A representação não usual da camada Communication tem o objetivo de transmitir a capacidade que a arquitetura ETArch tem de se adaptar a requisitos de Entidades de Aplicação. Dependendo do contexto de comunicação, pode ser que o overhead da camada Communication seja mínimo, representado pela porção delgada (lado esquerdo da Figura 20) a seguir, ou até mesmo inexistente.

Por exemplo, imagine que duas Entidades de Aplicação estejam se co- 

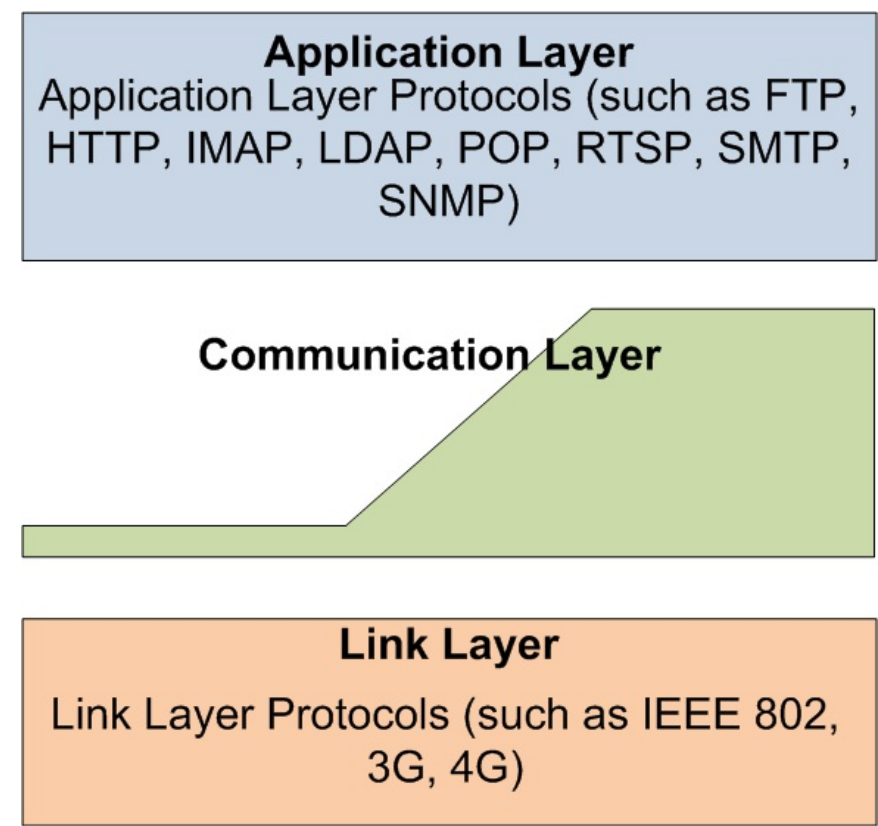

Figura 20: Camadas definidas pela ETArch.

municando em uma rede local através de serviços não orientados a conexão (ConnectionLess Services), que não ofereçam garantia de entrega, então elas precisariam apenas do enlace lógico.

No outro extremo, pode ser que o contexto de comunicação necessite de funcionalidades que imponham overhead máximo da camada Communication, representado pelo lado direito da Figura 20.

Suponha-se, por exemplo, que duas ou mais Entidades de Aplicação estejam distribuídas em diversos domínios (redes) e se comuniquem através de serviços orientados a conexão (Connection Oriented Services), com garantia de entrega e requisitos de segurança (confidencialidade, integridade, não repúdio, auntenticidade, entre outros), então elas precisariam de roteamento, serviços confirmados, criptografia, certificados, etc.

A camada Communication recebe essa denominação por sua identidade filosófica com o termo Communicare em Latim. Comunicação significa pôr em comum, conviver. Esse "pôr em comum" implica que transmissor e receptor 
estejam dentro do mesmo contexto, caso contrário não haverá compreensão. Assim, comunicação deve levar consigo a ideia de compreensão. Na arquitetura ETArch há o compromisso da compreensão nos dois planos - de Controle e de Dados.

No plano de Controle, o compromisso é fazer com que a configuração de Workspaces de Dados atendam aos requisitos de QoS ou QoE definidos por Entidades de Aplicação. Observe-se que neste plano há a necessidade de interações com NEs e, então, a filosofia SDN vem ao encontro do que a ETArch se propõe. O DTS (de facto DTSAs), através de Workspaces de Controle, tem a responsabilidade de compreender os requisitos de Entidades de Aplicação e traduzí-los para a configuração dos NEs na criação ou extensão de Workspaces de Dados.

Dessa forma as aplicações correntes ${ }^{2}$, que necessitem de utilizar tais abstrações, devem ser atualizadas a fim explorar os novos recursos oferecidos pela arquitetura. Todavia, as entidades da camada Application são suportadas pela arquitetura ETArch, pois a adoção da interface Socket oferece serviços amplamente conhecidos e, portanto, as mensagens da camada Application são encapsuladas em primitivas a serem manipuladas pela camada Communication.

Em relação à camada Link subjacente, a adoção da filosofia SDN permite que a camada Communication utilize interfaces com serviços de enlace padronizados, o que a torna naturalmente compatível com os serviços atualmente oferecidos pelas tecnologias de enlace atuais, como OpenFlow, e emergentes.

O plano de Controle tem a missão de abstrair os detalhes da configuração de NEs, de modo que os requisitos de Entidades de Aplicação sejam perfeita-

\footnotetext{
${ }^{2}$ Entidades de aplicação na linguagem da arquitetura ETArch
} 
mente compreendidos e atendidos na construção de Workspaces de Dados. Desse modo, os serviços de Workspaces de Dados são reduzidos em número, mas com classes de abstração conforme mostra a seção 3.3.

\subsection{Concepção de Serviços na ETArch}

No plano de Dados, como o próprio nome diz, o compromisso é com a transmissão de primitivas de dados sendo que há basicamente três classes de serviços:

- Serviços Confirmados: serviço no qual a entidade requisitante do serviço de dados recebe uma confirmação (Acknowledgement ou simplesmente Ack) para cada requisição3;

- Serviços Não-confirmados: serviço no qual a entidade requisitante do serviço de dados não recebe evidência de que a primitiva foi entregue; e,

- Serviços Piggybacking: serviço no qual a entidade requisitante do serviço de dados, ao mesmo tempo em que requisita um envio de dados aproveita para confirmar o recebimento de dados recebidos por ela anteriormente.

Observe que os Serviços Confirmados (Confirmed Services) servem às entidades que requerem uma evidência de que a primitiva atingiu seu(s) destino(s) e, portanto, são comumente referenciados como serviços confiáveis, enquanto os Serviços Não-confirmados (Unconfirmed Services) são por natureza não confiáveis uma vez que a Entidade requisitante não tem uma evidência de que a primitiva foi entregue. Note-se que o termo "Confiável" tem o

\footnotetext{
${ }^{3}$ Neste trabalho, por razão conceitual far-se-á uso do termo Confirmação (Confirm) ao invés de Ack
} 
significado de que a Entidade requisitante sabe o que aconteceu com a primitiva (chegou sem erro, chegou com erro ou não chegou).

Serviços Piggybacking são serviços confiáveis, uma vez que a entidade requisitante recebe uma evidência da chegada dos dados ao destino, mas é interessante de ser utilizado em relações balanceadas nas quais as entidades participantes requisitam primitivas de dados nas mesmas quantidades. Nessas condições, este tipo de serviço apresenta a mesma funcionalidade dos Serviços Confirmados, mas ocupando bem menos recursos de comunicação (vazão, banda de comunicação, memória e processamento) e menor probabilidade de erro.

Considerando que a interface Socket é uma interface abstrata amplamente conhecida e que seus serviços são largamente utilizados nos dias atuais, então, visando interoperabilidade e portabilidade com a Internet, a arquitetura ETArch adotou esta interface para sua camada Communication, isto é, foi definida uma nova Família de Endereços (AF - Addess Family), denominada AF_MEHAR.

\subsection{Identificação e Endereçamento}

Identificação e Endereçamento são dois conceitos centrais para as comunicações entre entidades pares em um ambiente distribuído. Basicamente, as primitivas de dados são enviadas e recebidas por entidades, sendo que seus títulos (identificações) indicam "quem" são esses pares e os respectivos endereços indicam "onde" estão e como podem ser alcançadas (as entidades pares).

O título é responsável por designar a existência de uma entidade e permitir sua identificação de forma única, não ambígua e independente de topologia. 
O endereço, por sua vez, indica "onde" essa entidade está localizada e de posse dessa localização (SALTZER, 1993) permite construir um caminho para a comunicação entre essas entidades pares.

Na ETArch existe uma separação entre títulos e endereços. Títulos identificam precisamente uma entidade de forma independente de sua localização. Os endereços por sua vez são dependentes da localização. Se uma entidade se move, seu título não se altera, mas o endereço de seu PoA é alterado.

Conforme definido na seção 3.1.1, entidades trocam interações via workspace no qual cada uma é identificada unicamente por seus respectivos títulos. Considerando que existem entidades em todas as camadas da arquitetura e que as entidades de aplicação fazem uso dos serviços residentes em camadas subjacentes, então pode ser que o título de uma entidade de aplicação se derive de títulos associados a entidades subjacentes.

Por exemplo, do ponto de vista da ETArch, um host ou smartphone são entidades e, portanto, possuem títulos, então, programas ou sensores residentes nesses dispositivos podem ter um título único que é gerado a partir do título do dispositivo no qual está hospedado.

Observe-se que essa abordagem não fere o princípio da independência de localização do título, pois essas camadas podem ensejar aspectos da implementação e da arquitetura da máquina, mas não fixam a localização.

Da mesma forma, títulos de NEs e DTSAs podem ser gerados a partir, por exemplo, de um domínio, onde residem. Assim, é possível garantir a unicidade dos títulos que será única neste domínio. Essa estratégia pode ser utilizada em todas as camadas a fim de identificar as entidades e obter o seu titulo de forma única, facilitando o processo de nomeação para as entidades. A seguir apresenta-se como a identificação é tratada nas camadas Application 
e Communication.

Essa estratégia permite utilizar espaço de nomes nos quais entidades tais como hosts, smartphones ou sensores possuem títulos associados que são únicos em seus Namespaces. Títulos podem ser gerados a partir de Namespaces e acrescidos do ponto de acesso a serviço (SAP - Service Access Point) na interface da camada Communication (C-SAP).

Por exemplo, a Figura 21 a seguir representa o caso de um host cujo título é host :: myhost.mydomain no qual uma instância de uma aplicação de vídeo é instanciada e se registra no DTS com o título host :: myhost.mydomain $+C-$ $S A P$-identification. Considerando que cada workspace define um namespace que poderia ser identificável através do símbolo wks, então o título de uma instância de workspace poderia ser wks :: workspace - identification.

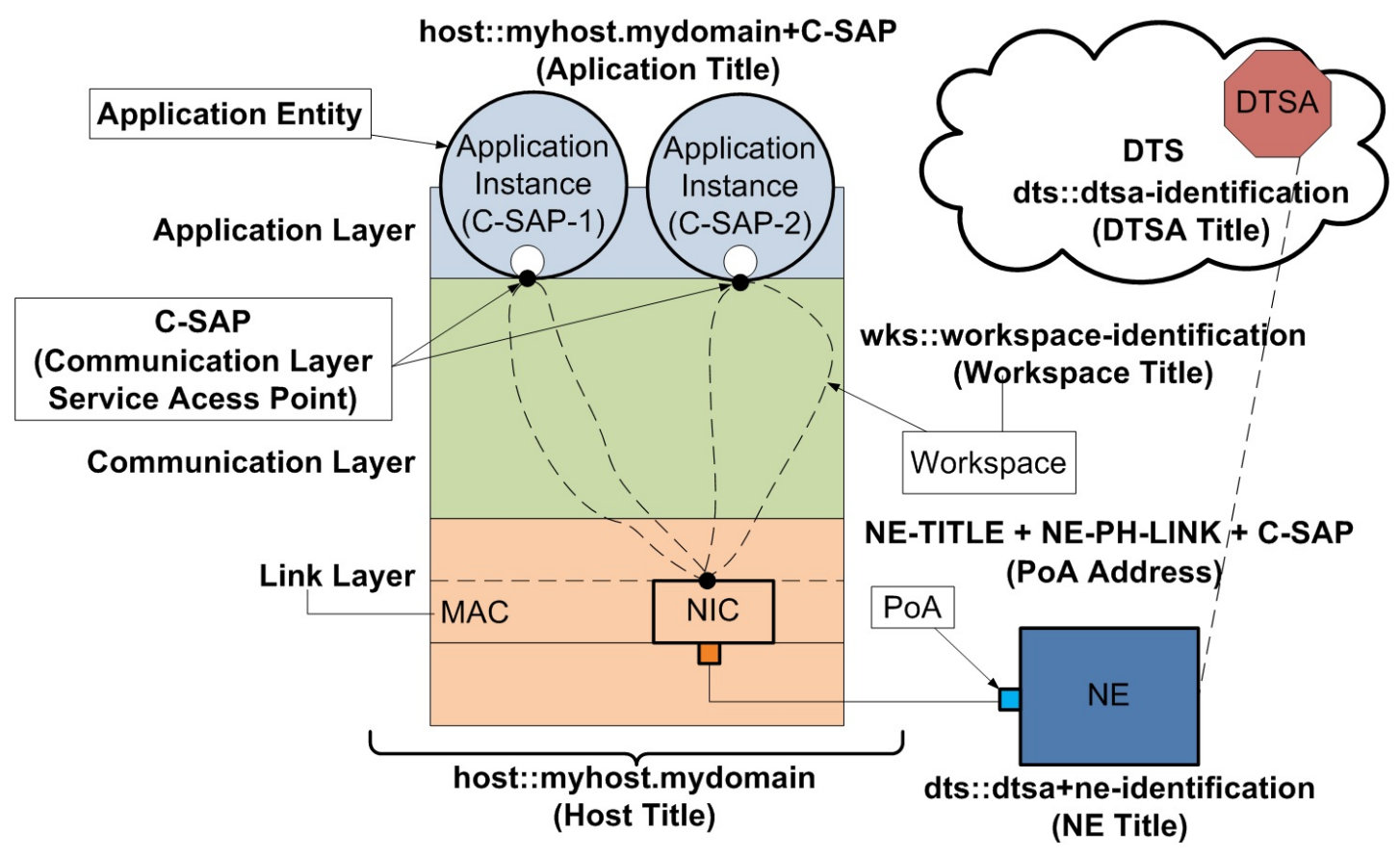

Figura 21: Identificação e Endereçamento.

Na Camada Communication, o título de workspace é representado por uma cadeia de bits originada a partir de sua identificação, por exemplo, através de um Hash do título. 
Cada DTSA possui seu título e neste caso será utilizado o namespace $d t s$, sendo possível identificar precisamente cada DTSA utilizando o título dts :: $d t s a-i d e n t i f i c a t i o n$. Da mesma forma cada NE possui seu título associado ao DTSA que o controla. Portanto, o título de um NE possui o seguinte formato $d t s:: d t s a+n e-i d e n t i f i c a t i o n$. Além de título, toda entidade tem um PoA, que endereça efetivamente uma entidade, com o seguinte formato: NE - TITLE+ $N E-P H-L I N K+C-S A P$, onde $N E-T I T L E$ é o título do NE e C - SAP seu ponto de acesso ao serviço. O valor $N E-P H-L I N K$ representa uma porta do NE.

\subsection{Protocolos ETArch}

Esta seção tem o objetivo de apresentar uma visão geral dos protocolos e suas situações em relação à Arquitetura ETArch, sendo que suas especificações detalhadas são objeto do Apêndice C.

A camada Communication tem dois conjuntos de serviços cada um dos quais associados aos planos de Controle e de Dados. Considerando que serviços são um dos elementos de protocolos, então são definidos protocolos para cada um dos planos.

Os protocolos do plano de Controle são responsáveis pelo ciclo de vida de entidades e workspaces e oferecem serviços tais como: registro de uma entidade no DTS; criação de um workspace; entrada e saída de entidades em workspace, etc. O Entity Title Control Protocol (ETCP) oferece serviços que envolvem interações entre entidades e DTSAs, enquanto o DTS Control Protocol (DTSCP) é responsável por interações entre DTSAs ou MDTSAs.

Para entidades do tipo NE, o DTS utiliza os serviços do protocolo OpenFlow. Na Figura 22 a seguir esses protocolos são identificados respectiva- 
mente por $P 1, P 2$ e $P 3$.

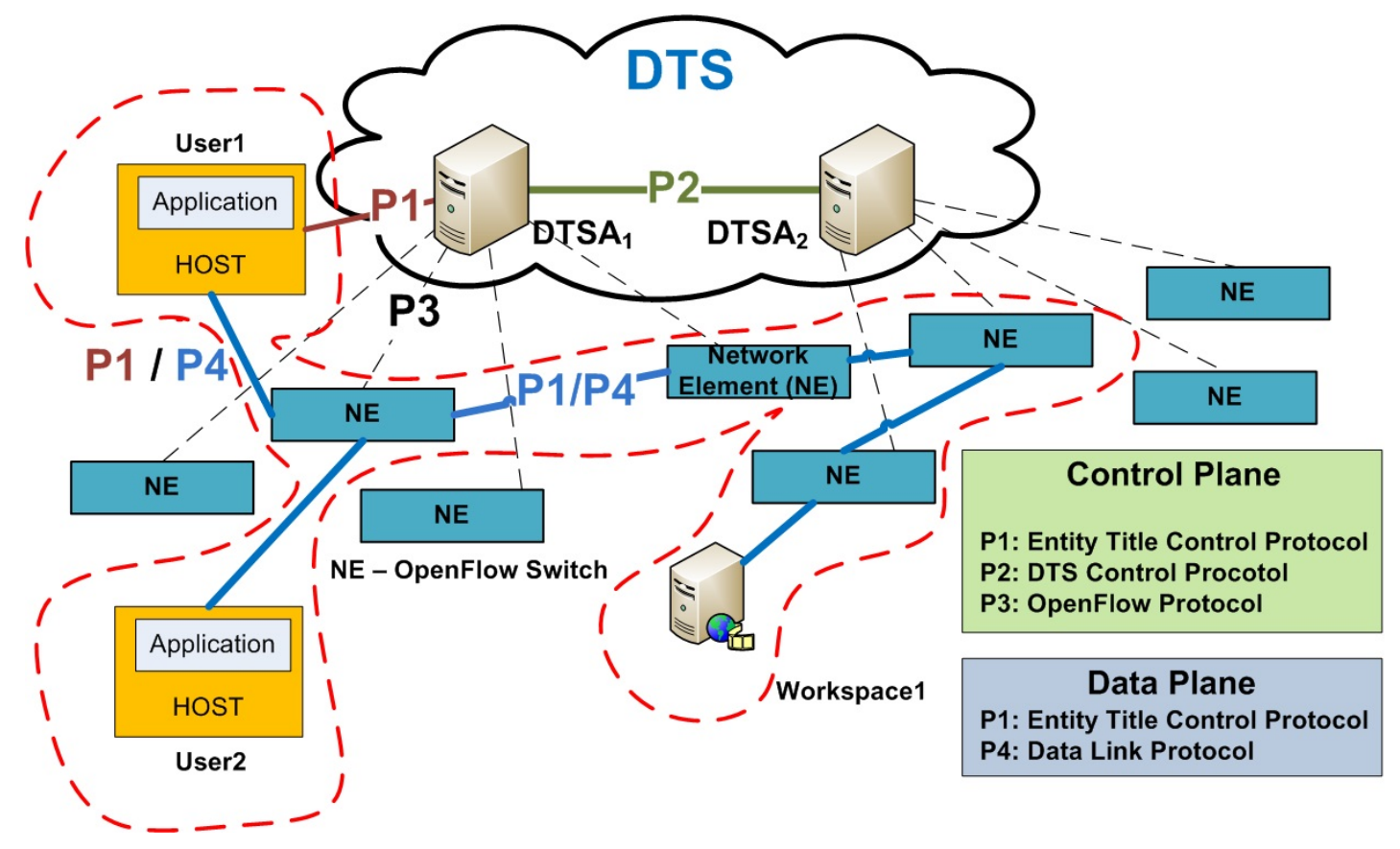

Figura 22: ETArch Protocols.

Considerando que uma entidade já foi registrada e anexada a um Workspace, então a entidade entra na fase de operação do plano de Dados (Data Plane), durante o qual as entidades de aplicação estão aptas a enviar/receber primitivas de dados para/do workspace. Durante a fase de operação do plano de Dados, a entidade de aplicação fará uso dos serviços do protocolo identificado como Data Link Protocol (P4).

\subsubsection{Entity Title Control Protocol}

O Entity Title Control Protocol (ETCP) é utilizado na camada Communication para disciplinar a relação entre uma entidade e o DTSA. A Tabela 4 a seguir sumariza os nomes e funcionalidades das primitivas deste protocolo.

Para que uma aplicação possa utilizar este protocolo, oferece-se uma API (Application Programming Interface) descrita na Tabela 5, que disponibiliza os serviços do ETCP a entidades da camada Application. 
Tabela 4: Primitivas do Entity Title Control Protocol(ETCP).

\begin{tabular}{|c|l|}
\hline PRIMITIVA & \multicolumn{1}{c|}{ FUNÇÃO } \\
\hline ENTITY-REGISTER & $\begin{array}{l}\text { Registra uma entidade no DTS. A fim de ser registrada uma } \\
\text { entidade deve apresentar o seu título, suas capacidades e } \\
\text { requisitos de comunicação. Antes de iniciar qualquer } \\
\text { comunicação a entidade deve realizar o seu registro. o } \\
\text { Registro é realizado pelo DTSA que controla o NE onde a } \\
\text { entidade está inicialmente conectada. }\end{array}$ \\
\hline ENTITY-UNREGISTER & $\begin{array}{l}\text { Remove uma entidade registrada do DTS e que não participa } \\
\text { de nenhuma comunicação }\end{array}$ \\
\hline WORKSPACE-ATTACH & $\begin{array}{l}\text { Anexa uma entidade ao workspace. Para realizar este } \\
\text { processo o DTSA realiza a configuração de todos os network } \\
\text { elements (NE) a fim de extender o workspace até o NE } \\
\text { através do qual a entidade está diretamente conectada. }\end{array}$ \\
\hline WORKSPACE-CREATE & $\begin{array}{l}\text { Cria um worskpace. Inicialmente está presente apenas no } \\
\text { uTSA local. Ao receber essa primitiva o DTSA pode executar } \\
\text { a Workspace Database de um determinado nível da rede. }\end{array}$ \\
\hline WORKSPACE-DELETE & $\begin{array}{l}\text { Remove um workspace, realizando a liberação de recursos } \\
\text { necessários e atualização de todos os NE envolvidos. }\end{array}$ \\
\hline WORKSPACE-DETACH & Remove uma entidade de um workspace. \\
\hline WORKSPACE-MODIFY & \begin{tabular}{l} 
Modifica as capacidades de um determinado workspace. \\
\hline
\end{tabular} \\
\hline
\end{tabular}

Tabela 5: ETCP Application Programming Interface.

\begin{tabular}{|c|l|}
\hline FUNÇÃO & \multicolumn{1}{|c|}{ DESCRIÇÃO DA CHAMADA } \\
\hline register & Responsável pelo registro de uma entidade \\
\hline unregister & $\begin{array}{l}\text { Responsável pela remoção do registro de uma entidade junto } \\
\text { ao DTS }\end{array}$ \\
\hline createWorkspace & $\begin{array}{l}\text { Utilizada por aplicação que é responsável pela criação de um } \\
\text { Workspace. }\end{array}$ \\
\hline attachWorkspace & $\begin{array}{l}\text { Utilizada por uma aplicação para se anexar a um Workspace já } \\
\text { existente. }\end{array}$ \\
\hline detachWorkspace & $\begin{array}{l}\text { Utilizada por uma aplicação a fim de se separar de um } \\
\text { Workspace. }\end{array}$ \\
\hline deleteWorkspace & $\begin{array}{l}\text { Utilizada pela aplicação que criou o workspace a fim de } \\
\text { removê-lo do DTS. }\end{array}$ \\
\hline send & Utilizada para o envio de dados para um Workspace. \\
\hline receive & Utilizada para o recebimento de dados de um Workspace. \\
\hline
\end{tabular}

\subsubsection{DTS Control Protocol}

O DTS Control Protocol (DTSCP) é responsável pelas relações de controle internas ao DTS envolvendo DTSA-DTSA, DTSA-MDTSA e MDTSA-MDTSA. 
A Tabela 6 apresenta as primitivas definidas para este protocolo. Assim como no caso do protocolo ETCP, oferece-se uma API, cujos serviços seguem a mesma lógica de nomeação.

Tabela 6: Primitivas do DTS Control Protocol(DTSCP).

\begin{tabular}{|c|c|}
\hline PRIMITIVA & FUNÇÃO \\
\hline WORKSPACE-LOOKUP & $\begin{array}{l}\text { Utilizada pelo DTSA junto ao seus Resolvers. Ocorre na } \\
\text { presença de uma primitiva WORKSPACE-ATTACH, caso o } \\
\text { DTSA onde a entidade está registrada não possua } \\
\text { informações sobre este workspace. Essa operação inicia-se } \\
\text { no nível onde o DTSA está localizado e pode ser } \\
\text { encaminhada até o nível raiz. }\end{array}$ \\
\hline DTSA-REGISTER & $\begin{array}{l}\text { Responsável por registar um DTSA. Ao registrar-se o DTSA } \\
\text { deve incluir no DTSA Resolver Database uma entrada } \\
\text { indicando quais são os seus resolvers. O registro é feito no } \\
\text { Master DTSA do mesmo nível do DTSA que se registra }\end{array}$ \\
\hline WORKSPACE-ADVERTISE & $\begin{array}{l}\text { Insere, remove ou atualiza o Workspace Database, } \\
\text { indicando em qual DTSA um dado workspace está } \\
\text { presente. Este serviço recebe deve receber o nível que } \\
\text { indica qual será a visibilidade do workspace. Essa operação } \\
\text { inicia-se no Master DTSA do mesmo nível onde o DTSA } \\
\text { está localizado e pode ser encaminhada até o nível raiz. }\end{array}$ \\
\hline DTS-MESSAGE & $\begin{array}{l}\text { Permite a comunicação entre diferentes DTSAs no âmbito } \\
\text { do DTS. Caso o DTSA de origem conheça previamente o } \\
\text { caminho até o DTSA de destino, este caminho está } \\
\text { presente no cabeçalho da mensagem. Caso contrário, a } \\
\text { mensagem será encaminhada para os seus Resolvers, até } \\
\text { que um deles saiba calcular o caminho até o DTSA de } \\
\text { destino. Caso um MTDSA do nível raiz não possa calcular o } \\
\text { caminho até o destino então o envio desta mensagem } \\
\text { falhará. }\end{array}$ \\
\hline
\end{tabular}

Esta seção apresentou as camadas e entidades da arquitetura ETArch e os detalhes sobre os elementos dos protocolos, respectivos serviços, comportamentos e parametrização serão apresentados no Apêndice C.

\subsection{Aspectos da Rede}

Esta seção tem o objetivo de introduzir aspectos relativos à rede, principalmente aqueles aspectos nos quais a Arquitetura ETArch se difere dos tratamentos tradicionais das arquiteturas de redes de computadores como é o 
caso da Arquitetura Internet.

Desse modo, dois aspectos são particularmente importantes, pois são fundamentais para as redes e na ETArch introduzem uma nova abordagem: Roteamento e Mobilidade. Por razões óbvias, as comparações são feitas com a Arquitetura Internet.

\subsubsection{Roteamento}

$\mathrm{Na}$ Arquitetura Internet a funcionalidade de roteamento é dividida entre os planos de Controle e de Dados. O plano de Controle da Arquitetura Internet é desempenhado por protocolos IGP (Interior Gatewat Protocol) - tais como OSPF (Open Shortest Path First) e RIP (Routing Information Protocol) - e EGP (Exterior Gateway Protocol) - tal como o BGP (Border Gateway Protocol). O plano de Dados é desempenhado pelo protocolo IP (Internetworking Protocol).

Os protocolos do plano de Controle abastecem as tabelas de rotas residentes na camada de Rede da Arquitetura Internet - que é a primeira parte do roteamento - enquanto o protocolo IP (plano de Dados) executa a segunda parte do roteamento ao escrutinar a tabela de rotas até achar um caminho na direção do destino e, então, enviar o pacote.

Observe que ao executar roteamento in-band, a segunda parte do roteamento, o protocolo IP sofre uma perda considerável de tempo no escrutínio da tabela de rota, além de introduzir a possibilidade de roteamento circular no qual um pacote estabelece um círculo por um conjunto de roteamentos até que o tempo de vida (TTL - Time To Live) do pacote se expire e o pacote seja descartado. O roteamento in-band faz com que o pacote passe por este procedimento em cada hop da rede. 
Fazendo uma analogia, seria equivalente a uma pessoa que pergunta em cada esquina qual a direção para chegar a um determinado destino.

A Arquitetura ETArch adota dois diferenciais em relação ao descrito anteriormente: 1) a filosofia de roteamento out-of-band no qual a descoberta do caminho é feito a priori e é mantido durante todo o ciclo de vida do workspace; 2) considerando que o destino é o workspace, então o roteamento deve descobrir o local mais próximo à entidade que quer ser ligada ao workspace.

Desse modo, há dois benefícios na filosofia adotada pela Arquitetura ETArch, sendo: 1) o tempo de switching é muito inferior ao tempo de roteamento; e, 2) o workspace em geral pode estar mais 'próximo' da entidade requisitante do que da entidade que originalmente criou o workspace.

\subsubsection{Mobilidade}

Dois fatores limitam a mobilidade em aplicações baseadas na Arquitetura Internet. O primeiro fator, e mais importante, está relacionado ao endereçamento IP que é hierárquico e tem de ser alterado a cada vez que o nó muda de rede. O segundo fator advém da filosofia de comunicação da Internet na qual o caminho virtual (Virtual Path) entre os end-points é definido na resolução de nomes nos instantes iniciais da comunicação.

Sem entrar nos méritos de desempenho e dos diversos tipos de tráfegos, esses fatores são menos sensíveis, e podem ser razoavelmente mitigados ${ }^{4}$, para aplicações desenvolvidas no modelo stateless, no qual as transmissões não guardam estado (sem memória), como é o caso da maioria dos acessos a páginas Web, por exemplo.

Contudo, aplicações desenvolvidas no modelo stateful, como é o caso de

\footnotetext{
${ }^{4}$ Por exemplo, DHCP diminui razoavelmente o impacto da troca de endereço
} 
aplicações orientadas a conexão, por exemplo uma transmissão de video ${ }^{5}$, não funcionam apropriadamente se houver troca de endereço de end-points de comunicação.

O conceito de Workspace se propõe a resolver a questão da mobilidade, pois as entidades de aplicação enviam ou recebem suas comunicações para/de ele. Considerando que o título das entidades independe de localização, o endereço dos end-points (dispositivos móveis) se torna irrelevante. É responsabilidade do DTS manter o Workspace atualizado para atender aos requisitos das entidades pares.

Outro aspecto a se ressaltar é que o Workspace passa a ser um barramento lógico que pode ser estabelecido de maneiras variadas, em relação aos meios físicos, sendo que as entidades de aplicação podem residir em equipamentos conectados em redes cabeadas (meios metálico ou óptico) ou residir em equipamentos sem fio.

Outro fator a ser frisado é que há diversas tecnologias de redes sem fios tanto para redes de dados (WiFi, WiMax, etc) quanto para redes de telecomunicações (3G, 4G, LTE, etc). Os dispositivos móveis, notadamente smartphones, possuem em geral pelos menos duas interfaces para tecnologias sem fio. No caso da Arquitetura Internet, isto significa que ao trocar de rede sem fio, muitas vezes, o dispositivo não troca apenas de endereço IP, troca também de interface de comunicação em função da tecnologia wireless.

A Arquitetura ETArch utiliza o conceito de Vertical Handover, que permite a mobilidade entre tecnologias de enlaces wireless diferentes, com a consequente troca de interface no dispositivo móvel, padronizado pela norma IEEE 802.21 (MIH - Media Independent Handover) que é uma materialização de SDN para redes sem fio.

${ }^{5}$ O RTCP ou H.323 (plano de controle) são orientados a conexão 
Portanto, quando se menciona anteriormente que o Workspace pode ser estabelecido de maneiras variadas em relação aos meios físicos, no que tange as tecnologias sem fio, a mobilidade pode ser horizontal (mesma tecnologia sem fio) ou vertical (tecnologias sem fio diferentes). 


\section{ARQUITETURA ETARCH: IMPLEMENTAÇÃO E ANÁLISE}

Este capítulo apresenta reflexões sobre a implementação da Arquitetura ETArch e analisa os resultados obtidos nesta jornada. Particularmente sobre os resultados, oportuno é ressaltar que a análise comparativa foi parcialmente alcançada em função de não haver arquitetura clean slate aberta e disponível para experimentação.

Outrossim, pelas razões apresentadas em 2.1 (Aspectos de Endereçamento em Redes de Computadores) e 3.4 (Idenfificação e Endereçamento), considerando que as características de endereçamento IP Multicast não são praticamente utilizadas por aplicações multi-peer pelas razões apresentadas anteriormente, as análises aqui realizadas comparam as facilidades do conceito de Workspace com aplicações da Arquitetura Internet baseadas em comunicações ALM (Application Layer Multicast) e Unicast.

Análises sobre os resultados de comunicações Unicast, orientadas ou não orientadas a conexão, comparadas a comunicações via Workspace são pertinentes, pois este é um super conjunto daquelas e, então, há que se mensurar suas eficácias. Em 2.1.1 (Aspectos Conceituais e Filosóficos de Endereçamento) foram introduzidas as bases para tal comparação.

A estratégia de implementação da Arquitetura ETArch baseou-se na separação dos Planos de Dados e de Controle, cuja essência filosófica já era 
debatida no seio do Programa MEHAR (MEHAR, 2011) desde 2006. Este capítulo descreve, portanto, a implementação baseada em SDN da arquitetura ETArch e apresenta os resultados obtidos de forma experimental. Finalmente é apresentada discussão acerca de alguns resultados decorrentes deste trabalho.

\subsection{Aspectos de Implementação}

Através de uma abordagem baseada em prototipação rápida (TRIPP; BICHELMEYER, 1990) foi possível implementar os conceitos apresentados na seção 3.1. O método de pesquisa nesta etapa constituiu portanto em: definir o objetivo; construir um protótipo baseado em software; utilizar o protótipo através da execução de diferentes experimentos; observar os resultados e inferir conclusões; realizar as modificações necessárias; e iniciar um novo ciclo de prototipagem. Esta seção descreve dois diferentes estágios de implementação da arquitetura ETArch.

O componente central na arquitetura é o DTSA (definido na Seção 3.1.4) e, portanto, sua implementação é crucial para testar a arquitetura ETArch. O objetivo era testar a viabilidade de uso de Título na identificação, no endereçamento e na comunicação através de Workspace. A tarefa do DTSA é coordenar os NEs e alterar o seu comportamento a fim de permitir a manuteção do Workspace. Por outro lado, em um switch OpenFlow, o controlador OpenFlow é o responsável pelo estado da Flow Table (conforme apresentado na Seção 2.2.3) sendo capaz de alterar ao longo do tempo o encaminhamento de primitivas em cada NE. Desta forma, um DTSA pode ser implementado a partir de um controlador OpenFlow. Para a implementação realizada neste trabalho, foi utilizado o controlador FloodLight (BIG SWITCH, 2012b). 
FloodLight é uma implementação de código aberto de um controlador OpenFlow que combina facilidades de desenvolvimento com a capacidade de ser implantado em ambientes reais, sobre redes em produção. Através da definição de novos módulos (BIG SWITCH, 2012a) é possível estender seu comportamento. Cada módulo "escuta" a comunicação com o switch OpenFlow e provê um comportamento específico.

A extensão do FloodLight consiste em um novo módulo que instancia o DTSA a fim de tratar as primitivas do protocolo ETCP (apresentado na Seção 3.5.1 e detalhado no Apêndice C.2).

No switch Openflow, para qualquer primitiva recebida, sempre que não houver uma regra na flow table do switch, ela será encaminhada para o controlador. Neste caso, sempre que uma primitiva ETCP for recebida pelo switch, ela será encaminhada ao controlador, i.e., ao DTSA, dentro de uma mensagem OFPT_PACKET_IN. Ao receber essa mensagem, o módulo do DTSA verifica se se trata de uma das primitivas definidas para o ETCP. Se for, o módulo realiza o tratamento da primitiva e devolve o resultado através de uma mensagem OPFT_PACKET_OUT, que, então, é encaminhada para a entidade que inicialmente enviou a primitiva ao switch.

A fim de dar vida ao conceito do Workspace, o DTSA modifica o conteúdo da Flow Table dos switches envolvidos, utilizando mensagens OPFT_FLOW_MOD. A Figura 23 apresenta este processo, enquanto a Tabela 7 apresenta as primitivas do ETCP (inicialmente apresentadas na Tabela 4) e sua relação com o OpenFlow. Deve ser ressaltado que a título de simplificação, as primitivas OPFT_PACKET_OUT foram omitidas da Figura 23.

A partir desta implementação, dentro do processo de prototipagem rápida utilizado, novas versões do DTSA foram criadas a cada iteração. 


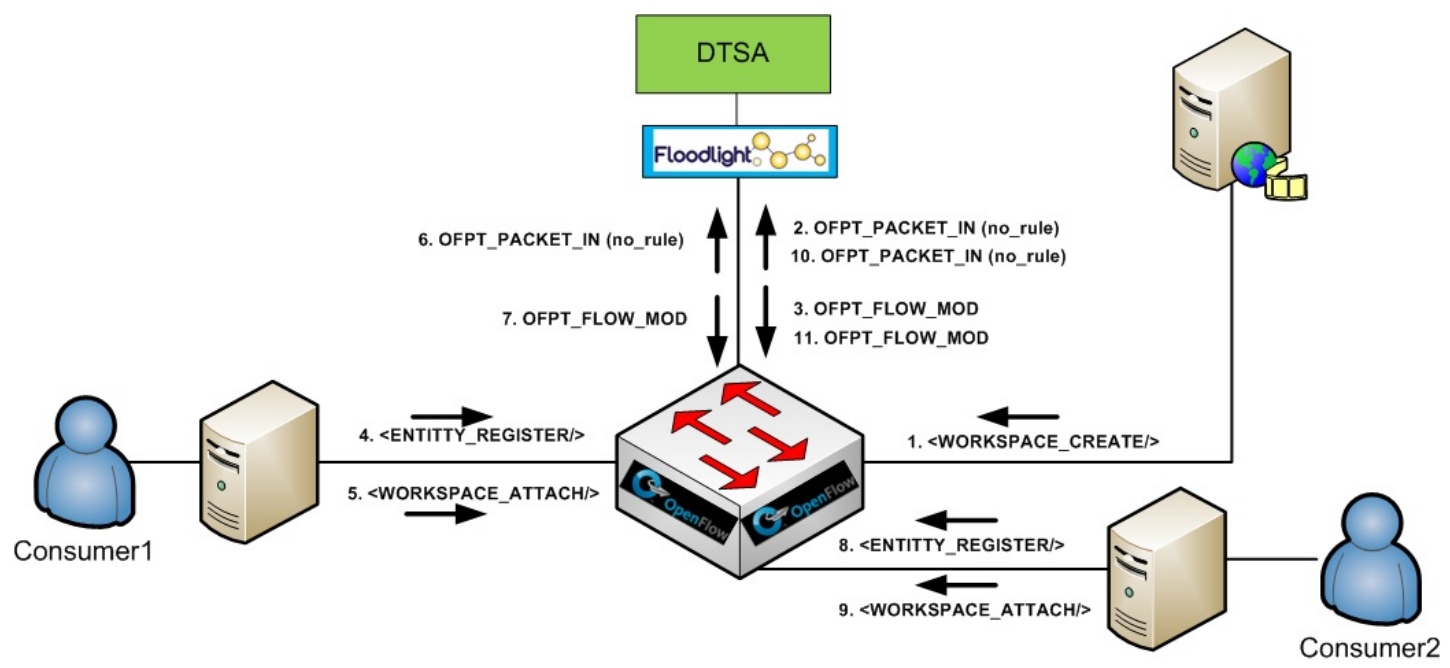

Figura 23: Primitivas ECTP e OpenFlow Trocadas a Fim de Criar e Adaptar o Workspace.

Tabela 7: Primitivas do ETCP e sua Relação com o OpenFlow.

\begin{tabular}{|c|c|c|}
\hline PRIMITIVA & FUNÇÃO & RELAÇÃO COM O OPENFLOW \\
\hline ENTITY-REGISTER & $\begin{array}{l}\text { Registra uma entidade no DTS. A fim de ser registrada uma } \\
\text { entidade deve apresentar o seu título, suas capacidades e } \\
\text { requisitos de comunicação. Antes de iniciar qualquer } \\
\text { comunicação a entidade deve realizar o seu registro. o } \\
\text { Registro é realizado pelo DTSA que controla o NE onde a } \\
\text { entidade está inicialmente conectada. }\end{array}$ & $\begin{array}{l}\text { A primitiva é encapsulada em um OFPT_PACKET_IN que então } \\
\text { é enviado ao DTSA, utilizando o protocolo OpenFlow. A } \\
\text { reposta é devolvida ao Switch em um } \\
\text { OFPT_PACKET_OUT }\end{array}$ \\
\hline ENTITY-UNREGISTER & $\begin{array}{l}\text { Remove uma entidade registrada do DTS e que não participa } \\
\text { de nenhuma comunicação }\end{array}$ & O mesmo comportamento apresentado pelo ENTITY-REGISTER \\
\hline WORKSPACE-ATTACH & $\begin{array}{l}\text { Anexa uma entidade ao workspace. Para realizar este processo } \\
\text { o DTSA realizará a configuração de todos os network elements } \\
\text { (NE) a fim de extender o workspace até o NE através do qual a } \\
\text { entidade está diretamente conectada. }\end{array}$ & $\begin{array}{l}\text { A primitiva é encapsulada em um OFPT_PACKET_IN que então } \\
\text { é enviado ao DTSA, utilizando o protocolo OpenFlow. A } \\
\text { resposta é devolvida ao Switch em um OFPT_PACKET_OUT. } \\
\text { Utilizando mensagens OFPT_FLOW_MOD, a porta física em } \\
\text { que a entidade está conectada naquele momento é } \\
\text { adicionada à Flow Table, como um destino para os dados que } \\
\text { serão transmitidos por um Worskpace }\end{array}$ \\
\hline WORKSPACE-CREATE & $\begin{array}{l}\text { Cria um worskpace. Inicialmente está presente apenas no } \\
\text { DTSA local. Ao receber esta primitiva o DTSA poderá executar } \\
\text { uma divulgação (advertise ), enviando suas informações para a } \\
\text { Workspace Database de um determinado nível da rede. }\end{array}$ & $\begin{array}{l}\text { A primitiva é encapsulada em um OFPT_PACKET_IN que então } \\
\text { é enviado ao DTSA, utilizando o protocolo OpenFlow. A } \\
\text { resposta é devolvida ao Switch em um OFPT_PACKET_OUT. }\end{array}$ \\
\hline WORKSPACE-DELETE & $\begin{array}{l}\text { Remove um workspace, realizando a liberação de recursos } \\
\text { necessários e atualização de todos os NE envolvidos. }\end{array}$ & $\begin{array}{l}\text { A primitiva é encapsulada em um OFPT_PACKET_IN que então } \\
\text { é enviado ao DTSA, utilizando o protocolo OpenFlow. A } \\
\text { resposta é devolvida ao Switch em um OFPT_PACKET_OUT. } \\
\text { Utilizando mensagens OFPT_FLOW_MOD, a regra na Flow } \\
\text { Table associada ao Workspace é removida. Esta remoção afeta } \\
\text { todos os switches que estão inseridos neste Workspace }\end{array}$ \\
\hline WORKSPACE-DETACH & Remove uma entidade de um workspace. & $\begin{array}{l}\text { A primitiva é encapsulada em um OFPT_PACKET_IN que então } \\
\text { é enviado ao DTSA, utilizando o protocolo OpenFlow. A } \\
\text { resposta é devolvida ao Switch em um OFPT_PACKET_OUT. } \\
\text { Utilizando mensagens OFPT_FLOW_MOD, a porta física em } \\
\text { que a entidade está conectada naquele momento é removida } \\
\text { da Flow Table, como um destino para os dados que serão } \\
\text { transmitidos por um Worskpace }\end{array}$ \\
\hline WORKSPACE-MODIFY & Modifica as capacidades de um determinado workspace. & $\begin{array}{l}\text { A primitiva é encapsulada em um OFPT_PACKET_IN que então } \\
\text { é enviado ao DTSA, utilizando o protocolo OpenFlow. A } \\
\text { resposta é devolvida ao Switch em um OFPT_PACKET_OUT. }\end{array}$ \\
\hline
\end{tabular}

A seguir é apresentada a versão final do DTSA, que foi construída utilizando-se um modelo de componentes especializados para o desenvolvimento de 
aplicações baseadas em eventos, conhecido como JAIN SLEE (Java APIs for Integrated Networks - Service Logic Execution Environment).

O modelo de componentes do JAIN SLEE permite a criação de aplicações que possuam requisitos como alta vazão, baixa latência, escalabilidade e disponibilidade (FEMMINELLA et al., 2011). Atualmente várias operadoras de telecomunicações possuem serviços implementados via JAIN SLEE. A implementação aberta do JAIN SLEE é conhecida como Mobicents (MOBICENTS, 2012).

Dois componentes centrais na especificação do JAIN SLEE são os Service Building Blocks (SBB) e Resource Adaptor (RA). SBB contém a aplicação e a lógica do serviço. Cada SBB é composto de um ou mais SBBs filhos que são organizados em um grafo de componentes. Um SBB se registra para disparar e capturar certos tipos de eventos.

De acordo com a especificação do JAIN SLEE, um Recurso (Resource) representa um sistema que é externo ao JAIN SLEE. O Resource Adaptor (RA) é um componente capaz de adaptar este recurso para uso em conjunto com o JAIN SLEE, assim sendo, vários protocolos do plano de controle, comumente utilizados na pilha de protocolos das operadoras de telecomunicações, podem ser "plugados" no JAIN SLEE, facilitando o desenvolvimento de novos serviços.

Um RA recebe mensagens deste sistema externo e as encaminha como eventos que são produzidos pelo RA. O RA pode, além disso, consumir eventos criados pelos serviços que estão em execução no JAIN SLEE.

O controlador é um recurso do ponto de vista do JAIN SLEE. Foi desenvolvido um RA para o controlador OpenFlow, pois não havia um RA disponível à época. O OpenFlow Resource Adaptor (OpenFlowRA), apresentado na Fi- 
gura 24, é responsável pela interação com os componentes do DTSA que são executados dentro do JAIN SLEE. Os eventos são capturados pelo SBB de acordo com a configuração de cada serviço dos protocolos ETCP e DTSCP apresentados no Apêndice C.

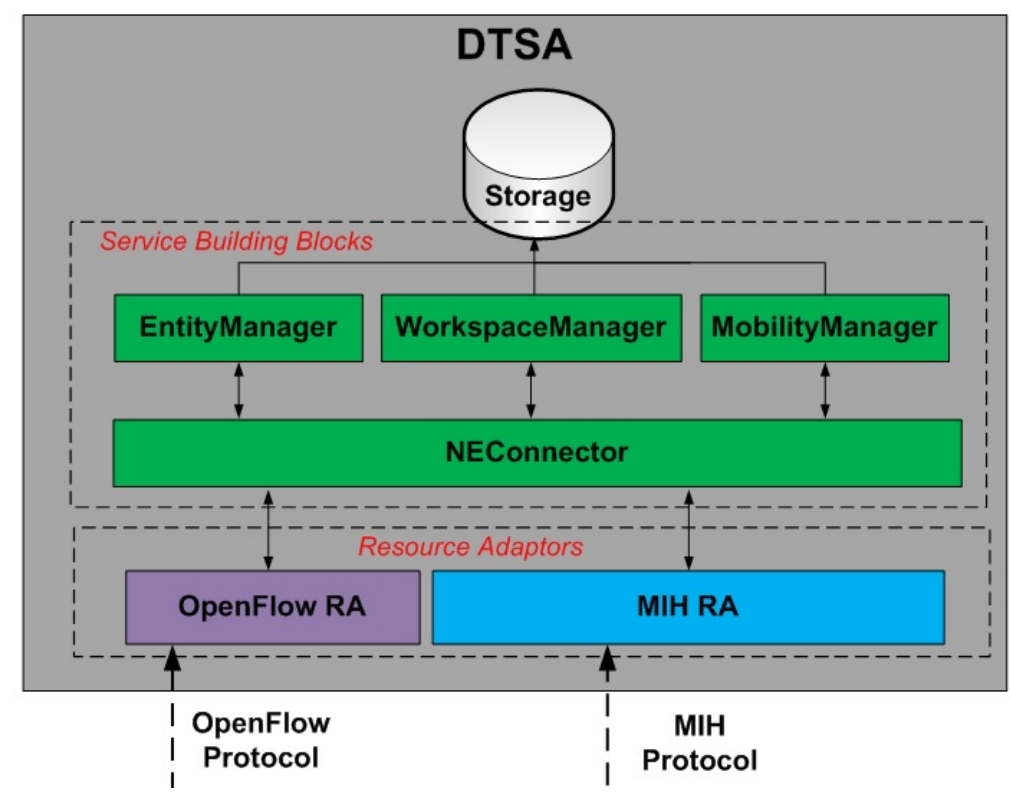

Figura 24: Componentes da Arquitetura do DTSA Implantados no JAIN SLEE.

Quando o OpenFlowRA é ativado pelo JAIN SLEE, ele também inicia o controlador FloodLight. Após este ponto, todas as mensagens do OpenFlow recebidas são convertidas em eventos que são enviados para o JAIN SLEE. Da mesma forma, os componentes do DTSA que são executados dentro do JAIN SLEE, podem disparar eventos para OpenFlowRA que, neste caso, correspondem às mensagens OFPT_FLOW_MOD e OFPT_PACKET_OUT que os captura e então os encaminha para os switches utilizando o protocolo OpenFlow.

Considerando que a ETArch também leva a abstração do controle às redes sem fio, foi desenvolvido um Resource Adaptor para o MIH (MIHRA) no âmbito deste projeto. O MIHRA utiliza a implementação de código aberto do IEEE 802.21: o ODTONE (AGUIAR et al., 2011). Desta forma abre-se o caminho 
para utilizar as capacidades do IEEE 802.21 a fim de otimizar a mobilidade de entidades ligadas a um determinado Workspace.

A Figura 24 apresenta os SBBs criados a fim de implementar o DTSA, sendo que cada um deles lida com os principais aspectos da arquitetura que são os controles de: entidades (EntityManager); Workspaces (WorkspaceManager); e mobilidade dentro do Workspace (MobilityManager). O SBB NEConnector é responsável por desacoplar estes elementos da arquitetura de comunicação com os NEs através dos protocolos OpenFlow ou MIH. O módulo Storage, por sua vez, é responsável pela persistência das informações sobre entidades e Workspaces e também pelos componentes DTS Resolver Database e Workspace Database, caso o DTSA assuma o papel de um MDTSA.

Estes SBBs são responsáveis pelos comportamentos apresentados no Apêndice $C$, seções C.2 e C.3, que detalha o comportamento das primitivas dos protocolos ETCP e DTSCP respectivamente.

\subsection{Avaliação Experimental}

Diferentes tipos de experimentos foram conduzidos durante o ciclo de desenvolvimento da ETArch com o objetivo de avaliar o conceito do Workspace. Esta seção descreve dois desses experimentos, os resultados associados e, além disso, apresenta uma avaliação da sinalização utilizada em um cenário de mobilidade no qual um Workspace se estende por diferentes DTSAs.

\subsubsection{Comunicação Baseada no Workspace}

O objetivo desta seção é realizar uma comparação da abordagem de comunicação da ETArch, com uma comunicação típica da arquitetura TCP/IP, como a utilizada, por exemplo, pelo YouTube. Neste caso foi utilizada a imple- 
mentação do DTSA baseada no FloodLight (apresentada na Seção 4.1).

Para realizar a experimentação foi utilizado o Mininet (LANTZ; HELLER; McKeown, 2010), que é um sistema de prototipagem rápida para redes baseadas em OpenFlow. Através do uso das capacidades de virtualização do Linux é possível simular diferentes hosts, switches e suas ligações. O Mininet possui uma Command Line Interface (CLI) que permite a interação com estes diferentes hosts e uma Application Programming Interface (API), baseada em Python, viabilizando a criação de diversas topologias.

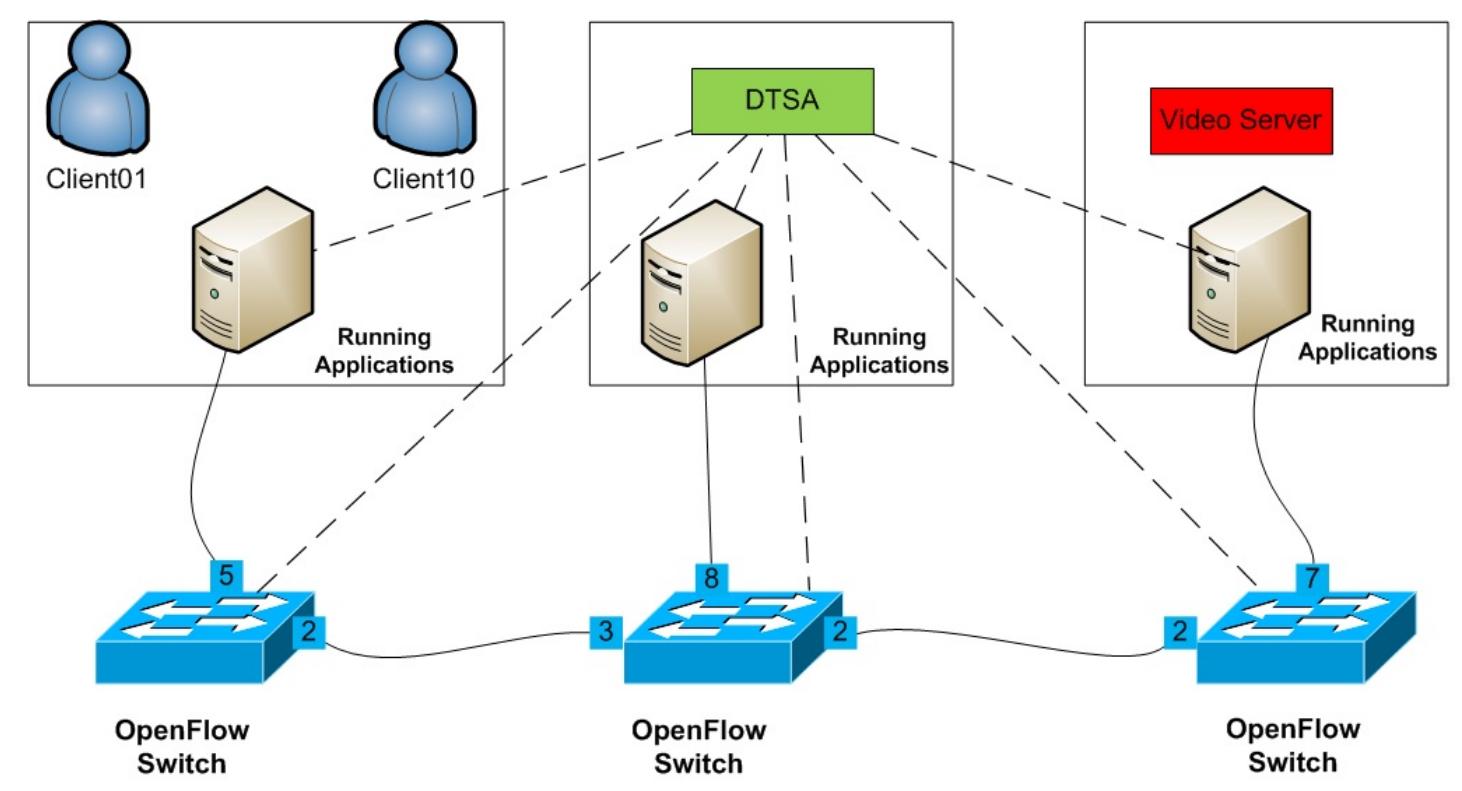

Figura 25: Cenário Utilizado para Experimentação.

Foi definida a topologia apresentada na Figura 25 como o primeiro cenário. No lado direito da figura, existe um servidor onde está implantada uma aplicação que distribui um vídeo através de uma stream baseada em MJPEG. Este servidor está conectado a um switch, que, por sua vez, está ligado a um outro switch intermediário, que, então, está conectado a um terceiro switch à esquerda na figura.

No switch à esquerda, um número variável de aplicações clientes deste servidor de vídeo foram instanciadas durante o experimento. Apesar de ser 
uma topologia simples, ela reflete uma situação comum onde um servidor e um ou mais clientes estão separados por um grupo de um ou mais switches.

A fim de comparar a comunicação baseada no Workspace com uma comunicação típica da arquitetura TCP/IP, duas diferentes versões do servidor foram criadas. Uma baseada nos protocolos UDP e IP e a outra baseada na abordagem proposta neste trabalho. Em essência as aplicações são as mesmas, sendo que a diferença entre elas é a forma com que os sockets são criados e utilizados.

Em ambos os casos, na camada de aplicação, uma mensagem baseada no protocolo Real-time Transport Protocol (RTP) (SCHULZRINNE et al., 2003) é criada. No primeiro caso, porém, um socket baseado em Datagrama é criado para o envio dessa mensagem. Por sua vez, na aplicação de vídeo que utiliza o Workspace, é criado um DTSSocket, que, neste caso, é baseado em Raw Sockets, que não utiliza a pilha TCP/IP e, diretamente, cria um frame que é enviado diretamente através do meio físico.

Na realidade, o frame criado por meio do DTSSocket utiliza o cabeçalho padrão da Ethernet, mas não da forma canônica, ou seja, ao invés disto, o campo Source Address contém os bits mais significativos do Título do Worksapce enquanto o campo Destination Address contém os bits menos significativos.

Foi iniciada a aplicação capaz de servir vídeo e, durante este experimento, um número variável de clientes foi instanciado a fim de interagir com este servidor, requisitando dados. Para a aplicação baseada na pilha TCP/IP, à medida que o número de clientes cresce, o consumo da banda de comunicação cresce da mesma forma, visto que para cada cliente, é instanciada uma stream de video. 


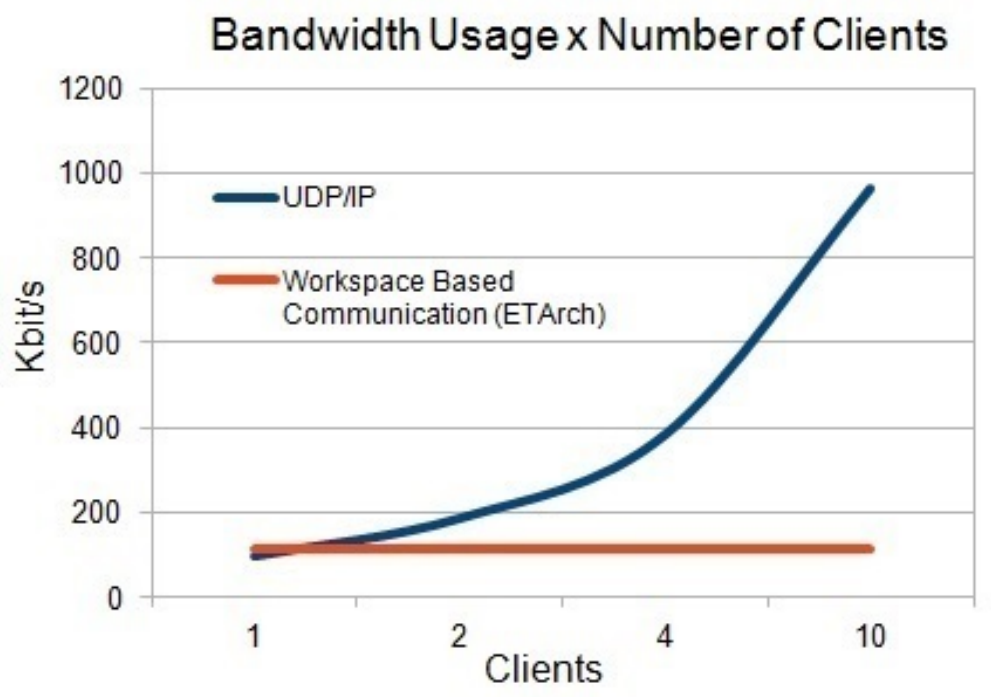

(a) Uso da Banda de Dados na Origem

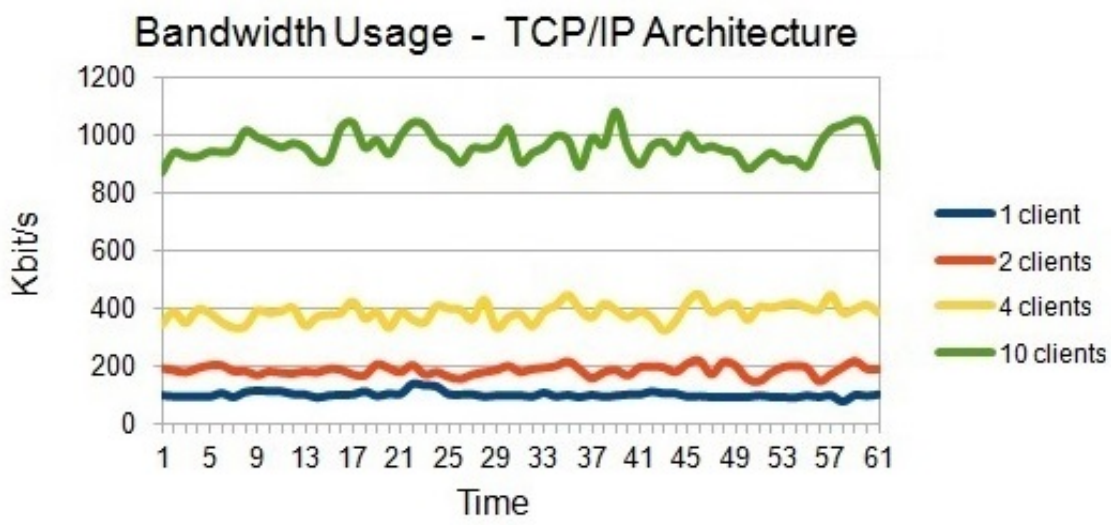

(b) Arquitetura TCP/IP

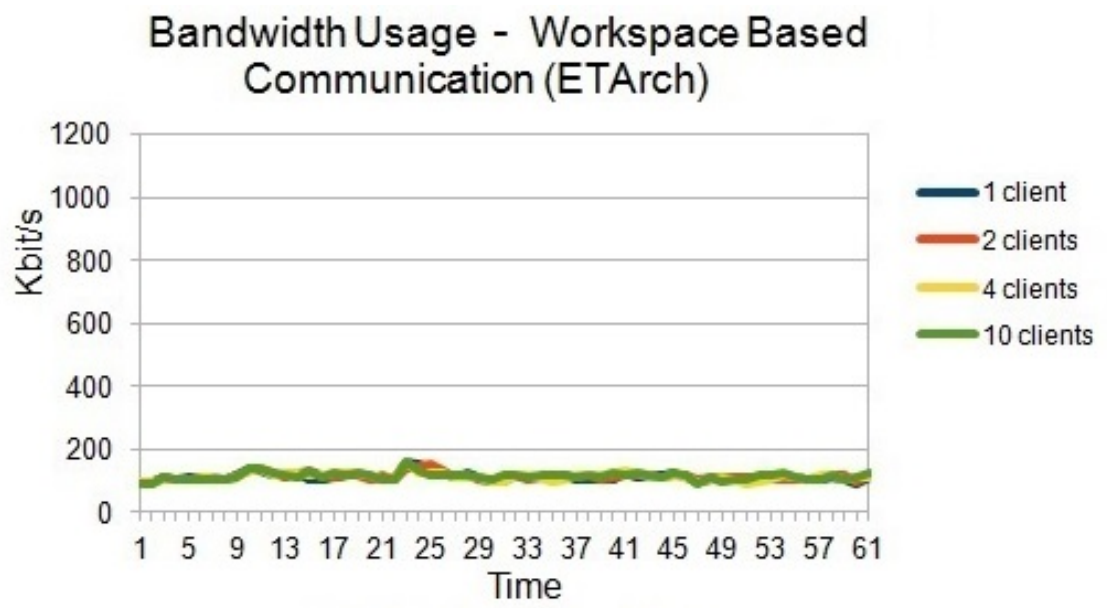

(c) Arquitetura ETArch - Comunicação Baseada no Workspace

Figura 26: Comparação no uso da Banda de Dados entre Arquiteturas TCP/IP e ETArch. 
Por outro lado, na aplicação baseada no Workspace, o uso da banda de comunicação na origem permanece constante, independentemente do número de clientes instanciados. Isto acontece pelo fato de que os dados são enviados para o Workspace e o cliente conecta-se ao Workspace e não diretamente ao servidor. A Figura 26(a) mostra o uso da banda de dados utilizada durante a comparação.

As Figuras 26(b) e 26(c), mostram o uso da banda de comunicação em cada situação para um número fixo de clientes. A aplicação baseada em UDP/IP apresenta um consumo superior à medida que o número de clientes aumenta, enquanto na comunicação baseada no workspace o consumo permanece constante, independente do número de clientes.

\subsubsection{Mobilidade Através do Workspace}

Esta seção descreve um caso e cenário experimental onde o foco é a mobilidade das entidades que estão anexadas a um Workspace. O mesmo descreve a sinalização de controle envolvida em um caso particular de mobilidade entre diferentes DTSAs. O caso apresentado mostra como a abstração do controle de redes de acesso com fio, através do protocolo OpenFlow e das redes de acesso sem fio, através do protocolo $\mathrm{MIH}$, previstas na arquitetura ETArch, o conceito do workspace e a identificação e endereçamento por título podem favorecer diferentes cenários de mobilidade.

A Figura 27 apresenta a sequência de primitivas do protocolo ETCP, utilizado pelas entidades para criar ou anexar-se a um Workspace a fim de comunicar-se. Uma entidade, neste caso o VIDEO SERVER, deseja prover uma nova mídia e para isto utiliza a primitiva WORKSPACE_CREATE.req. Esta primitiva é encaminhada pelo NE até o DTSA, utilizando uma mensagem OFPT_PACKET_IN do protocolo OpenFlow. O DTSA ao receber esta 


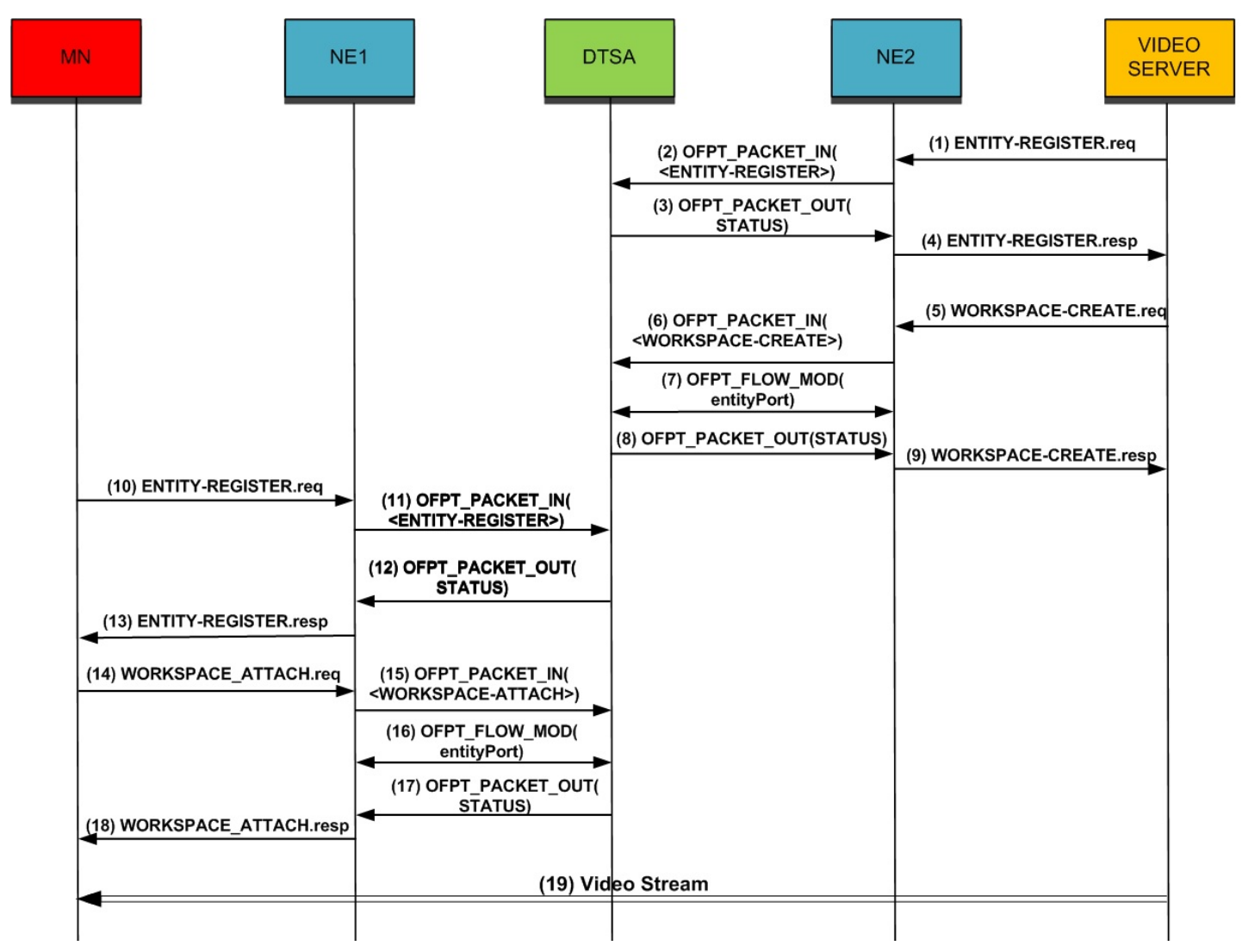

Figura 27: Inicialização do Cenário utilizando protocolo ETCP

indicação cria um novo Workspace, o identifica por seu título e cria uma regra associada. Utilizando uma mensagem OFPT_FLOW_MOD do protocolo OpenFlow esta regra é adicionada à flow table dos switches envolvidos (neste caso o NE1 e o NE2).

A seguir uma entidade já registrada, nomeada neste cenário como Mobile Node $(\mathrm{MN})$, deseja receber a mídia fornecida pelo Workspace e para tanto envia uma primitiva WORKSPACE_ATTACH.req. Esta primitiva por sua vez será encaminhada ao DTSA através do uso de uma mensagem OFPT_PACKET_IN. Através de um OFPT_FLOW_MOD a flow table será modificada a fim de incluir a porta física onde a entidade que requisita (MN) está conectada neste momento. Ao final do processo de anexação, a entidade poderá receber a media fornecida pelo Workspace. Neste ponto, o fluxo de vídeo (mensagem 19 na Figura 27) representa a mídia que está sendo consumida 
pela entidade.

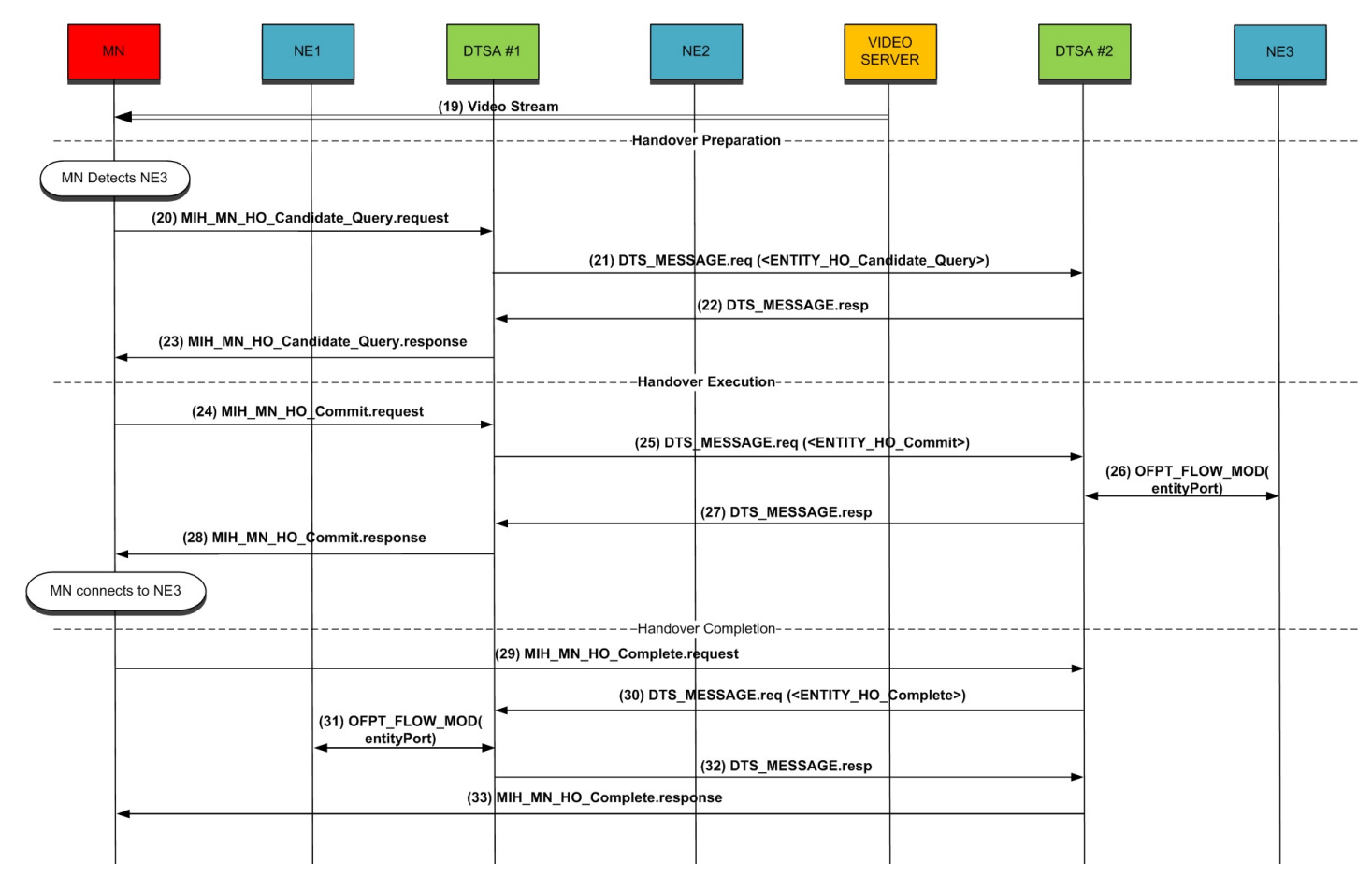

Figura 28: Handover Assistido pelo Mobile Node e Controlado pela Rede, entre Dois Diferentes DTSAs

Considerando que o MN ao movimentar-se detecta uma rede com melhor qualidade (de sinal por exemplo), o mesmo decide então iniciar um processo de handover a fim de receber o vídeo a partir desta nova rede (neste caso a rede em que está conectado o NE3). O MN inicia o processo utilizando o protocolo MIH e envia uma mensagem MIH_MN_HO_Candidate_Query.request para o DTSA onde está registrado neste momento. O DTSA\#1 ao receber esta mensagem, verifica que o NE3 não está em seu domínio e portanto envia uma primitiva DTS_MESSAGE.req(ENTITY_HO_Candidate_Query) para o DTSA que controla o NE3. Ao receber esta mensagem do DTSA\#2 verifica internamente se possui os recursos necessários a fim de acomodar o MN e os Workspaces a ele associados e envia a resposta ao DSTA\#1 que por sua vez encaminha o resultado ao MN (mensagem MIH_Net_HO_Candidate_Query.response).

Neste ponto o MN verifica se é viável mover-se para o NE3 e, conside- 
rando isto possível, notifica o DSTA\#1 sobre a rede de destino selecionada, enviando uma mensagem MIH_MN_HO_Commit.request, que então será encaminhada ao DTSA\#2 através de uma primitiva DTS_MESSAGE.req (ENTITY_HO_Commit). O DTSA\#2, neste momento, registra a entidade e realizada sua anexação ao Worskpace. Antes de realizar este processo, pode ser necessário efetuar um WORKSPACE-LOOKUP a fim de adicionar o NE3 ao Workspace. Quando os recursos estão garantidos, o DTS\#2 informa ao MN, através do DTSA\#1, que os recursos estão reservados e que o MN pode então se mover para a nova rede. Ao receber esta mensagem, o MN pode então conectar-se no NE3 e começar a receber a mídia através do Workspace. Deste momento em diante, o MN pertence ao DTSA\#2 e, portanto, este passa a ser o seu novo Point of Service (PoS) e o NE3 o seu PoA.

Após o processo de handover, o MN informa ao DTSA\#2 o seu resultado, enviando um mensagem $M I H \_M N \_H O \_C o m p l e t e . r e q u e s t . \quad \mathrm{O}$ DTSA\#2 encaminha esta mensagem ao DTSA\#1, através de uma primitiva DTS_MESSAGE.req(ENTITY_HO_Complete), com o objetivo de informá-lo que o MN não está mais no seu domínio. Então o DSTA\#1 pode requisitar ao NE1 que libere os recursos e subscrições associadas com o MN utilizando para isto uma mensagem OFPT_FLOW_MOD(entityPort).

\subsubsection{Handover de Entidades}

Esta seção apresenta um cenário de avaliação da arquitetura ETArch considerando sua implementação final, apresentada na Seção 4.1. O objetivo desta avaliação é realizar testes da comunicação baseada sobre o Workspace e verificar como seria o comportamento durante o handover de uma entidade. Este experimento permitiu verificar a perda de pacotes e o custo de sinalização de controle associada ao processo de handover. O experimento 
considerou duas diferentes situações: uma em que o handover ocorre apenas através do Workspace e a outra, onde o protocolo IEEE 802.21 foi utilizado a fim de permitir a mobilidade transparente através do Worspace. Outro objetivo é realizar o experimento sobre uma infraestrutura real, baseada em hardware.

A avaliação experimental foi realizada na ilha OFELIA que foi implantada no Brasil, mais especificamente em Uberlândia, dentro da proposta EDOBRA (ATNOG, 2012). Como mostrado na Figura 29, o cenário avaliado consiste de dois diferentes PoAs (modelo TP-Link TL-WR1043ND) que podem ser controlados tanto via OpenFlow quanto através do IEEE 802.21. Esses dois PoAs estão conectados a um switch OpenFlow (Datacom DM4000 ETH24GX+2x10GX). O DTSA contém os componentes conforme mostrado na Figura 24 e está conectado aos dispositivos OpenFlow através de duas conexões físicas: uma para controle e outra para dados. Os dispositivos Móveis (MN1, MN2), e o Video Server completam o cenário de avaliação.

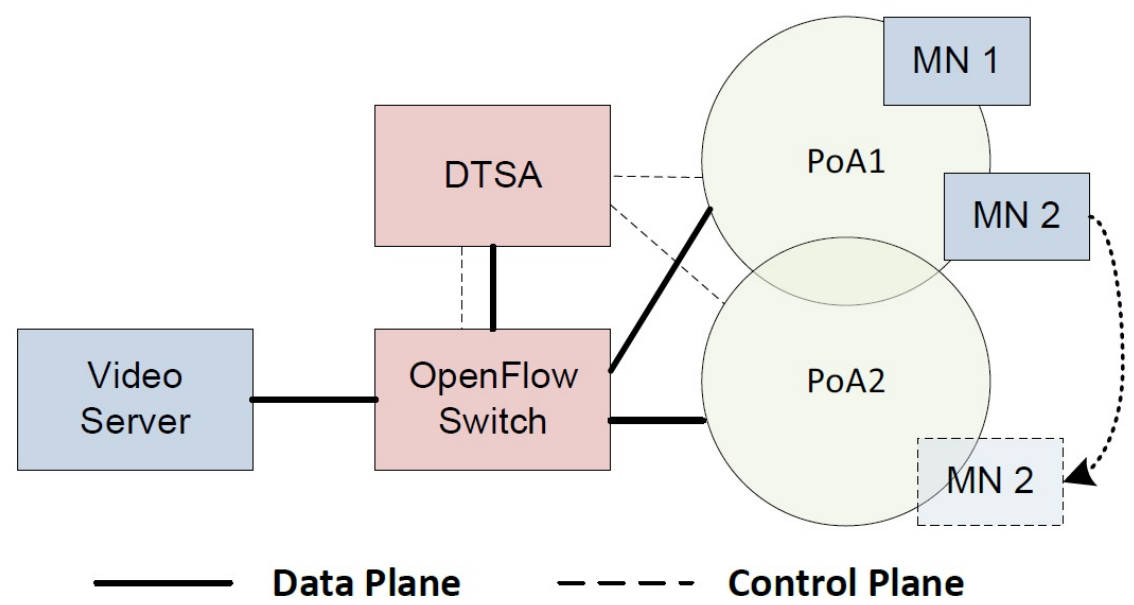

Figura 29: Cenário de Avaliação do Handover de uma entidade (MN).

O Video Server contém uma aplicação que envia uma stream de vídeo no formato H.264 sobre um workspace, cujo Título é "edobra". MN1 e MN2 estão anexados a este Workspace. Assim as primitivas ETCP mostradas na Figura 27 foram trocadas entre essas entidades e o DTSA. Nesse momento, tanto 
MN1 quanto MN2 são capazes de receber o vídeo, através do PoA1.

No experimento realizado, o MN2 se movimenta do PoA1 para o PoA2, enquanto continua a receber o vídeo a partir do Video Server. Durante a movimentação o MN2 detecta uma queda no sinal do PoA1. A partir desse momento, inicia-se o processo de handover utilizando uma sinalização de controle, semelhante à mostrada na Figura 28 . Em sua execução, o DTSA é o responsável por atualizar o workspace em direção ao PoA2. Então o MN2 executa o handover e, neste momento, recebe dados por ambas as interfaces de rede. Após este ponto, o MN2 se desconecta do PoA1 e indica ao DSTA que o handover foi completado. Ao final do processo o DTSA pode remover o workspace do PoA1. Deve ser ressaltado que esta remoção não ocorre neste experimento visto que o MN1 ainda está anexado ao workspace através do PoA1.

O handover do MN2 para o PoA2 foi iniciado sempre a dez segundos após o inicio do experimento a fim de correlacionar os resultados. Cada experimento foi executado dez vezes, sendo que os valores médios foram calculados com um intervalo de confiança de $95 \%$ utilizando T-Student.

A Figura 30 mostra uma comparação visual do mesmo instante do vídeo nas duas situações propostas pelo experimento. Na primeira delas (Figura 30(a)) é apresentado o efeito visual que ocorre durante o handover da entidade sem o uso dos mecanismos de mobilidade implementados pelo IEEE 802.21. Por outro lado, ao utilizar esses mecanismos (conforme sinalização apresentada na Seção 4.2.2) durante o handover não é possível perceber alterações visuais no vídeo, o que pode ser percebido pela Figura 30(b).

A Figura 31 mostra quatro diferentes terminais durante a execução do experimento. Os dois gráficos superiores mostram o tráfego de pacotes por cada uma das interfaces do MN2. Neste caso o tráfego inicia-se por uma interface 


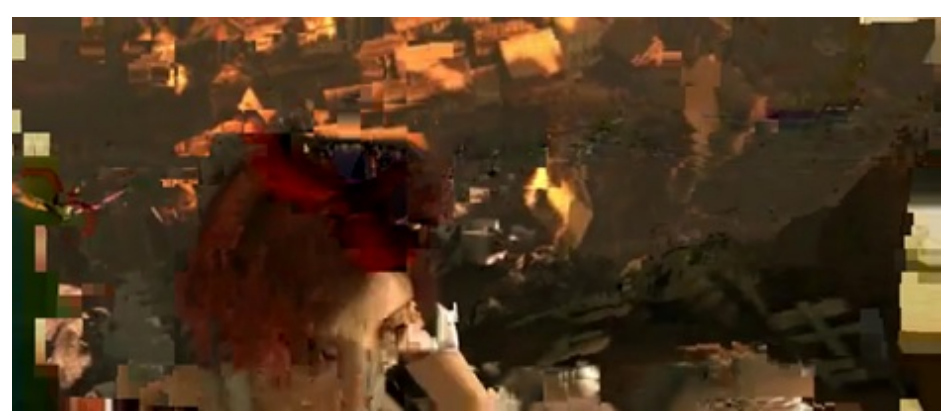

(a) Mobilidade no Workspace com uso do IEEE 802.21.

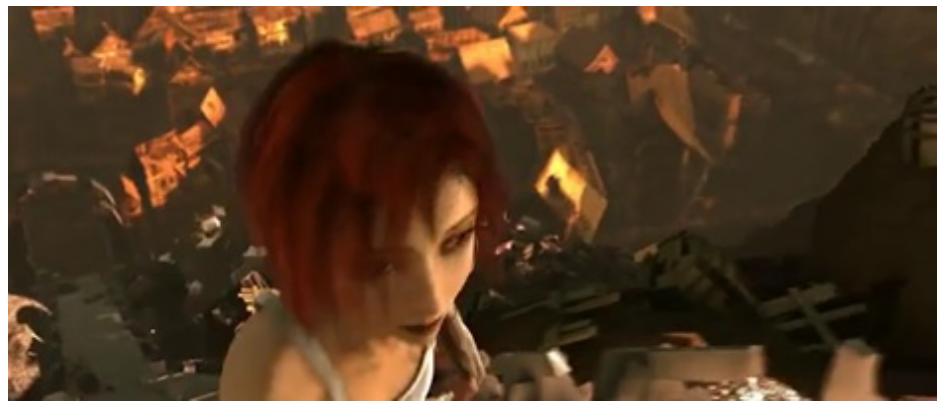

(b) Somente uso do Workspace.

Figura 30: Cena do Vídeo durante Handover de Entidade (MN2) anexada ao Workspace.

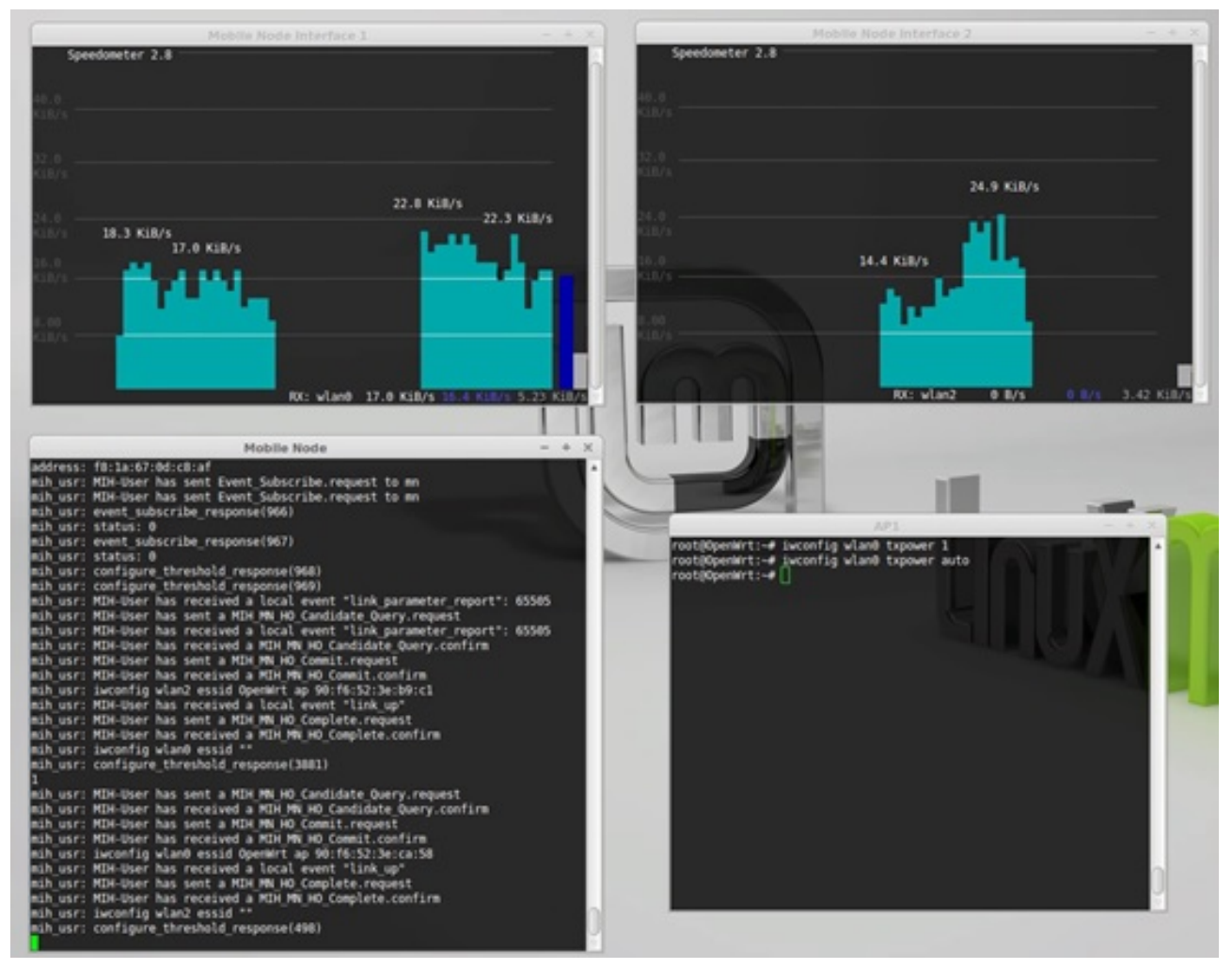

Figura 31: Tela com Tráfego das Interfaces de Rede do MN2 durante Handover. 
(mostrada no canto superior esquerdo) e após o handover o tráfego continua pela outra interface (mostrada no canto superior direito da figura) e finalmente o tráfego volta para a primeira interface, indicando neste caso o retorno ao MN2 à posição inicial, ou seja, PoA1. O terminal no canto inferior esquerdo apresenta as mensagens do protocolo $\mathrm{MIH}$ trocadas durante o processo entre o DTSA e o MN2. O terminal do canto inferior direito por sua vez mostra o acesso ao PoA1 e o recurso utilizado para simular a baixa do sinal deste equipamento (comando iwconfig com uso da opção txpower), o que equivaleria à movimentação do MN2.

A Figura 32 apresenta a recepção de conteúdo no MN2 (em Bytes a cada $100 \mathrm{~ms}$ ) durante o experimento, apresentando tanto a abordagem apenas baseada no Workspace quanto a outra que emprega os mecanismos de mobilidade do IEEE 802.21.

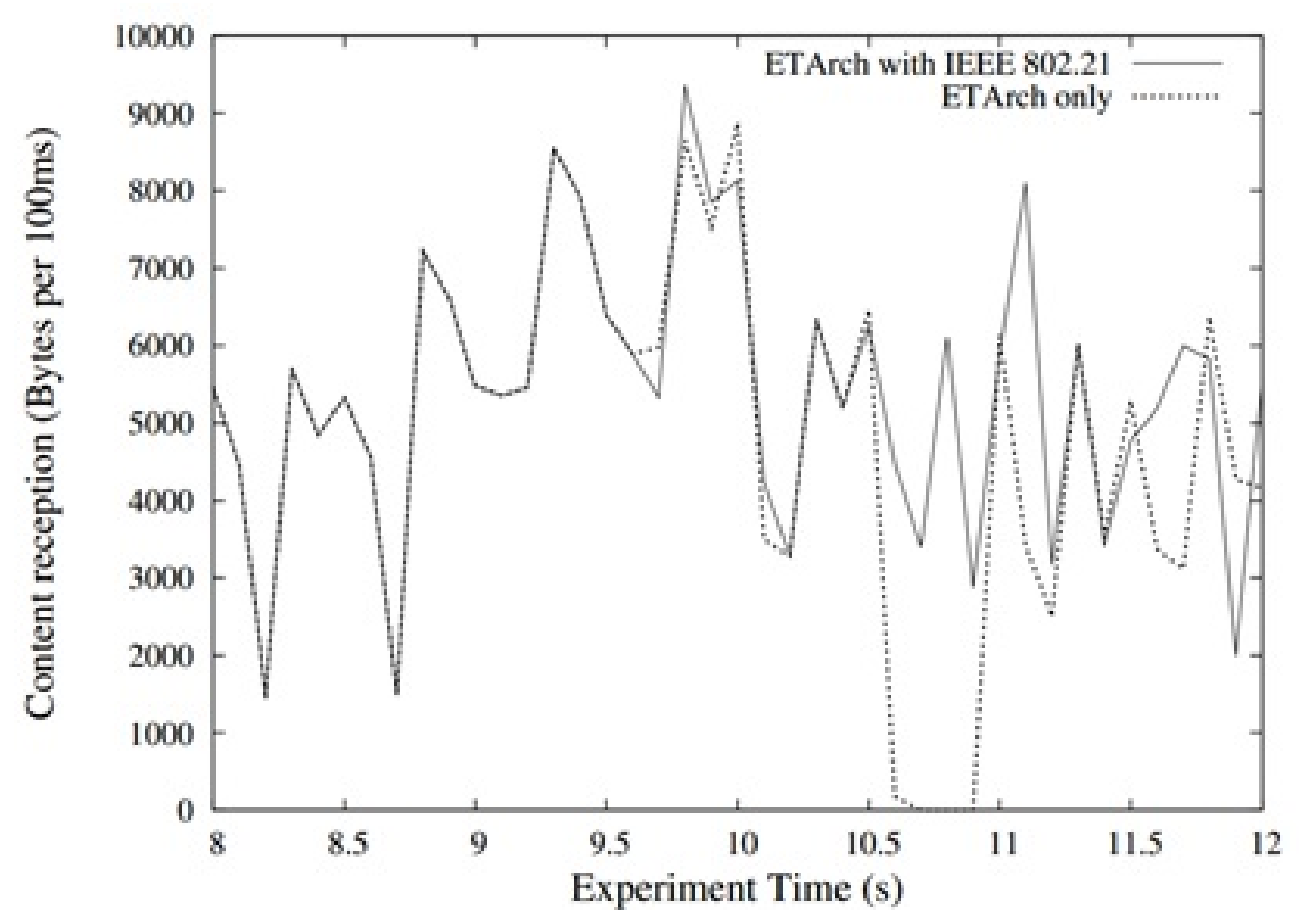

Figura 32: Recepção de Conteúdo no MN2 ao Longo do Tempo de Experimento.

A partir dos experimentos foi obtida Tabela 8 que apresenta alguns parâ- 
metros que foram investigados durante o handover. Assim foi possível comparar o impacto na recepção do conteúdo pelo MN2 em um cenário onde os mecanismos de mobilidade são utilizados com o outro, onde apenas foi utilizado o Workspace sem o uso do IEEE 802.21. A tabela mostra a duração e o número de pacotes duplicados (tanto na rede como no MN2), os pacotes perdidos durante o handover e o tempo necessário para o restabelecimento da stream após o handover.

Tabela 8: Impacto do Handover sobre a Transmissão do Conteúdo Através do Workspace.

\begin{tabular}{lcc}
\hline & $\begin{array}{c}\text { ETArch with } \\
\text { IEEE 802.21 }\end{array}$ & ETArch only \\
\hline \hline Lost packets during HO & $\approx 0$ & $19.4 \pm 1.0$ \\
\hline Restore stream delay (ms) & $\approx 0$ & $407.9 \pm 26.9$ \\
\hline Redundancy at MN (ms) & $40.3 \pm 12.4$ & 0 \\
\hline Redundancy at network (ms) & $851.7 \pm 32.1$ & 0 \\
\hline Redundancy at network (packets) & $39.6 \pm 3.1$ & 0 \\
\hline
\end{tabular}

A Tabela 8 indica que no cenário em que o handover ocorre de forma transparente, utilizando do protocolo IEEE 802.21, não há perda de pacotes. Isto decorre do fato de que o MN2 apenas de desconecta do PoA1 quando o Workspace já engloba o PoA2 e no momento em que a conexão com o PoA2 está plenamente estabelecida. Dessa forma, durante o processo de handover, por $40.3 \pm 12.4 \mathrm{~ms}$, o MN2 recebe conteúdo por ambas as interfaces. Além disso, considerando que o Workspace já foi estendido não há atraso para a restauração da stream, entretanto isto leva a uma redundância na rede durante um período de $851.7 \pm 32.1 \mathrm{~ms}$, o que representa aproximadamente 40 quadros.

No cenário em que é utilizado apenas o Workspace, após o processo de handover que levou cerca de 779 ms, MN2 deve se desconectar do DTSA através do PoA1 e então proceder com o um novo registro através do PoA2. 
O procedimento de registro levou $407.9 \pm 26.9 \mathrm{~ms}$, e durante este periodo, o MN2 não recebeu nenhum quadro (conforme pode ser visto na Figura 32) o que corresponde a $19.4 \pm 1.0$ quadros perdidos. Em consequência, neste caso não há redundância de pacotes, em oposto ao que ocorre quando o IEEE 802.21 é utilizado a fim de permitir a mobilidade transparente.

Tabela 9: Sinalização de Controle de Cada Protocolo Envolvido.

\begin{tabular}{llccc}
\hline & & HO Preparation & HO Commit & HO Complete \\
\hline \hline \multirow{3}{*}{$\begin{array}{l}\text { Size } \\
\text { (bytes) }\end{array}$} & IEEE 802.21 & 235 & 171 & 218 \\
\cline { 2 - 5 } & OpenFlow & 0 & 2568 & 0 \\
\cline { 2 - 5 } & ETCP & 0 & 0 & 0 \\
\hline \multicolumn{2}{c}{ Time (ms) } & $108.3 \pm 18.5$ & $65.7 \pm 17.5$ & $32.0 \pm 10.9$ \\
\hline
\end{tabular}

Outro aspecto avaliado foi o custo associado (em termos de bytes) devido exclusivamente à sinalização de controle. A Tabela 9 apresenta a sinalização envolvida no processo de handover que é auxiliado pelo protocolo IEEE 802.21. Sendo assim é possível observar a sinalização associada a cada protocolo de controle envolvido (IEEE 802.21, OpenFlow e ETCP) durante o procedimento de handover.

A Tabela 9 indica que cerca de $20 \%$ da sinalização utilizada no processo de handover corresponde a primitivas do protocolo MIH (IEEE 802.21), enquanto os $80 \%$ restantes corresponde ao protocolo OpenFlow. Entretanto é importante notar que a sinalização IEEE 802.21 não é dependente do número de workspaces, o que não ocorre com o protocolo OpenFlow. Deve ser notado que em relação ao protocolo ETCP não houve nenhuma sinalização de controle, já que as operações necessárias foram disparadas pelo protocolo IEEE 802.21 .

Em termos de tempo, a fase de preparação para o handover (HO Preparation) foi a de maior duração $(108.3 \pm 18.5$ ms $)$. Este é o tempo necessário 
a fim de calcular os recursos necessários em cada PoA, a fim de encontrar o melhor candidato para o MN. No cenário experimental realizado a fase de commit (HO Complete) foi a de menor duração já que o MN1 continuou anexado no PoA1 não sendo necessário remover o Workspace deste dispositivo. Ainda, a sobrecarga em termos de tempo introduzida pelos procedimentos do IEEE 802.21 representam $21 \%$ do tempo total de handover. Os $79 \%$ restantes são decorrentes do procedimento de handover de L2 que levou $779.9 \pm$ 29.3 ms. O procedimento de handover de L2 envolve a busca, associação e autenticação do MN2 ao PoA2.

\subsection{Análise de Resultados}

Este trabalho parte do pressuposto de que a arquitetura das redes baseadas na comutação de pacotes, em especial a Internet, necessita ser revista e que este processo inicia-se pela questão do endereçamento e da identificação das entidades que se comunicam.

Partindo de um modelo (PEREIRA et al., 2011), o trabalho resulta em uma arquitetura de rede, chamada ETArch, capaz de realizar os conceitos estabelecidos, em especial o Workspace, o Title e o DTS (SILVA et al., 2011), formado por um conjunto de DTSA interconectados. Utilizando o conceito de SDN, a arquitetura foi construída sobre um raciocínio que explora a abstração do controle tanto de enlaces com fio, através do protocolo OpenFlow (SILVA et al., 2012), quanto de enlaces sem fio, através do protocolo MIH (GUIMARãES et al., 2013). O uso dessas tecnologias permite que a ETArch possa ser implantada com os recursos atualmente disponíveis, especialmente em ambientes controlados, como a rede de uma operadora de telecomunicações.

A visão abstrata de uma entidade e uma identificação neutra, seu Título, 
independente da topologia, permite que a arquitetura possa adaptar-se a diferentes cenários e aplicações. Isto permite que a ETArch possa adequar-se a diferentes visões da Internet do Futuro, seja ela baseada em serviços, usuários, hosts, coisas (things) ou conteúdo.

Tabela 10: Comparação entre Arquiteturas de Internet do Futuro

\begin{tabular}{|c|c|c|c|}
\hline \multirow{2}{*}{ Critério } & \multicolumn{3}{|c|}{ Arquiteturas de Internet do Futuro } \\
\hline & XIA & Mobility First & ETArch \\
\hline Identificação & Principal (XID) & GUID & Title \\
\hline Endereço & DAG & Endereço Rede & PoA \\
\hline Mapeamento entre Identificação e Endereço & XARP & GNRS & DTSA \\
\hline Nova Pilha de Protocols & Sim & Sim & Sim \\
\hline Entrega multipath & Sim & Sim & Não \\
\hline Multicast & $\begin{array}{l}\text { Baseado em um } \\
\text { tipo espefício de } \\
\text { Principal }\end{array}$ & $\begin{array}{l}\text { Baseado em } \\
\text { Grupo Multicast }\end{array}$ & Nativo \\
\hline Unicast & Baseado no XID & Baseado em GUID & Nativo \\
\hline Roteamento Adaptativo & XIP & GSTAR & Workspace \\
\hline Uso de Informação de Roteamento & Hop-a-Hop & Hop-a-Hop & $\begin{array}{l}\text { Atualização } \\
\text { Workspace }\end{array}$ \\
\hline Mobilidade & Nativo & Nativo & Nativo \\
\hline
\end{tabular}

A Tabela 10 estende a comparação qualitativa apresentada na seção 2.3 .3 e apresenta um quadro comparativo entre as arquiteturas XIA (apresentada na seção 2.3.2.2), Mobility First (apresentada na seção 2.3.2.1) e ETArch.

A partir dos critérios utilizados, é possível realizar uma comparação entre as mesmas. É necessário, porém, destacar que não se encontra uma correspondência em relação ao conceito do Workspace, utilizado pela ETArch. Na ETArch existe uma diferença em relação ao uso da informação de roteamento que no caso de ambas é feita em cada nó, por onde um pacote trafega, enquanto na ETArch, esta pesquisa não é necessária, considerando que no caso do Workspace isto ocorre out-of-band, através do plano de controle, sendo coordenado por cada DTSA ao longo do Workspace.

Um aspecto apresentado neste trabalho foi a capacidade nativa do Workspace realizar o multicast das primitivas, sendo este o modo padrão de encaminhamento oferecido pela arquitetura. A avaliação experimental, apresentada na Seção 4.2.1, mostrou que o consumo da banda de comunicação na ori- 
gem permanece constante não importando o número de clientes conectados ao Workspace. Além disso, este consumo da banda de dados permanece constante ao longo da rede. Deve ser ressaltado que o comparativo levou em conta o modo padrão de encaminhamento existente e amplamente utilizado na arquitetura TCP/IP (SILVA et al., 2012b).

O consumo de banda de comunicação pelo Workspace, além de ser mais eficiente do ponto de vista energético, sugere que os enlaces atuais podem ser utilizados para usuários cada vez mais ávidos pelo aumento da vazão e por transmissões de vídeo em ultra alta definição (4K e 8K).

O Conceito do Workspace e o endereçamento projetados neste trabalho permitem também acomodar outros requisitos das futuras redes de computadores como mobilidade, segurança e QoS.

A sinalização de controle mostrada no cenário de mobilidade entre DTSAs, descrito na Seção 4.2.2, indica a atuação dos protocolos de controle da arquitetura ETCP e DTSCP e sua integração com os protocolos OpenFlow e MIH (SILVA et al., 2013). Além disso, os protocolos apresentam em sua interface a possibilidade de acomodar a expressão dos requisitos e capacidades das entidades, assim como do Workspace conforme detalhado nas Seções C.2.1 e C.2.2 respectivamente.

O experimento apresentado na Seção 4.2.3, realizado sobre uma infraestrutura física do projeto OFELIA, mostrou a mobilidade de entidades sobre o workspace juntamente com o uso dos mecanismos previstos na especificação IEEE 802.21. Desta forma foi possível a mobilidade transparente, sem perdas de primitivas e com um custo de sinalização aceitável quando comparado com o cenário que não utiliza tais mecanismos.

A abordagem utilizada na ETArch permite que o workspace seja reconfi- 
gurado de forma dinâmica através da escolha antecipada do PoA mais adequado para o handover, levando em conta as condições percebidas pelo MN, evitando assim impactos nas sessões estabelecidas no MN. Pela Figura 30 visualmente é possível perceber que a QoE não é afetada durante o procedimento de handover. Deve ser destacado que a abordagem baseada no IEEE 802.21 pode ser aplicada a outras tecnologias de acesso, permitindo que novos cenários de uso envolvendo dispositivos móveis e a mobilidade entre estas diferentes redes de acesso possam ser explorados.

É importante citar dois resultados que estão diretamente ligados a este trabalho, pois mostram que os conceitos desenvolvidos na arquitetura ETArch são corroborados pela comunidade científica nacional e internacional, envolvida na pequisa arquiteturas para a próxima geração de redes de computadores.

Em 2012, o projeto OFELIA (OFELIA, 2011) publicou uma chamada aberta à comunidade científica (OFELIA, 2012a), visando agregar novos parceiros ao projeto. A partir de resultados já existentes deste trabalho, uma arquitetura inicial criada em um dos ciclos de desenvolvimento e de uma visão de integração com mecanismos de mobilidade, foi submetida a proposta chamada Extending and Deploying Ofelia in BRAzil (EDOBRA) (ATNOG, 2012), que além do desenvolvimento e experimentação da arquitetura ETArch visava ampliar a extensão física do OFELIA, através de uma implantação de uma ilha em Uberlândia(MG), que está interligada às outras ilhas (OFELIA, 2012b) na Europa.

A proposta EDOBRA foi uma das escolhidas (OFELIA, 2012c), possibilitando assim ampliar o trabalho realizado com a ETArch, e a construção de uma colaboração internacional a Escola Politécnica da Universidade de São Paulo, o Instituto de Telecomunicações, da Universidade de Aveiro em Portu- 
gal, e a Faculdade de Computação da Universidade Federal de Uberlândia.

A partir deste trabalho de pesquisa, a Universidade Federal de Uberlândia foi convidada a associar-se ao projeto FIBRE (FIBRE, 2013b) que conta com financiamento conjunto Brasil (CNPq) e Comissão Europeia (FP7). Como resultado, na Faculdade de Computação da Universidade Federal de Uberlândia, no mesmo espaço onde está hospedada a ilha do OFELIA, está em implantação uma ilha do FIBRE. Esta ilha estará interligada tanto com a Europa como com diversas outras instituições no Brasil. 


\section{CONCLUSÃO E TRABALHOS FUTUROS}

O objetivo deste trabalho foi definir, projetar e avaliar experimentalmente uma forma de encaminhamento de primitivas que possa ser utilizada em cenários onde o multicast seja um requisito central para as aplicações, propiciando uma utilização eficiente de recursos. Além disso, esta forma de encaminhamento deveria ser baseada em novas relações entre as entidades que se comunicam e na utilização de uma identificação única, não ambígua e independente da topologia: seu Título.

O argumento básico é que a revisão da arquitetura das redes de computadores, inicia-se pelo endereçamento. A alteração do endereçamento e também na identificação das entidades remete a uma nova arquitetura.

O IPv6 também adota a estratégia de um novo endereçamento, porém, mesmo com a sua implantação, requisitos como multicast, mobilidade, segurança e QoS não serão satisfeitos. Dessa forma, o trabalho utiliza uma abordagem Clean Slate a fim de projetar uma arquitetura para a próxima geração das redes de computadores. É importante destacar que o presente trabalho é oportuno e está em consonância com a busca realizada pela comunidade científica internacional e também nacional, pela Internet do Futuro. O trabaIho representa uma contribuição genuinamente Brasileira para esta área de pesquisa.

O trabalho definiu e projetou uma nova forma de identificação e um novo 
esquema de endereçamento capazes de distinguir de maneira precisa as entidades envolvidas na comunicação. Esta identificação e endereçamento baseados no Título favorecem a mobilidade e ainda podem acomodar futuramente, por exemplo, requisitos de segurança como a autenticação de entidades.

A definição do Workspace, implementado neste trabalho, permite estabelecer um canal por onde múltiplas entidades se comunicam naturalmente de uma forma multicast, permitindo ainda a mobilidade dessas entidades ao longo dos NEs que as suportam. Identificado por seu Título, o Workspace é o destino das primitivas durante a comunicação. À medida que novas entidades se ligam ou desligam, há reconfiguração do Workspace, podendo ainda alterar suas capacidades e requisitos ao longo do tempo. A abordagem definida, implementada e verificada neste trabalho representa uma forma mais eficiente de comunicação se comparada aos endereços no âmbito da camada de rede (IPMulticast) ou de aplicação (ALM).

A arquitetura ETArch estabelece novas relações entre as entidades de sistemas distribuídos. O comportamento dessas novas relações é definido pelos protocolos ETCP, que trata da relação de uma entidade com o DTSA e o protocolo DTSCP, que trata da relação entre as entidades que compõem o DTS, ou seja, os DTSAs. Os protocolos ETCP e DTSCP são os responsáveis pelo plano de controle. A arquitetura ETArch leva em conta não somente os aspectos de multicast e mobilidade das entidades, como também foi construída de uma forma modular permitindo incorporar em momento posterior, por exemplo, requisitos de QoS e segurança.

Construída sobre uma infraestrutura baseada em redes definidas por software, a arquitetura leva em conta a abstração do controle tanto de enlaces cabeados, através do protocolo OpenFlow, quanto de enlaces sem fio, através do protocolo MIH. Através desta abstração, o DTSA assume controle do 
enlace. A implementação dos componentes permitiu a experimentação dos protocolos e a avaliação dos conceitos. Além disso, a arquitetura do DTSA com módulos desacoplados permite sua evolução a fim de tratar novos requisitos da próxima geração de redes de computadores.

Dentre os componentes implementados, alguns podem ser utilizados por uma gama maior de desenvolvedores e pesquisadores, como os Resource Adaptors, tanto para OpenFlow quanto para o $\mathrm{MIH}$. A abordagem proposta, baseada em redes definidas por software, permite uma visão cross layer das camadas da ETArch e, além disso, permite experimentação, no momento presente, utilizando tecnologias disponíveis.

Existem algumas direções claras de pesquisas que poderão ser realizadas a partir deste trabalho, sendo que algumas delas já encontram-se em andamento, representando assim, frutos deste trabalho que resultarão em futuros mestres e doutores.

Um ponto relevante da arquitetura ETArch, mesmo em relação a outras arquiteturas de redes, como, por exemplo, as citadas na seção 2.3.2, é a capacidade de expressar requisitos e capacidades do domínio de comunicação de entidades: o Worskpace. Dessa forma, a arquitetura ETArch possui em sua concepção a visão de que a rede deve ser capaz de suportar os requisitos das aplicações e por sua vez coordenar os equipamentos de rede a fim de que estes requisitos sejam traduzidos conforme suas potencialidades.

O primeiro trabalho que surge em relação a este tema é especificar e desenvolver mecanismos que permita às aplicações expressar diferentes requisitos em especial os ligados à mobilidade, QoS e Segurança, otimizando a operacionalização desses aspectos nas camadas da rede, propiciando também ajustes dinâmicos ao longo do tempo. Dessa forma uma classe de aplicações poderá ser construída sobre a ETArch, permitindo explorar seu potencial. 
O segundo trabalho consiste em desenvolver mecanismos que garantam QoS baseados em requisitos definidos por aplicações. Esses mecanismos poderiam ser integrados à arquitetura do DTSA a fim de suportar, por exemplo, aplicações multimídia móveis com Qualidade de Serviço garantida durante todo seu ciclo de vida. Esta linha já está sendo explorada por alunos do Programa de Pós-Graduação em Sistemas e Computação da Universidade Federal do Rio Grande do Norte.

Outro aspecto fundamental para a próxima geração de redes de computadores é a segurança. A arquitetura ETArch, o Workspace e a identificação não ambígua das entidades podem suportar uma nova abordagem para a segurança. O Título pode ser utilizado para auxiliar no aspecto de autenticação, sendo que o processo de registro de entidades poderia também garantir aspectos de autorização. Além disso, a confidencialidade poderia ser uma capacidade associada ao Workspace.

Uma outra área que poderia ser alvo de futuras pesquisas é um DTSA tolerante à falhas stateful. A arquitetura do DTSA proposta, baseada do modelo de componente do JAIN SLEE poderia ser a base para uma investigação futura neste tópico. Além disso, outra possibilidade é a integração do DTSA com outros protocolos de serviço comumente utilizados em plataformas de telecomunicações, permitindo assim investigar a integração do DTSA com outras entidades existentes nas redes das operadoras de telecomunicações.

Um outro trabalho que poderá ser explorado é a implantação da arquitetura ETArch a fim de criar uma rede de propósito específico dentro de um ambiente controlado, como uma operadora de telecomunicações. Isto poderia viabilizar novos serviços para a operadora, permitindo assim que o resultado desta pesquisa possa ser refletido no dia-a-dia da sociedade.

Finalmente uma outra área que poderia ser explorada é um estudo cujo 
objetivo seria o desenvolvimento de switch que poderia ser integrado tanto às redes atuais, através do suporte ao OpenFlow, quanto à arquitetura ETArch, possuindo em hardware, parte das funcionalidades que hoje estão a cargo do DTSA.

Pelo exposto, uma certeza é que este trabalho não representa um fim, mas um ponto de partida para várias outras iniciativas de pesquisas que poderão ser conduzidas no âmbito das colaborações nacionais e internacionais visando dialogar com diferentes grupos a fim de construir a próxima geração das redes de computadores. 


\section{REFERÊNCIAS}

ABELEM, A. et al. Advances in developing a Future Internet testbed in Brazil. jul. 2011. Disponível em: <http://www.ic.uff.br/ michael/FIBRE-position-paper.v2.pdf>. Acesso em: 07 jul. 2011.

ABLEY, J.; SAVOLA, P.; NEVILLE-NEIL, G. Deprecation of Type 0 Routing Headers in IPv6. IETF, 2007. (Request for Comments, 5095). Published: RFC 5095 (Proposed Standard). Disponível em: <http://www.ietf.org/rfc/rfc5095.txt>.

AGUIAR, R. L. Some comments on hourglasses. SIGCOMM Comput. Commun. Rev., v. 38, n. 5, p. 69-72, set. 2008. ISSN 0146-4833. Disponível em: <http://doi.acm.org/10.1145/1452335.1452346>.

AGUIAR, R. L. et al. ODTONE - Open Dot Twenty One. 2011. Http://atnog.av.it.pt/odtone/. Disponível em: <http://atnog.av.it.pt/odtone/>.

Al-Fares, M. et al. Hedera: dynamic flow scheduling for data center networks. In: Proceedings of the 7th USENIX conference on Networked systems design and implementation. Berkeley, CA, USA: USENIX Association, 2010. (NSDl'10), p. 19-19. Disponível em: <http://dl.acm.org/citation.cfm? $\mathrm{id}=1855711.1855730$ > .

AL-SURMI, I.; OTHMAN, M.; ALI, B. M. Mobility management for IP-based next generation mobile networks: Review, challenge and perspective. Journal of Network and Computer Applications, v. 35, n. 1, p. 295-315, jan. 2012. ISSN 1084-8045. Disponível em: <http://www.sciencedirect.com/sciencelarticle/pii/S1084804511001792>.

AMANTE, S. et al. IPv6 Flow Label Specification. IETF, 2011. (Request for Comments, 6437). Published: RFC 6437 (Proposed Standard). Disponível em: <http://www.ietf.org/rfc/rfc6437.txt>.

AMBROSCH, W.-D.; MAHER, A.; SASSCER, B. The intelligent network. In: AMBROSCH, W.-D.; MAHER, A.; SASSCER, B. (Ed.). The Intelligent Network. Berlin, Heidelberg: Springer Berlin Heidelberg, 1989. p. 5-21. ISBN 978-3-540-50897-7, 978-3-642-46663-2. Disponível em: <http://link.springer.com/content/pdf/10.1007/978-3-642-46663-2_2.pdf >.

ARKKO, J.; BRADNER, S. IANA Allocation Guidelines for the IPv6 Routing Header. IETF, 2010. (Request for Comments, 5871). Published: RFC 5871 (Proposed Standard). Disponível em: <http://www.ietf.org/rfc/rfc5871.txt>. 
ARKKO, J.; KERANEN, A. Experiences from an IPv6-Only Network. IETF, 2012. (Request for Comments, 6586). Published: RFC 6586 (Informational). Disponivel em: <http://www.ietf.org/rfc/rfc6586.txt>.

ATNOG. EDOBRA (Extending and Deploying OFELIA in BRAzil). 2012. Disponível em: <http://atnog.av.it.pt/content/ofelia-edobra>.

ATZORI, L.; IERA, A.; MORABITO, G. The internet of things: A survey. Computer Networks, v. 54 , n. 15 , p. 2787-2805, out. 2010. ISSN 1389-1286. Disponível em: <http://www.sciencedirect.com/science/article/pii/S1389128610001568>.

AURA, T. Mobile IPv6 security. In: CHRISTIANSON, B. et al. (Ed.). Security Protocols. Springer Berlin Heidelberg, 2004, (Lecture Notes in Computer Science, 2845). p. 215-234. ISBN 978-3-540-20830-3, 978-3-540-39871-4. Disponível em: <http://link.springer.com/chapter/10.1007/978-3-540-39871-4_18>.

BALADRóN, C. et al. User-centric future internet and telecommunication services. In: Towards the Future Internet. [s.n.], 2009. p. 217-226. ISBN 978-1-60750-431-3. Disponível em: <http://ebooks.iospress.nl/publication/29787>.

BAO, C. et al. IPv6 Addressing of IPv4/IPv6 Translators. IETF, 2010. (Request for Comments, 6052). Published: RFC 6052 (Proposed Standard). Disponível em: <http://www.ietf.org/rfc/rfc6052.txt>.

BARAN, P. On distributed communications networks. IEEE Transactions on Communications Systems, v. 12, n. 1, p. 1-9, mar. 1964. ISSN 0096-1965.

BASS, L.; CLEMENTS, P.; KAZMAN, R. Software Architecture in Practice. 2. ed. [S.I.]: Addison-Wesley Professional, 2003. ISBN 0321154959.

BIG SWITCH. Developing Floodlight Modules. Floodlight OpenFlow Controller. jan. 2012. Http://floodlight.openflowhub.org/developing-floodlight/. Disponível em: <http://floodlight.openflowhub.org/developing-floodlight/>.

. Floodlight OpenFlow Controller. jan. 2012.

Http://floodlight.openflowhub.org/. Disponível em: <http://floodlight.openflowhub.org/>.

BUSCHMANN, F. et al. Pattern-Oriented Software Architecture Volume 1: A System of Patterns. Volume 1. [S.I.]: Wiley, 1996. ISBN 0471958697.

CAICEDO, C.; JOSHI, J.; TULADHAR, S. IPv6 security challenges. Computer, v. 42, n. 2, p. 36-42, 2009. ISSN 0018-9162.

CASADO, M. et al. Ethane: taking control of the enterprise. In: Proceedings of the 2007 conference on Applications, technologies, architectures, and protocols for computer communications. New York, NY, USA: ACM, 2007. (SIGCOMM '07), p. 1-12. ISBN 978-1-59593-713-1. Disponível em: <http://doi.acm.org/10.1145/1282380.1282382>. 
CATLETT, C.; TOOLE, J. Testbeds: Bridges from research to infrastructure. In: The Grid: Blueprint for a New Computing Infrastructure. [S.I.]: Morgan Kaufmann, 1998, (The Elsevier Series in Grid Computing). ISBN 1558604758.

CERF, V.; KAHN, R. A protocol for packet network intercommunication. Communications, IEEE Transactions on, v. 22, n. 5, p. 637-648, 1974. ISSN 0090-6778.

CERF, V. G.; CAIN, E. The DoD internet architecture model. Computer Networks (1976), v. 7, n. 5, p. 307-318, out. 1983. ISSN 0376-5075. Disponível em: <http://www.sciencedirect.com/science/article/pii/0376507583900429>.

CHOUDHARY, A. In-depth analysis of IPv6 security posture. In: 5th International Conference on Collaborative Computing: Networking, Applications and Worksharing, 2009. CollaborateCom 2009. [S.I.: s.n.], 2009. p. 1-7.

CHU, Y.-h. et al. A case for end system multicast. IEEE Journal on Selected Areas in Communications, v. 20, n. 8, p. 1456 - 1471, out. 2002. ISSN 0733-8716.

CISCO. The Zettabyte Era - Trends and Analysis. Visual Networking Index (VNI). maio 2013. Disponível em: <http://www.cisco.com/en/US/solutions/collateral/ns341/ns525/ns537/ns705/ns827/VNI_Hyperconnectivity_WP.html>.

CLARK, D. et al. Towards the Future Internet Architecture. IETF, 1991. (Request for Comments, 1287). Published: RFC 1287 (Informational). Disponível em: <http://www.ietf.org/rfc/rfc1287.txt>.

COMMISSION, E. ICT - Future Internet Research \& Experimentation. out. 2012. Disponível em: <http://cordis.europa.eu/fp7/ict/fire/home_en.html>.

. ICT Challenge 1: Pervasive and Trusted Network and Service Infrastructures. maio 2012. Disponível em: <http://cordis.europa.eu/fp7/ict/programme/challenge1_en.html>.

. The network of the future - Projects. 2012.

Http://cordis.europa.eu/fp7/ict/future-networks/projects_en.html. Disponível em: <http://cordis.europa.eu/fp7/ict/future-networks/projects_en.html>.

. ICT - The network of the future. jun. 2013. Disponível em: <http://cordis.europa.eu/fp7/ict/future-networks/home_en.html>.

CORUJO, D. et al. Using an open-source IEEE 802.21 implementation for network-based localized mobility management. IEEE Communications Magazine, v. 49, n. 9, p. 114-123, 2011. ISSN 0163-6804.

CPQD. Internet do Futuro: $C P q D$ se conecta a duas novas redes experimentais definidas por software | CPqD. 2013.

Disponível em: <http://www.cpqd.com.br/midia-eventos/fatos/fatos-203-

/internet-do-futuro-cpqd-se-conecta-a-duas-novas-redes-experimentais-definidas-por-softw 
DARPA. NewArch Project: Future-Generation Internet Architecture. set. 2003. Disponível em: <http://www.isi.edu/newarch/>.

DAVIES, E.; KRISHNAN, S.; SAVOLA, P. IPV6 Transition/Co-existence Security Considerations. IETF, 2007. (Request for Comments, 4942). Published: RFC 4942 (Informational). Disponível em: <http://www.ietf.org/rfc/rfc4942.txt>.

DAY, J. Patterns in Network Architecture: A Return to Fundamentals (paperback): A Return to Fundamentals. 1. ed. [S.I.]: Prentice Hall, 2008. ISBN 0137063385.

DEERING, S. Host extensions for IP multicasting. IETF, 1989. (Request for Comments, 1112). Published: RFC 1112 (Standard) Updated by RFC 2236. Disponível em: <http://www.ietf.org/rfc/rfc1112.txt>.

DEERING, S.; HINDEN, R. Internet Protocol, Version 6 (IPV6) Specification. IETF, 1995. (Request for Comments, 1883). Published: RFC 1883 (Proposed Standard) Obsoleted by RFC 2460. Disponível em: <http://www.ietf.org/rfc/rfc1883.txt>.

. Internet Protocol, Version 6 (IPV6) Specification. IETF, 1998. (Request for Comments, 2460). Published: RFC 2460 (Draft Standard) Updated by RFCs 5095, 5722, 5871. Disponível em: <http://www.ietf.org/rfc/rfc2460.txt>.

DHAMDHERE, A. et al. Measuring the deployment of IPv6: topology, routing and performance. In: Proceedings of the 2012 ACM Conference on Internet Measurement Conference. New York, NY, USA: ACM, 2012. (IMC '12), p. 537-550. ISBN 978-1-4503-1705-4. Disponível em: $<$ http://doi.acm.org/10.1145/2398776.2398832>.

DIOT, C. et al. Deployment issues for the IP multicast service and architecture. IEEE Network, v. 14, n. 1, p. 78 -88, fev. 2000. ISSN 0890-8044.

DORIA, A. et al. Forwarding and Control Element Separation (ForCES) Protocol Specification. IETF, mar. 2010. RFC 5810 (Proposed Standard). (Request for Comments, 5810). Disponível em: <http://www.ietf.org/rfc/rfc5810.txt>.

EDER, M.; NAG, S. Service Management Architectures Issues and Review. IETF, jan. 2001. RFC 3052 (Informational). (Request for Comments, 3052). Disponível em: <http://www.ietf.org/rfc/rfc3052.txt>.

EGEVANG, K.; FRANCIS, P. The IP Network Address Translator (NAT). IETF, 1994. (Request for Comments, 1631). Published: RFC 1631 (Informational) Obsoleted by RFC 3022. Disponível em: <http://www.ietf.org/rfc/rfc1631.txt>.

ELLIOTT, C. GENI: opening up new classes of experiments in global networking [Internet predictions]. IEEE Internet Computing, v. 14, n. 1, p. 39-42, fev. 2010. ISSN 1089-7801. 
EUBANKS, M.; CHIMENTO, P.; WESTERLUND, M. IPv6 and UDP Checksums for Tunneled Packets. IETF, 2013. (Request for Comments, 6935). Published: RFC 6935 (Proposed Standard). Disponível em: $<$ http://www.ietf.org/rfc/rfc6935.txt>.

EURESCOM. Future Internet Assembly - European Future Internet Portal. 2012. Disponível em: <http://www.future-internet.eu/home/future-internet-assembly.html>.

FELDMANN, A. Internet clean-slate design: what and why? SIGCOMM Comput. Commun. Rev., v. 37, n. 3, p. 59-64, jul. 2007. ISSN 0146-4833. Disponível em: <http://doi.acm.org/10.1145/1273445.1273453>.

FEMMINELLA, M. et al. Implementation and performance analysis of advanced IT services based on open source JAIN SLEE. In: 2011 IEEE 36th Conference on Local Computer Networks (LCN). [S.I.: s.n.], 2011. p. 746-753.

FIBRE. FIBRE Project - Future Internet Testbeds Experimentation Between Brazil and Europe. 2013. Disponível em: <http://www.fibre-ict.eu/>.

New FIBRE-BR members announced. 2013. Disponível $\overline{\mathrm{em}}$ : $<\mathrm{http}: / / \mathrm{www}$.fibre-ict.eu/index.php/component/content/article/85-new-fibre-br-members $>$.

FOUNDATION, N. S. NSF Future Internet Architecture Project. 2010. Http://www.nets-fia.net/. Disponível em: <http://www.nets-fia.net/>.

FOUNDATION, O. N. OpenFlow 1.2. Open Networking Foundation, dez. 2011. Disponível em: <https://www.opennetworking.org/images/stories/downloads/specification/openflow-spec-v1.2.pdf>.

. OpenFlow Switch Specification. Version 1.3.0. abr. 2012. Disponível em: $\overline{<\mathrm{http}}$ ://www.opennetworking.org/images/stories/downloads/specificationlopenflow-spec-v1.3.0.pdf>.

FULLER, V.; LI, T. Classless Inter-domain Routing (CIDR): The Internet Address Assignment and Aggregation Plan. IETF, ago. 2006. RFC 4632 (Best Current Practice). (Request for Comments, 4632). Disponível em: $<$ http://www.ietf.org/rfc/rfc4632.txt>.

GENI. GENI - Global Environment for Network Innovations. 2013. Disponível em: <http://www.geni.net/>.

GHODSI, A. et al. Intelligent design enables architectural evolution. In: Proceedings of the 10th ACM Workshop on Hot Topics in Networks. New York, NY, USA: ACM, 2011. (HotNets-X), p. 3:1-3:6. ISBN 978-1-4503-1059-8. Disponível em: <http://doi.acm.org/10.1145/2070562.2070565>.

GONT, F. Processing of IPv6 Atomic Fragments. IETF, 2013. (Request for Comments, 6946). Published: RFC 6935 (Proposed Standard). Disponível em: <http://www.ietf.org/rfc/rfc6935.txt>. 
GOTH, G. Software-Defined networking could shake up more than packets. IEEE Internet Computing, v. 15, n. 4, p. 6-9, ago. 2011. ISSN 1089-7801.

GREENBERG, A. et al. A clean slate 4D approach to network control and management. SIGCOMM Comput. Commun. Rev., v. 35, n. 5, p. 41-54, out. 2005. ISSN 0146-4833. Disponível em: <http://doi.acm.org/10.1145/1096536$.1096541>$.

GREENE, K. TR10: Software-Defined networking. MIT Technology Review, v. 112, n. 2, abr. 2009. Disponível em: <http://www.technologyreview.com/web/22120/>.

GROUP, I. E. S.; HINDEN, R. Applicability Statement for the Implementation of Classless Inter-Domain Routing (CIDR). IETF, 1993. (Request for Comments, 1517). Published: RFC 1517 (Historic). Disponível em: $<$ http://www.ietf.org/rfc/rfc1517.txt>.

Applicability Statement for the Implementation of Classless Inter-Domain Routing (CIDR). IETF, set. 1993. RFC 1517 (Historic). (Request for Comments, 1517). Disponível em: <http://www.ietf.org/rfc/rfc1517.txt>.

GUIMARãES, C. et al. Empowering software defined wireless networks through media independent handover management. In: Globecom 2013 Next Generation Networking Symposium (GC13 NGN). Atlanta, USA: [s.n.], 2013.

GURBANI, V. K.; HAERENS, F.; RASTOGI, V. Interworking SIP and Intelligent Network (IN) Applications. IETF, jan. 2005. RFC 3976 (Informational). (Request for Comments, 3976). Disponível em: <http://www.ietf.org/rfc/rfc3976.txt>.

HALPERN, J.; SALIM, J. H. Forwarding and Control Element Separation (ForCES) Forwarding Element Model. IETF, mar. 2010. RFC 5812 (Proposed Standard). (Request for Comments, 5812). Disponível em: $<$ http://www.ietf.org/rfc/rfc5812.txt>.

HAN, D. et al. XIA: efficient support for evolvable internetworking. In: Proceedings of the 9th USENIX conference on Networked Systems Design and Implementation. Berkeley, CA, USA: USENIX Association, 2012. (NSDI'12), p. 23-23. Disponível em: <http://dl.acm.org/citation.cfm? $\mathrm{id}=2228298.2228330>$.

HANDLEY, M. Why the internet only just works. BT Technology Journal, v. 24, n. 3, p. 119-129, jul. 2006. ISSN 1358-3948. Disponível em: $<$ http://dx.doi.org/10.1007/s10550-006-0084-z>.

HANNA, S.; PATEL, B.; SHAH, M. Multicast Address Dynamic Client Allocation Protocol (MADCAP). IETF, dez. 1999. RFC 2730 (Proposed Standard). (Request for Comments, 2730). Disponível em: <http://www.ietf.org/rfc/rfc2730.txt>. 
HAZEYAMA, $\mathrm{H}$. et al. How much can we survive on an IPv6 network?: experience on the IPv6 only connectivity with NAT64/DNS64 at WIDE camp 2011 autumn. In: Proceedings of the 7th Asian Internet Engineering Conference. New York, NY, USA: ACM, 2011. (AINTEC '11), p. 144-151. ISBN 978-1-4503-1062-8. Disponível em: <http://doi.acm.org/10.1145/2089016.2089041>.

HINDEN, R.; DEERING, S. IP Version 6 Addressing Architecture. IETF, 1995. (Request for Comments, 1884). Published: RFC 1884 (Historic) Obsoleted by RFC 2373. Disponível em: <http://www.ietf.org/rfc/rfc1884.txt>.

. IP Version 6 Addressing Architecture. IETF, 1998. (Request for Comments, 2373). Published: RFC 2373 (Proposed Standard) Obsoleted by RFC 3513. Disponível em: <http://www.ietf.org/rfc/rfc2373.txt>.

. IPv6 Multicast Address Assignments. IETF, 1998. (Request for Comments, 2375). Published: RFC 2375 (Informational). Disponível em: $<$ http://www. ietf.org/rfc/rfc2375.txt>.

. Internet Protocol Version 6 (IPv6) Addressing Architecture. IETF, 2003. (Request for Comments, 3513). Published: RFC 3513 (Proposed Standard) Obsoleted by RFC 4291. Disponível em: <http://www.ietf.org/rfc/rfc3513.txt>.

. IP Version 6 Addressing Architecture. IETF, 2006. (Request for Comments, 4291). Published: RFC 4291 (Draft Standard) Updated by RFCs 5952, 6052. Disponível em: <http://www.ietf.org/rfc/rfc4291.txt>.

HOLDREGE, M.; SRISURESH, P. Protocol Complications with the IP Network Address Translator. IETF, 2001. (Request for Comments, 3027). Published: RFC 3027 (Informational). Disponível em: <http://www.ietf.org/rfc/rfc3027.txt>.

HOSSEINI, M. et al. A survey of application-layer multicast protocols. Commun. Surveys Tuts., v. 9, n. 3, p. 58-74, jul. 2007. ISSN 1553-877X. Disponível em: <http://dx.doi.org/10.1109/COMST.2007.4317616>.

HUNG, T.; DUONG, V. Mobile IPv6 fast handover techniques. In: 2011 13th International Conference on Advanced Communication Technology (ICACT). [S.I.: s.n.], 2011. p. 1304-1308.

IDC. Worldwide Smart Connected Device Market Crossed One Billion Shipments in 2012. mar. 2013. Disponível em: <http://www.idc.com/getdoc.jsp?containerld=prUS24037713 $>$.

IEEE. IEEE standard for local and metropolitan area networks - part 21: Media independent handover. IEEE Std 802.21-2008, p. c1 -301, 2009.

INFOBLOX. FlowForwarding Documentation. jun. 2012. Http://flowforwarding.org/resources/doc. Disponível em: <http://flowforwarding.org/resources/doc>. Acesso em: 25 jun. 2012. 
IPT. Projeto MyFire premia projetos inovadores em redes. set. 2011. Disponível em: <http://www.ipt.br/noticias_interna.php?id_noticia=405>.

IPv6Now. IPv6 RFCs. maio 2013. Disponível em: <http://www.ipv6now.com.au/RFC.php>.

JACOBSON, V. et al. Networking named content. Commun. ACM, v. 55 , n. 1, p. 117-124, jan. 2012. ISSN 0001-0782. Disponível em: $<$ http://doi.acm.org/10.1145/2063176.2063204>.

KAWAMURA, S.; KAWASHIMA, M. A Recommendation for IPv6 Address Text Representation. IETF, 2010. (Request for Comments, 5952). Published: RFC 5952 (Proposed Standard). Disponível em: <http://www.ietf.org/rfc/rfc5952.txt>.

KERNER, S. OpenFlow Protocol 1.3.0 Approved. maio 2012. Disponível em: $<$ http://www.enterprisenetworkingplanet.com/nethub/openflow-protocol-1.3.0-approved.html>.

KHOSRAVI, H.; ANDERSON, T. Requirements for Separation of IP Control and Forwarding. IETF, nov. 2003. RFC 3654 (Informational). (Request for Comments, 3654). Disponível em: <http://www.ietf.org/rfc/rfc3654.txt>.

KOHLER, E. et al. The click modular router. ACM Trans. Comput. Syst., v. 18 , n. 3, p. 263-297, ago. 2000. ISSN 0734-2071. Disponível em: <http://doi.acm.org/10.1145/354871.354874>.

KOODLI, R. Mobile IPv6 Fast Handovers. IETF, 2009. (Request for Comments, 5568). Published: RFC 5568 (Proposed Standard). Disponível em: <http://www.ietf.org/rfc/rfc5568.txt>.

KOPONEN, T. et al. Onix: a distributed control platform for large-scale production networks. In: Proceedings of the 9th USENIX conference on Operating systems design and implementation. Berkeley, CA, USA: USENIX Association, 2010. (OSDl'10), p. 1-6. Disponível em: <http://dl.acm.org/citation.cfm?id=1924943.1924968>.

KRISHNAN, S. Handling of Overlapping IPv6 Fragments. IETF, 2009. (Request for Comments, 5722). Published: RFC 5722 (Proposed Standard) Updated by RFC 6946. Disponível em: <http://www.ietf.org/rfc/rfc5722.txt>.

KRISHNAN, S. et al. A Uniform Format for IPv6 Extension Headers. IETF, 2012. (Request for Comments, 6564). Published: RFC 6564 (Proposed Standard). Disponivel em: <http://www.ietf.org/rfc/rfc6564.txt>.

LANTZ, B.; HELLER, B.; McKeown, N. A network in a laptop: rapid prototyping for software-defined networks. Proceedings of the Ninth ACM SIGCOMM Workshop on Hot Topics in Networks, p. 19:1-19:6, 2010. ACM ID: 1868466. 
LAO, L. et al. A comparative study of multicast protocols: top, bottom, or in the middle? In: Proceedings IEEE INFOCOM 2005. 24th Annual Joint Conference of the IEEE Computer and Communications Societies. [S.I.: s.n.], 2005. v. 4, p. $2809-2814$ vol. 4.

MARTINEZ, A. et al. Toward a new addressing scheme for a service-centric internet. In: 2012 IEEE International Conference on Communications (ICC). [S.I.: s.n.], 2012. p. $6463-6467$.

MCKEOWN, N. et al. OpenFlow: enabling innovation in campus networks. SIGCOMM Comput. Commun. Rev., v. 38, n. 2, p. 69-74, mar. 2008. ISSN 0146-4833. ACM ID: 1355746.

MEHAR. MEHAR. Mondial Entities Horizontally Addressed by Requirements. 2011. Disponível em: <www.mehar.facom.ufu.br>.

MOBICENTS. Mobicents JAIN SLEE. 2012.

Http://www.mobicents.org/slee/intro.html. Disponível em: <http://www.mobicents.org/slee/intro.html>.

MyFire. All About MyFire. fev. 2011. Disponível em: <http://www.my-fire.eulabout>.

NAOUS, J. et al. Implementing an OpenFlow switch on the NetFPGA platform. In: Proceedings of the 4th ACM/IEEE Symposium on Architectures for Networking and Communications Systems. San Jose, California: ACM, 2008. (ANCS '08), p. 1-9. ISBN 978-1-60558-346-4. ACM ID: 1477944.

NCC, R. IPv6 Enabled Networks. jul. 2013. Disponível em: <http://v6asns.ripe.net/v/6?s=_ALL>.

NELSON, S. C.; BHANAGE, G.; RAYCHAUDHURI, D. GSTAR: generalized Storage-Aware routing for mobilityfirst in the future mobile internet. In: Proceedings of the sixth international workshop on MobiArch. New York, NY, USA: ACM, 2011. (MobiArch '11), p. 19-24. ISBN 978-1-4503-0740-6. Disponível em: <http://doi.acm.org/10.1145/1999916.1999922>.

NSF. FIND - Future Internet Design. 2009. Disponível em: <http://www.nets-find.net/>.

OFELIA. OpenFlow in Europe - Linking Infrastructure and Applications. 2011. Disponivel em: <http://www.fp7-ofelia.eu/about-ofelia/>. Acesso em: 07 jul. 2011.

\section{OFELIA Second Open Call for OpenFlow Ex-}

periments Success and New Partners. jul. 2012.

Http://www.fp7-ofelia.eu/news-and-events/press-releases/ofelia-2nd-open-call-success/. Disponível em: <http://www.fp7-ofelia.eu/news-and-events/press-releases/ofelia-2nd-open-call-success/>. 
. OFELIA Second Open Call for OpenFlow Experiments Success and New Partners. jul. 2012. Disponível em: <http://www.fp7-ofelia.eu/news-and-events/press-releases/ofelia-2nd-open-call-success/>.

. OFELIA Second Open Call for OpenFlow Experiments Success and New Partners. jul. 2012. Disponível em: <http://www.fp7-ofelia.eu/news-and-events/press-releases/ofelia-2nd-open-call-success/>.

PEREIRA, J. de S.; KOFUJI, S.; ROSA, P. Horizontal address ontology in internet architecture. In: New Technologies, Mobility and Security (NTMS), 2009 3rd International Conference on. [S.I.: s.n.], 2009. p. 1 -6.

PEREIRA, J. H. d. S. et al. Title model ontology for future internet networks. In: Future Internet Assembly 2011: Achievements and Technological Promises. Future Internet: Achievements and Promising Technology: Springer-Verlag, 2011, (LNCS, v. 6656). p. 465. ISBN 978-3-642-20897-3.

PEREIRA, J. H. de S.; KOFUJI, S. T.; ROSA, P. F. Horizontal addressing by title in a next generation internet. In: 2010 Sixth International Conference on Networking and Services (ICNS). [S.I.]: IEEE, 2010. p. 7-11. ISBN 978-1-4244-5927-8.

PERKINS, C. IP Mobility Support. IETF, 1996. (Request for Comments, 2002). Published: RFC 2002 (Proposed Standard) Obsoleted by RFC 3220, updated by RFC 2290. Disponível em: <http://www.ietf.org/rfc/rfc2002.txt>.

. IP Mobility Support for IPv4. IETF, 2002. (Request for Comments, 3344). Published: RFC 3344 (Proposed Standard) Obsoleted by RFC 5944, updated by RFCs 4636, 4721. Disponível em: <http://www.ietf.org/rfc/rfc3344.txt>.

PERKINS, C.; JOHNSON, D.; ARKKO, J. Mobility Support in IPv6. IETF, 2011. (Request for Comments, 6275). Published: RFC 6275 (Proposed Standard). Disponível em: <http://www.ietf.org/rff/rfc6275.txt>.

PERKINS, C. E.; JOHNSON, D. B. Mobility support in IPv6. In: Proceedings of the 2nd annual international conference on Mobile computing and networking. New York, NY, USA: ACM, 1996. (MobiCom '96), p. 27-37. ISBN 0-89791-872-X. Disponível em: <http://doi.acm.org/10.1145/236387.236400>.

PFAFF, B. et al. OpenFlow Switch Specification. Version 1.1.0 Implemented. Open Networking Foundation, fev. 2011. Disponível em: <http://www.openflow.org/documents/openflow-spec-v1.1.0.pdf >. Acesso em: 07 jul. 2011.

POSTEL, J. Assigned numbers. IETF, 1980. (Request for Comments, 762). Published: RFC 762 (Historic) Obsoleted by RFC 770. Disponível em: $<$ http://www.ietf.org/rfc/rfc762.txt>.

. DoD standard Internet Protocol. IETF, 1980. (Request for Comments, 760). Published: RFC 760 Obsoleted by RFC 791, updated by RFC 777. Disponível em: <http://www.ietf.org/rfc/rfc760.txt>. 
. Internet Protocol. IETF, 1981. (Request for Comments, 791).

Published: RFC 791 (Standard) Updated by RFC 1349. Disponível em: $<$ http://www.ietf.org/rfc/rfc791.txt>.

. Internet Protocol. IETF, set. 1981. RFC 791 (Standard). (Request for Comments, 791). Updated by RFC 1349. Disponível em: <http://www.ietf.org/rfc/rfc791.txt>.

RAFIEE, H.; LOWIS, M. von; MEINEL, C. IPv6 deployment and spam challenges. IEEE Internet Computing, v. 16, n. 6, p. 22-29, 2012. ISSN 1089-7801.

RAHMAN, M. et al. Performance comparison between MIPV6 and SEMO6. In: 2010 IEEE Global Telecommunications Conference (GLOBECOM 2010). [S.I.: s.n.], 2010. p. 1-5.

REKHTER, Y.; LI, T. A Border Gateway Protocol 4 (BGP-4). mar. 1995. Http://tools.ietf.org/html/rfc1771. Disponível em: <http://tools.ietf.org/html/rfc1771>.

REXFORD, J.; DOVROLIS, C. Future internet architecture: clean-slate versus evolutionary research. Communications of the ACM, v. 53, n. 9, p. 36-40, 2010. ISSN 0001-0782.

RNP. Internet do Futuro. set. 2011. Disponível em: <https://www.rnp.br/pd/if/>.

ROBERTS, J. The clean-slate approach to future internet design: a survey of research initiatives. annals of telecommunications - annales des télécommunications, v. 64, n. 5-6, p. 271-276, maio 2009. ISSN 0003-4347. Disponível em: <http://www.springerlink.com/content/e240776641607136/>. Acesso em: 07 jul. 2011.

ROMDHANI, I. et al. IP mobile multicast: Challenges and solutions. IEEE Communications Surveys Tutorials, v. 6, n. 1, p. 18-41, 2004. ISSN 1553-877X.

SAHA, D. et al. Mobility support in IP: a survey of related protocols. IEEE Network, v. 18, n. 6, p. 34-40, 2004. ISSN 0890-8044.

SALTZER, J. On the Naming and Binding of Network Destinations. IETF, 1993. (Request for Comments, 1498). Published: RFC 1498 (Informational). Disponível em: <http://www.ietf.org/rfc/rfc1498.txt>.

SAVOLA, P. Overview of the Internet Multicast Addressing Architecture. IETF, 2011. (Request for Comments, 6308). Published: RFC 6308 (Informational). Disponível em: <http://www.ietf.org/rfc/rfc6308.txt>.

SCHMIDT, D. et al. Pattern-Oriented Software Architecture Volume 2:

Patterns for Concurrent and Networked Objects. Volume 2. [S.I.]: Wiley, 2000. ISBN 0471606952. 
SCHMIDT, T.; WAEHLISCH, M.; FAIRHURST, G. Multicast Mobility in Mobile IP Version 6 (MIPv6): Problem Statement and Brief Survey. IETF, 2010. (Request for Comments, 5757). Published: RFC 5757 (Informational). Disponível em: <http://www.ietf.org/rfc/rfc5757.txt>.

SCHULZRINNE, H. et al. RTP: A Transport Protocol for Real-Time Applications. IETF, 2003. (Request for Comments, 3550). Published: RFC 3550 (Standard) Updated by RFCs 5506, 5761, 6051. Disponível em: $<$ http://www.ietf.org/rfc/rfc3550.txt>.

SESKAR, I. et al. MobilityFirst future internet architecture project. In: Proceedings of the 7th Asian Internet Engineering Conference. New York, NY, USA: ACM, 2011. (AINTEC '11), p. 1-3. ISBN 978-1-4503-1062-8. Disponível em: <http://doi.acm.org/10.1145/2089016.2089017>.

SHANNON, C. E.; WEAVER, W. The mathematical theory of communication. Urbana: University of Illinois Press, 1949. ISBN 9780252725463.

SILVA, F. d. O. et al. Enabling network mobility by using IEEE 802.21 integrated with the entity title architecture. In: Anais do IV Workshop de Pesquisa Experimental na Internet do Futuro (WPEIF). Brasília: Sociedade Brasileira de Computação, 2013. p. 29-34. ISBN 2177-496X.

. Implementing the domain title service atop OpenFlow. In: Anais do III Workshop de Pesquisa Experimental na Internet do Futuro (WPEIF). Porto Alegre: Sociedade Brasileira de Computação, 2012. p. 38-41.

Domain title service for future internet networks. In: Anais do II Workshop de Pesquisa Experimental na Internet do Futuro (WPEIF). Campo Grande: SBC, 2011. ISBN 2177-496X.

SILVA, F. de O. et al. Semantically enriched services to understand the need of entities. In: ÁLVAREZ, F. et al. (Ed.). The Future Internet. Springer Berlin / Heidelberg, 2012, (Lecture Notes in Computer Science, v. 7281). p. 142-153. ISBN 978-3-642-30240-4. Disponível em: <http://www.springerlink.com/content/1222874ul734676k/abstract/>.

. On the analysis of multicast traffic over the entity title architecture. In: 2012 18th IEEE International Conference on Networks (ICON). [S.I.: s.n.], 2012. p. 30-35.

. Enabling future internet architecture research and experimentation by using software defined networking. In: 2012 European Workshop on Software Defined Networking (EWSDN). [S.I.: s.n.], 2012. p. 73-78.

SNOEREN, A.; BALAKRISHNAN, H.; KAASHOEK, M. Reconsidering internet mobility. In: Proceedings of the Eighth Workshop on Hot Topics in Operating Systems, 2001. [S.I.: s.n.], 2001. p. 41-46. 
SOLIMAN, H. Mobile IPv6 Support for Dual Stack Hosts and Routers. IETF, 2009. (Request for Comments, 5555). Published: RFC 5555 (Proposed Standard). Disponivel em: <http://www.ietf.org/rfc/rfc5555.txt>.

SOLIMAN, H. et al. Hierarchical Mobile IPv6 (HMIPv6) Mobility Management. IETF, 2008. (Request for Comments, 5380). Published: RFC 5380 (Proposed Standard). Disponível em: <http://www.ietf.org/rfc/rfc5380.txt>.

SRISURESH, P.; EGEVANG, K. Traditional IP Network Address Translator (Traditional NAT). IETF, 2001. (Request for Comments, 3022). Published: RFC 3022 (Informational). Disponível em: <http://www.ietf.org/rfc/rfc3022.txt>.

STANFORD. Clean Slate Design for the Internet. 2006. Disponível em: $<$ http://cleanslate.stanford.edu/index.php $>$.

STEENKISTE, P. Implementing XIA forwarding on Openflow. fev. 2011. http://www.cs.cmu.edu/ prs/REU-XIA-web.html. Disponível em: <http://www.cs.cmu.edu/pprs/REU-XIA-web.html>.

TAIB, A. H. M.; ALI, W. N. A. W.; SHAARI, N. S. ICMPV6 vulnerability: The importance of threat model and SF-ICMP6. International Journal of Mobile Computing and Multimedia Communications, v. 5, n. 2, p. 78-100, 2013. ISSN 1937-9412, 1937-9404. Disponível em: <http://www.igi-global.com/article/icmpv6-vulnerability-importance-threat-model/78387> .

TANENBAUM, A. S. Computer Networks. 4. ed. [S.I.]: Prentice Hall, 2002. ISBN 0130661023.

TELECOMUNICAçõES, C. de Pesquisa e Desenvolvimento em. OpenFlow 1.2 Tutorial. jul. 2012. https://github.com/CPqD/OpenFlow-1.2-Tutorial. Disponivel em: <https://github.com/CPqD/OpenFlow-1.2-Tutorial>.

THALER, D.; HANDLEY, M.; ESTRIN, D. The Internet Multicast Address Allocation Architecture. IETF, 2000. (Request for Comments, 2908). Published: RFC 2908 (Historic) Obsoleted by RFC 6308. Disponível em: $<$ http://www.ietf.org/rfc/rfc2908.txt>.

TRIPP, S. D.; BICHELMEYER, B. Rapid prototyping: An alternative instructional design strategy. Educational Technology Research and Development, v. 38, n. 1, p. 31-44, jan. 1990. ISSN 1042-1629, 1556-6501. Disponível em: <http://link.springer.com/article/10.1007/BF02298246>.

TSELENTIS, G. et al. Towards the future internet a European research perspective. Amsterdam Netherlands ;;Washington DC: IOS Press, 2009. ISBN 9781607504313.

UNION, I. T. Security architecture for Open Systems Interconnection for CCITT applications. Recommendation X.800. International Telecommunication Union, 1991. (Series X: Data Networks and Open System Communication). Disponível em: <http://www.itu.int/rec/T-REC-X.800/en>. 
. Information tecnology - Open Systems Interconnection - Basic Reference Model: The Basic Model. ITU-T Recommendation X.200. International Telecommunication Union, 1994. (Series X: Data Networks and Open System Communication). Disponível em: <http://www.itu.int/rec/T-REC-X.200-199407-I/en>.

. Information tecnology - Open Systems Interconnection - Basic Reference Model: Naming and addresing. ITU-T Recommendation X.650. International Telecommunication Union, 1996. (Series X: Data networks, open system communications and security). Disponível em: $<$ http://www.itu.int/rec/T-REC-X.650-199610-I/en>.

. General series Intelligent Network Recommendation structure. International Telecommunication Union, 1997. (SERIES Q: SWITCHING AND SIGNALLING - Intelligent Network). Disponível em: <http://www.itu.int/rec/T-REC-Q.1200-199709-I/en>.

WOOD, G. IPv6: making room for the world on the future internet. IEEE Internet Computing, v. 15, n. 4, p. 88-89, 2011. ISSN 1089-7801.

YANG, L. et al. Forwarding and Control Element Separation (ForCES) Framework. IETF, abr. 2004. RFC 3746 (Informational). (Request for Comments, 3746). Disponível em: <http://www.ietf.org/rfc/rfc3746.txt>.

YIU, W.-P. K.; CHAN, S.-H. G. Offering data confidentiality for multimedia overlay multicast: Design and analysis. ACM Trans. Multimedia Comput. Commun. Appl., v. 5, n. 2, p. 13:1-13:23, nov. 2008. ISSN 1551-6857. Disponível em: <http://doi.acm.org/10.1145/1413862.1413866>.

ZAHARIADIS, T. et al. Towards a future internet architecture. In: DOMINGUE, J. et al. (Ed.). The Future Internet. Future Internet Assembly 2011: Achievements and Technological Promises. Berlin, Heidelberg: Springer-Verlag, 2011, (LNCS, v. 6656). p. 7-18. ISBN 978-3-642-20897-3. Disponível em: $<$ http://link.springer.com/chapter/10.1007/978-3-642-20898-0_1>. 


\section{APÊNDICE A - DEFINIÇÃO DE TIPOS DE DADOS}

Este apêndice descreve os tipos de dados utilizados pelos protocolos ETCP e DSTCP. A tabela 11 descreve tipos de dados utilizados no capítulo C indicando a partir de quais tipos básicos os mesmos são derivados.

Tabela 11: Tipos de Dados Utilizados nos Protocolos ETCP e DTSCP.

\begin{tabular}{|c|c|c|}
\hline TIPO DO DADO & DERIVADO DE & OBSERVAÇÕES \\
\hline PRIMITIVE-TYPE & ENUMERATED & $\begin{array}{l}\text { O tipo da primitiva que pode assumir os } \\
\text { seguinte valores: } \\
\text { ETCP-ENTITY-REGISTER = } 0 ; \\
\text { ETCP-ENTITY-UNREGISTER = } ; \text {; } \\
\text { ETCP-WORKSPACE-CREATE = } ; \\
\text { ETCP-WORKSPACE-ATTACH = } ; \\
\text { ETCP-WORKSPACE-DETACH = } 4 ; \\
\text { ETCP-WORKSPACE-DELETE = } ; \\
\text { ETCP-WORKSPACE-MODIFY = } 6 ; \\
\text { DTSCP-WORKSPACE-LOOKUP = } 7 ; \\
\text { DTSCP-WORKSPACE-ADVERTISE = } 8 ; \\
\text { DTSCP-DTSA-REGISTER = } 9 \\
\text { DTSCP-DSTA-MESSAGE = } 10 ;\end{array}$ \\
\hline PRIMITIVE-ID-TYPE & UNSIGNED-INT(1) & $\begin{array}{l}\text { Número inteiro utilizado para identificar } \\
\text { um par requisição/reposta }\end{array}$ \\
\hline RETURN-STATUS-TYPE & ENUMERATED & $\begin{array}{l}\text { Condição de retorno associada a uma } \\
\text { requisição: } \\
\text { SUCCESSO = } 0 \text { e FALHA = } 1\end{array}$ \\
\hline REQUIREMENT-TYPE & $\begin{array}{l}\text { SEQUENCE[ } \\
\text { UNSIGNED-INT(1), } \\
\text { UNSIGNED-INT(2), } \\
\text { OCTET-STRING] }\end{array}$ & $\begin{array}{l}\text { Baseado em um abordagem TLV (Type, Length } \\
\text { and Value) consiste de um tipo genérico com } \\
\text { três campos a fim de descrever um requisito ou } \\
\text { capacidade de uma entidade relacionada com a } \\
\text { sua comunicação. O primeiro campo } \\
\text { representa um tipo único; o segundo campo é o } \\
\text { comprimento associado com o valor e } \\
\text { finalmente, o valor que está contido neste } \\
\text { campo genérico }\end{array}$ \\
\hline
\end{tabular}


Tabela 11: (Cont.)Tipos de Dados Utilizados nos Protocolos ETCP e DTSCP.

\begin{tabular}{lll}
\hline TIPO DO DADO & DERIVADO DE & OBSERVAÇÕES \\
\hline \hline TITLE-TYPE & OCTET-STRING & $\begin{array}{l}\text { Um vetor de comprimento arbitrário de } \\
\text { octetos, ou seja, oito bits. }\end{array}$ \\
\hline DTSA-PATH-TYPE & $\begin{array}{l}\text { SEQUENCE[ } \\
\text { TITLE-TYPE, } \\
\text { OCTET-STRING] }\end{array}$ & $\begin{array}{l}\text { Par que contém o Título de um DTSA e o custo } \\
\text { associado aquele DTSA }\end{array}$ \\
\hline
\end{tabular}




\section{APÊNDICE B - RESULTADOS}

Este apêndice descreve os frutos deste trabalho de pesquisa que podem ser caracterizados como publicações, prêmios, o estabelecimento de colaborações nacionais e internacionais e obtenção de fomento para o desenvolvimento do trabalho.

A Tabela 12 sumariza, em ordem cronológica, a produção bibliográfica resultante deste trabalho.

Tabela 12: Produção Bibliográfica Resultante Deste Trabalho.

\begin{tabular}{lll}
\hline \hline \multicolumn{1}{c}{ Título } & Ano & Tipo da Produção \\
\hline \hline $\begin{array}{l}\text { Domain Title Service for Future Internet Networks } \\
\text { (SILVA et al., 2011) }\end{array}$ & 2011 & Anais de Congresso \\
\hline $\begin{array}{l}\text { Semantically enriched services to understand the } \\
\text { need of entities (SILVA et al., 2012a) }\end{array}$ & 2012 & Capítulo de Livro \\
\hline $\begin{array}{l}\text { Implementing the Domain Title Service atop Open- } \\
\text { Flow (SILVA et al., 2012) }\end{array}$ & & Anais de Congresso \\
\hline $\begin{array}{l}\text { Enabling Future Internet Architecture Research and } \\
\text { Experimentation by Using Software Defined Networ- } \\
\text { king (SILVA et al., 2012c) }\end{array}$ & Anais de Congresso \\
\hline $\begin{array}{l}\text { On the analysis of multicast traffic over the Entity } \\
\text { Title Architecture (SILVA et al., 2012b) }\end{array}$ & 2012 & Anais de Congresso \\
\hline $\begin{array}{l}\text { Enabling Network Mobility by Using IEEE 802.21 In- } \\
\text { tegrated with the Entity Title Architecture (SILVA et } \\
\text { al., 2013) }\end{array}$ & Anais de Congresso \\
\hline $\begin{array}{l}\text { Empowering Software Defined Wireless Networks } \\
\text { Through Media Independent Handover Manage- } \\
\text { ment (GUIMARãES et al., 2013) }\end{array}$ & Anais de Congresso \\
\hline
\end{tabular}

Em 2011, o projeto MyFIRE (MyFire, 2011), com financiamento FP7, e que possuía como um de seus objetivos o fomento no uso de instalações 
experimentais (testbeds) relacionadas com a pesquisa para a Internet do futuro, propôs uma competição a fim de escolher propostas para experimentação em testbeds europeias. A proposta "Experimenting Domain Title Service to Meet Mobility and Multicast Aggregation by using OpenFlow" foi escolhida (IPT, 2011) e além da possibilidade de utilização da testbed do projeto OFELIA, foi agraciada com o custeio de uma viagem para a Future Internet Week realizada de 24 a 28 de outubro de 2011 em Poznan, na Polônia.

Em 2012, uma proposta intitulada "Mobilidade na Internet do Futuro: Integrando o Domain Title Service (DTS) ao protocolo IEEE 802.21" foi submetida ao Programa Santander de Bolsas de Mobilidade Internacional foi aceita. A proposta envolvia a integração deste trabalho de pesquisa com a pesquisa conduzida pelo Prof. Dr. Rui Aguiar, da Universidade de Aveiro. O fomento permitiu uma visita técnica a Portugal, permitiu a realização de trabalhos conjuntos e ainda contribui para o estreitamento de laços entre a Universidade de Aveiro, a Universidade de São Paulo e a Universidade Federal de Uberlândia. Além disso esta colaboração resultou em um termo de cooperação assinado entre a Universidade de Aveiro e a Universidade Federal de Uberlândia.

Ainda em 2012, o projeto OFELIA (OFELIA, 2011) publicou uma chamada aberta à comunidade científica (OFELIA, 2012a) visando agregar novos parceiros ao projeto. A proposta chamada EDOBRA (ATNOG, 2012), fruto deste trabalho de pesquisa foi aceita (OFELIA, 2012c). Esta proposta, elaborada conjunto com a Escola Politécnica da Universidade de São Paulo, o Instituto de Telecomunicações, da Universidade de Aveiro em Portugal e a Faculdade de Computação da Universidade Federal de Uberlândia, é fruto da colaboração que foi iniciada ainda no ano de 2011, durante a viagem à Poznan. O fomento permitiu ampliar o trabalho envolvendo a ETArch e além disso permitiu ampliar a extensão física do OFELIA, através de uma implantação de uma 
ilha em Uberlândia(MG) que está interligada às outras ilhas (OFELIA, 2012b)

O trabalho permitiu a construção de um diálogo com a comunidade científica nacional no tema relacionado à pesquisa experimental em Internet do futuro e em 2013, a Universidade Federal de Uberlândia foi convidada a associar-se (FIBRE, 2013b) ao projeto FIBRE que conta com financiamento conjunto Brasil (CNPq) e Comissão Europeia (FP7). Como resultado, na Faculdade de Computação da Universidade Federal de Uberlândia, no mesmo espaço onde está hospedada a ilha do OFELIA, está em implantação uma ilha do FIBRE. Esta ilha estará interligada tanto com a Europa como com diversas outras instituições no Brasil, ampliando assim as possibilidades de futuras colaborações e pesquisas conjuntas envolvendo a próxima Geração de Redes de Computadores. 


\section{APÊNDICE C - PROTOCOLOS DA ARQUITETURA ETARCH}

O objetivo deste apêndice é detalhar as primitivas do protocolo ETCP (Entity Title Protocol) utilizado pelas entidades para comunicação com o DTS bem como as primitivas do protocolo DTSCP (Domain Title Service Control Protocol) utilizado para a comunicação entre os DTSAs.

Inicialmente é apresentado o formato da PDU (Protocol Data Unit) (UNION, 1994) utilizada e na sequência, os serviços de cada protocolo e as primitivas envolvidas.

\section{C.1 Formato das Primitivas}

Ambos os protocolos (ETCP e DTSCP) utilizam um formato padrão de primitiva. As requisições (request) são apresentadas na Figura 33(a). Da mesma forma, a Figura 33(b) apresenta o formato padrão das respostas a uma dada requisição. A Tabela 13 apresenta a semântica dos campos da primitiva de controle utilizada na requisição (CONTROL-PRIMITIVE) e na resposta (CONTROL-RESPONSE). Os tipos de dados abstratos apresentados são definidos no apêndice $A$.

A PDU dos protocolos ainda contém o título do Workspace através do qual as primitivas são enviadas. Logo em seguida, existe um enchimento 


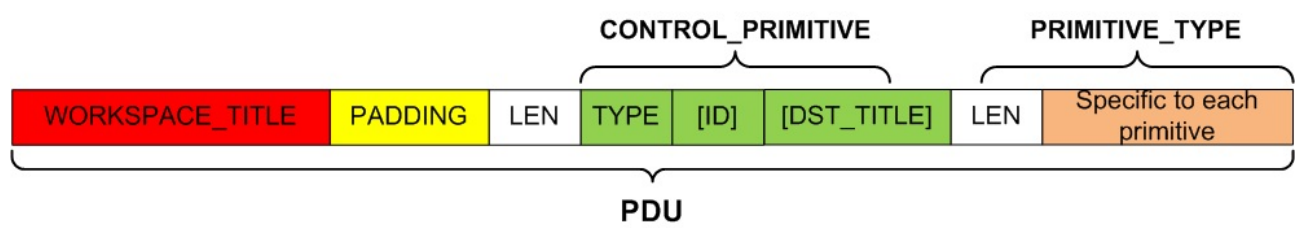

(a) Formato Geral da Requisição

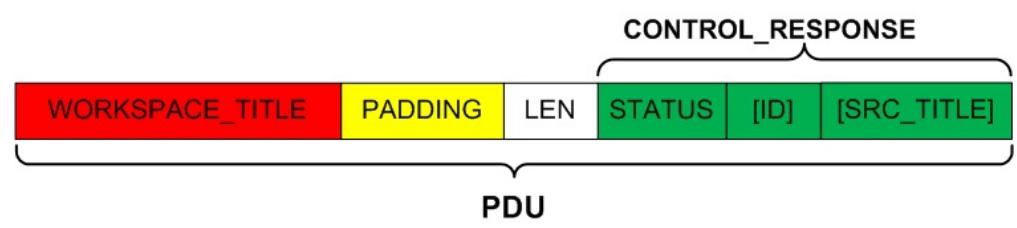

(b) Formato Geral da Resposta

Figura 33: Formato Geral da Primitiva dos Protocolos ETCP e DTSCP.

(PADDING) com dois bytes. Antes de cada primitiva é necessário incluir o seu comprimento em bytes, conforme indicado pelo campo $L E N$, nas Figuras 33(a) e 33(b).

Tabela 13: Semântica dos Campos das Primitivas de Controle

\begin{tabular}{|c|c|c|c|}
\hline NOME & TIPO DO DADO & PRESENÇA & DESCRIÇÃO \\
\hline TYPE & PRIMITIVE_TYPE & Necessário & Identifica o tipo da primitiva \\
\hline ID & PRIMITIVE_ID_TYPE & Opcional & $\begin{array}{l}\text { Identificador atribuído à requisi- } \\
\text { ção a fim de realizar uma associ- } \\
\text { ação com a resposta correspon- } \\
\text { dente }\end{array}$ \\
\hline DST-TITLE & TITLE-TYPE & Opcional & $\begin{array}{l}\text { Titulo da Entidade de destino. } \\
\text { Neste caso a primitiva será envi- } \\
\text { ada através de um dado Works- } \\
\text { pace porém somente a entidade } \\
\text { com o referido Titulo é que pro- } \\
\text { cessará a requisição }\end{array}$ \\
\hline STATUS & PRIMITIVE-ID-TYPE & Necessário & $\begin{array}{l}\text { Representa o status de uma re- } \\
\text { quisição que foi previamente en- } \\
\text { viada por uma entidade }\end{array}$ \\
\hline SRC-TITLE & TITLE-TYPE & Opcional & $\begin{array}{l}\text { Titulo da Entidade de origem. } \\
\text { Neste caso a primitiva será envi- } \\
\text { ada através de um dado Works- } \\
\text { pace porém somente a entidade } \\
\text { com o referido Titulo é que tra- } \\
\text { tará a reposta }\end{array}$ \\
\hline
\end{tabular}




\section{C.2 Entity Title Control Protocol}

A seção 3.5.1 apresentou de forma sucinta as primitivas do protocolo ETCP. A seguir são detalhados os serviços do protocolo e as primitivas envolvidas.

\section{C.2.1 ENTITY-REGISTER}

Antes de iniciar qualquer comunicação através do plano de dados, toda entidade deve solicitar o seu registro. Este registro é realizado pelo DTSA que controla o NE utilizado por essa entidade para se conectar na rede. A Figura 34 apresenta o diagrama de sequência do serviço ENTITY-REGISTER e as primitivas enviadas.

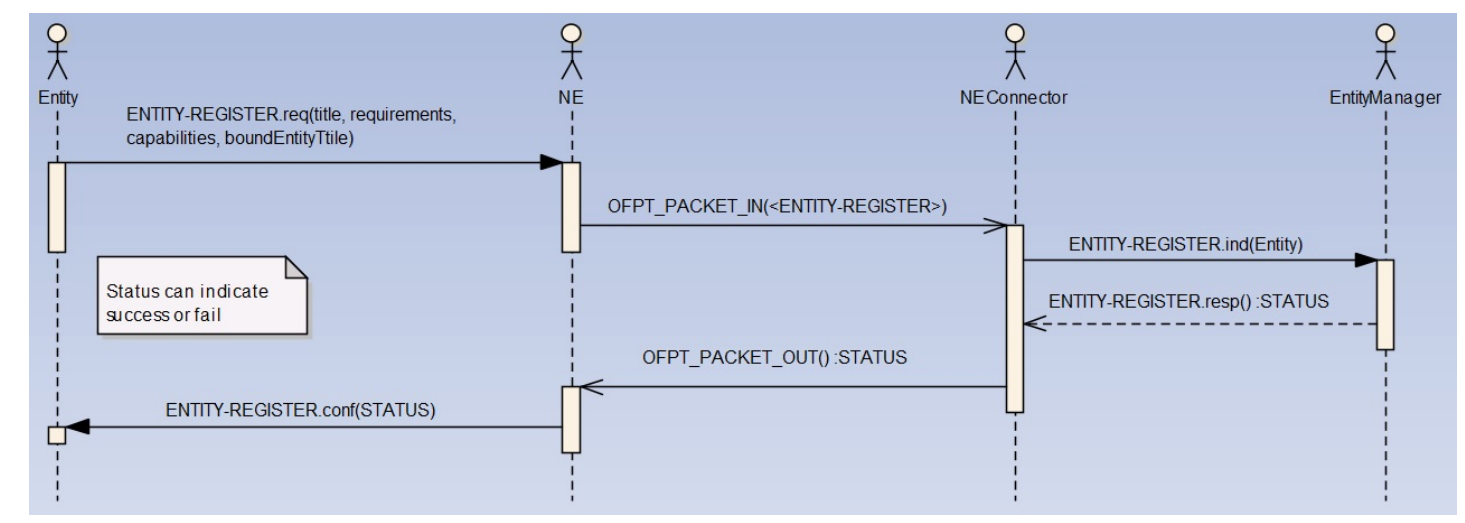

Figura 34: Diagrama de Sequência do Serviço ENTITY-REGISTER.

O diagrama de sequência apresenta os objetos envolvidos em um nível de abstração suficiente para o entendimento do serviço. Os atores envolvidos são uma Entidade (Entity), um NE que neste caso é também um switch OpenFlow e dois componentes do DTSA, o NEConnector e o EntityManager.

O NEConnector é responsável pela integração com um NE, e portanto, suporta a comunicação através do OpenFlow protocol. Ao ser notificado de um evento , o NEConnector é responsável pelo seu recebimento. Após iden- 
tificar o tipo deste evento, um novo evento é disparado a fim de ser tratado pelo EntityManager. Este módulo por sua vez é responsável pelo registro da entidade e pela manutenção de seu estado junto ao DTSA. Ao final do processo, independente de sucesso ou falha, o EntityManager produz um evento de resposta, que então é enviado à Entidade que originou o pedido.

A seguir são descritas as funções de cada primitiva envolvida neste processo.

\section{C.2.1.1 ENTITY-REGISTER.req}

Esta primitiva é responsável pelo registro de uma entidade no DTS. A fim de ser registrada uma entidade deve apresentar seu Título, suas capacidades e requisitos de comunicação. A Tabela 14 apresenta a semântica desta primitiva de serviço.

ENTITY-REGISTER.req(title, requirements, capabilities, boundEntityTtile)

Tabela 14: Semântica da Primitiva de Serviço ENTITY-REGISTER.req.

\begin{tabular}{|c|c|c|c|}
\hline NOME & TIPO DO DADO & PRESENÇA & DESCRIÇÃO \\
\hline $\begin{array}{l}\text { CONTROL } \\
\text {-PRIMITIVE }\end{array}$ & $\begin{array}{l}\text { CONTROL } \\
\text {-PRIMITIVE-TYPE }\end{array}$ & Necessário & $\begin{array}{l}\text { Campos descritos na tabela } 13 . \\
\text { Neste caso, TYPE assume o va- } \\
\text { lor ETCP-ENTITY-REGISTER = } \\
0 ;\end{array}$ \\
\hline title & TITLE-TYPE & Necessário & $\begin{array}{l}\text { Título da entidade que será re- } \\
\text { gistrada }\end{array}$ \\
\hline requirements & $\begin{array}{l}\text { LIST( } \\
\text { REQUIREMENT } \\
\text {-TYPE) }\end{array}$ & Opcional & $\begin{array}{l}\text { Lista que representa os requisi- } \\
\text { tos de comunicação que podem } \\
\text { ser associados à entidade no } \\
\text { momento de seu registro }\end{array}$ \\
\hline capabilities & $\begin{array}{l}\text { LIST( } \\
\text { REQUIREMENT } \\
\text {-TYPE) }\end{array}$ & Opcional & $\begin{array}{l}\text { Lista que representa as capaci- } \\
\text { dades de comunicação que po- } \\
\text { dem ser associadas à entidade } \\
\text { no momento de seu registro }\end{array}$ \\
\hline boundEntityTtile & TITLE-TYPE & Opcional & $\begin{array}{l}\text { Caso a entidade a ser registrada } \\
\text { esteja associada a alguma ou- } \\
\text { tra, este valor indica o seu titulo }\end{array}$ \\
\hline
\end{tabular}




\section{C.2.1.2 ENTITY-REGISTER.resp}

Esta primitiva é utilizada pelo DTSA para informar a uma entidade o resultado de uma solicitação de registro. Basicamente a resposta é uma primitiva CONTROL-RESPONSE, conforme apresentada na Figura 33(b).

\section{C.2.2 WORKSPACE-CREATE}

O Workspace, conforme definido na Seção 3.1.3, permite a comunicação entre múltiplas entidades. Antes de iniciar a troca de primitivas através do plano de dados, o Workspace deve ser criado. Uma entidade é a responsável pela criação do Workspace. A Figura 35 apresenta o diagrama de sequência do serviço WORKSPACE-CREATE e as primitivas envolvidas.

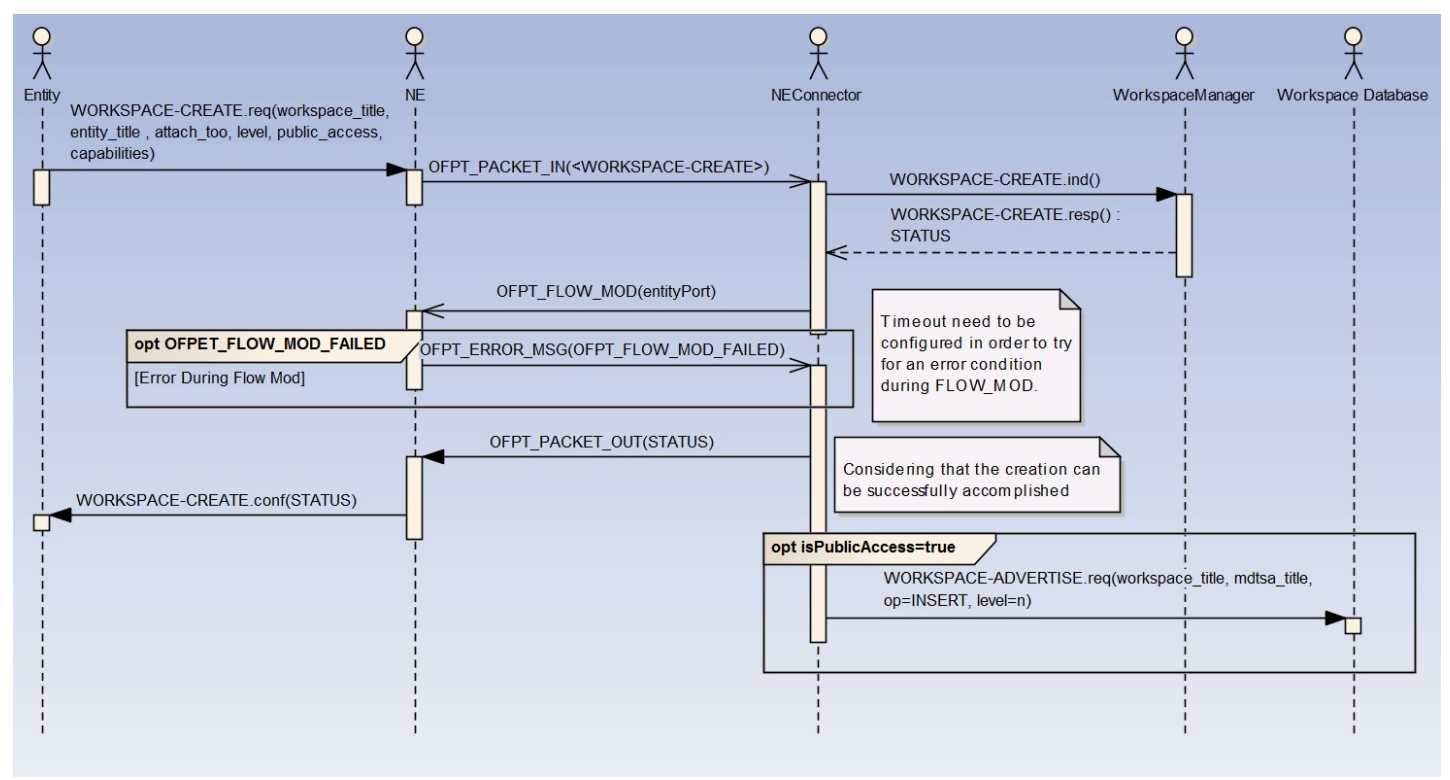

Figura 35: Diagrama de Sequência do Serviço WORKSPACE-CREATE.

Conforme mostrado no diagrama de sequência, uma entidade (Entity) envia uma requisição, representada por WORKSPACE-CREATE.req essa primitiva é então recebida pelo NE onde essa entidade está conectada. A primitiva é então encapsulada uma primitiva do OpenFlow (OFPT_PACKET_IN) que então é recebida pelo NEConnector. Este por sua vez dispara um evento que 
então é capturado pelo WorkspaceManager. Este módulo do DTSA é responsável pelo comportamento associado a cada primitiva relacionada com o Workspace. Caso o Workspace não exista então o mesmo será criado. O resultado desta operação é então devolvido para a entidade que solicitou sua criação. Caso o Workspace seja público, i.e., visível por outros DTSAs, então uma nova primitiva (WORKSPACE-ADVERTISE, veja Seção C.3.3) será enviada, neste caso pelo DTSA com destino ao MDTSA do nível (Level) correspondente. O Level é uma propriedade do DTSA, conforme definido na Seção 3.1.4. Assim que for recebido pelo MDTSA o seu título será então armazenado no Workspace Database. Por simplicidade o diagrama omite outros objetos envolvidos e apenas apresenta do Workspace Database do MDTSA dentinário da primitiva.

A seguir são descritas as funções de cada primitiva envolvida neste processo.

\section{C.2.2.1 WORKSPACE-CREATE.req}

Esta primitiva é responsável por requisitar ao DTSA a criação de um Workspace, localmente a este DTSA. Dessa forma, todas as entidades que estão registradas neste DTSA poderão posteriormente se anexar ao mesmo para comunicação. A Tabela 15 apresenta a semântica desta primitiva de serviço.

WORKSPACE-CREATE.req (workspace_title, entity_title, attach_too, level,

public_access, capabilities)

\section{C.2.2.2 WORKSPACE-CREATE.resp}

Esta primitiva é utilizada pelo DTSA para informar a uma entidade o resultado de uma solicitação de criação de um Workspace. Basicamente a res- 
Tabela 15: Semântica da Primitiva de Serviço WORKSPACE-CREATE.req.

\begin{tabular}{|c|c|c|c|}
\hline NOME & TIPO DO DADO & PRESENÇA & DESCRIÇÃO \\
\hline $\begin{array}{l}\text { CONTROL } \\
\text {-PRIMITIVE }\end{array}$ & $\begin{array}{l}\text { CONTROL } \\
\text {-PRIMITIVE-TYPE }\end{array}$ & Necessário & $\begin{array}{l}\text { Campos descritos na tabela } \\
\text { 13. Neste caso TYPE as- } \\
\text { sume o valor ETCP-WORKSPA- } \\
\text { CE-CREATE = } 2 \text {; }\end{array}$ \\
\hline workspace_title & TITLE-TYPE & Necessário & $\begin{array}{l}\text { Título do Workspace que será } \\
\text { criado }\end{array}$ \\
\hline entity_title & TITLE-TYPE & Opcional & $\begin{array}{l}\text { Título da entidade que está re- } \\
\text { quisitando a criação do Works- } \\
\text { pace }\end{array}$ \\
\hline attach_too & BOOLEAN & Opcional & $\begin{array}{l}\text { Indica se a entidade que está } \\
\text { criando o Workspace também } \\
\text { deve ser anexada ao mesmo. } \\
\text { Caso possua o valor TRUE, } \\
\text { um evento associado à pri- } \\
\text { mitiva WORKSPACE-ATTACH- } \\
\text {-IND será disparado. Caso } \\
\text { seu valor seja FALSE, então o } \\
\text { WORKSPACE não possuirá ne- } \\
\text { nhuma entidade anexada inicial- } \\
\text { mente. }\end{array}$ \\
\hline level & UNSIGNED-INT(1) & Opcional & $\begin{array}{l}\text { Caso seja informado, indica o ní- } \\
\text { vel em que o Workspace seá } \\
\text { visível. O nível equivale a dis- } \\
\text { tância até o núcleo da rede, i.e., } \\
\text { o nível zero. Seu valor deve } \\
\text { ser menor que o nível do DTSA } \\
\text { onde o Workspace será inicial- } \\
\text { mente armazenado. }\end{array}$ \\
\hline public_access & BOOLEAN & Opcional & $\begin{array}{l}\text { Indica que o Workspace a ser } \\
\text { criado será ou não visível para } \\
\text { além do nível do DTSA onde } \\
\text { será criado.Seu valor padrão é } \\
\text { FALSE indicando que o Works- } \\
\text { pace será visível apenas no } \\
\text { DTSA onde está sendo criado. }\end{array}$ \\
\hline capabilities & $\begin{array}{l}\text { LIST( } \\
\text { REQUIREMENT- } \\
\text { TYPE) }\end{array}$ & Opcional & $\begin{array}{l}\text { Representa a lista de capacida- } \\
\text { des de comunicação que será } \\
\text { associada ao workspace a ser } \\
\text { criado. }\end{array}$ \\
\hline
\end{tabular}

posta é uma primitiva CONTROL-RESPONSE, conforme apresentada na Figura 33(b). Deve ser ressaltado que caso a entidade solicite que seja anexada ao Workspace, o DTSA também enviará uma primitiva OFPT_FLOW_MOD para o switch onde a entidade está diretamente ligada. 


\section{C.2.3 WORKSPACE-ATTACH}

Após registrar-se, uma entidade pode anexar-se a algum Workspace previamente criado. Porém é necessário que os requisitos da entidade sejam satisfeitos pelas capacidades deste Workspace. A Figura 36 apresenta o diagrama de sequência do serviço WORKSPACE-ATTACH e as primitivas envolvidas.

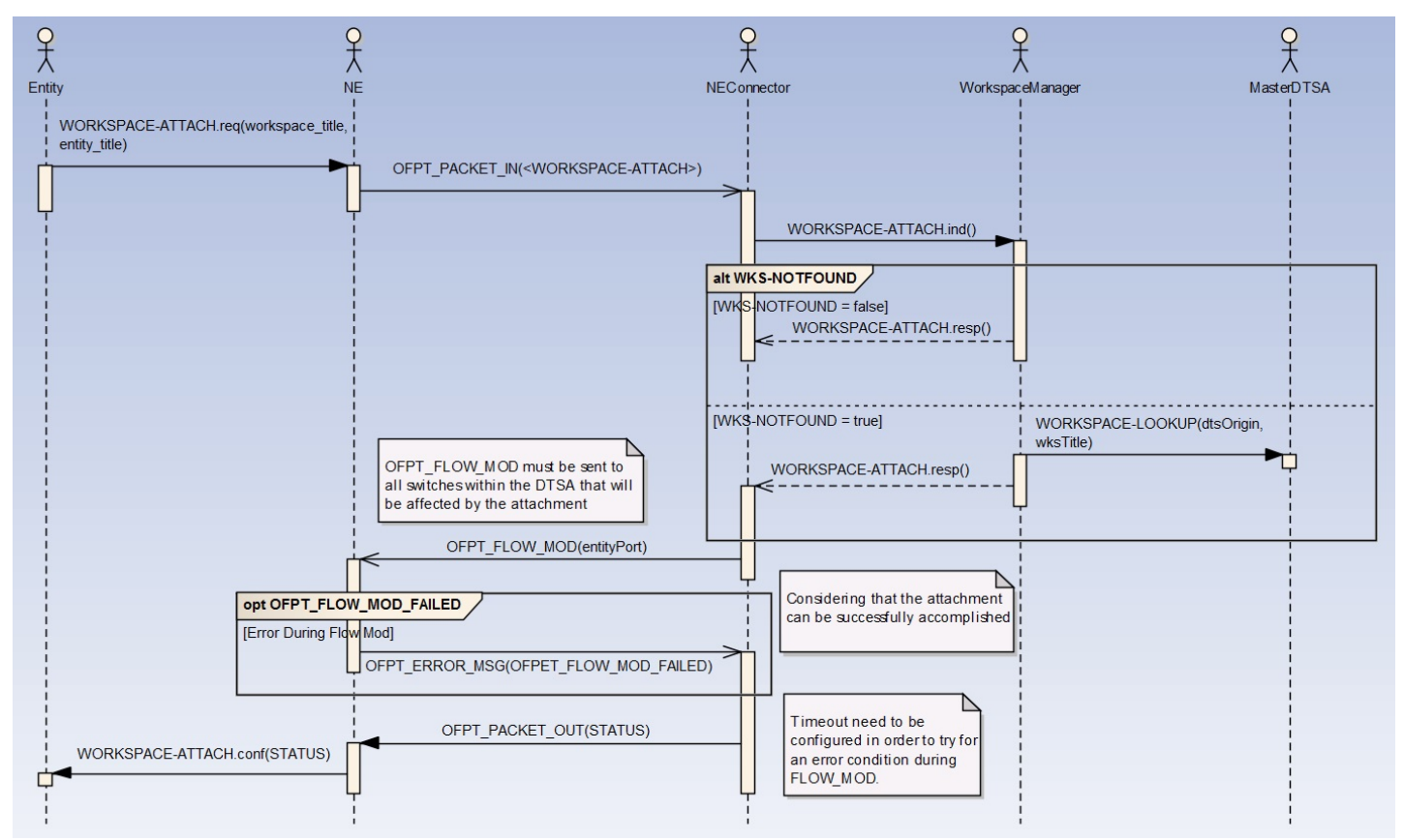

Figura 36: Diagrama de Sequência do Serviço WORKSPACE-ATTACH.

A entidade (Entity) envia uma requisição, representada por WORKSPACE-ATTACH.req esta primitiva é então recebida pelo NE onde esta entidade está conectada. A primitiva é então encapsulada em uma primitiva do OpenFlow (OFPT_PACKET_IN) que então é recebida pelo NEConnector. Este por sua vez dispara um evento que então é capturado pelo WorkspaceManager.

O DTSA possui informações sobre as entidades, os Workspaces e ainda possui um grafo com os NE que estão sob seu controle. Sendo assim, o WorkspaceManager buscará as informações do Workspace requisitado e, caso possível, anexará a entidade ao mesmo. A resposta é então enviada ao NEConnector que será responsável por interagir com os NE e modificar o 
seu comportamento, neste caso utilizando primitivas do protocolo OpenFlow (OFPT_FLOW_MOD). Ao final deste processo a primitiva WORKSPACE-ATTACH.resp será enviada a entidade informando se a mesma foi ou não anexada ao Workspace.

Caso o DTSA não conheça aquele Workspace a fim de completar a requisição será necessário uma comunicação com outros DTSAs, dentro do DTS. Para isto uma nova primitiva (WORKSPACE-LOOKUP, veja Seção C.3.4) será criada pelo DTSA e enviada com destino ao MDTSA do nível (Level) correspondente. Ao receber a primitiva o MTDSA realiza uma procura no Workspace Database e caso não encontre informações sobre aquele Workspace repetirá este processo, enviando novamente a primitiva WORKSPACE-LOOKUP para os MDTSAs de nível superior que está conectado. Este processo poderá ser repetido até chegar ao núcleo da rede (Level zero). Caso o Workspace seja encontrado a resposta será devolvida ao DTSA que originou o processo que então fará a alteração em seus NE a fim de que o Workspace chegue atá a entidade.

Por simplicidade o diagrama omite outros objetos envolvidos e apenas apresenta o MDTSA destinatário da primitiva.

\section{C.2.3.1 WORKSPACE-ATTACH.req}

Através dessa primitiva, uma entidade que deseja se comunicar, requisita ao DTSA que seja anexada a um Workspace. Após receber esta primitiva, caso seja possível anexar a entidade, o DTSA obterá todos os NE necessários e ajustará sua configuração a fim de estender este Workspace até a entidade. A Tabela 16 apresenta a semântica desta primitiva de serviço. 
Tabela 16: Semântica da Primitiva de Serviço WORKSPACE-ATTACH.req.

\begin{tabular}{llll}
\hline \hline NOME & TIPO DO DADO & PRESENÇA & DESCRIÇÃo \\
\hline \hline CONTROL & CONTROL & Necessário & $\begin{array}{l}\text { Campos descritos na tabela } \\
\text {-PRIMITIVE }\end{array}$ \\
& -PRIMITIVE-TYPE & & $\begin{array}{l}\text { 13. Neste caso TYPE assume } \\
\text { ovalor ETCP-WORKSPACE-AT- } \\
\text { TACH }=4 ;\end{array}$ \\
\hline \hline workspace_title & TITLE-TYPE & Necessário & $\begin{array}{l}\text { Título do Workspace cuja enti- } \\
\text { dade será anexada }\end{array}$ \\
\hline entity_title & TITLE-TYPE & Necessário & $\begin{array}{l}\text { Título da entidade que está re- } \\
\text { quisitando a criação do Works- } \\
\text { pace }\end{array}$ \\
\hline
\end{tabular}

\section{C.2.3.2 WORKSPACE-ATTACH.resp}

Esta primitiva é utilizada pelo DTSA para informar à entidade o resultado de sua solicitação para se anexar a um Workspace. Basicamente a resposta é uma primitiva CONTROL-RESPONSE, conforme apresentada na Figura 33(b).

\section{C.2.4 ENTITY-UNREGISTER}

Este serviço é responsável por cancelar o registro de uma entidade no DTS. Antes de efetuar o cancelamento, a entidade deve ser removida de todos os Workspaces que está anexada. Além disso, todas as outras entidades que estão a ela ligadas também devem ter o seu registro cancelado. A Figura 37 apresenta o diagrama de sequência do serviço ENTITY-REGISTER e as primitivas enviadas.

O diagrama de sequência apresenta os objetos envolvidos no serviço. Os atores envolvidos são a Entidade (Entity), um NE que neste caso é também um switch OpenFlow e dois componentes do DTSA, o NEConnector e o EntityManager. Para uma maior simplicidade o diagrama omitiu operações opcionais necessárias para remover a entidade dos Workspaces onde pode estar 


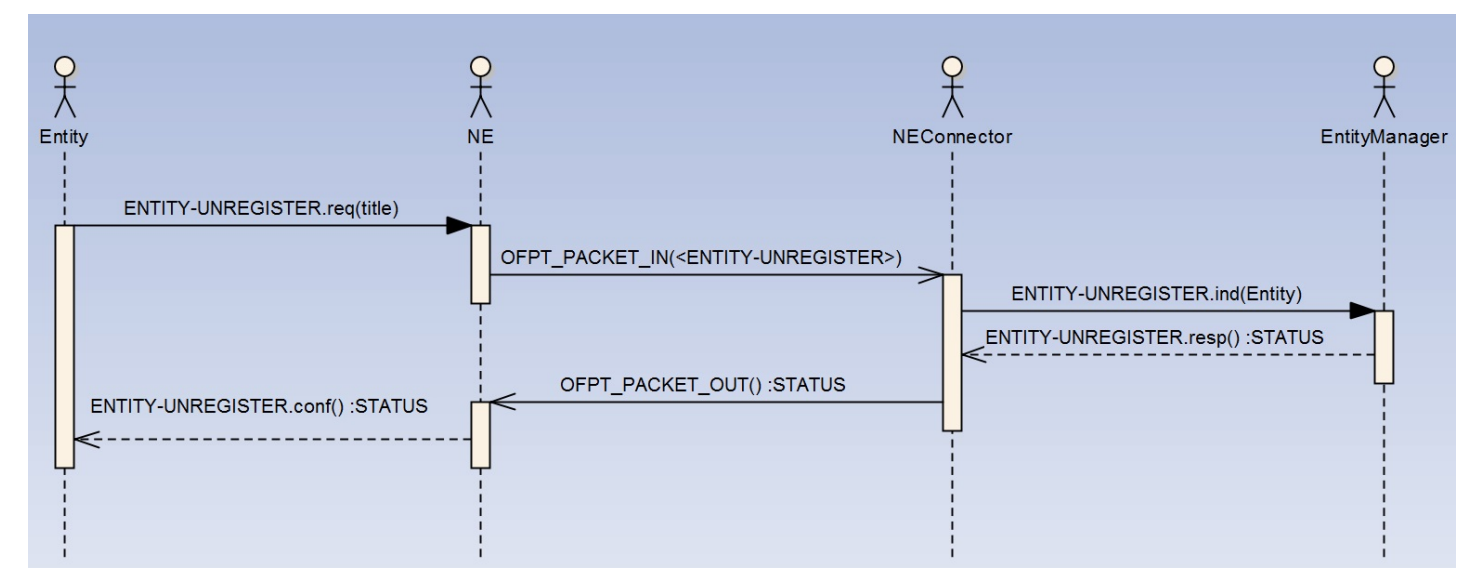

Figura 37: Diagrama de Sequência do Serviço ENTITY-UNREGISTER.

anexada.

O NEConnector é responsável pela integração com um NE e portanto suporta a comunicação através do OpenFlow protocol. Ao ser notificado de um evento, o NEConnector é responsável pelo seu recebimento. Após identificar o tipo deste evento, neste caso um novo evento é disparado a fim de ser tratado pelo EntityManager. Este módulo por sua vez é responsável pelo registro da entidade e pela manutenção de seu estado junto ao DTSA. Ao final do processo, independente de sucesso ou falha, o EntityManager produz um evento de resposta, que então é enviado à Entidade que originou o pedido.

A seguir são descritas as funções de cada primitiva envolvida neste processo.

\section{C.2.4.1 ENTITY-UNREGISTER.req}

Esta primitiva é utilizada para requisitar ao DTS o cancelamento do registro de uma entidade. Para isto é necessário informar o seu Título. A Tabela 17 apresenta a semântica desta primitiva de serviço. 
Tabela 17: Semântica da Primitiva de Serviço ENTITY-UNREGISTER.req.

\begin{tabular}{llll}
\hline \hline NOME & TIPO DO DADO & PRESENÇA & DESCRIÇÃo \\
\hline \hline CONTROL & CONTROL & Necessário & Campos descritos na tabela 13. \\
-PRIMITIVE & -PRIMITIVE-TYPE & & $\begin{array}{l}\text { Neste caso TYPE assume o } \\
\text { valor ETCP-ENTITY-UNREGIS- } \\
\end{array}$ \\
& & TER = 1; \\
\hline \hline title & TITLE-TYPE & Necessário & $\begin{array}{l}\text { Título da entidade que cujo re- } \\
\text { gistro será cancelado }\end{array}$ \\
\hline
\end{tabular}

\section{C.2.4.2 ENTITY-UNREGISTER.resp}

Esta primitiva é utilizada pelo DTSA para informar a uma entidade o resultado de uma solicitação de cancelamento do seu registro. Basicamente a resposta é uma primitiva CONTROL-RESPONSE, conforme apresentada na Figura 33(b).

\section{C.2.5 WORKSPACE-DETACH}

Caso uma entidade deseje desligar-se de um Workspace deve solicitar este serviço ao DTS. A Figura 38 apresenta o diagrama de sequência do serviço WORKSPACE-DETACH e as primitivas envolvidas.

A entidade (Entity) envia uma requisição, representada por WORKSPACEDETACH.req. Esta primitiva é então recebida pelo NE onde esta entidade está conectada. A primitiva por vez é encapsulada em uma primitiva do OpenFlow (OFPT_PACKET_IN) que então é recebida pelo NEConnector. Este por sua vez dispara um evento que então é capturado pelo WorkspaceManager.

O WorkspaceManager removerá a entidade do Workspace e atualizará as estruturas de dados envolvidas, ou seja, a lista das entidades anexadas ao Workspace e também os NE e suas respectivas portas que são utilizadas pelas entidades restantes ainda anexadas ao Workspace. A resposta é então 


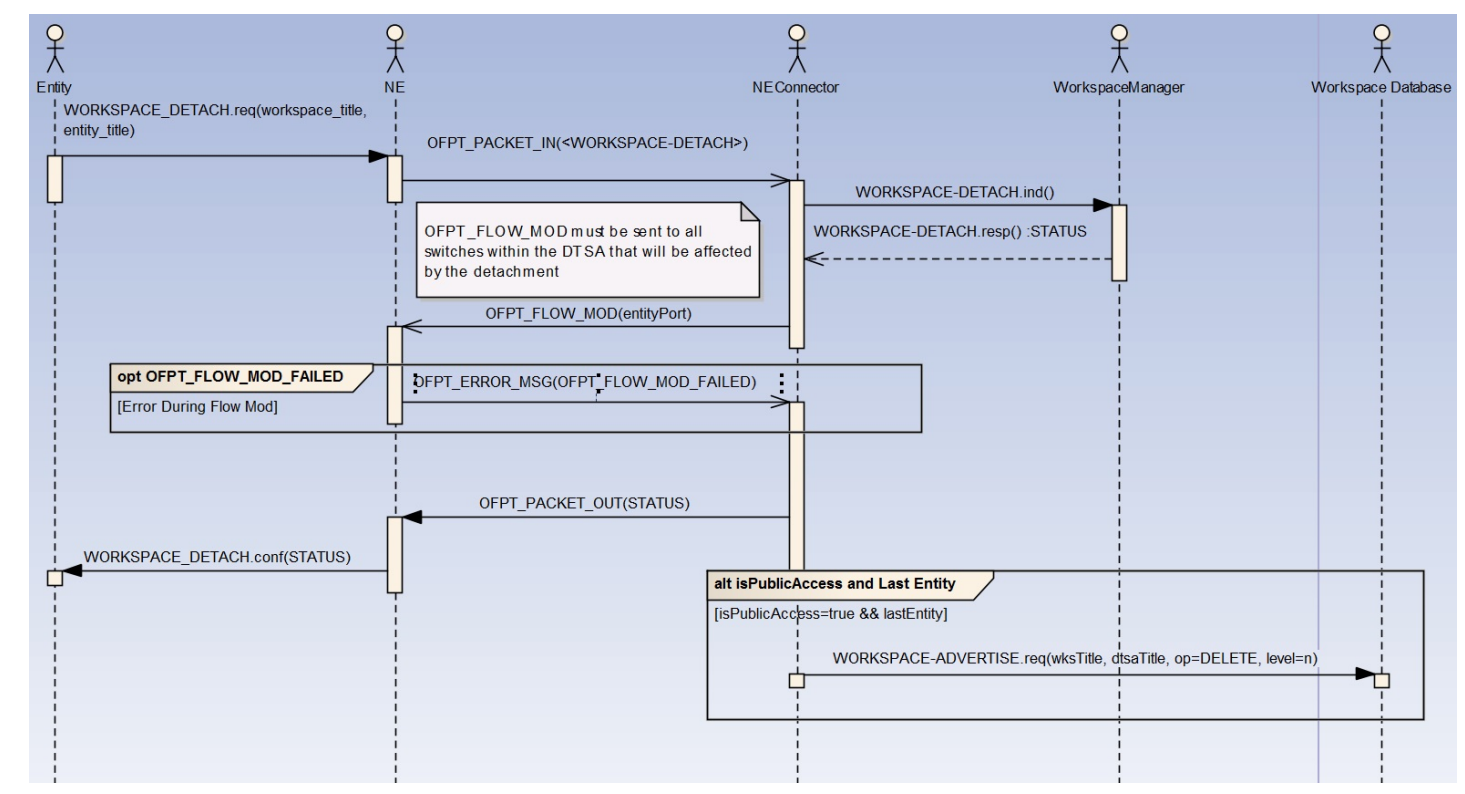

Figura 38: Diagrama de Sequência do Serviço WORKSPACE-DETACH.

enviada ao NEConnector que será responsável por interagir com os NE e, neste caso, utilizando primitivas do protocolo OpenFlow (OFPT_FLOW_MOD) os atualizará a fim de que os dados não sejam mais enviados àquela entidade. Ao final deste processo a primitiva WORKSPACE-DETACH.resp será enviada a entidade informando o resultado da requisição.

Caso o Workspace seja público e esta seja a última entidade naquele DTSA, então o DTSA deverá informar ao MDTSA que aquele Workspace não mais se encontra no seu domínio, e, portanto, deverá solicitar a atualização do Workspace Database. Para isto uma nova primitiva (WORKSPACE-ADVERTISE, veja Seção C.3.3) é criada pelo DTSA e enviada com destino ao MDTSA do nível (Level) correspondente. Ao receber a primitiva o MTDSA realizará a atualização do Workspace Database e, caso necessário, este enviará uma primitiva WORKSPACE-ADVERTISE para os MDTSAs de nível superior. Este processo poderá ser repetido até chegar ao núcleo da rede (Level zero). Por simplicidade o diagrama omite outros objetos envolvidos e apenas apresenta o Workspace Database destinatário da primitiva. 


\section{C.2.5.1 WORKSPACE-DETACH.req}

Através desta primitiva, uma entidade que deseja encerrar sua participação em um determinado Workspace, requisita esta operação ao DTS. Após receber esta primitiva, o DTSA obterá todos os NE necessários e ajustará sua configuração a fim de remover a ligação dessa entidade ao Workspace, permanecendo no mesmo apenas os NE e portas correspondentes necessários para as outras entidades ainda anexadas ao Workspace. A Tabela 18 apresenta a semântica desta primitiva de serviço.

WORKSPACE-DETACH.req (workspace_title, entity_title)

Tabela 18: Semântica da Primitiva de Serviço WORKSPACE-DETACH.req.

\begin{tabular}{|c|c|c|c|}
\hline NOME & TIPO DO DADO & PRESENÇA & DESCRIÇÃO \\
\hline $\begin{array}{l}\text { CONTROL } \\
\text {-PRIMITIVE }\end{array}$ & $\begin{array}{l}\text { CONTROL } \\
\text {-PRIMITIVE-TYPE }\end{array}$ & Necessário & $\begin{array}{l}\text { Campos descritos na tabela } 13 \text {. } \\
\text { Neste caso TYPE assume o } \\
\text { valor ETCP-WORKSPACE-DE- } \\
\text { TACH = 4; }\end{array}$ \\
\hline workspace_title & TITLE-TYPE & Necessário & $\begin{array}{l}\text { Título do Workspace onde a enti- } \\
\text { dade deseja encerrar a sua par- } \\
\text { ticipação }\end{array}$ \\
\hline entity_title & TITLE-TYPE & Necessário & $\begin{array}{l}\text { Título da entidade que está re- } \\
\text { quisitando o seu desligamento } \\
\text { do Workspace }\end{array}$ \\
\hline
\end{tabular}

\section{C.2.5.2 WORKSPACE-DETACH.resp}

Esta primitiva é utilizada pelo DTSA para informar à entidade o resultado de sua solicitação para desligar-se de Workspace. Basicamente a resposta é uma primitiva CONTROL-RESPONSE, conforme apresentada na Figura 33(b). 


\section{C.2.6 WORKSPACE-DELETE}

A entidade que inicialmente criou o Workspace pode eventualmente requisitar ao DTS que realize a remoção do mesmo. A Figura 39 apresenta o diagrama de sequência do serviço WORKSPACE-DELETE e as primitivas envolvidas.

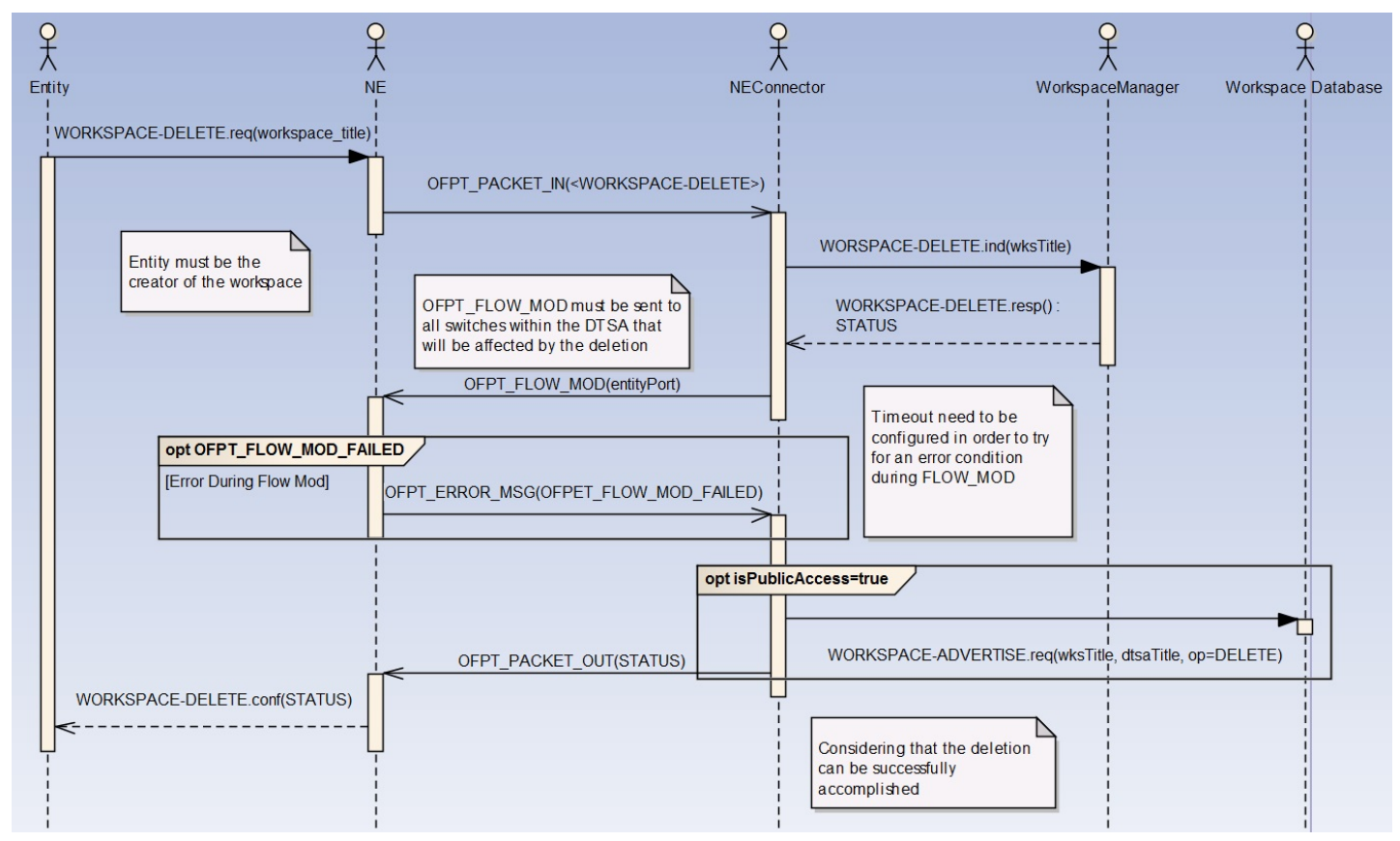

Figura 39: Diagrama de Sequência do Serviço WORKSPACE-DELETE.

A entidade (Entity) envia uma requisição, representada por WORKSPACE-DELETE.req. Esta primitiva é então recebida pelo NE onde esta entidade está conectada. A primitiva por vez é encapsulada em uma primitiva do protocolo OpenFlow (OFPT_PACKET_IN) que então é recebida pelo NEConnector. Este por sua vez dispara um evento que então é capturado pelo WorkspaceManager.

O WorkspaceManager removerá todas as entidades que estão anexadas a este Workspace e além disso retornará a lista de NE que serão afetados por esta operação no âmbito do DTSA onde inicialmente o Workspace foi criado. A resposta é então enviada ao NEConnector que será responsável por 
interagir com os NE e, neste caso, utilizando primitivas do protocolo OpenFlow (OFPT_FLOW_MOD) os atualizará a fim de que o Workspace seja efetivamente removido. Ao final deste processo a primitiva WORKSPACE-DELETE.resp será enviada a entidade informando o resultado da requisição.

Caso o Workspace seja público, então o DTSA deverá informar ao MDTSA que aquele Workspace não mais se encontra no seu domínio, e, portanto, deverá solicitar a atualização do Workspace Database. Para isto uma nova primitiva (WORKSPACE-ADVERTISE, veja Seção C.3.3) será criada pelo DTSA e enviada com destino ao MDTSA do nível (Level) correspondente. Ao receber a primitiva o MTDSA realizará a atualização do Workspace Database e, caso necessário, este enviará uma primitiva WORKSPACE-ADVERTISE para os MDTSAs de nível superior. Este processo poderá ser repetido até chegar ao núcleo da rede (Level zero). Por simplicidade o diagrama omite outros objetos envolvidos e apenas apresenta o Workspace Database destinatário da primitiva.

\section{C.2.6.1 WORKSPACE-DELETE.req}

Através desta primitiva, a entidade que criou um determinado Workspace, requisita ao DTS que realize a remoção do mesmo. Após receber essa primitiva, o DTSA obterá todos os NE necessários e ajustará sua configuração a fim de remover este Workspace. É importante destacar que nesses NEs permanecerão as informações relativas a outros Workspaces não relacionados a esta solicitação. A Tabela 19 apresenta a semântica desta primitiva de serviço. 
Tabela 19: Semântica da Primitiva de Serviço WORKSPACE-DELETE.req.

\begin{tabular}{llll}
\hline \hline NOME & TIPO DO DADO & PRESENÇA & DESCRIÇÃO \\
\hline \hline CONTROL & CONTROL & Necessário & $\begin{array}{l}\text { Campos descritos na tabela 13. } \\
\text {-PRIMITIVE }\end{array}$ \\
& -PRIMITIVE-TYPE & & $\begin{array}{l}\text { Neste caso TYPE assume o } \\
\text { valor ETCP-WORKSPACE-DE- } \\
\text { LETE = 5; }\end{array}$ \\
\hline \hline workspace_title & TITLE-TYPE & Necessário & $\begin{array}{l}\text { Título do Workspace a ser remo- } \\
\text { vido }\end{array}$ \\
\hline
\end{tabular}

\section{C.2.6.2 WORKSPACE-DELETE.resp}

Esta primitiva é utilizada pelo DTSA para informar à entidade o resultado da remoção do Workspace. Basicamente a resposta é uma primitiva CONTROL-RESPONSE, conforme apresentada na Figura 33(b).

\section{C.3 DTS Control Protocol}

A seção 3.5.2 apresentou de forma sucinta as primitivas do protocolo DTSCP. A seguir são detalhados os serviços do protocolo e as primitivas envolvidas.

\section{C.3.1 DTSA-REGISTER}

Um DTSA deve estar sob a área de influência de um ou mais MDTSA. Para isto é necessário que o DTSA registre-se junto ao MDTSA. Esta informação é uma configuração do DTSA que fica armazenada em um arquivo chamado dts-resolver.xml (ver seção 3.1.4). Este arquivo contém o Title do DTSA, o Title do Workspace de controle que é utilizado para a comunicação entre o DTSA e um MTDSA (ver seção 3.1.3.1) e finalmente os Title dos DTSAs que são vizinhos a este. Um DTSA é vizinho de outro quando existe uma ligação entre dois NEs, sendo que um está sob o controle deste DSTA e outro do 
DTSA vizinho a este.

Um DTSA deve registrar-se assim que é iniciado ou caso ocorra alguma alteração no seu arquivo de configuração (dts-resolver.xml). A Figura 40 apresenta o diagrama de sequência do serviço DTSA-REGISTER e as primitivas utilizadas.

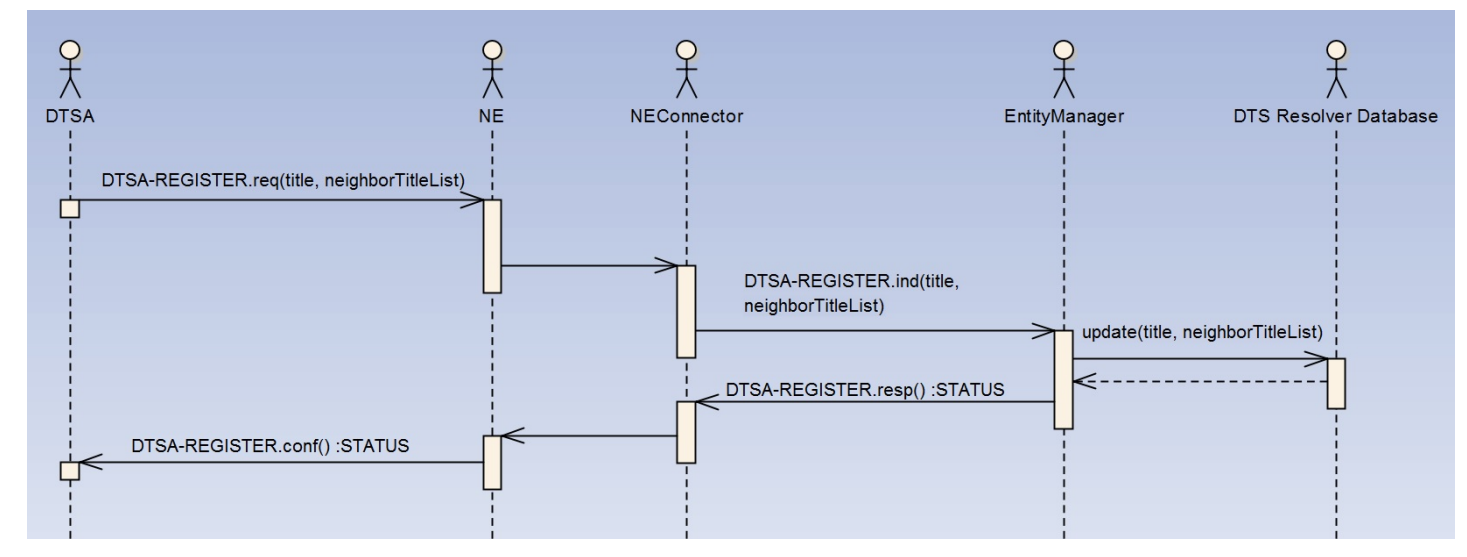

Figura 40: Diagrama de Sequência do Serviço DTSA-REGISTER.

O DTSA envia ao MDTSA cujo Title está no seu Resolver a primitiva DTSA-REGISTER.req. Esta primitiva é enviada então através do Workspace de controle, cujo Title também é parte do arquivo de configuração. Ao receber a requisição, o NEConnector deste MDTSA criará um evento que então é capturado pelo EntityManager. Em seguida o título do DTSA que está se registrando, juntamente com a lista de seus DTSAs vizinhos imediatos (neighborTitleList) serão armazenados no DTS Resolver Database. Dessa forma, para cada DTSA, o MTDSA possuirá informações sobre o Title de seus vizinhos diretos e poderá construir o grafo de DTSAs nele registrados.

Ao final do processo a resposta à requisição (DTSA-REGISTER.resp) é devolvida ao NEConnector que então trata de encaminhá-la, através do Workspace de controle, para o DTSA que originou a requisição. A seguir são descritas as funções de cada primitiva envolvida neste serviço. 


\section{C.3.1.1 DTSA-REGISTER.req}

Esta primitiva é responsável pela solicitação de registro de um DTSA junto ao MDTSA. A fim de ser registrado, um DTSA deve apresentar seu Título e uma lista com o Título de seus vizinhos, que são os DTSAs que estão diretamente ligados a este DTSA. A Tabela 20 apresenta a semântica desta primitiva de serviço.

DTSA-REGISTER.req(title, neighborTitleList)

Tabela 20: Semântica da Primitiva de Serviço DTSA-REGISTER.req.

\begin{tabular}{|c|c|c|c|}
\hline NOME & TIPO DO DADO & PRESENÇA & DESCRIÇÃO \\
\hline $\begin{array}{l}\text { CONTROL } \\
\text {-PRIMITIVE }\end{array}$ & $\begin{array}{l}\text { CONTROL } \\
\text {-PRIMITIVE-TYPE }\end{array}$ & Necessário & $\begin{array}{l}\text { Campos descritos na tabela } 13 . \\
\text { Neste caso TYPE assume o va- } \\
\text { lor DTSCP-DTSA-REGISTER = } \\
\text { 9; }\end{array}$ \\
\hline title & TITLE-TYPE & Necessário & $\begin{array}{l}\text { Título da entidade que será re- } \\
\text { gistrada }\end{array}$ \\
\hline neighborTitleList & LIST(TITLE-TYPE) & Necessário & $\begin{array}{l}\text { Lista que representa os vizinhos } \\
\text { que estão diretamente ligados } \\
\text { ao DTSA }\end{array}$ \\
\hline
\end{tabular}

\section{C.3.1.2 DTSA-REGISTER.resp}

Esta primitiva é utilizada pelo MDTSA para informar a um DTSA o resultado de uma solicitação de registro. Basicamente a resposta é uma primitiva CONTROL-RESPONSE, conforme apresentada na Figura 33(b). Deve ser ressaltado que neste caso o [SRC_TITLE] (conforme semântica apresentada na Tabela 13) deve vir preenchido com o Title do DTSA que originou a requisição. 


\section{C.3.2 DTS-MESSAGE}

Em certas situações é necessário que um DTSA necessite comunicar-se com outro DTSA. O cenário que envolve mobilidade entre DTSAs é um exemplo concreto desse requisito. Considere uma entidade, registrada no DTSA SRC $_{\text {. }}$ Ao movimentar-se, essa entidade detecta um novo PoA que neste caso está sob o domínio do DTSA ${ }_{D S T}$. Para que o handover ocorra de forma suave, sem perda de conexão, é necessário que o Workspace por onde aquela entidade está conectada seja estendido até o DTSA ${ }_{D S T}$, antes, também é necessário a entidade envolvida possa registrar-se neste DTSA.

Essas interações, conforme apresentado na seção 3.6.2, necessitam de uma troca de informações entre DTSAs. Para isto o serviço DTS-MESSAGE é utilizado. A Figura 41 apresenta o diagrama de sequência do serviço DTSMESSAGE e as primitivas envolvidas.

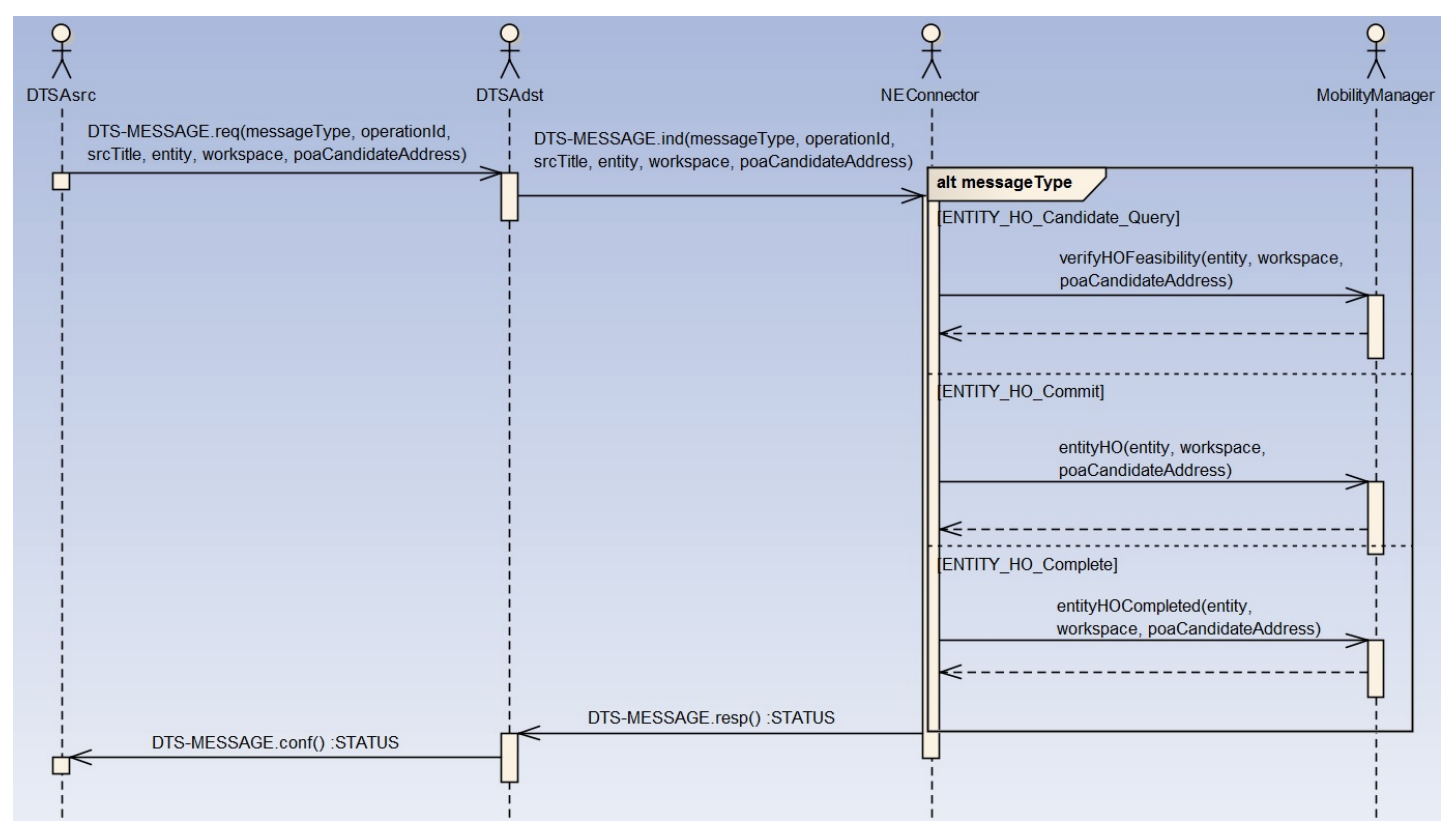

Figura 41: Diagrama de Sequência do Serviço DTS-MESSAGE.

Neste caso, o DTSA $A_{S C}$ deseja enviar uma mensagem ao DTSA $D S T$. O DTSA de origem ( $D_{T S A_{S R}}$ ) cria uma requisição, representada pela primitiva 
DTS-MESSAGE.req e a envia, através de um Workspace de controle (veja seção 3.1.3.1). Neste caso, conforme semântica apresentada na Tabela 13, o elemento CONTROL-REQUEST da primitiva (veja a Figura 33(a) deve possuir o campo [DST_TITLE] preenchido com o título do DTSA de destino ( $\left.\mathrm{DTSA}_{D S T}\right)$. Desta forma a requisição será tratada apenas pelo DTSA destinatário da primitiva. Como a primitiva pode ser utilizada para diferentes tipos de mensagens, a cada requisição será associado um tipo (messageType). Ao ser recebida pelo DTSA de destino, o NEConnector inspecionará o cabeçalho e ao identificar que aquele DTSA é o destinário, criará um evento (primitiva DTS-MESSAGE.ind) que então é capturado pelo módulo responsável pelo tratamento da mensagem. No diagrama apresentado é mostrado o caso em que o módulo é o MobilityManager, responsável pelas operações relacionadas à mobilidade no DTS. Assim que a operação é executada, uma resposta é devolvida e capturada pelo NEConnector, que finalmente envia a resposta ao DTSA que originou a requisição (primitiva WORKSPACE-ADVERTISE.resp).

A seguir são descritas as primitivas envolvidas neste serviço.

\section{C.3.2.1 DTS-MESSAGE.req}

Esta primitiva permite que o DTSA possa comunicar-se com outro. A Tabela 21 apresenta a semântica desta primitiva de serviço.

DTS-MESSAGE.req(messageType, operationId, srcTitle, entity, workspace,

poaCandidateAddress)

\section{C.3.2.2 DTS-MESSAGE.resp}

Esta primitiva é utilizada pelo DTSA ${ }_{D S T}$ para informar ao DTSA que originou a requisição $\left(D_{T S A_{S R}}\right)$ o resultado associado à mensagem enviada. Basicamente a resposta é uma primitiva CONTROL-RESPONSE, conforme apre- 
Tabela 21: Semântica da Primitiva de Serviço DTS-MESSAGE.req.

\begin{tabular}{|c|c|c|c|}
\hline NOME & TIPO DO DADO & PRESENÇA & DESCRIÇÃO \\
\hline $\begin{array}{l}\text { CONTROL } \\
\text {-PRIMITIVE }\end{array}$ & $\begin{array}{l}\text { CONTROL } \\
\text {-PRIMITIVE-TYPE }\end{array}$ & Necessário & $\begin{array}{l}\text { Campos descritos na tabela } 13 . \\
\text { Neste caso o TYPE assume o } \\
\text { valor DTSCP-DSTA-MESSAGE } \\
=10 ;\end{array}$ \\
\hline operationld & UNSIGNED-INT(2) & Necessário & $\begin{array}{l}\text { Indica um valor que pode ser as- } \\
\text { sociado à operação. Pode ser } \\
\text { utilizado pelo DTSA para criar } \\
\text { um conceito de transação entre } \\
\text { diferentes mensagens trocadas } \\
\text { entre DTSAs. }\end{array}$ \\
\hline scrTitle & TITLE-TYPE & Necessário & $\begin{array}{l}\text { Título do DTSA que está origi- } \\
\text { nando a requisição. Este título } \\
\text { do DTSA será utilizado na primi- } \\
\text { tiva de reposta a fim de que so- } \\
\text { mente o DTSA que enviou a pri- } \\
\text { mitiva receba sua resposta }\end{array}$ \\
\hline entity & ENTITY & Opcional & $\begin{array}{l}\text { Indica as informações referen- } \\
\text { tes a uma entidade. Neste } \\
\text { caso equivale aos dados associ- } \\
\text { ados à primitiva ENTITY-REGIS- } \\
\text { TER.req (descritos na Tabela } \\
\text { 14) }\end{array}$ \\
\hline workspace & WORKSPACE & Opcional & $\begin{array}{l}\text { Indica as informações referente } \\
\text { a um Workspace. Neste caso } \\
\text { equivale aos dados associados } \\
\text { à primitiva WORKSPACE-CRE- } \\
\text { ATE.req (descritos na Tabela } \\
\text { 15) }\end{array}$ \\
\hline $\begin{array}{l}\text { poaCandidate } \\
\text { Address }\end{array}$ & Opcional & TITLE-TYPE & $\begin{array}{l}\text { Indica o título de PoA. Neste } \\
\text { caso o título equivale a uma } \\
\text { identificação de um link associ- } \\
\text { ado a um NE. }\end{array}$ \\
\hline
\end{tabular}

sentada na Figura 33(b). Deve ser ressaltado que neste caso o [SRC_TITLE] (conforme semântica apresentada na Tabela 13) deve vir preenchido com o Title do DTSA que originou a requisição.

\section{C.3.3 WORKSPACE-ADVERTISE}

Quando um Workspace é criado o mesmo está presente apenas no âmbito do DTSA que o contém. Desta forma, para que fique visível e acessível por 
outros DTSAs é necessário publicá-lo. A publicação consiste em atualizar o Workspace Database e, portanto, esta informação é mantida pelo MDTSA. A publicação ocorre inicialmente no mesmo Level do DTSA que criou o Workspace e caso o Level informado seja menor que o associado ao DTSA que originou o processo é propagado em direção ao núcleo da rede (Level zero) e neste caso, o Workspace possuirá uma visibilidade por todo o DTS. A Figura 42 apresenta o diagrama de sequência do serviço WORKSPACE-ADVERTISE e as primitivas envolvidas.

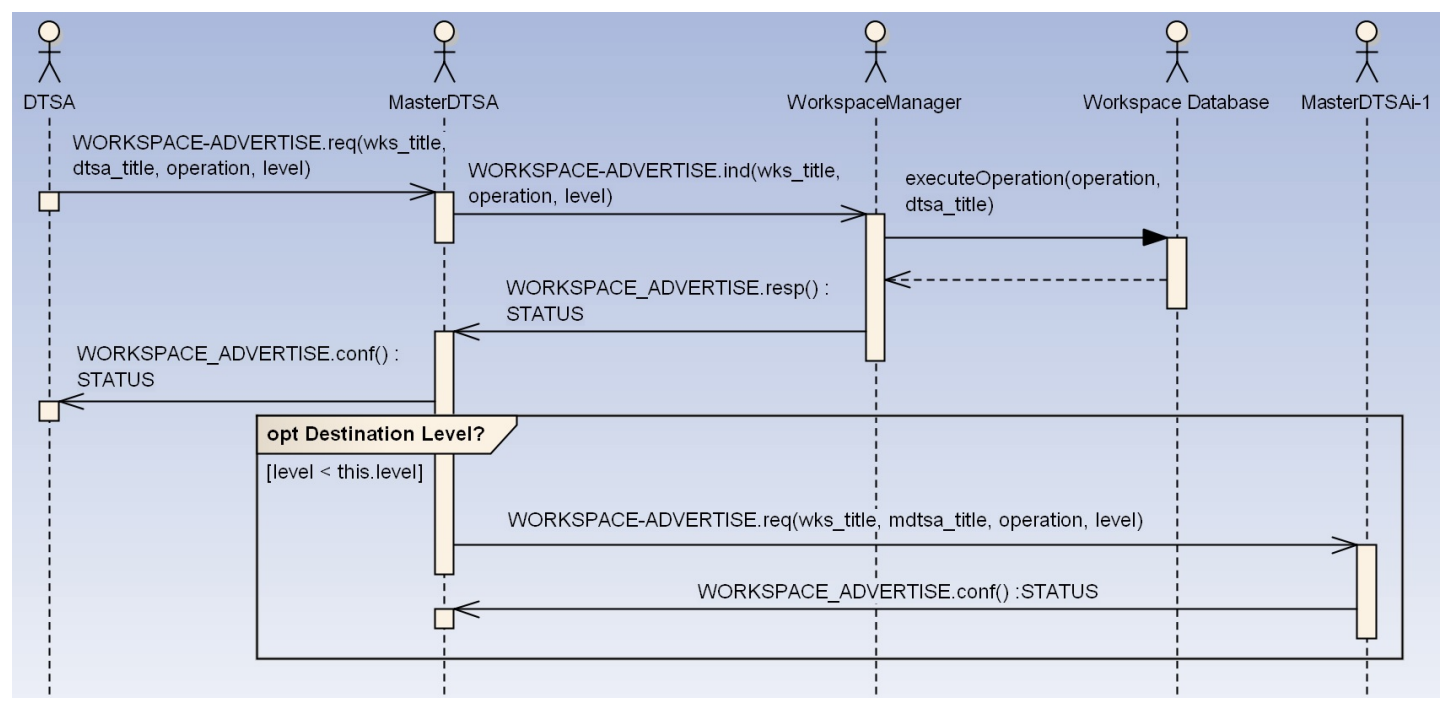

Figura 42: Diagrama de Sequência do Serviço WORKSPACE-ADVERTISE.

Caso um Workspace seja criado ou removido de um DTSA esta informação deverá ser enviada ao MDTSA onde aquele DSTA está registrado. Para isto o DTSA envia uma requisição, representada pela primitiva WORKSPACE-ADVERTISE.req. Ao enviar a requisição o DTSA indica até qual Level será necessário publicar as informações do Workspace. O valor operation indica se é necessário atualizar ou remover as informações acerca de um dado Workspace (wks_tile).

Assim que o MDTSA recebe esta requisição (primitiva WORKSPACE-ADVERTISE.ind), o NEConnector criará um evento que então é capturado pelo 
WorkspaceManager. A operação (operation) indicada é então realizada sobre Workspace Database. Esta operação poderá inserir, ou remover, o Title do DTSA para o referido Workspace. Assim que a operação é finalizada seu resultado é enviado ao DTSA que originou a requisição (primitiva WORKSPACE-ADVERTISE.resp).

Caso o parâmetro Level seja menor que o Level do MDTSA corrente, uma nova primitiva (WORKSPACE-ADVERTISE.req) será criada pelo MDTSA a fim de publicar no MDTSA do próximo nível $\left(\mathrm{MDTSA}_{i-1}\right)$ informações sobre este Worspace. Neste caso será atualizado no Workspace Database o Title do MDTSA que originou a requisição. Este processo pode repetir-se a fim de propagar a informação até o núcleo da rede, i.e., o nível zero. A Figura 42 apresenta um passo genérico deste processo envolvendo o $\mathrm{MDTSA}_{i}$ e $\circ$ MDTSA $_{i-1}$.

A seguir são descritas as primitivas envolvidas neste serviço.

\section{C.3.3.1 WORKSPACE-ADVERTISE.req}

Através desta primitiva, um DTSA publica as informações de um Workspace no MDTSA que está registrado, ou, então, um MDTSA publica tal informação no MDTSA que está no nível seguinte. A Tabela 22 apresenta a semântica desta primitiva de serviço.

WORKSPACE-ADVERTISE.req(wks_title, dtsa_title, operation, level)

\section{C.3.3.2 WORKSPACE-ADVERTISE.resp}

Esta primitiva é utilizada pelo MDTSA para informar a um DTSA o resultado de uma solicitação de publicação do Workspace. Basicamente a resposta é uma primitiva CONTROL-RESPONSE, conforme apresentada na $\mathrm{Fi}-$ 
Tabela 22: Semântica da Primitiva de Serviço WORKSPACE-ADVERTISE.req.

\begin{tabular}{|c|c|c|c|}
\hline NOME & TIPO DO DADO & PRESENÇA & DESCRIÇÃO \\
\hline $\begin{array}{l}\text { CONTROL } \\
\text {-PRIMITIVE }\end{array}$ & $\begin{array}{l}\text { CONTROL } \\
\text {-PRIMITIVE-TYPE }\end{array}$ & Necessário & $\begin{array}{l}\text { Campos descritos na tabela } 13 \text {. } \\
\text { Neste caso O TYPE assume } \\
\text { o valor DTSCP-WORKSPACE- } \\
\text {-ADVERTISE = 8; }\end{array}$ \\
\hline wks_title & TITLE-TYPE & Necessário & $\begin{array}{l}\text { Título do Workspace que será } \\
\text { publicado }\end{array}$ \\
\hline dtsa_title & TITLE-TYPE & Necessário & $\begin{array}{l}\text { Título do DTSA ou MDTSA que } \\
\text { está originando a requisição. } \\
\text { Esta informação será atualizada } \\
\text { no Workspace Database do des- } \\
\text { tinatário desta requisição }\end{array}$ \\
\hline operation & UNSIGNED-INT(1) & Necessário & $\begin{array}{l}\text { Indica a operação a ser reali- } \\
\text { zada no Workspace Database. }\end{array}$ \\
\hline level & UNSIGNED-INT(1) & Necessário & $\begin{array}{l}\text { Indica o nível até onde o Works- } \\
\text { pace deverá ser publicado visí- } \\
\text { vel. O nível equivale a distância } \\
\text { até o núcleo da rede, i.e., o nível } \\
\text { zero. Seu valor pode ser menor } \\
\text { que o nível do DTSA onde a re- } \\
\text { quisição é inicialmente criada. }\end{array}$ \\
\hline
\end{tabular}

gura 33(b). Deve ser ressaltado que neste caso o [SRC_TITLE] (conforme semântica apresentada na Tabela 13) deve vir preenchido com o Title do DTSA ou MDTSA que originou a requisição.

\section{C.3.4 WORKSPACE-LOOKUP}

Na situação em que entidade deseje anexar-se a um Workspace e o DTSA onde esta entidade está registrada não possuir informações a respeito deste Workspace ele utilizará o serviço WORKSPACE-LOOKUP. A Figura 43 apresenta o diagrama de sequência do serviço WORKSPACE-LOOKUP e as primitivas envolvidas.

Inicialmente o DTSA envia uma requisição ao MDTSA em que está registrado. Ao receber a requ isição, representada pela primitiva WORKSPA- 


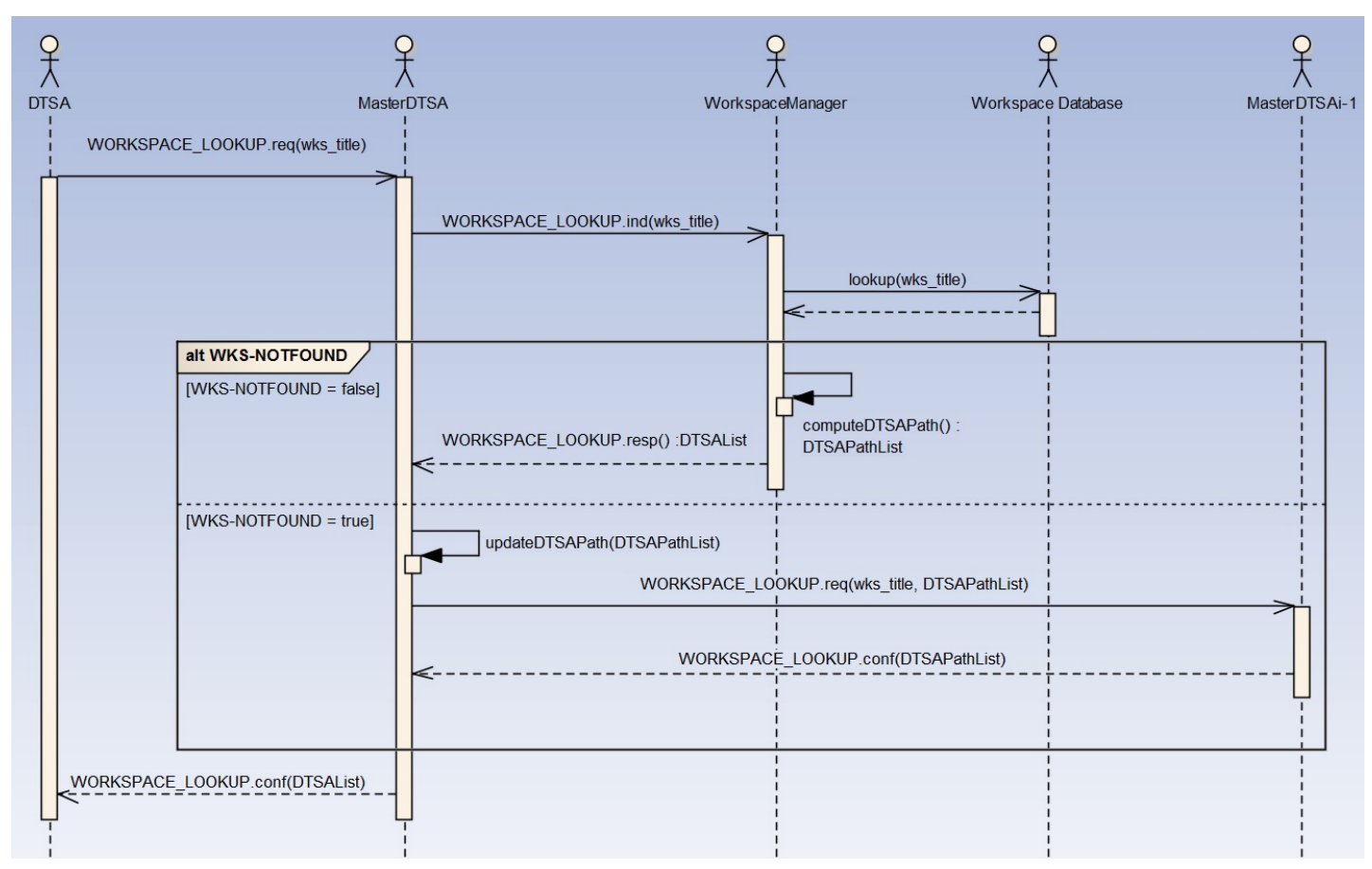

Figura 43: Diagrama de Sequência do Serviço WORKSPACE-LOOKUP.

CE-LOOKUP.req, o NEConnector deste MDTSA criará um evento (primitiva WORKSPACE-LOOKUP.ind) que então é capturado pelo WorkspaceManager. O MDTSA que possui dos DTSAs a ele registrados, realizará uma procura no Workspace Database. Caso encontre o Workspace então o mesmo realizará um cálculo a fim de encontrar o caminho de menor custo. Este caminho contém uma lista de DTSAs (representado no diagrama por DTSAList) que permite ao DTSA que originou a requisição chegar até o Workpsace. Dessa forma o Workspace deverá ser estendido e ao final do processo ele estará presente em todos estes DTSAs presentes nesta lista. Ao final do processo a resposta à requisição (WORKSPACE-LOOKUP.resp) é devolvida ao NEConnector que então trata de encaminhar a resposta do DTSA que originou a requisição.

Caso este MDTSA não possua informações sobre o referido Workspace então o processo será reiniciado. Neste caso o MDTSA, que está no nível (Level i) enviará a consulta ao MDTSA que esta em sua lista de Resolvers. Neste caso um MDTSA do nível (Level i-1). A medida que esta consulta é 
propagada em direção ao núcleo da rede, i.e., o nível zero, cada MDTSA que envia a requisição inclui do seu Title na lista de DTSAs ( DTSAPathList). Na pior das hipóteses esta requisição chegará ao núcleo da rede e, neste caso, se o Workspace não for encontrado a busca falhará. A Figura 430 diagrama de sequência apresenta um passo genérico deste processo envolvendo o $\mathrm{MDTSA}_{i}$ e o MDTSA $\mathrm{M}_{i-1}$.

\section{C.3.4.1 WORKSPACE-LOOKUP.req}

Através desta primitiva, a entidade criou um determinado Workspace, requisita ao DTS que realize a remoção do mesmo. Após receber esta primitiva, o DTSA obterá todos os NE necessários e ajustará sua configuração a fim de remover este Workspace. É importante destacar que nestes NE permanecerão as informações relativas a outros Workspaces não relacionados a esta solicitação. A Tabela 23 apresenta a semântica desta primitiva de serviço.

WORKSPACE-LOOKUP.req (workspace_title, DTSAPathList)

Tabela 23: Semântica da Primitiva de Serviço WORKSPACE-LOOKUP.req.

\begin{tabular}{|c|c|c|c|}
\hline NOME & TIPO DO DADO & PRESENÇA & DESCRIÇÃO \\
\hline $\begin{array}{l}\text { CONTROL } \\
\text {-PRIMITIVE }\end{array}$ & $\begin{array}{l}\text { CONTROL } \\
\text {-PRIMITIVE-TYPE }\end{array}$ & Necessário & $\begin{array}{l}\text { Campos descritos na tabela } 13 . \\
\text { Neste caso o TYPE assume } \\
\text { o valor DTSCP-WORKSPACE- } \\
\text { LOOKUP = 7; }\end{array}$ \\
\hline workspace_title & TITLE-TYPE & Necessário & $\begin{array}{l}\text { Título do Workspace que será } \\
\text { procurado }\end{array}$ \\
\hline DTSAPathList & $\begin{array}{l}\text { LIST(DTSA-PATH- } \\
\text { TYPE) }\end{array}$ & Opcional & $\begin{array}{l}\text { Lista que representa um cami- } \\
\text { nho entre DTSAS. Cada ele- } \\
\text { mento desta lista contém o Title } \\
\text { do DTSA e o custo associado ao } \\
\text { caminho entre os DTSAs } i-1 \text { e } \\
i\end{array}$ \\
\hline
\end{tabular}




\section{C.3.4.2 WORKSPACE-LOOKUP.resp}

Esta primitiva é utilizada pelo MDTSA para informar ao DTSA (ou MDTSA) que originou a requisição o resultado do processo de busca por um Workspace. A Tabela 24 apresenta a semântica desta primitiva de serviço.

WORKSPACE-LOOKUP. resp (DTSAPathList)

Tabela 24: Semântica da Primitiva de Serviço WORKSPACE-LOOKUP.resp.

\begin{tabular}{llll}
\hline \hline NOME & TIPO DO DADO & PRESENÇA & DESCRIÇÃO \\
\hline \hline CONTROL & CONTROL & Necessário & Campos descritos na tabela 13. \\
-RESPONSE & -RESPONSE & & \\
& -TYPE & & \\
\hline \hline DTSAPathList & LIST(DTSA-PATH- & Necessário & Lista de tuplas que representa \\
& TYPE) & & $\begin{array}{l}\text { um caminho entre DTSAS. } \\
\text { Cada elemento desta lista con- } \\
\end{array}$ \\
& & tém o Title do DTSA e o custo \\
& & associado ao mesmo \\
\hline
\end{tabular}

